\title{
Cross-borehole DC resistivity tomography of sea ice: \\ temporal and spatial variations in the anisotropic microstructure
}

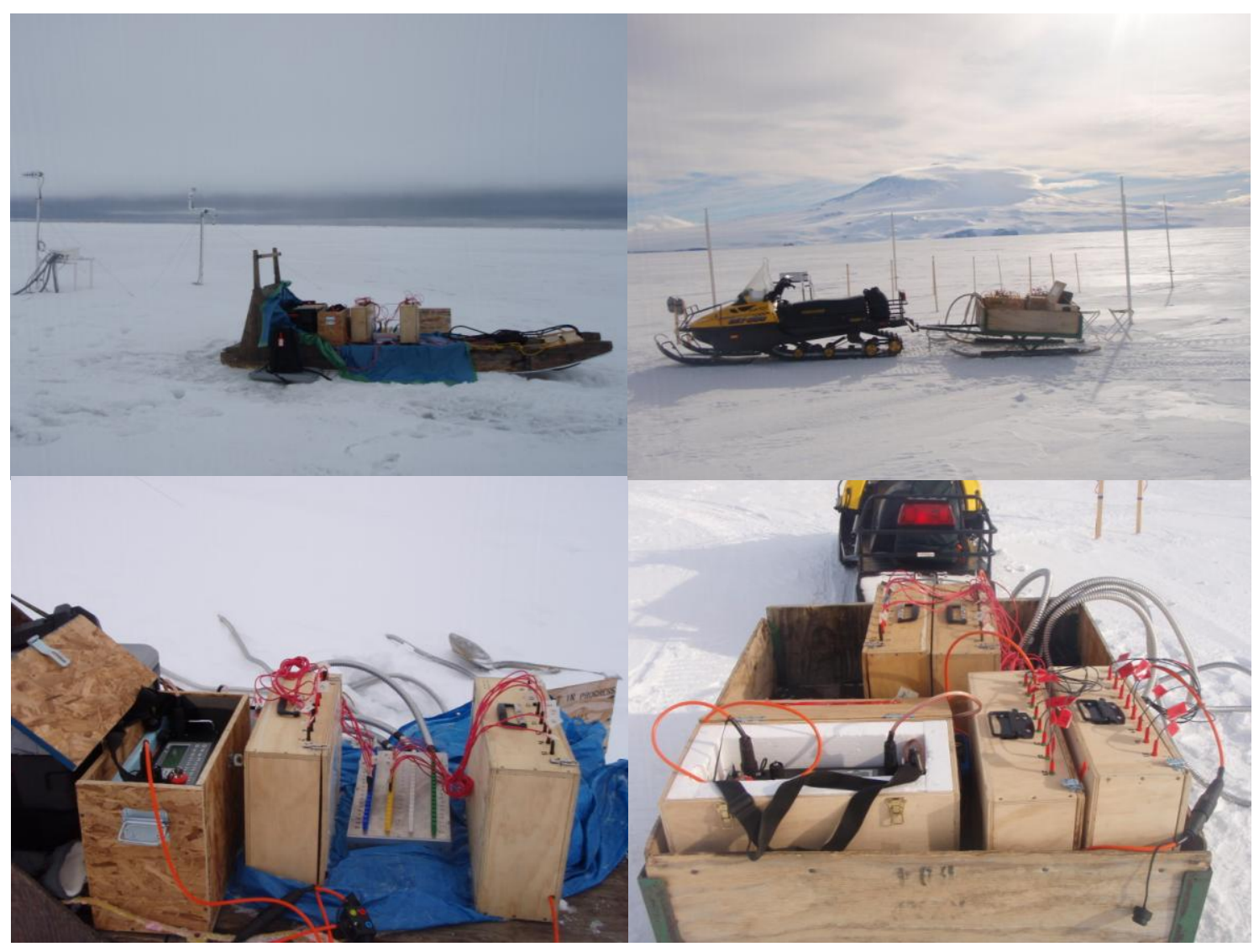

By

Keleigh Ann Jones

A thesis submitted to The School of Chemical and Physical Sciences, Victoria University of Wellington in fulfilment of the requirements for the degree of Doctor of Philosophy in Physics

Victoria University of Wellington 2011 



\section{Abstract}

As an inhomogeneous mixture of pure ice, brine, air and solid salts the physical properties of sea ice depend on its highly temperature-dependent microstructure. Understanding the microstructure and the way it responds to variations in temperature and salinity is crucial in developing an improved understanding of the interaction between sea ice and the environment. However, measurements monitoring the microstructure of sea ice are difficult to obtain without disturbing its natural state.

The brine fraction of sea ice is orders of magnitude more conductive than the solid ice, thus direct current resistivity techniques should yield information on sea ice microstructure. Due to the preferential vertical alignment of brine inclusions, the bulk resistivity of first-year sea ice is anisotropic, complicating interpretation of surface resistivity soundings. However, it can be shown that in a bounded anisotropic medium the resistivity structure may be resolved through in situ cross-borehole measurements.

Measurement between borehole pairs, each containing one current and one potential electrode, allows the determination of the horizontal component of the anisotropic bulk resistivity $\left(\rho_{H}\right)$. Using three to four electrodes positioned at approximately the same depth in separate boreholes, provides an under-estimation of the geometric mean resistivity $\left(\rho_{m}\right)$, and numerical modelling is required to retrieve an estimate of the true $\rho_{m}$. Combining these resistivities allows calculation of the vertical component of the bulk resistivity $\left(\rho_{V}\right)$.

This thesis looks at results from measurements made in first year sea ice in April - June 2008 off Barrow, Alaska and in November 2009 off Ross Island, Antarctica. At Barrow, relatively quiescent conditions typically lead to a predominance of columnar ice, while more turbulent conditions and underwater ice formation in McMurdo Sound tend to produce a larger component of frazil or platelet ice.

Interpretation of the resistivity measurements, aided by temperature and salinity data, shows that this measurement technique can be used to observe evolution of the ice structure, and distinguish different ice types. Basic two phase structures provide a simple picture of the brine microstructure and how it changes with depth and time. These models indicate the need for vertical connectivity of the brine inclusions even in cool ice, and that $\rho_{H}$ seems to be mostly due to connections along grain boundaries. 



\section{Acknowledgements}

I would like to acknowledge and express my appreciation to all those who, in some way, supported me during the course of this study.

Firstly a big thanks to my primary supervisor Dr. Malcolm Ingham (Victoria University of Wellington (VUW)). He suggested the topic and organised the field work, not to mention providing me with the opportunity to travel to the Arctic and Antarctica. Time was made in an often busy schedule to provide advice and direction throughout the project, and always with good humour. Additionally, support and expertise were always available from my secondary supervisor Emeritus Professor Joe Trodahl (VUW) and other members of the VUW sea ice group.

During this research I received financial support from a VUW Doctoral Scholarship/Assistantship. Travel to Alaska was funded by a S. T. Lee young researcher travel award which allows for Graduate student exchange between; the Antarctic Research Centre, VUW and the International Arctic Research Center, University of Alaska Fairbanks (UAF).

The Arctic field measurements were made with, financial support obtained through NSF Office of Polar Programs, grants ARC-0620124 and 0934683. Logistic support was provided by the Barrow Arctic Science Consortium. Access to data and field assistance were supplied by Professor Hajo Eicken, Dr Daniel Pringle, and the floating ice group at UAF. Hajo also provided invaluable feedback throughout my research. Daniel and wife Lisa kindly opened their home to me. Insight, into what was a new field for me, was gained from a sea ice field course, attended by international experts and students.

Antarctic field measurements were made as part of science event K131, collaboration between the University of Otago (UoO), VUW, National Institute of Water and Atmospheric Research, and Industrial Research Ltd. (IRL), coordinated by Dr Tim Haskell (IRL). Logistics support was provided by Antarctic New Zealand. Alex Gough and Andy Mahoney (UoO) installed the electrode strings. Dr Robin Dykstra and the NMR group (VUW), and Alex (UoO) kindly supplied me with access to their data.

Last but not least I would like to thank my friends and family for their continual support throughout the whole process. 



\section{Contents}

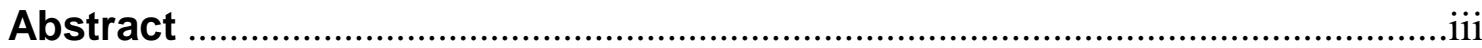

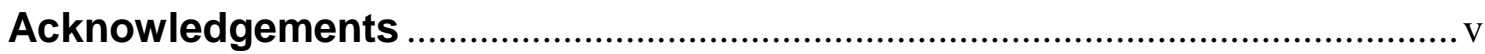

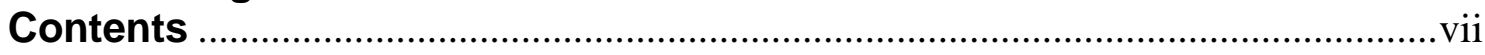

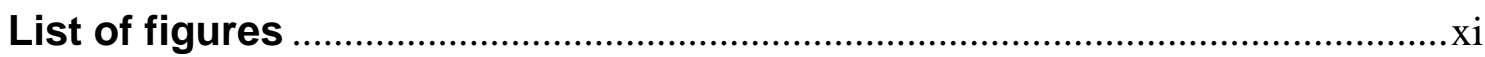

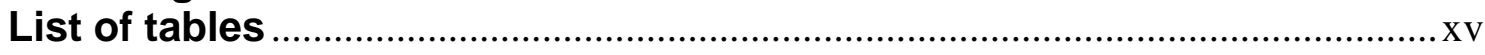

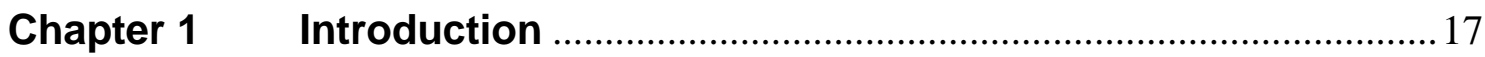

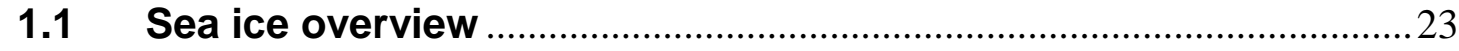

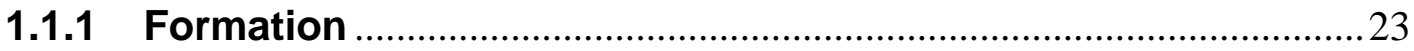

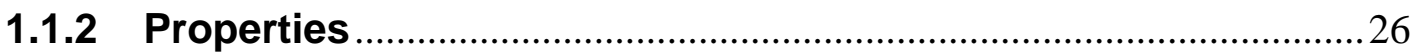

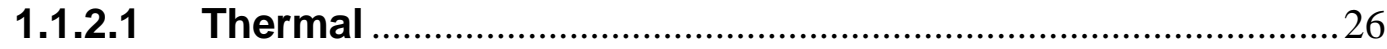

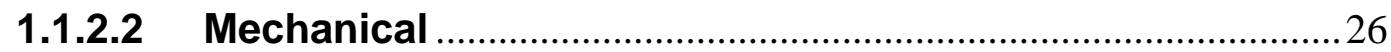

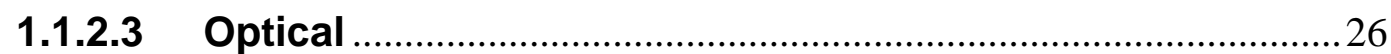

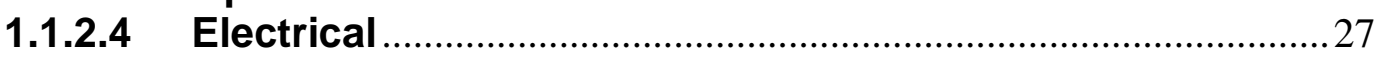

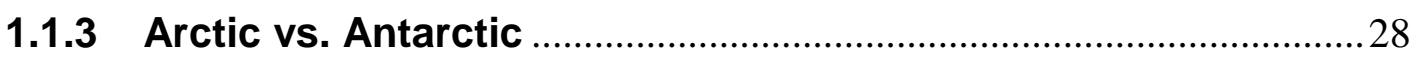

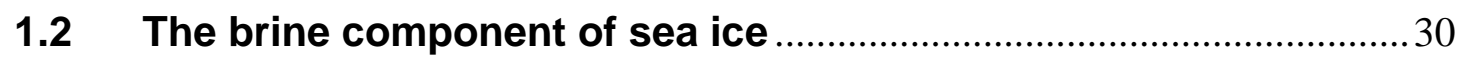

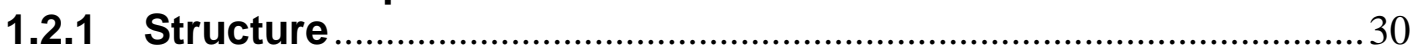

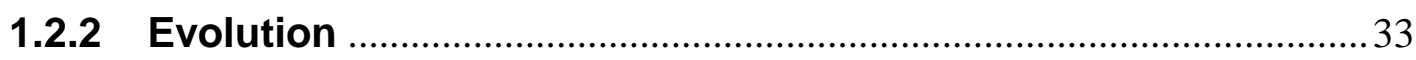

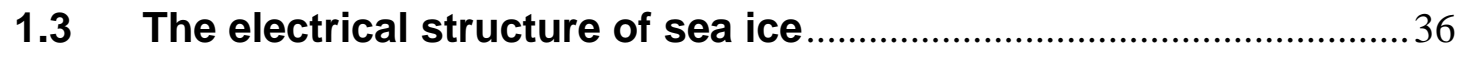

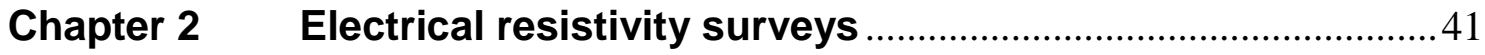

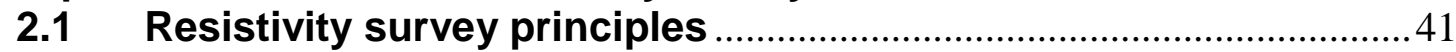

2.1.1 Resistivity of an isotropic medium............................................... 41

2.1.2 Resistivity of an anisotropic medium ......................................... 43

2.1.3 Surface electrical resistivity surveys............................................46

2.1.3.1 Measurements on a semi-infinite uniform anisotropic medium ....................................................................................... 47

2.1.4 Borehole resistivity measurements ............................................ 48

2.1.4.1 Measurements in a infinite uniform anisotropic medium

2.2 Cross-borehole resistivity measurements in a infinite

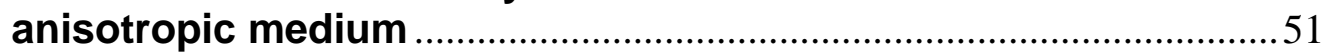

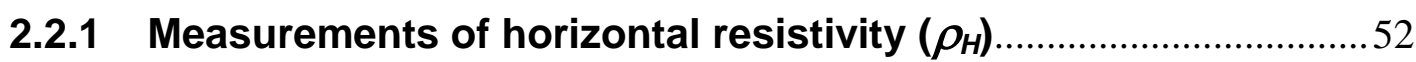

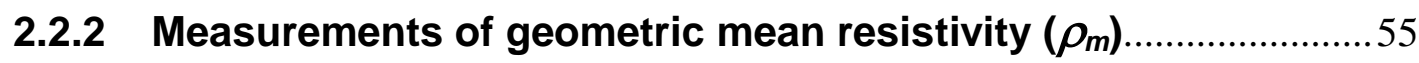

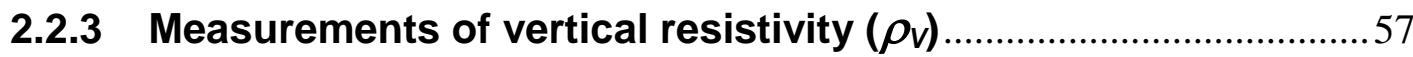

2.3 Cross-borehole resistivity measurements in a bounded anisotropic medium

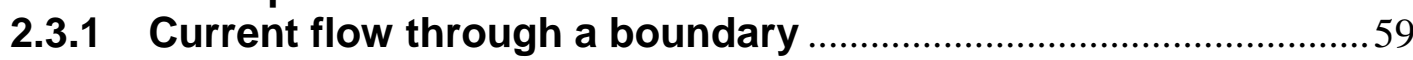

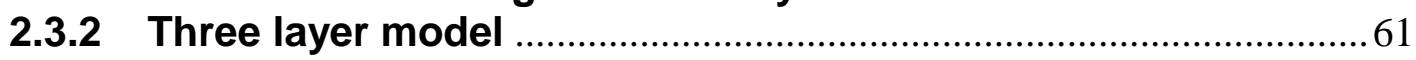

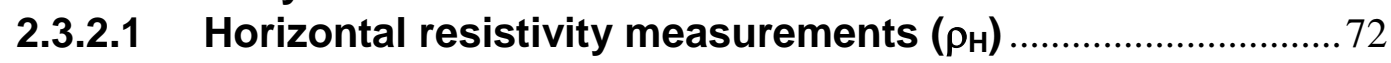

2.3.2.2 Geometric mean resistivity measurements $\left(\rho_{\mathrm{m}}\right) \ldots \ldots \ldots \ldots \ldots \ldots . . . . . .77$

2.3.2.3 Surface resistivity measurements ...................................... 81

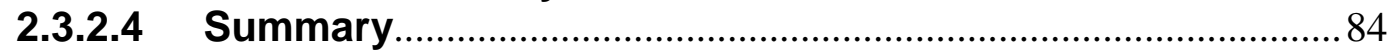

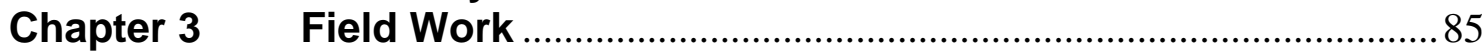

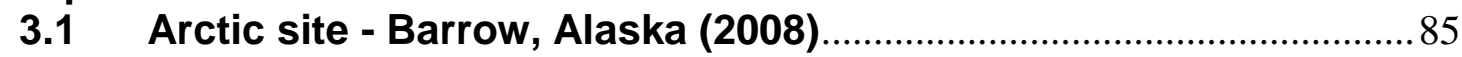

3.2 Antarctic site - McMurdo sound, Ross Dependency (2009) .............87

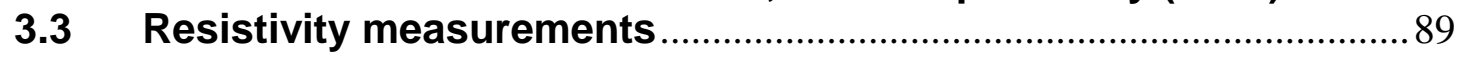

3.3.1 Horizontal resistivity measurements ........................................... 91

3.3.2 Geometric mean resistivity measurements ............................... 94

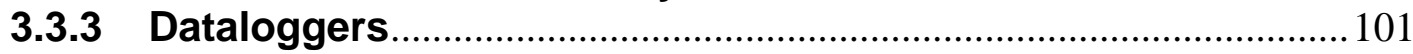


3.4 Ice thickness and snow depth measurements ................................. 104

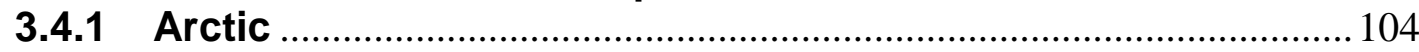

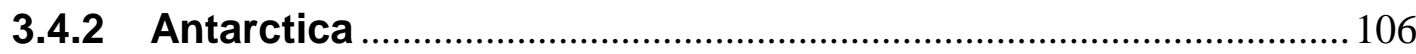

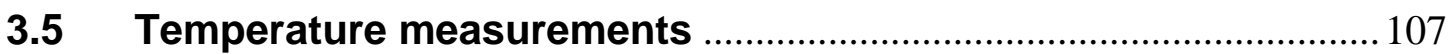

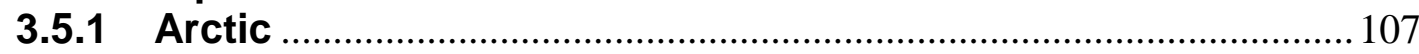

3.5.2 Antarctica ................................................................................. 110

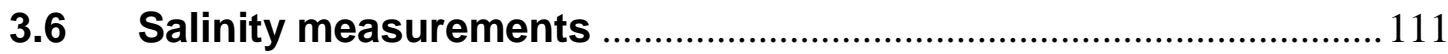

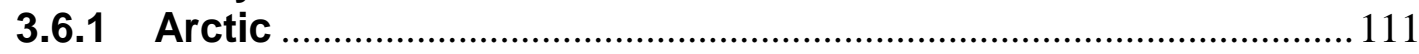

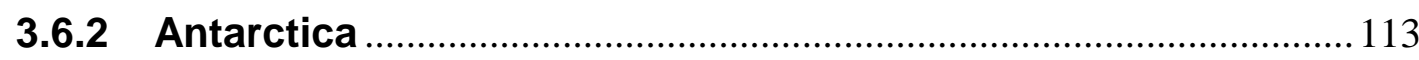

3.7 Brine volume fraction calculations................................................... 114

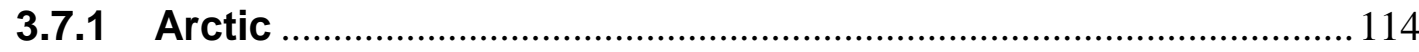

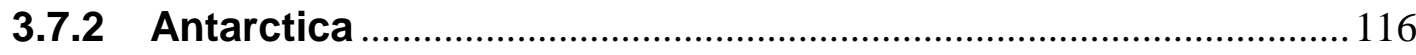

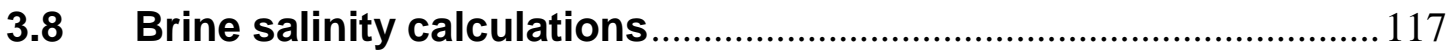

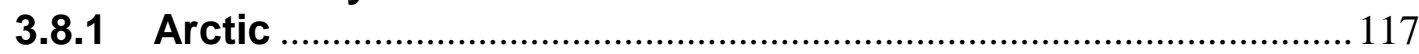

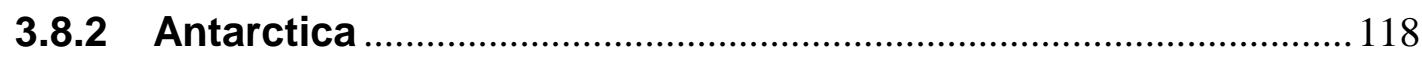

3.9 Brine resistivity calculations

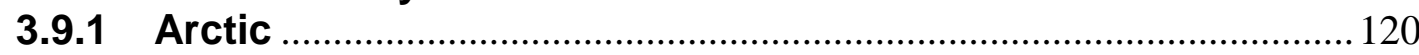

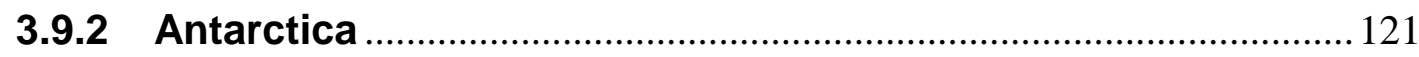

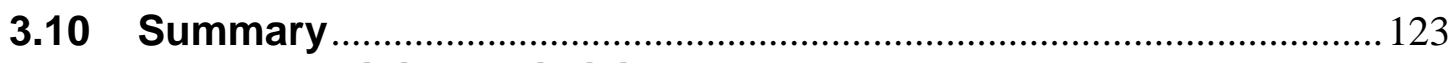

Chapter 4 Obtaining resistivity models ................................................... 127

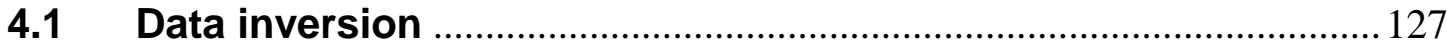

4.1.1 Least-squares optimisation method ............................................. 128

4.1.2 Marquardt-Levenberg modification.............................................. 128

4.1.3 Smoothness-constrained least-squares method .......................... 129

4.1.4 Directly smoothing the model resistivities ..................................130

4.1.5 Robust inversion method ………………................................... 131

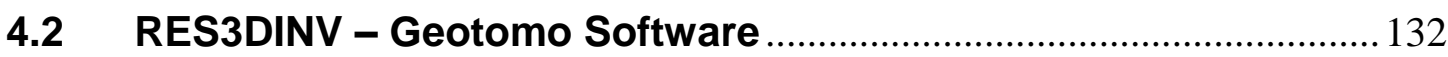

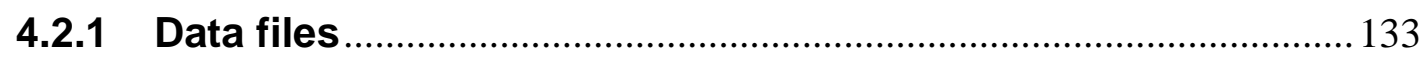

4.2.2 Inversion parameters ...................................................................... 134

4.2.2.1 $\begin{aligned} & \text { Resistance or apparent resistivity as inversion } \\ & \text { variable }\end{aligned}$

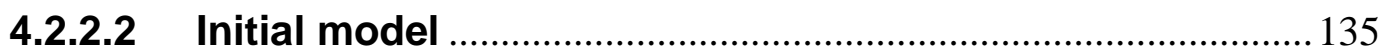

4.2.2.3 Forward modelling method .................................................... 136

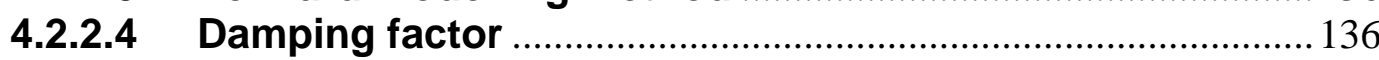

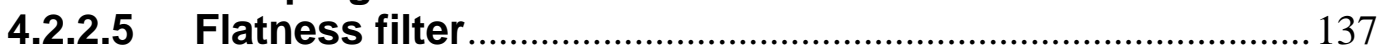

4.2.2.6 Directly smooth model resistivities ……………………….... 137

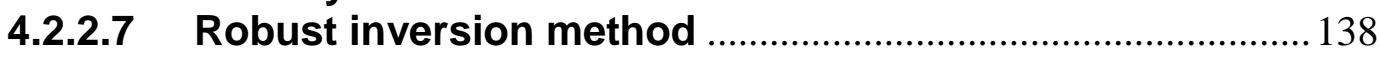

4.2.2.8 Solving the least squares equation......................................... 140

4.2.2.9 Resistivity change: optimum step size ................................ 141

4.2.2.10 Converging the inversion .................................................... 141

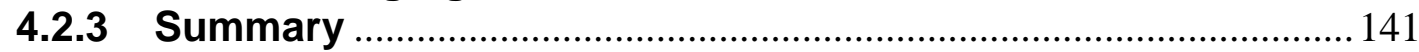

Chapter 5 Analysing resistivity profiles …………................................ 145

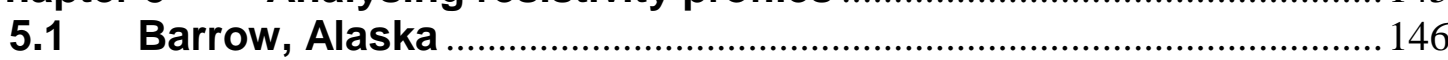

5.1.1 2008 measurements - Jones et al. (2010) .................................... 146

5.1.2 2006 measurements - Ingham et al. (2008) ................................. 158

5.2 McMurdo Sound, Antarctica - 2009................................................ 161

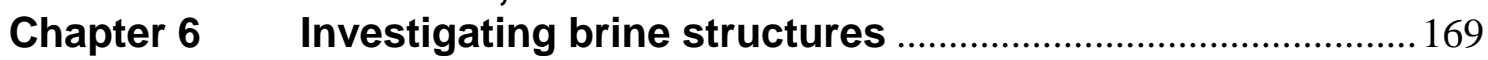

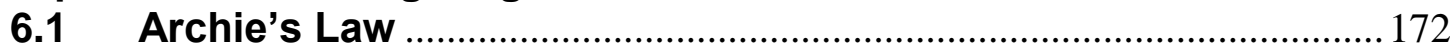

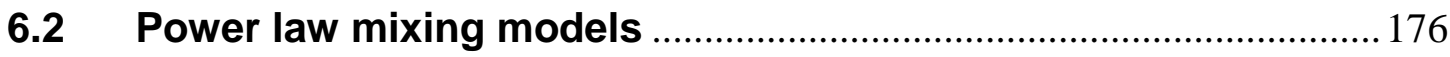

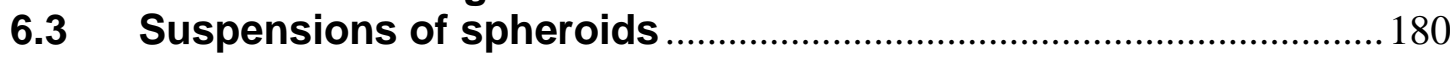

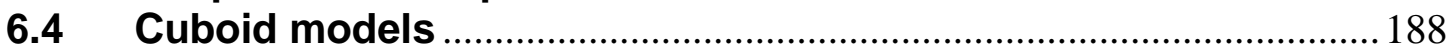




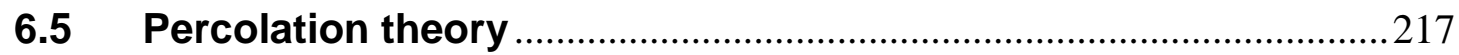

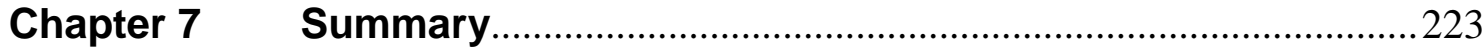

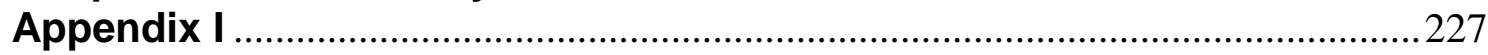

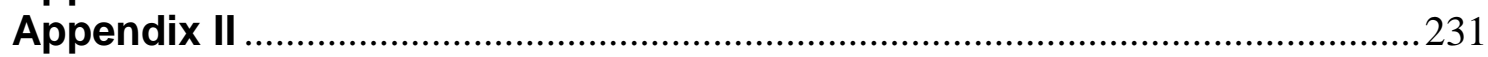

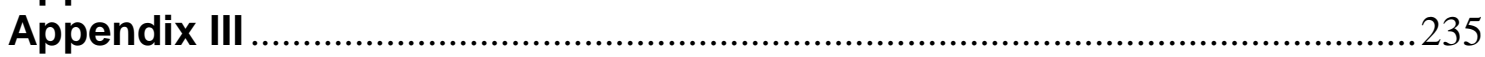

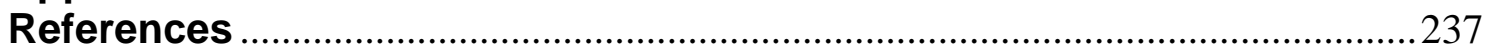





\section{List of figures}

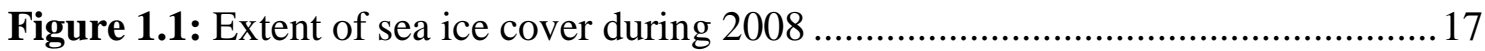

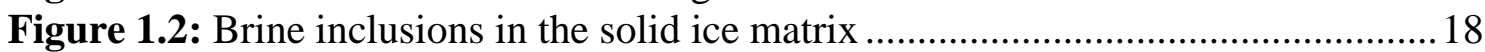

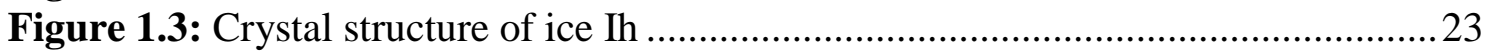

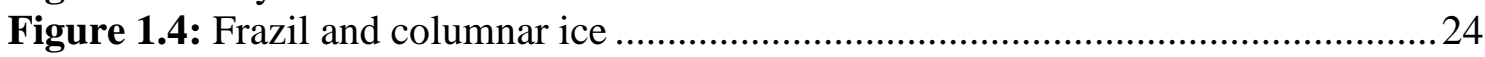

Figure 2.1: Commonly used electrode configurations for surface surveys ................... 46

Figure 2.2: Electrode configuration, with two current and two potential electrodes positioned individually in four boreholes within the ice.........................51

Figure 2.3: Electrode configuration, with two boreholes each containing one current and one potential electrode. .......................................................53

Figure 2.4: Electrode configuration for geometric mean resistivity measurements .......56

Figure 2.5: Point source in one medium separated from another medium by a semi-transparent mirror.

Figure 2.6: Structure consisting of three isotropic resistivity layers, with resistivities $\rho_{0}, \rho_{1}$ and $\rho_{2}$......

Figure 2.7: Current paths between current source $C$ and measurement point $P$, and apparent locations of the image source.

Figure 2.8: Examples of ray paths travelling from $C$ to $P$ via an even number of reflections, with the first reflection being from the bottom boundary ......63

Figure 2.9: Examples of ray paths travelling from $C$ to $P$ via an odd number of reflections, with the first reflection being from the bottom boundary. ..... 65

Figure 2.10: Examples of ray paths travelling from $C$ to $P$, with the first reflection always being from the top boundary

Figure 2.11: Current paths travelling from current electrode $C$, at a depth of $z_{j}$ below the upper boundary to potential electrode $P$, a horizontal distance $a$ away at a depth of $z_{i}$

Figure 2.12: Current paths travelling from current electrode $C$ to potential electrode $P$, both in the seawater.

Figure 2.13: Current paths travelling from current electrode $C$, in the sea ice, to potential electrode $P$, in the seawater.

Figure 2.14: Current paths travelling from current electrode $C$, in the seawater, to potential electrode $P$, in the sea ice.

Figure 2.15: Horizontal resistivity recovered from 3D inversion of synthetic data .......76

Figure 2.16: Geometric mean resistivity recovered from 3D inversion of synthetic data

Figure 2.17: Variation of geometric mean resistivity with depth, recovered from

$1 \mathrm{D}$ inversion of synthetic surface resistivity data.

Figure 3.1: Maps showing the approximate location of the measurement site, in first-year sea ice $\sim 1 \mathrm{~km}$ off the coast of Barrow, Alaska.... 86

Figure 3.2: Maps showing the approximate location of the measurement site, in first-year sea ice $\sim 10 \mathrm{~km}$ off the coast of Ross Island, Antarctica

Figure 3.3: Photos of the electrode strings used to make the cross-borehole resistivity measurements.

Figure 3.4: Field setup of equipment for cross-borehole resistivity measurements in the Arctic.

Figure 3.5: Borehole setup for horizontal resistivity measurements

Figure 3.6: Example of plug and ARES datalogger setup for Arctic field measurements of the horizontal resistivity, labelled rhoh1.

Figure 3.7: Example of plug and ARES datalogger setup for Arctic field measurements of the horizontal resistivity, labelled rhoh2 
Figure 3.8: Borehole setups for geometric mean resistivity measurements. .................95

Figure 3.9: Example of plug and ARES datalogger setup for Arctic field measurements of the geometric mean resistivity, labelled rhomean 1 ......96

Figure 3.10: Example of plug and ARES datalogger setup for Arctic field measurements of the geometric mean resistivity, labelled rhomean2......97

Figure 3.11: Diagram from ARES user manual showing the specification of the

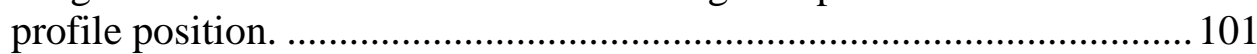

Figure 3.12: Example of the data file produced by the ARES datalogger................... 103

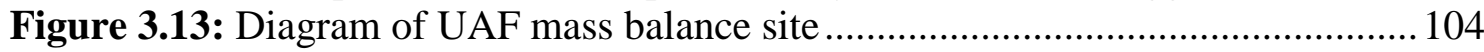

Figure 3.14: Plot of Arctic ice thickness from the 7th Apr to the 17th Jun 2008 ........ 105

Figure 3.15: Plot of snow depth in the Arctic, from the 7th Apr to the 17th Jun 2008

Figure 3.16: Plot of averaged temperature with depth over the period of each measurement set of the 2008 Arctic measurements .

Figure 3.17: Plot of temperatures over the Arctic measurement period 7th Apr to 17 th Jun 2008

Figure 3.18: Plot of temperature with depth over the period of each measurement set of the 2009 Antarctic data.

Figure 3.19: Plot of salinity vs. depth, salinities obtained from ice cores taken by UAF during 2008 Arctic measurements.

Figure 3.20: Evolution of Arctic sea ice salinity profiles

Figure 3.21: Plot of salinity vs. depth, salinities obtained from ice cores taken by K131 members during 2009 Antarctic measurements.

Figure 3.22: Plot of calculated brine volume fraction vs. depth for 2008 Arctic data.

Figure 3.23: Plot of calculated brine volume fraction vs. depth for 2009 Antarctic data.

Figure 3.24: Plot of calculated brine salinity in parts per thousand vs. depth in centimetres for 2008 Arctic data.

Figure 3.25: Plot of calculated brine salinity in parts per thousand vs. depth in centimetres for 2009 Antarctic data.

Figure 3.26: Plot of calculated brine resistivity in ohm meters vs. depth in centimetres for 2008 Arctic data.

Figure 3.27: Plot of calculated brine resistivity in ohm meters vs. depth in centimetres for 2009 Antarctic data.

Figure 4.1: Comparison of models produced using a homogeneous half space as initial model or using the approximate inverse method to obtain the initial model.

Figure 4.2: Effect of directly smoothing the model resistivity on the inversion models.

Figure 4.3: Effect of different model robust constraint cut-off values.

Figure 5.1: Vertical sections through models of the geometric mean resistivity, 2008 Arctic measurements.

Figure 5.2: Vertical sections through models of the horizontal and vertical resistivity, and anisotropy profiles, 2008 Arctic data.

Figure 5.3: Temperature, salinity and brine volume fraction profiles, 2008 Arctic measurements.

Figure 5.4: Line plots of average 2008 data values with depth

Figure 5.5: Plot of resistivity vs. brine volume fraction displaying the behaviour of the Hashin - Shtrikman limits 
Figure 5.6: Plots of the relationships between conductivity and (a) temperature,

(b) brine volume fraction, during the measurement period in the Arctic.

Figure 5.7: $x-y$ and $x-z$ sections of the horizontal resistivity model - displaying the anomalous halos around the electrode strings.

Figure 5.8: Vertical sections through the 3D horizontal resistivity models, as obtained from Fig. 5. in Ingham et al. (2008).

Figure 5.9: Vertical sections through models of the geometric mean resistivity, 2009 Antarctic measurements.

Figure 5.10: Vertical sections through models of the horizontal and vertical resistivity, and anisotropy profiles, 2009 Antarctic data.

Figure 5.11: Temperature, salinity and brine volume fraction profiles, 2009 Antarctic measurements.

Figure 5.12: Line plots of average 2009 data values with depth

Figure 6.1: Plots of formation factor $\left(\rho / \rho_{b}\right)$ vs. brine volume fraction $\left(V_{b} / V\right)$....

Figure 6.2: $\mathrm{Log}-\log$ plots of formation factor $\left(\rho / \rho_{\mathrm{b}}\right)$ vs. brine volume fraction $\left(\mathrm{V}_{\mathrm{b}} / \mathrm{V}\right)$, showing Archie's Law fit to the 2008 Arctic data.

Figure 6.3: $\mathrm{Log}-\log$ plots of formation factor $\left(\rho / \rho_{\mathrm{b}}\right)$ vs. brine volume fraction $\left(\mathrm{V}_{\mathrm{b}} / \mathrm{V}\right)$, showing Archie's Law fit to the 2009 Antarctic data...

Figure 6.4: Plots of formation factor $\left(\rho / \rho_{b}\right)$ vs. brine volume fraction $\left(V_{b} / V\right)$ for Arctic data showing power law trends

Figure 6.5: Plots of formation factor $\left(\rho / \rho_{b}\right) v s$. brine volume fraction $\left(V_{b} / V\right)$ for Antarctic data, showing power law trends

Figure 6.6: Plots of formation factor $\left(\rho / \rho_{b}\right)$ vs. brine volume fraction $\left(V_{b} / V\right)$ for Arctic data, showing Tinga et al. (1973) and Vant et al. (1978) expressions

Figure 6.7: Plots of formation factor $\left(\rho / \rho_{b}\right)$ vs. brine volume fraction $\left(V_{b} / V\right)$ for Antarctic data, showing Tinga et al. (1973) and Vant et al. (1978) expressions

Figure 6.8: Unit cube of a two phase structure, consisting of cubes of a material with conductivity $\sigma_{2}$, within a matrix of material with conductivity $\sigma_{1} \ldots$

Figure 6.9: Plots of formation factor $\left(\rho / \rho_{b}\right)$ vs. brine volume fraction $\left(V_{b} / V\right)$ of both the proposed cubic structures (grey shading) and the measured Arctic data (coloured points)

Figure 6.10: Plots of formation factor $\left(\rho / \rho_{b}\right)$ vs. brine volume fraction $\left(V_{b} / V\right)$ of both the proposed cubic structures (grey shading) and the measured Antarctic data (coloured points)

Figure 6.11: Unit cube of a two phase structure, consisting of vertical cubic columns of a material with a conductivity $\sigma_{2}$, within a matrix of material with conductivity of $\sigma_{1}$.

Figure 6.12: Plots of formation factor $\left(\rho / \rho_{b}\right)$ vs. brine volume fraction $\left(V_{b} / V\right)$ of both the proposed column structure (light grey points) and the measured Arctic data (coloured points)

Figure 6.13: Plots of formation factor $\left(\rho / \rho_{b}\right)$ vs. brine volume fraction $\left(V_{b} / V\right)$ of both the proposed column structure (light grey points) and the measured Antarctic data (coloured points).

Figure 6.14: Unit cube of a two phase structure, consisting of horizontal cubic tubes of a material with conductivity $\sigma_{2}$, within a matrix of material with conductivity of $\sigma_{1}$. 
Figure 6.15: Plots of formation factor $\left(\rho / \rho_{b}\right)$ vs. brine volume fraction $\left(V_{b} / V\right)$ of both the proposed tube structure (dark grey points) and the measured Arctic data (coloured points).

Figure 6.16: Plots of formation factor $\left(\rho / \rho_{b}\right) v s$. brine volume fraction $\left(V_{b} / V\right)$ of both the proposed tube structure (dark grey points) and the measured Antarctic data (coloured points)

Figure 6.17: Unit cube of a two phase structure, consisting of vertical cubic columns and horizontal cubic tubes of a material with conductivity $\sigma_{2}$, within a matrix of material with conductivity of $\sigma_{1}$

Figure 6.18: Plots of formation factor $\left(\rho / \rho_{b}\right)$ vs. brine volume fraction $\left(V_{b} / V\right)$ of both the proposed column and tube structure (black points) and the measured Arctic data (coloured points)

Figure 6.19: Plots of formation factor $\left(\rho / \rho_{b}\right)$ vs. brine volume fraction $\left(V_{b} / V\right)$ of both the proposed column and tube structure (black points) and the measured Antarctic data (coloured points)

Figure 6.20: Unit cube of a two phase structure, consisting of vertical cubic columns, horizontal cubic tubes and cubes of a material with conductivity $\sigma_{2}$, within a matrix of material with conductivity of $\sigma_{1} \ldots . .205$

Figure 6.21: Plots of formation factor $\left(\rho / \rho_{b}\right)$ vs. brine volume fraction $\left(V_{b} / V\right)$ of both the proposed column, tube and cube structure (white points) and the measured Arctic data (coloured points)

Figure 6.22: Plots of formation factor $\left(\rho / \rho_{b}\right) v s$. brine volume fraction $\left(V_{b} / V\right)$ of both the proposed column, tube and cube structure (white points) and the measured Antarctic data (coloured points)

Figure 6.23: Plot of the conductivity of the proposed Arctic column, tube and cube structure for differing conductivities of the ice matrix.

Figure 6.24: Plot of the conductivity of the proposed Antarctic column, tube and cube structure for differing conductivities of the ice matrix.................. 210

Figure 6.25: Plots of the relative sizes, and the trends with time and depth, of the dimensions of the proposed Arctic brine structure.

Figure 6.26: Plots of the relative sizes, and the trends with time and depth, of the dimensions of the proposed Antarctic brine structure.

Figure 6.27: Plot of formation factor $\left(\rho / \rho_{b}\right)$ vs. brine volume fraction $\left(V_{b} / V\right)$ for the vertical resistivity data from the 2008 Arctic measurements.

Figure 6.28: Plot of formation factor $\left(\rho / \rho_{b}\right)$ vs. brine volume fraction $\left(V_{b} / V\right)$ for the horizontal resistivity data from the 2008 Arctic measurements (circles), combined with data from Ingham et al. (2008) (squares).......219

Figure 6.29: Plot of resistance vs. number of resistors changed

Figure 6.30: A $2 \times 2 \times 2$ resistor network where each resistor is itself represented by a $2 \times 2 \times 2$ sub-array of resistors.

Figure 6.31: Plot of formation factor $\left(\rho / \rho_{b}\right)$ vs. brine volume fraction $\left(V_{b} / V\right)$ for resistivity of the $3 \mathrm{D}$ resistor network (50 repeats) and data from measurements on Arctic sea ice. 


\section{List of tables}

Table 1.1: Summary of brine structure statistics obtained from the literature 32

Table 2.1: $T_{i j}$ values for different $z_{i}-z_{j}$ distances

Table 3.1: Summary of the measurements made on first year landfast sea ice in the Chukchi Sea, approximately $1 \mathrm{~km}$ off the coast of Barrow, Alaska over the period 7th Apr to 17th Jun 2008.

Table 3.2: Summary of the measurements made on first year sea ice in McMurdo Sound, approximately $10 \mathrm{~km}$ off the coast of Ross Island, Antarctica over the period 11th Nov to 21st Nov 2009.

Table 3.3: Summary of the measurements made on first year landfast sea ice in the Chukchi Sea, approximately $1 \mathrm{~km}$ off the coast of Barrow, Alaska over the period 7th Apr to 17th Jun 2008.

Table 3.4: Summary of the measurements made on first year sea ice in McMurdo Sound, approximately $10 \mathrm{~km}$ off the coast of Ross Island, Antarctica over the period 11th Nov to 21st Nov 2009.

Table 4.1: Summary of inversion parameters. 



\section{Chapter 1 \\ Introduction}

Sea ice covers a large area of high latitude oceans in the Arctic and Antarctic regions.

This cover begins to form when the temperature of the seawater reaches its freezing point, for seawater with a salinity of 34 practical salinity units (psu) this occurs at approximately $-1.86^{\circ} \mathrm{C}$ (Eicken, 2003). A large fraction of this ice is first-year ice that only survives one season, melting as temperatures warm in spring/summer. However, some of the sea ice survives more than one season, to become multi-year ice. Due to the melting that occurs, the extent of the sea ice varies annually with areas generally ranging from $15.7 \times 10^{6} \mathrm{~km}^{2}$ to $9.3 \times 10^{6} \mathrm{~km}^{2}$ in the Arctic and $18.8 \times 10^{6} \mathrm{~km}^{2}$ to $3.6 \times 10^{6} \mathrm{~km}^{2}$ in Antarctic waters (Dieckmann and Hellmer, 2003), as illustrated by the 2008 sea ice extent in Figure 1.1.

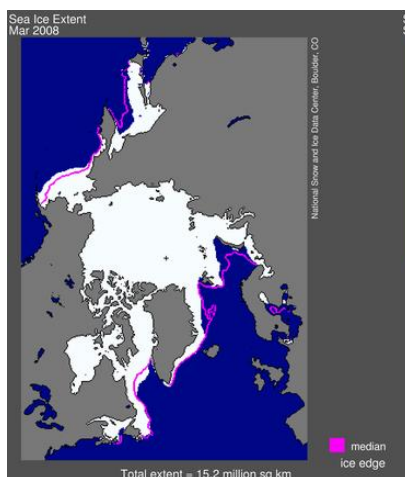

(a)

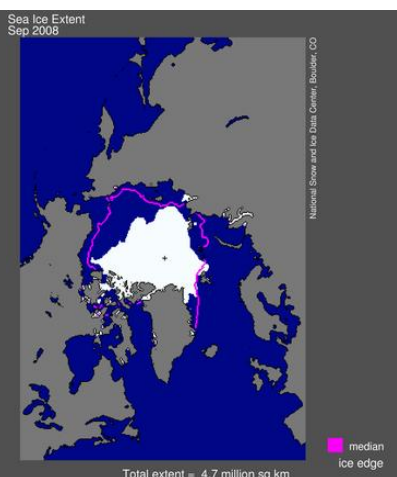

(b)

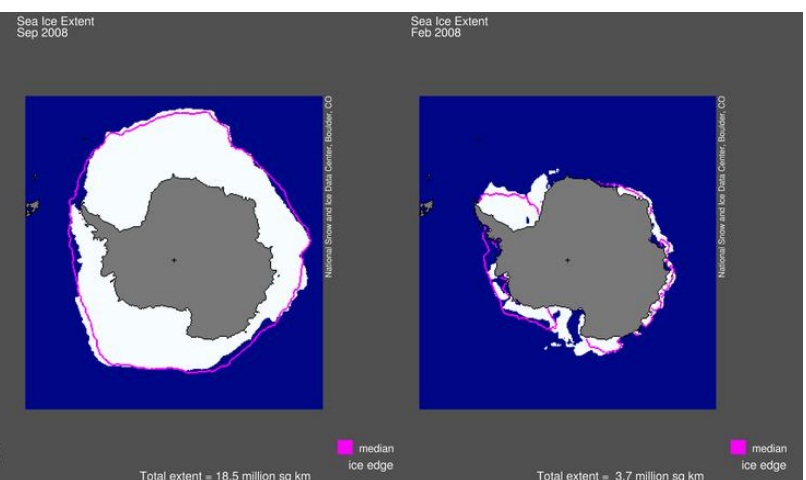

(c) (d)

Figure 1.1: Extent of sea ice cover during 2008; in the Arctic (a) Maximum extent (March) - 15.2 million sq $\mathrm{km}$ (b) Minimum extent (September) - 4.7 million sq km (significantly less than the median extent), and in the Antarctic (c) Maximum extent (September) - 18.5 million sq km (d) Minimum extent (February) - 3.7 million sq $\mathrm{km}$ The pink line indicates the median extent of the ice cover, calculated from 1979-2000 data. Sourced from the National Snow and Ice Data Center (http://nsidc.org/data/seaice_index/)

At full extent approximately 7\% of the Earth's surface is covered by sea ice, as such it is one of the largest biomes on Earth (Dieckmann and Hellmer, 2003). Sitting at the interface between the ocean and the atmosphere, sea ice plays a critical role in Earth's climate (Dieckmann and Hellmer, 2003). For example sea ice regulates the exchange of heat between the ocean and the atmosphere, and its permeability to fluids controls brine exchange with the ocean. Nevertheless, despite the importance of sea ice, a detailed understanding and representation of many of its physical properties is still lacking. In 
this thesis I present a significant advance towards a better understanding of the physical properties of sea ice and their seasonal evolution.

During the formation of sea ice, brine becomes trapped in pockets within the solid ice matrix, as can be seen in Figure 1.2. These range in size from very small sub-millimetre pores to large connected networks of channels several millimetres in diameter and extending over several decimetres. The connectivity of the brine pockets is highly sensitive to temperature and salinity. These brine pockets, in particular their degree of connectivity, are linked to the thermal, optical, mechanical (Eicken, 2003) and biological properties (Krembs et al., 2000) of sea ice. Through these bulk properties sea ice has a determining influence on the global climate (Comiso, 2003) and ecological systems and any changes can impact these systems as well as the use of the ice as a platform for scientific and commercial operations and as a habitat for polar flora and fauna. An understanding of the manner in which the internal microstructure of sea ice changes in response to changes in temperature and salinity is therefore crucial for a fuller understanding of the role that sea ice plays in a range of contexts.

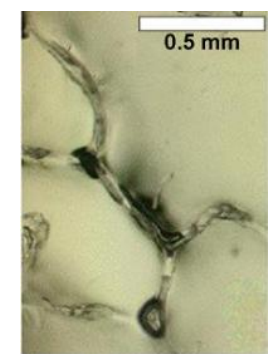

(a)

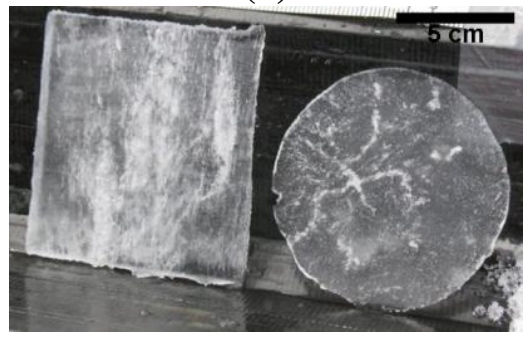

(b)

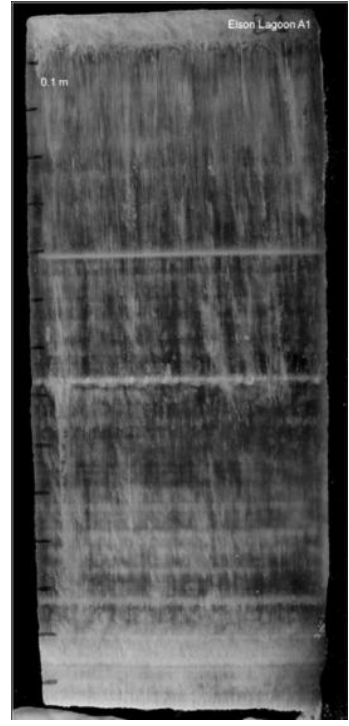

(c)

Figure 1.2: Brine inclusions in the solid ice matrix (a) photomicrograph showing the typical pore structure at $-15^{\circ} \mathrm{C}$ (from Eicken, 2003) (b) photo of thick-sections of an ice core, both vertical (square on left) and horizontal (circle on right), displaying the brine channels (c) photograph of a slab of sea ice obtained from cover of approximately 1.4 m thickness near Barrow, Alaska (from Eicken, 2003). In this slab the vertical brine channels are clearly shown.

For example the existence and connectivity of brine channels in the ice affects the transport of heat (Eicken, 2003). By estimating the latent heat release associated with 
convective overturning and refreezing of brine, Pringle et al. (2007) have estimated that internal brine motion within the ice may somewhat enhance the overall heat flux. Brine inclusions also control the manner in which the ice interacts with electromagnetic radiation (Hallikainen and Winebrenner 1992; Perovich and Gow, 1996; Cherkaeva and Golden, 1998), and thus plays a major role in the interpretation of remote sensing data. Fluid permeability through the ice is important for nutrient transport (Fritsen et al., 1994) which in turn affects the biology of the area. Theoretical calculations of the physical properties of sea ice have generally been based on mixture theories (eg Fricke, 1924; Tinga et al., 1973; Vant et al. 1978; Timco, 1979; Sihvola and Kong, 1988; Chelidze and Gueguen, 1999 and Grimm et al., 2008) and, more recently, on percolation theory (eg. Golden et al., 1998, 2007 and Golden, 2003). Nevertheless, despite the relevance of the microstructure of sea ice to determining its role in the global climate system, there are few field measurements of the temporal variation in the physical properties of sea ice with which such theoretical calculations can be compared.

In theory the internal structure of sea ice can be studied using any transport property to which the brine and ice components contribute differently (Ingham et al., 2008). In fact the microstructure, thermal evolution, fluid transport and permeability of sea ice brine inclusions have been studied by several authors using, for example, magnetic resonance techniques (eg. Callaghan et al., 1999; Eicken et al., 2000; Mercier et al., 2005), bailtest and fluorescent tracer methods (eg. Freitag and Eicken, 2003), x-ray computed tomography (eg. Kawamura, 1988, Pringle et al., 2009b), and impedance measurements (eg. Notz and Worster, 2008; Pringle et al., 2009a).

However, sea ice is a complex anisotropic medium composed of a solid ice matrix, trapped air, brine, and, at low temperatures, precipitated salts. The proportions and arrangement of these components vary with depth, temperature and with the history of the ice. Thus, in practice there are severe difficulties in making accurate measurements as most methods inevitably disturb the ice from its natural state. For example, investigation of the internal structure of sea ice by removing a sample from the ice cover can alter the properties under study. This change in properties can be caused by the interconnections between the brine pockets, which during the removal process can allow brine drainage (especially in the high porosity ice near the bottom of the cover). This drainage can be quite marked at warmer temperatures and may still be significant below $-23^{\circ} \mathrm{C}$ (Addison, 1969). Likewise, growing sea ice in the laboratory will not 
necessarily create ice with the same properties as natural sea ice, especially in relation to crystal structure and brine inclusion shape and distribution. Furthermore, insertion of large probes into the ice cover may induce artificial brine motion or affect the growth of the sea ice.

Since there is a large contrast between the resistivity of the brine inclusions and solid ice matrix, measurements of electrical resistivity should yield information on sea ice microstructure, and through this, indirect probing of other key ice properties. Direct current (DC) geoelectric soundings, widely used in shallow geophysical studies, are generally made using surface electrodes and therefore do not disturb the natural structure of the sub-surface. Simple surface resistivity measurements use two pairs of electrodes; one pair of electrodes introduces an electric current into the medium, the resulting potential difference is then measured between the other pair. However, due to the preferential vertical alignment of brine inclusions, the bulk resistivity of sea ice is anisotropic with the vertical resistivity $\left(\rho_{v}\right)$ being lower than horizontal resistivity $\left(\rho_{H}\right)$. The degree of anisotropy is defined by $\lambda=\sqrt{\rho_{v} / \rho_{H}}$ the coefficient of anisotropy. Surface resistivity soundings, on anisotropic media, can be shown (e.g. Bhattacharya and Patra, 1968) to only be sensitive to the geometric mean resistivity $\left(\rho_{m}=\sqrt{\rho_{v} \rho_{H}}\right)$ of the medium. This has been clearly noted in previous resistivity measurements made on sea ice (e.g. Thyssen et al., 1974; Timco, 1979; Buckley et al., 1986; Ingham et al., 2008) which have also shown that surface soundings underestimate the true thickness of the anisotropic sea ice cover. This makes the interpretation of soundings in terms of variation of geometric mean resistivity with depth problematic.

One technique that overcomes the issues of surface measurements while still retaining the in situ constraint is that of cross-borehole electrical resistivity tomography. Ingham et al. (2008) have demonstrated that this technique can be used to measure the horizontal component of the anisotropic resistivity structure of sea ice. The technique involves making resistivity measurements using vertical strings of evenly spaced electrodes inserted into boreholes, drilled in the ice while it is relatively thin. These boreholes subsequently re-freeze around the electrode strings, which are further embedded into the growing ice sheet. Although insertion of electrode strings, and their subsequent freezing in, disturbs the ice structure immediately adjacent to the strings, the 
bulk of ice between the boreholes is undisturbed and it is through this that current passes. Such measurements therefore largely sample ice in its natural state.

The aim of this PhD study was to build on and extend the Ingham et al. (2008) study of first-year sea ice near Barrow, Alaska. The present project looks at further developing the use of the non-destructive in situ technique of cross-borehole resistivity tomography to determine the three dimensional anisotropic resistivity structure of first year sea ice in both the Arctic and the Antarctic. The horizontal, geometric mean and vertical resistivity structures of sea ice are obtained from measurement, numerical modelling and calculation. These resistivity structures provide an insight into the internal brine structure as it changes with depth and with changes in temperature and salinity. Simple models of this brine microstructure are constructed that match the resistivity data. Furthermore, it was possible to compare the structure of ice formed in the differing environments of the Arctic and Antarctic, using the resistivity data gathered in the two separate regions. The measurement based picture obtained from this study, can be used to enhance the present theoretical models of sea ice microstructure. This research also shows the effectiveness of the measurement technique as a non-destructive method of observing the brine structure of sea ice, and hence provides a way of investigating other various properties of the sea ice.

This thesis is structured as follows. Chapter one contains the introduction and provides a brief overview of sea ice, as well as more detailed discussion of the brine component and electrical structure of sea ice. Chapter two focuses on electrical resistivity measurements and theoretical considerations associated with developing techniques suitable for obtaining values for the horizontal, geometric mean and vertical resistivities of first year sea ice. An overview of the field procedure at the two measurement sites is given in chapter three. Additionally this chapter provides plots of temperature, salinity and brine volume fraction with depth, important properties of the sea ice which aide in interpretation of the resistivity measurements. Chapter four briefly looks at the 3D inversion process, the software used and the programme parameters altered during inversion of the data. The horizontal, geometric mean and vertical resistivity structures for all measurement sets in both the Arctic and Antarctica are displayed in chapter five. Here the results are analysed and interpreted in terms of salinity and temperature measurements and calculated brine volume fractions. In chapter six attempts are made at defining a brine structure that matches the resistivity structures obtained from 
inversion of the data. Initially mixture theory models suggested by other authors (eg Fricke, 1924; Tinga et al., 1973; Vant et al. 1978; Timco, 1979; and Grimm et al., 2008) are considered followed by the creation of our own cuboid structured model. Final discussion and summary are found in chapter seven. 


\subsection{Sea ice overview*}

\subsubsection{Formation}

Natural seawater usually has a salinity of about $35 \%$, with the most abundant salt ions being $\mathrm{Cl}(19 \%), \mathrm{Na}(11 \%), \mathrm{SO}_{4}(2.6 \%)$ and $\mathrm{Mg}(1.3 \%)$ though many others are present in smaller quantities (Addison, 1969). The freezing point of seawater is salinity dependent but typically around $-1.86^{\circ} \mathrm{C}$ (Eicken, 2003). This is lower than the freezing point of pure water because of the presence of dissolved salts.

As the seawater cools to the freezing temperature ice crystals form throughout the upper layers and are kept in suspension until a surface layer of ice slush (eg. Figure 1.4a) builds up, reducing mixing. The structure of these crystals has a hexagonal symmetry, with the principal crystallographic axis (c-axis) corresponding to the axis of maximum rotational symmetry (as shown in Figure 1.3 below). The c-axis is normal to the basal plane (defined by the crystal a-axes), which coincides with the plane of fastest ice growth.

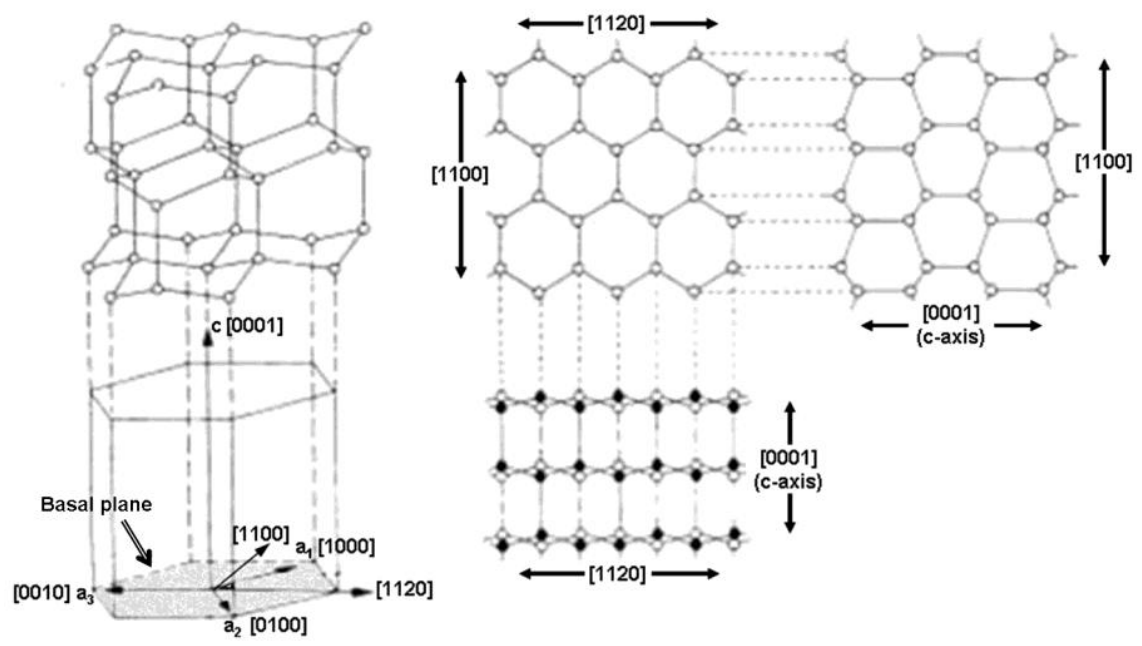

Figure 1.3: Crystal structure of ice Ih (altered from Weeks and Ackley, 1986), showing; hexagonal symmetry, the principal crystallographic axis (c-axis) and the basal plane along which the greatest rate of growth occurs. Coordinate system is a tetrahedral geometry.

\footnotetext{
* The text, Sea Ice: An introduction to its Physics, Chemistry, Biology and Geology by Blackwell Science $L t d$ provides a nice introduction to sea ice; its microstructure, properties and growth (especially the chapter by Eicken) and a lot of the material in this section has been gained through the reading of this book.

$\S$ Salinity measurements in parts per thousand (ppt or \%o) can be assumed, for standard seawater composition, to coincide with practical salinity units (psu) between 1\%o and 42\%o (Eicken, 2003).
} 
The small crystals forming in the surface slush layer have more or less random c-axis orientations. These crystals merge and build up and the slush layer becomes more solid. Once a continual ice sheet has formed at the sea surface, congelation growth occurs with seawater beginning to freeze to the underside of the ice in somewhat calmer conditions. The uppermost region of the growing ice cover, grown in more turbulent conditions than the ice below, is granular in structure with random crystal orientation, and is often termed frazil ice. Since growth of the ice crystal is favoured along the basal plane (i.e. perpendicular to the c-axis), crystals having this plane parallel to the temperature gradient grow at the expense of those less favourably orientated. This produces a 'transition layer' below which the crystals tend to show a horizontal c-axis orientation and to grow in the form of vertical columns (Figure 1.4b). The ice formed from these vertically elongated crystals has a columnar rather than granular texture and is referred to as columnar ice.

Generally in the study of sea ice an 'ice crystal' refers to a region of ice made up of individual ice 'grains' all with the same c-axis orientation. Thus a single crystal of sea ice is made up of a substructure of evenly spaced parallel grains. When thin sections of sea ice are viewed through cross polarisers, grains with the same c-axis orientation will show the same brightness/colour. Grains with different c-axis orientation will have different colours depending on the intensity of transmitted light and the variation of the velocity of light with wavelength (Pounder, 1965). Thus using cross polarisers provides a good way of observing the shape of the ice crystals, as can be seen in Figure $1.4 \mathrm{~b}$ below.

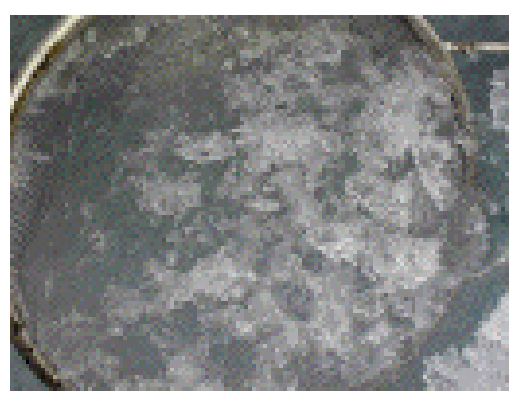

(a)

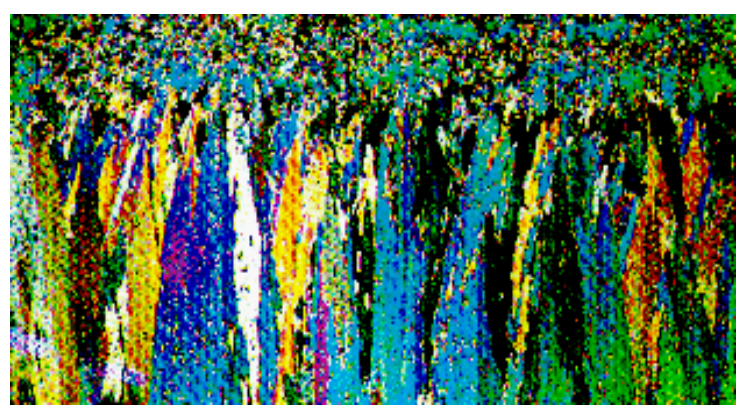

(b)

Figure 1.4: Frazil and columnar ice (a) photo of frazil ice slush forming on the surface of seawater (from University of Alaska Fairbanks (UAF) floating ice group's ice glossary http://www.gi.alaska.edu/ eicken/he_teach/GEOS 615icenom /iceglossary/iceglossary.htm) (b) Vertical section of sea ice (from http://www.hokudai.ac. jp/lowtemp/ sirl/sirl-e.html), a surface layer of frazil ice can be seen over the longer, vertically aligned crystals of columnar ice 
As seawater freezes the dissolved salts are rejected from the newly forming ice, this process enhances the salinity in the surrounding seawater. Some of this higher salinity brine is trapped within pockets in the solid ice as it grows downwards. However a sizeable fraction of the salt is rejected into the water underneath. Thus natural sea ice shows salinities lower than the typical 35\% of seawater, with a range from less than $4 \%$ for older ice to as high as 20\%o for rapidly frozen new ice (Addison, 1969).

As the ice grows downwards salt builds up ahead of the advancing seawater/ice interface, increasing the salinity of a thin layer (a few millimetres to a few centimetres in thickness) of seawater. At the same time as the concentration of the salt in the brine increases, the brine's freezing point decreases (Addison, 1969; Eicken, 2003). As the ice/water interface must be at the respective melting/freezing point, the increase in salt along the interface is matched by a decrease in temperature, relative to the underlying seawater. The transport of heat, from the warmer seawater to the ice, is faster than the transport of the salt, away from the ice. Therefore a region ahead of the seawater/ice interface, which is cooled due to upward heat transport, but has not received a corresponding influx of salt from above, is said to be supercooled. That is, the temperature in this region is lower than the freezing point associated with its salinity. Any small piece of ice that projects into the supercooled zone is at a growth advantage. Consequently, the seawater/ice interface can grow into ordered patterns of lamellar bulges. When fully developed, as in the case of ordinary columnar sea ice, the lamellar interface consists of sub millimetre thick blades of ice, separated by narrow channels of brine.

As more ice is added at the water interface, the temperature of this region drops, consequently the ice lamellae thicken and the fraction of liquid decreases. Eventually, this region is incorporated into the interior. Ice bridges form between the lamellae joining them up and consolidating the region into lower porosity sea ice. This traps the remaining brine in pockets between the ice grains that make up ice crystals forming intracrystalline layers. 


\subsubsection{Properties}

\subsubsection{Thermal}

The growth rate of ice is determined by the energy or heat balance at the seawater/ice interface. The thermal properties of the ice control the amount of heat transferred through the ice and thus the growth rate and equilibrium thickness of the ice. As the thermal conductivity of brine is approximately four times smaller than that of pure ice, the volume fraction of brine and its microstructural arrangement exert a significant influence on the bulk sea ice thermal conductivity (Pringle et al., 2007; Eicken, 2003). Convective motion within the brine inclusions has been observed (Trodahl et al., 2000) and can contribute several $\mathrm{Wm}^{-2}$ to the total heat transfer through the ice column (Eicken, 2003).

\subsubsection{Mechanical}

The strength of the sea ice is to a large extent controlled by the volume fraction of gas and brine, as these components do not contribute to the overall mechanical strength of the ice. Once the air temperature rises to above approximately $-10^{\circ} \mathrm{C}$, the brine pockets rapidly begin to increase in size, causing a decrease in ice strength (Timco and Johnston, 2002). The ratio of the basic strength parameters, compressive and tensile, determines the behaviour of the ice under pressure. A high compressive to tensile strength ratio and low fracture toughness, leads to brittle ice, as in the case with cold, freshwater ice. On the other hand, with a lower ratio the ice may be quite ductile, as in the case of flooded sea ice. Knowledge of the mechanical properties is important for determining the use of the sea ice as a platform and for understanding its interaction with other structures.

\subsubsection{Optical}

The optical properties of sea ice, such as the albedo (the fraction of incident irradiance that is reflected from the surface of the medium (Perovich, 1996)), strongly influence the spring warming and summer melt rates. Indeed, the ice-albedo feedback mechanism plays a key role in the heat and mass balance of the sea ice cover (Grenfell and Perovich, 2004). The amount of irradiance reflected from the medium is determined by the scattering and absorption of the light. Therefore the internal structure, especially the brine and air inclusions, play an important role in determining the optical properties of sea ice. 


\subsubsection{Electrical}

The electrical properties of sea ice are best understood by considering first the pure ice matrix and then the influence of impurities. Due to the permanent dipole moment of the water molecules, chemically pure ice is a lossy dielectric (Mathews and Clarke, 1963) or a partially conducting medium. The dielectric relaxation behaviour of pure ice can be described by a Debye dispersion with a single relaxation time which varies with temperature (Addison, 1969). Conduction is facilitated in pure ice through ionic processes with hydrogen ions or protons acting as the charge carriers. Two kinds of protonic point defects can occur in the ice lattice to allow charge movement. Rotation of a water molecule around one of its four bonds produces a site with no protons (Bjerrum L-defect) and another with two (Bjerrum D-defect). Movement of a proton along hydrogen bonds creates $\mathrm{H}_{3} \mathrm{O}^{+}$(charge surplus) and $\mathrm{OH}^{-}$(charge deficit) ionic defects (Gränicher et al., 1957; Hobbs, 1974; Grimm et al., 2008). DC conduction requires the motion of both Bjerrum and ionic defects, as the polarisation induced by passage of one kind of defect must be reversed by the other (Hobbs, 1974; Grimm et al., 2008).

There are three main classifications of impurities in ice (Mathews and Clarke, 1963):

(i) Those existing integrally in the crystal lattice. In this situation the impurity molecule replaces a water molecule but uses the same bonds. The presence of ions in the crystal is somewhat analogous to that of doping agents in semi-conductors (Addison, 1969). With impurities that are able to substitute into the lattice structure introducing protonic point defects (Grimm et al., 2008). Wolff et al. (1997) suggest that only $\mathrm{H}^{+}, \mathrm{NH}_{4}{ }^{+}$(or $\mathrm{NH}_{3}$ ) and $\mathrm{Cl}^{-}$(or $\mathrm{HCl}$ ) are able to enter the ice lattice. Due to the covalent radius for $\mathrm{N}$ and $\mathrm{Cl}$ being similar to that of $\mathrm{O}$ atoms, the mentioned ions are able to replace existing atoms in the ice. It is expected that large anions such as $\mathrm{NO}_{3}{ }^{-}$or $\mathrm{SO}_{4}{ }^{2-}$ don't play any role nor would cations such as $\mathrm{Na}$ or Ca have an effect (Wolff et al., 1997). The DC conductivity of a salt-doped ice will always remain very small, because the $\mathrm{H}_{3} \mathrm{O}^{+}$are hardly augmented. This is confirmed by the DC conductivity analysis of Grimm et al. (2008), which derived an average value of $\sim 5 \times 10^{-12} \mathrm{Sm}^{-1}$ for ice at $-65^{\circ} \mathrm{C}$.

(ii) Those existing in crystal voids or along grain boundaries as crystallised salts.

(iii) Those existing in the same voids but as ions in solution. In this situation charges are free to migrate.

In Jaccard theory (Jaccard, 1964 and Petrenko, 1993), it is assumed that intra-granular conductivity dominates, with $\mathrm{DC}$ conductivity controlled by the $\mathrm{H}_{3} \mathrm{O}^{+}$ionic defects. 
However, Wolff et al. (1997), in their study of polar ice, suggest there is evidence that DC conductivity of impure ice may be controlled by grain boundary processes especially in the case of sea ice, where brine is present at these boundaries. Toyama et al. (2001) studied the DC electrical conductivity behaviour of sea ice, noting that down to a temperature of about $-54^{\circ} \mathrm{C}$ the $\mathrm{DC}$ conductivity of sea ice is controlled by the brine distribution existing at the grain boundaries. Morey et al. 1984 have shown the complex dielectric constant of sea ice to be a function of the brine volume and the orientations of the brine inclusions.

The dielectric properties of sea ice determine the propagation and attenuation of electromagnetic (EM) waves through the ice. Thus an understanding of these properties is important in interpreting the return from remote sensing techniques.

\subsubsection{Arctic vs. Antarctic}

The main differences observed between Arctic and Antarctic sea ice arise from the differing environments. The Arctic is mostly ocean surrounded by land masses, while Antarctica is a land mass surrounded by open oceans.

Snow cover acts as an insulator on the surface of the ice, slowing growth. In the Antarctic where the surrounding seas provide a permanent moisture source the snow can be thicker than $0.5 \mathrm{~m}$ (Haas, 2003), while in the Arctic the snow cover is much thinner. In the Southern ocean the mean oceanic heat flux is about $40 \mathrm{Wm}^{-2}$ while in Arctic waters the heat flux is around 10 times less at $4 \mathrm{Wm}^{-2}$ (Haas, 2003). As a consequence of the thick snow cover and high ocean heat fluxes, Antarctic ice may only grow to a thickness of $0.5 \mathrm{~m}$ to $0.7 \mathrm{~m}$ (Eicken, 2003) in a single year and melt at its underside even during winter (Haas, 2003). On the other hand, in a single year sea ice in the Arctic can grow to a thickness of $1.8 \mathrm{~m}$ (Eicken, 2003). Thicker snow cover can cause greater ice growth if the snow cover is sufficient to depress the ice surface below sea level leading to the formation of snow-ice (Eicken, 2003).

A significant amount of the ice in the Arctic Ocean drifts for three to six years (Haas, 2003) growing thicker from thermodynamic growth over several winters. This ice motion is confined by coasts where the ice can converge and thicken further by deformation. In contrast most Antarctic ice melts during the summer and seldom 
becomes older than a year. Most of the ice drift around Antarctica is divergent and towards the open seas. This causes the opening of leads and the addition of thin new ice to the thickness distribution.

In the Arctic with its surrounding land masses, frazil ice only forms in marginal seas, leads and other openings in the ice pack. Thus most, between $60 \%$ and $80 \%$ (Eicken, 2003), of the cover is composed of columnar ice. In Antarctica, on the other hand, the effects of open ocean; higher wind speeds, swell penetrating from lower latitudes and the larger number of openings in the pack ice, means that in some regions the majority, as much as $60 \%$ to $80 \%$ of the total thickness, of the ice is made up of frazil ice (Eicken, 2003). Furthermore in parts of the Antarctic larger ice platelets are found to grow at depths, in water parcels supercooled through interactions with the melting Antarctic ice shelves (Eicken, 2003). This process is capable of generating large volumes of crystals that can grow as large as $10 \mathrm{~cm}$ across and a few mm's thick and float towards the surface accumulating in layers several meters thick (Eicken, 2003). These layers can be incorporated into the ice cover as it grows downwards (McGuiness et al., 2009; Dempsey et al., 2010), providing a mechanism for increased thickness of sea ice in regions where platelet ice occurs. As the southern end of McMurdo Sound is bounded by McMurdo Ice Shelf (a region of the Ross Ice Shelf), it is one area where platelet ice is commonly observed and studied (Leonard et al., 2006; McGuiness et al., 2009; Dempsey et al., 2010).

Most of the ice in the Arctic is located at latitudes north of $70^{\circ} \mathrm{N}$ (Haas, 2003), with extent in lower latitudes limited by land masses. However, in Antarctica a significant mass of ice extends north into much lower latitudes, as far as $60^{\circ} \mathrm{S}$ (Haas, 2003). Thus air temperatures, total incoming solar radiation and the length of summer are generally higher in Antarctica. However the Antarctic ice sheet is a giant cold reservoir, and the sea ice region is well isolated from lower latitudes by the Antarctic Circumpolar Current so that warm and moist air advection are not as important as they are for the Arctic (Haas, 2003). There, despite its high latitude, strong surface melting occurs in summer even at the North Pole (Haas, 2003). 


\subsection{The brine component of sea ice}

The previous section discussed how brine inclusions become incorporated into the solid ice matrix as sea ice grows and the influence of this brine component on many properties of the sea ice cover. Thus an understanding of the density, dimension, orientation, connectivity and evolution of the brine phase is important in the study of the sea ice system.

\subsubsection{Structure}

As described earlier typical columnar ice crystals are composed of vertically elongated grains of pure ice separated by parallel layers of brine inclusions. Due to gravity and vertical thermal gradients the brine inclusions in sea ice are normally orientated vertically. Many authors have studied the brine microstructure of sea ice, using a range of techniques from analysing optical images (eg. Perovich and Gow, 1991, 1996; Shokr and Sinha, 1994; Light et al., 2003) to magnetic resonance techniques (eg Eicken et al., 2000) to x-ray computed tomography (eg Pringle et al., 2009b).

The substructure of the ice crystals appears to depend inversely on the growth rate of the ice (Lofgren and Weeks, 1969; Nakawo and Sinha, 1984). Thus as the growth rate decreases as the ice thickens, the grain width should increase with depth within the ice cover. Pounder (1965) gives values of $0.5 \mathrm{~mm}$ to $0.6 \mathrm{~mm}$ for the average thickness of the ice grains. A larger range of values is given by Nakawo and Sinha (1984), who measured brine layer spacings of $0.4 \mathrm{~mm}$ to $0.9 \mathrm{~mm}$ on an ice core sample taken from Eclipse Sound, Baffin Island, Canada. Using X-ray computed tomography Pringle et al. (2009b) obtains slightly lower values of $0.2 \mathrm{~mm}$ to $0.5 \mathrm{~mm}$ for the typical thickness of the ice lamellae between brine layers. The dimensions of the ice crystals themselves are of the order of a few to tens of millimetres across (Shokr and Sinha, 1994; Eicken, 2003).

On a large scale, visible to the naked eye, brine channels are easily defined within ice sections (eg. Figure 1.2 b and c). Morey et al. (1984) describe ribbon like drainage routes, called brine feeder channels, sloping upward at angles of $30^{\circ}$ to $60^{\circ}$, radiating out from larger vertical drainage tubes. These drainage tubes extend to the bottom of the 
ice and have been found, by Morey et al. (1984), to be most often spaced about $10 \mathrm{~cm}$ apart. The channels are thinnest higher up in the ice cover and become thicker as one moves to the bottom of the ice, providing a route for brine drainage to the sea. In their study of first year sea ice cores, obtained in Resolute Passage off Barrow Strait, Northwest Territories, Canada, Shokr and Sinha (1994) observed drainage channels of approximately $4 \mathrm{~mm}$ width in columnar ice and spherical brine inclusions in frazil ice with dimensions varying from fractions of a millimetre to $2 \mathrm{~mm}$.

Returning to the small scale of brine inclusions located between ice grains early investigations indicate a temperature dependent average diameter of $0.05 \mathrm{~mm}$ (Pounder, 1965, Addison, 1969). Addison (1969) also notes that liquid brine is found along grain boundaries.

Perovich and Gow (1991), (1996) analyse photomicrographs of horizontal thin sections of ice using a personal computer-based image-processing system to determine the number, size and statistics of brine inclusions in both saline ice grown in ponds and sea ice in the Beaufort sea at Barrow, Alaska. From a sample of young saline ice, with a predominant columnar structure, the brine inclusions had a mean cross-sectional area of $0.028 \mathrm{~mm}^{2}$ and mean major and minor axes of $0.1 \mathrm{~mm}$ and $0.08 \mathrm{~mm}$ respectively. In firstyear sea ice cover the frazil ice within the upper most regions of the cover contained larger brine inclusions with mean major axes lengths between $0.30 \mathrm{~mm}$ and $0.37 \mathrm{~mm}$ and mean areas of $0.031 \mathrm{~mm}^{2}$ to $0.056 \mathrm{~mm}^{2}$. The brine inclusions in the columnar ice beneath ranged from mean major axes lengths of $0.26 \mathrm{~mm}$ to $0.24 \mathrm{~mm}$, with mean areas of between $0.019 \mathrm{~mm}^{2}$ and $0.023 \mathrm{~mm}^{2}$. The top of the first year sea ice also had the lowest number of brine inclusions $\left(\sim 1\right.$ per $\left.\mathrm{mm}^{3}\right)$, but the number density increased at the base of the ice $\left(\sim 4.5\right.$ per $\left.\mathrm{mm}^{3}\right)$. In the middle of the ice the number density of brine inclusions varied between 1.6 and 2.2 per $\mathrm{mm}^{3}$.

Light et al. (2003) also analysed photomicrographs of samples from ice cores extracted from shorefast ice near point Barrow, Alaska. In this study they were able to resolve smaller inclusions than Perovich and Gow (1991, 1996). Brine inclusion lengths were found to range from $0.01 \mathrm{~mm}$ to $8 \mathrm{~mm}$, diameters from $0.01 \mathrm{~mm}$ to $0.230 \mathrm{~mm}$, and number densities averaged about 24 inclusions per $\mathrm{mm}^{3}$. This number density is significantly larger than other quoted values, probably due to the ability to detect small brine inclusions. Many of the inclusions did not have a circular cross-section, however, the 
authors note no clear indication that the horizontal aspect ratios were elongated in a particular direction.

From studies of ice and salt hydrate systems Grimm et al. (2008) represent the brine channel width as $d=0.34 D \phi_{W}^{3 / 4}$ (where $D$ is the diameter of the ice grains, which are modelled as truncated semi-regular octahedra; and $\phi_{W}$ is the volume fraction of unfrozen water). For ice grains of diameter $D=1 \mathrm{~mm}$, the representative brine channel width is in the micron range just above the eutectic temperature of $\mathrm{CaCl}_{2}, \mathrm{NaCl}$ and $\mathrm{HCl}$. To avoid confusion with terminology, it should be noted that the use of the term brine 'channel' by the authors (Grimm et al., 2008) describes a structure with a width significantly smaller than the dimensions normally associated with brine channels, including those cited by other authors mentioned in this section.

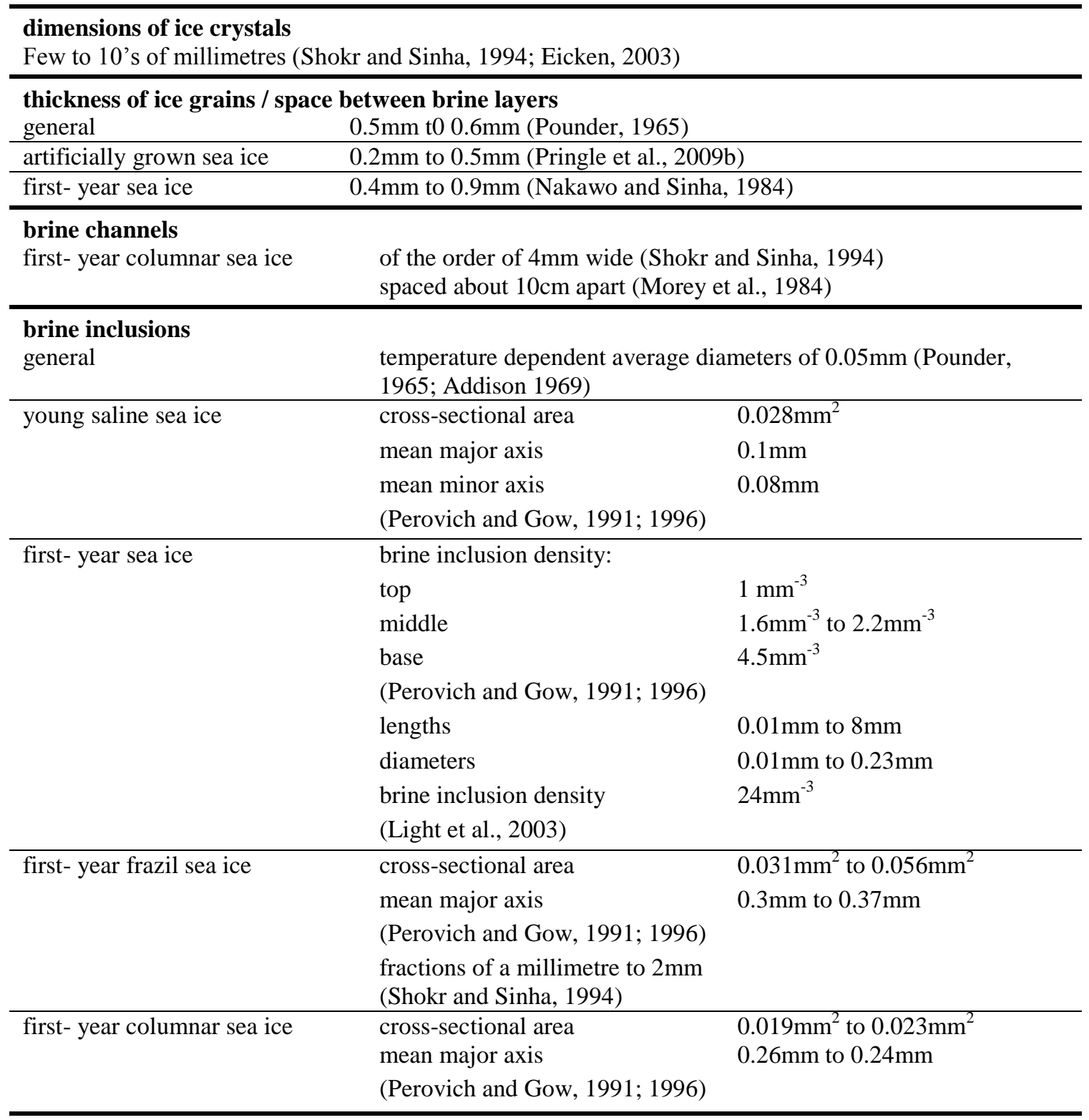

Table 1.1: Summary of brine structure statistics obtained from the literature 


\subsubsection{Evolution}

Generally as temperature increases brine salinity decreases (Haas et al., 1997). However, the amount of brine increases with increasing temperature (Addison, 1969). These observations can be explained, as when the ice melts from the brine inclusion walls, increasing their size and connectivity and thus the amount of brine, it dilutes the brine solution in the inclusion, thus decreasing its salinity. Conversely, as the ice cools the concentration of the brine must increase if it is not to freeze. This increase in concentration occurs when pure water from the brine inclusion freezes to the surrounding ice lattice, reducing the size of the inclusion.

Many of the authors mentioned above have investigated these temperature effects on the brine microstructure. Perovich and Gow $(1991,1996)$ warmed the ice samples from $-20^{\circ} \mathrm{C}$ to $-1^{\circ} \mathrm{C}$, during this warming the brine volume of young saline ice increased from $1.9 \%$ to $37.4 \%$. In first year ice samples they observed a significant increase in the mean area of the brine inclusions when brine volumes increased from $20 \%$ to $40 \%$. At the same time there was a decrease in the number density of the brine inclusions. This indicated that as the ice warmed individual brine inclusions were coalescing to form fewer, larger inclusions. It was also noticed that the brine inclusions elongated in the horizontal direction as temperatures increased.

Eicken et al. (2000) used magnetic resonance techniques to image the evolution of the pore microstructure of sea ice samples from the Chukchi Sea near Barrow, Alaska. They observed changes in the brine structure of the uppermost layers of columnar ice, composed of small crystals with no preferred horizontal alignment of c-axes, as the ice was warmed from $-21^{\circ} \mathrm{C}$ to $-10^{\circ} \mathrm{C}$ to $-6^{\circ} \mathrm{C}$. The mean size of major/minor axes of the brine inclusions in horizontal sections were observed to increase with increasing temperature from $0.34 \mathrm{~mm} / 0.22 \mathrm{~mm}$ to $0.34 \mathrm{~mm} / 0.23 \mathrm{~mm}$ to $0.38 \mathrm{~mm} / 0.25 \mathrm{~mm}$. In the vertical sections the mean major/minor axis increased from $0.42 \mathrm{~mm} / 0.21 \mathrm{~mm}$ to $0.43 \mathrm{~mm} / 0.22 \mathrm{~mm}$ to $0.70 \mathrm{~mm} / 0.26 \mathrm{~mm}$. From a comparison of these dimensions it was noted that the increase in size of the inclusions occurred mostly in the vertical direction. The number density of inclusions in the vertical sections was observed to decrease, from 0.75 to 0.58 per $\mathrm{mm}^{3}$ with increasing temperature, as individual pores joined together. In the horizontal sections the number density of brine inclusions ranged from 0.95 to 1.08 per $\mathrm{mm}^{3}$. 
Light et al. (2003) also study the effect of temperature change on the structure of sea ice and note that both the horizontal and vertical dimensions of brine pockets were observed to increase. They become slightly more spherical upon cooling and slightly more elongated upon melting, though the overall aspect ratio changed little. The merging of inclusions was common at warmer temperatures, with the brine pocket density decreasing from 51 to 46 to 35 per $\mathrm{mm}^{3}$ as temperatures increased from $-15^{\circ} \mathrm{C}$ to $-5^{\circ} \mathrm{C}$ to $-2^{\circ} \mathrm{C}$. It should be noted that the brine pocket density in the warmed sample was larger than the overall averages seen. The authors label brine inclusions with a length smaller than $0.5 \mathrm{~mm}$ as pockets, while those with greater lengths are labelled as tubes. The behaviour of the brine tubes was slightly different from the pockets of brine, the diameter of the tube increased and decreased with temperature but the length remained nearly unchanged.

Pringle et al. (2009b) used x-ray computed tomography to image single sea ice crystals. The images show a change in pore space, as the temperature increases from $-18^{\circ} \mathrm{C}$ to $-8^{\circ} \mathrm{C}$ to $-4^{\circ} \mathrm{C}$ the porosity increases from $2.2 \%$ to $4.6 \%$ to $8.8 \%$. The structure observed in the images shows vertically elongated near parallel layers with 'necks' forming horizontally between them, as temperatures warm.

In addition to these observed temperature effects, the brine inclusions can migrate vertically, under the influence of changing thermal gradients, to regions of higher temperature (to the bottom of the ice cover in winter and the top in summer) (Mathews and Clarke, 1963). Because of diffusion the salinity of brine within an inclusion will be uniform. When in a thermal gradient one end of the inclusion will be warmer and thus the brine is too concentrated, leading to the ice dissolving to reduce its concentration. At the colder end the brine concentration is low and more ice freezes to increase its concentration. The net effect is to move the entire cell of brine along the thermal gradient (Pounder, 1965).

It has been suggested that the brine component of sea ice undergoes a percolation transition. The bulk properties of cold ice, containing a few small isolated brine inclusions, are dominated by the properties of the ice matrix. Upon warming the ice the brine pockets become increasingly interconnected over larger scales, and the bulk properties of sea ice become dominated by the properties of the brine component. Such a transition is common in many two phase systems. Within sea ice it has been proposed that a percolation transition in its fluid transport properties occurs at a brine volume 
fraction of about $5 \%$, which corresponds to a temperature of $-5^{\circ} \mathrm{C}$ for sea ice with a salinity of 5\% (Golden et al., 1998; 2007 and Golden 2003). For temperatures warmer than $-5^{\circ} \mathrm{C}$ brine carrying heat and nutrients can move through the ice, whereas for colder temperatures the ice is impermeable (Golden et al., 1998; 2007 and Golden 2003). 


\subsection{The electrical structure of sea ice}

The electrical resistivity of the pure ice matrix is much higher than that of the brine trapped within it (Ingham et al., 2008). For example the resistivity of pure ice is given by Mathews and Clark (1963) as $10^{7} \Omega \mathrm{m}$, while during our measurement periods the calculated brine resistivities (see section 3.9) are mostly below $1 \Omega \mathrm{m}$. Due to this significant difference measurements of the electrical resistivity structure of sea ice provide a means of investigating the brine microstructure and the evolution of the physical properties affected by this structure.

The bulk resistivity of sea ice depends on the porosity (or structure created by the brine pockets), the saturation of the pores with brine and the conductivity (or salinity) of the brine (Thyssen et al., 1974). In first-year sea ice, formed under quiescent conditions, the brine inclusions have a preferential vertical alignment. Therefore the bulk resistivity structure is anisotropic, with the resistivity in the vertical direction $\left(\rho_{v}\right)$ being lower than that in the horizontal direction $\left(\rho_{H}\right)$. In the presence of steady ocean currents it is possible that the c-axes of the ice crystals align parallel to the current in the horizontal plane (Kovacs and Morey, 1978; Nakawo and Sinha, 1984; Shokr and Sinha, 1994; Perovich and Gow, 1996; Eicken, 2003). Thus the horizontal component of the bulk resistivity may itself exhibit some anisotropy. However, in this thesis this is treated as typically insignificant compared to the anisotropy between the vertical and horizontal components of the bulk resistivity.

As temperature decreases brine inclusions shrink as more ice forms around the edges. The dissolved salts are rejected from this newly forming ice and therefore, the salinity of the remaining brine increases. Thus, initially, as the temperature decreases the brine salinity, and hence brine conductivity, increases. However, below about $-8^{\circ} \mathrm{C}$, brine conductivity decreases with decreasing temperature, even though the salinity is still increasing (Morey et al., 1984). At low temperatures ion mobility in the brine is reduced, therefore the brine conductivity begins to decrease with decreasing temperature (Haas et al., 1997). Thus, for sea ice conductivity the decreasing brine volume with decreasing temperature is compensated, up to a point, by increasing brine conductivity. As temperatures lower further and the brine conductivity begins to 
decrease, this and the decrease in brine volume combine to cause reduced ice conductivity.

Conversely, as sea ice warms ice melts from the brine inclusion walls, decreasing its salinity and hence brine conductivity. However, the melting ice also enlarges the brine inclusions with individual pores connecting and merging. Thus, despite the brine salinity (and brine conductivity) decreasing with increasing temperatures sea ice conductivity can increase due to the increasing number of conduction paths through the enlarging brine volume.

Many studies of the electrical properties of sea ice both in situ and on laboratory grown ice have been carried out.

Addison (1969) investigated the complex dielectric coefficient, resistivity and other related parameters of unidirectional artificially grown sea ice at temperatures of $-12.5^{\circ} \mathrm{C}$ to $-35^{\circ} \mathrm{C}$. Measurements were made over the frequency range $20 \mathrm{~Hz}$ to $100 \mathrm{MHz}$. From these experiments Addison (1969) observed a marked increase in resistivity with decreasing temperature, especially at the lower frequencies. Plots of resistivity vs. frequency, in Addison (1969), show that below $10 \mathrm{MHz}$ the resistivity decreases only slowly with increasing frequency.

Thyssen et al. (1974) carried out direct current (DC) resistivity soundings near Pond Inlet, N. W. T. during May and June 1972. Soundings were made at 22 sites along an approximate $1 \mathrm{~km}$ profile between Pond Inlet, Baffin Island and Bylot Island. Only young ice was found along the measurement profile. The soundings were repeated at intervals of approximately two weeks (16-19 May, 3-6 June, 15-21 June) to observe the resistivity variations with time. Measurements were made using a Schlumberger array (see section 2.1.3), with surface soundings used to obtain geometric mean resistivities $\left(\rho_{m}\right)$ and pit wall measurements used to obtain horizontal resistivities $\left(\rho_{H}\right)$.

Thyssen et al. (1974) derive a simplified three layer model for the sea ice, with a rise in apparent resistivity with depth then a rapid decrease due to the low resistivity of the underlying seawater. Measurements yielded values of $221 \Omega \mathrm{m}$ for the horizontal resistivity, $60 \Omega \mathrm{m}$ for the geometric mean and $16 \Omega \mathrm{m}$ for the vertical resistivity. These values gave a coefficient of anisotropy equal to $\lambda=0.26$ for unrafted ice. The authors also show that the apparent thickness of the ice, found from the resistivities, is equal to 
$\lambda$ times the true thickness (determined by drilling). The average resistivity was observed to decrease significantly during the observation period, as brine volume increased due to increasing temperature.

Timco (1979) analysed the same data set presented in Thyssen et al. (1974) in more detail, in terms of the microstructure of the sea ice. For each of the three measurement periods (16-19 May, 3-6 June, 15-21 June) their analysis yielded average vertical resistivities of $104 \Omega \mathrm{m}, 80 \Omega \mathrm{m}$ and $45 \Omega \mathrm{m}$ and average horizontal resistivities of $298 \Omega \mathrm{m}$, $230 \Omega \mathrm{m}$ and $129 \Omega \mathrm{m}$, with corresponding brine volume fractions of $40 \%$, $67 \%$ and $82 \%$. The author states that interpretation of the results indicated that the ice could be considered as a two-layer case; with a low resistivity layer for the upper $1 / 4$ of the ice sheet, and a higher resistivity layer for the lower $3 / 4$. Depending on the brine volume fraction the geometric mean resistivity of the upper layer varied between $30 \Omega \mathrm{m}$ to $112 \Omega \mathrm{m}$ while that of the lower layer ranged from $76 \Omega \mathrm{m}$ to $176 \Omega \mathrm{m}$.

Timco (1979) noted a decrease in resistivity with increasing temperature and brine volume fraction, which was attributed to changes in the size and geometry of the brine cells. Using average geometric mean and horizontal resistivities Timco (1979) came to a value of $\lambda=0.59$ for the coefficient of anisotropy of this ice. Timco (1979) used a mixing formula for the electrical resistivity of a two-phase heterogeneous system to arrive at a value of $1.7 \mathrm{~cm}$ for the "average" length of brine cells in relatively warm ice, with a brine volume fraction of between $40 \%$ and $70 \%$.

Morey et al. (1984) made in situ measurements of the electrical properties of sea ice during spring. From these measurements Morey et al. (1984) found; "(1) for sea ice with a preferred horizontal crystal c-axis alignment, the anisotropy of the ice increased with depth, (2) Brine inclusion conductivity increased with decreasing temperature down to about $-8^{\circ} \mathrm{C}$ below which the conductivity decreased with decreasing temperature, (3) The DC conductivity of sea ice increased linearly with increasing brine volume, (4) An exponential relationship was found between DC conductivity and temperature of sea ice”. Morey et al. (1984) also noted that at about $-8.2^{\circ} \mathrm{C}$ a phase change occurs in the brine and Mirabilite $\left(\mathrm{Na}_{2} \mathrm{SO}_{4} .10 \mathrm{H}_{2} \mathrm{O}\right)$ begins to precipitate out of solution. This dissolved salt is a contributor to the measured conductivity, and when it began to precipitate the authors observed an apparent effect on the rate of change in the DC conductivity. When the temperature reaches $-22.9^{\circ} \mathrm{C}$ Hydrohalite $\left(\mathrm{NaCl}_{2} 2 \mathrm{H}_{2} \mathrm{O}\right)$ 
begins to precipitate (Stogryn and Desargant, 1985). The effect of this lower temperature precipitation was observed by Addison (1969) with an extremely rapid change in resistivity at $-23^{\circ} \mathrm{C}$.

Buckley et al. (1986) measured the resistivity of first year sea ice at two locations in McMurdo Sound, Antarctica. The measurements were made using a Wenner array (see section 2.1.3) at audio frequencies $(100 \mathrm{~Hz}$ to $1000 \mathrm{~Hz})$. The authors note that the measured resistivities were insensitive to the applied frequency, as was observed by Addison (1969). In addition to resistivity, salinity and temperature profiles were also measured. From this research Buckley et al. (1986) found that the average resistivity of the sea ice lay within the range $50 \Omega \mathrm{m}$ to $200 \Omega \mathrm{m}$ and that the structure could be adequately described by a three layer model. This model was consistent with that obtained by Thyssen et al. (1974), being made up of a thin conducting surface layer, an insulating layer and finally seawater. Buckley et al. (1986) found that the resistivity of the surface layer was influenced by the removal of the snow cover. The depth of the ice, predicted by the Wenner sounding, was roughly $50 \%$ of the actual depth. Within the bulk layer of the ice this result is consistent with conductivity in the vertical direction being four times the conductivity in the horizontal direction, leading to a coefficient of anisotropy of $\lambda \sim 0.5$.

As the ice warmed through the spring of 2006 Ingham et al. (2008) carried out a series of DC cross-borehole measurements in first-year sea ice near Barrow, Alaska. The authors show that by positioning one current and one potential electrode in each of the boreholes and using specific electrode combinations a reasonable approximation of the horizontal resistivity can be obtained. Furthermore, assuming that the lowermost electrodes are in the underlying seawater, Ingham et al. (2008) note that the vertical alignment of electrodes is also able to accurately determine the boundary between the relatively resistive sea ice and the highly conducting seawater. This is seen in their resistivity models, with the seawater clearly resolved as an effective half space with a resistivity of $\sim 0.4 \Omega \mathrm{m}$.

By making resistivity measurements on three separate occasions as the ice warmed over the period April-June 2006, the authors were able to study the temporal evolution of the resistivity structure of the sea ice. From their models Ingham et al. (2008) show that through the bulk of the sea ice there is a general decrease in horizontal resistivity with 
increasing salinity and brine volume fraction. Furthermore, the authors suggested that the evolution of this resistivity structure showed that "(1) at temperatures less than $-5^{\circ} \mathrm{C}$ it is broadly consistent with the expected variation with brine volume fraction predicted by Archie's Law and (2) shows evidence of a percolation transition in the horizontal component of the resistivity when brine volume fractions exceed $8 \%-10 \%$ ".

A halo of anomalous resistivity structure around the electrode strings was observed in the models produced from this study. It was suggested that after insertion of the electrode strings, as the ice reformed in the boreholes and subsequently thickened, the microstructure in the immediate vicinity of the strings was significantly affected by their presence. The authors reasoned that the existence of the halo around the electrode strings provided a strong argument as to why it is preferable to use cross-borehole measurements to determine $\rho_{H}$ rather than single borehole measurements. 


\section{Chapter 2 Electrical resistivity surveys}

\subsection{Resistivity survey principles}

The purpose of geophysical electrical surveys is to investigate the subsurface of a medium with a varied resistivity structure. These surveys are thus ideal for determining the internal structure of sea ice, with a large contrast between the resistivities of the brine and solid ice components. The resistivity of the medium is related to various parameters such as the mineral and fluid content, porosity and degree of fluid saturation. Electrical resistivity surveys have been used for many decades in hydrogeological, mining and geotechnical investigations and more recently environmental surveys (Loke, 2004). Since measurements are generally made either at the surface of the medium or through undisturbed material between boreholes, this technique complies with the in situ constraint, necessary to obtain an accurate physical image of the medium in its natural state.

\subsubsection{Resistivity of an isotropic medium}

The fundamental physical law used in resistivity surveys is Ohm's Law ( $\underline{J}=\sigma \underline{E}=-\nabla V / \rho)$, which governs the flow of current in a medium (Loke, 2004). In an isotropic medium Ohm's law and the equation of continuity $(\nabla . \underline{J}=0)$ can be combined to give

$$
\nabla \cdot(\nabla V / \rho)=0
$$

Here $J$ is the electric current density $\left(\mathrm{A} / \mathrm{m}^{2}\right), \sigma$ is conductivity $\left(\mathrm{Sm}^{-1}\right)$ which is defined as the reciprocal of resistivity ( $\rho$, units $\Omega \mathrm{m}), E$ the electric field $(\mathrm{V} / \mathrm{m}), V$ the electric potential (V) and $W$ the potential gradient $(\mathrm{V} / \mathrm{m})$.

If the medium is homogeneous $\rho$ (resistivity) is independent of the coordinate axes and the above equation reduces to

$$
\left(\nabla^{2} V\right) / \rho=0 \quad \Rightarrow \quad\left(\nabla^{2} V\right)=0
$$


Thus, in a homogeneous isotropic medium, the electric potential satisfies Laplace's equation.

Suppose a current $I$ is injected through an electrode at the surface of a semi infinite homogeneous medium. The potential at some distance $(r)$ from the introduced current will only depend on that distance. Noting this, Laplace's equation, from above, can be expanded in spherical coordinates to give

$$
\nabla^{2} V=\frac{1}{r^{2}} \frac{\partial}{\partial r}\left(r^{2} \frac{\partial V}{\partial r}\right)=\frac{2}{r} \frac{\partial V}{\partial r}+\frac{\partial^{2} V}{\partial r^{2}}=0
$$

A solution of which is

$$
V=C_{1}+\frac{C_{2}}{r}
$$

where $C_{1}$ and $C_{2}$ are constants

As $V$ must be zero at large $r, C_{1}=0$ and

$$
V=\frac{C_{2}}{r}
$$

Using equation (2.1), Ohm's law given earlier, and the fact that $W$ is only dependent on $r$, the current density can be found

$$
\underline{J}=\frac{-\nabla V}{\rho}=\frac{-1}{\rho}\left(\frac{\partial V}{\partial r} \underline{i}+\frac{1}{r} \frac{\partial V}{\partial \theta} \underline{j}+\frac{1}{r \sin \theta} \frac{\partial V}{\partial \phi} \underline{k}\right) \Rightarrow J=\frac{-1}{\rho} \frac{\partial V}{\partial r}=\frac{1}{\rho} \frac{C_{2}}{r^{2}}
$$

where $\theta$ is the angle from vertical to the vector $r$ (the line between where the current is injected and the potential measured) and $\phi$ is the horizontal angle from the x coordinate axis to the horizontal projection of the vector $r$.

The current $I$ can be obtained by multiplying the current density $(J)$ by the area $(A)$ through which the current travels. For surface electrodes this area is the surface area of a hemisphere $\left(2 \pi r^{2}\right)$, while for buried electrodes this area becomes the surface area of a sphere $\left(4 \pi r^{2}\right)$

$$
I=A \times J=2 \pi r^{2} \frac{1}{\rho} \frac{C_{2}}{r^{2}}=\frac{2 \pi C_{2}}{\rho}
$$

Rearranging gives

$$
C_{2}=I \rho / 2 \pi
$$


which, when substituted into equation (2.1) above gives the potential at a distance $r$ from where the current is introduced at the surface of a semi infinite homogeneous medium.

$$
V=I \rho / 2 \pi r
$$

Alternatively, for a buried electrode the potential is given by

$$
V=I \rho / 4 \pi r
$$

The majority of electrical imaging surveys make use of four electrodes. Two electrodes are used to introduce current into the medium, one being a current source $(C l)$ the other a sink $(C 2)$. The potential difference is then measured between two more electrodes $(P 1$ and $P 2$ ) and can be expressed as

$$
\Delta V=\left(V_{11}+V_{12}\right)-\left(V_{21}+V_{22}\right)
$$

where $V_{i j}$ is the potential measured at potential electrode ' $i$ ' due to current electrode ' $j$ ', and terms $V_{12}$ and $V_{22}$ will be negative as $C 2$ is a current sink.

\subsubsection{Resistivity of an anisotropic medium}

In first-year sea ice the preferential vertical alignment of the brine inclusions means that the bulk resistivity structure of the ice is anisotropic, with the vertical component of the bulk resistivity lower than the horizontal component.

In an anisotropic medium, as shown by Bhattacharya and Patra (1968), Ohm's law $(\underline{J}=\sigma \underline{E})$ can be modified to

$$
\begin{aligned}
& J_{x}=\sigma_{x x} E_{x}+\sigma_{x y} E_{y}+\sigma_{x z} E_{z} \\
& J_{y}=\sigma_{y x} E_{x}+\sigma_{y y} E_{y}+\sigma_{y z} E_{z} \\
& J_{z}=\sigma_{z x} E_{x}+\sigma_{z y} E_{y}+\sigma_{z z} E_{z}
\end{aligned}
$$

It can be shown that $\sigma_{i k}=\sigma_{k i}$, and that it is always possible to orientate the coordinate axes such that $\sigma_{x y}=\sigma_{y z}=\sigma_{z x}=0$. These axes are then called the principle axes of anisotropy. When the measurement axes are orientated parallel to the principle axes the equations above reduce to

$$
\begin{aligned}
& J_{x}=\sigma_{x x} E_{x}=E_{x} / \rho_{x}=-1 / \rho_{x}(\partial V / \partial x) \\
& J_{y}=\sigma_{y y} E_{y}=E_{y} / \rho_{y}=-1 / \rho_{y}(\partial V / \partial y) \\
& J_{z}=\sigma_{z z} E_{z}=E_{z} / \rho_{z}=-1 / \rho_{z}(\partial V / \partial z)
\end{aligned}
$$


The equation of continuity $(\nabla . \underline{J}=0)$ thus becomes

$$
\frac{\partial}{\partial x}\left(\frac{1}{\rho_{x}} \frac{\partial V}{\partial x}\right)+\frac{\partial}{\partial y}\left(\frac{1}{\rho_{y}} \frac{\partial V}{\partial y}\right)+\frac{\partial}{\partial z}\left(\frac{1}{\rho_{z}} \frac{\partial V}{\partial z}\right)=0
$$

If the anisotropic medium is homogeneous, $\rho$ is independent of the coordinate axes and the equation above becomes

$$
\frac{1}{\rho_{x}}\left(\frac{\partial^{2} V}{\partial x^{2}}\right)+\frac{1}{\rho_{y}}\left(\frac{\partial^{2} V}{\partial y^{2}}\right)+\frac{1}{\rho_{z}}\left(\frac{\partial^{2} V}{\partial z^{2}}\right)=0
$$

By redefining the coordinate system to allow, $\alpha=x \sqrt{\rho_{x}}, \beta=y \sqrt{\rho_{y}}$ and $\eta=z \sqrt{\rho_{z}}$ this equation then reduces to Laplace's equation

$$
\left(\frac{\partial^{2} V}{\partial \alpha^{2}}\right)+\left(\frac{\partial^{2} V}{\partial \beta^{2}}\right)+\left(\frac{\partial^{2} V}{\partial \eta^{2}}\right)=0
$$

which has a solution

$$
V=\frac{C}{\left(\alpha^{2}+\beta^{2}+\eta^{2}\right)^{1 / 2}}=\frac{C}{\left(x^{2} \rho_{x}+y^{2} \rho_{y}+z^{2} \rho_{z}\right)^{1 / 2}}
$$

where $C$ is a constant, $x, y$ and $z$ are the distances between where the current is injected and the potential is measured, along the respective axes and $\rho_{i}$ is the resistivity in the $i$ direction

As most of the brine inclusions within the sea ice are elongated vertically the resistivity structure of the ice can be approximated by a resistivity structure that is the same in the two horizontal directions $\left(\rho_{x}=\rho_{y}=\rho_{H}\right)$ but different in the vertical direction $\left(\rho_{z}=\rho_{V}\right)$. Although it is possible that in the presence of steady ocean currents the horizontal resistivity itself may exhibit some anisotropy, (Kovacs and Morey, 1978; Nakawo and Sinha, 1984; Shokr and Sinha, 1994; Perovich and Gow, 1996; Eicken, 2003), in the present analysis we treat this as typically insignificant compared to the anisotropy between the vertical and horizontal resistivity. This assumption is supported by the closely matched resistivity values observed from surface soundings running approximately parallel and perpendicular to the coast at Barrow, Alaska.

In the case of isotropic horizontal resistivity (see Appendix I for treatment of anisotropic horizontal resistivity) equation (2.5) becomes 


$$
V=\frac{C}{\left(x^{2} \rho_{H}+y^{2} \rho_{H}+z^{2} \rho_{V}\right)^{1 / 2}}
$$

where $\rho_{H}$ is the horizontal component of the bulk resistivity and $\rho_{V}$ the vertical component.

The coefficient of anisotropy $(\lambda)$ and the geometric mean resistivity $\left(\rho_{m}\right)$ are defined as

$$
\begin{aligned}
& \lambda=\sqrt{\rho_{V} / \rho_{H}} \\
& \rho_{m}=\sqrt{\rho_{V} \rho_{H}}
\end{aligned}
$$

Since the brine inclusions have a lower resistivity than the solid ice matrix and are preferentially elongated vertically, the vertical component of the bulk resistivity will be smaller than the horizontal component and the coefficient of anisotropy of sea ice will be less than one.

Using expressions (2.6) and (2.7) the equation above, for potential, can be simplified to

$$
V=\frac{C}{\rho_{H}^{1 / 2}\left(x^{2}+y^{2}+\lambda^{2} z^{2}\right)^{1 / 2}}
$$

Thus using equation (2.8) the current densities given in equations (2.4) become

$$
\begin{aligned}
& J_{x}=\frac{x C}{\rho_{H}{ }^{3 / 2}\left(x^{2}+y^{2}+\lambda^{2} z^{2}\right)^{3 / 2}} \\
& J_{y}=\frac{y C}{\rho_{H}{ }^{3 / 2}\left(x^{2}+y^{2}+\lambda^{2} z^{2}\right)^{3 / 2}} \\
& J_{z}=\frac{z C}{\rho_{H}{ }^{3 / 2}\left(x^{2}+y^{2}+\lambda^{2} z^{2}\right)^{3 / 2}}
\end{aligned}
$$

with the total current density given by

$$
J=\left(J_{x}{ }^{2}+J_{y}{ }^{2}+J_{z}{ }^{2}\right)^{1 / 2}=\frac{\left(x^{2}+y^{2}+z^{2}\right)^{1 / 2} C}{\rho_{H}{ }^{3 / 2}\left(x^{2}+y^{2}+\lambda^{2} z^{2}\right)^{3 / 2}}
$$

Changing equation (2.8) and (2.9) to be in spherical coordinates gives

$$
\begin{aligned}
& V=\frac{C}{\rho_{H}{ }^{1 / 2} r\left(\sin ^{2} \theta+\lambda^{2} \cos ^{2} \theta\right)^{1 / 2}} \\
& J=\frac{C}{\rho_{H}{ }^{3 / 2} r^{2}\left(\sin ^{2} \theta+\lambda^{2} \cos ^{2} \theta\right)^{3 / 2}}
\end{aligned}
$$


where $C$ is a constant, $\rho_{H}$ is the horizontal component of the bulk resistivity, $\lambda$ is the coefficient of anisotropy, $r$ is the distance between where the current is injected and the potential measured and $\theta$ the angle from the vertical to the vector $r$.

\subsubsection{Surface electrical resistivity surveys}

There are many different electrode configurations. However, commonly used surface arrangements include the Wenner and Schlumberger arrays shown in Figure 2.1 below. The Schlumberger array is one of the most commonly used arrays for resistivity sounding surveys. The Wenner array is typically used in hydrological studies where the bulk resistivity is generally considered isotropic.

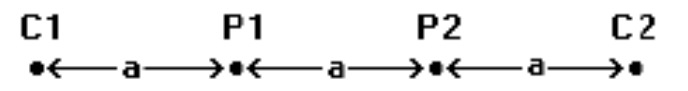

(a)

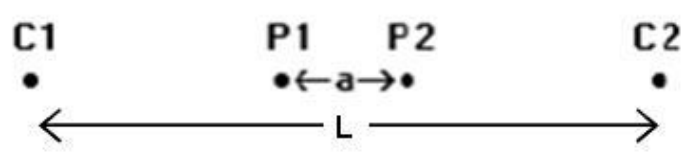

(b)

Figure 2.1: Commonly used electrode configurations for surface surveys (a) The Wenner array and (b) The Schlumberger array.

For the Wenner array the electrodes are equally spaced on the surface of the medium with the two potential electrodes in the centre and the two current electrodes on the outside. The Schlumberger array is similar, but with the distance between the current electrodes designated by $L$ and not in general equal to $3 a$. As the distance ( $3 a$ or $L$ ) between the current electrodes is increased more current passes deeper into the ground and data on the resistivity structure with depth is obtained.

These examples are forms of dipole - dipole arrays, which use all four electrodes during measurement. Other electrical imaging surveys make use of only three or two electrodes during measurement, in practice these are only approximations of three and two electrode arrays as four electrodes are still positioned in the field. For example, pole dipole arrays uses three electrodes, with the fourth as a remote electrode (usually either $C 2$ or $P 2$ ) which must be placed at a distance of at least 20 times the maximum separation between $C 1$ and $P 1$. The ideal pole-pole array, with only one current and one potential electrode, is approximated by using the second current and potential electrodes ( $C 2$ and $P 2$ ) as remote electrodes, which must be placed sufficiently far from the survey 
line. A disadvantage of these arrays is that with electrode P2 as a remote electrode the large distance between the P1 and P2 electrodes means that a large amount of telluric noise can be picked up, and the quality of the measurements severely degraded.

\subsubsection{Measurements on a semi-infinite uniform anisotropic medium}

Current from an electrode placed at the surface of an anisotropic medium, such as sea ice, must pass through a hemisphere of radius $r$ centred on the electrode. The total current flowing out through this surface (and thus the current at the electrode) is given by

$$
I=\int_{s} J \cdot d s=\int_{0}^{2 \pi} \int_{\pi / 2}^{\pi} J r^{2} \sin \theta d \theta d \phi
$$

Inserting equation (2.11) into equation (2.12) and solving and simplifying gives $I=\frac{2 \pi C}{\rho_{H}{ }^{3 / 2} \lambda}$, which rearranges to $C=\frac{\rho_{H}{ }^{3 / 2} \lambda I}{2 \pi}$

Substituting $\mathrm{C}$ into equation (2.10) gives

$$
V=\frac{\rho_{m} I}{2 \pi r\left(1+\left(\lambda^{2}-1\right) \cos ^{2} \theta\right)^{1 / 2}}
$$

and converting back into Cartesian coordinates leads to

$$
V=\frac{\rho_{m} I}{2 \pi\left(r^{2}+z^{2} \lambda^{2}-z^{2}\right)^{1 / 2}}
$$

where $r$, the distance between where the current is injected and where the potential is measured, is given by $r=\left(x^{2}+y^{2}+z^{2}\right)^{1 / 2}$. The $x, y$ and $z$ are the distances, between where the current is injected and the potential measured, as measured along the relevant axis.

For a common surface array (see Figure 2.1 above), the potential $\left(V_{i j}\right)$ measured at potential electrode ' $i$ ' at $\left(x_{i}, y_{i}, z_{i}\right)$ due to current electrode ' $j$ ' at $\left(x_{j}, y_{j}, z_{j}\right)$ can be obtained from equation (2.13) with $\theta=90^{\circ}$ or equation (2.14) with $z=\left|z_{i}-z_{j}\right|=0$. The distance between the two electrodes is given by $r_{i j}=\sqrt{\left|x_{i}-x_{j}\right|^{2}+\left|y_{i}-y_{j}\right|^{2}}$ hence $\Rightarrow$ $V_{i j}=\frac{\rho_{m} I}{2 \pi r_{i j}}$

Substituting $V_{i j}$ into equation (2.3) for the potential difference gives 


$$
\Delta V=\frac{\rho_{m} I}{2 \pi}\left(\frac{1}{r_{11}}-\frac{1}{r_{12}}-\frac{1}{r_{21}}+\frac{1}{r_{22}}\right) \Rightarrow \Delta V=\frac{\rho_{m} I}{2 \pi} g
$$

where the terms arising from $V_{12}$ and $V_{22}$ are negative as $C 2$ is a current sink, and $g$ is the geometric factor, determined by the spacing of the electrodes $\left(r_{i j}\right)$.

This equation gives the potential difference that would be measured over a homogenous anisotropic half space. Actual field surveys are invariably conducted over an inhomogeneous medium and the recorded $\Delta V$ and current values are used to calculate an apparent resistivity $\rho_{a}$ (defined as the average resistivity value of an equivalent halfspace)

$$
\rho_{a}=2 \pi \frac{\Delta V}{I}\left[\frac{1}{g}\right]
$$

From equation (2.15) it can be seen that surface resistivity readings are only sensitive to the geometric mean resistivity of the medium $\left(\rho_{m}\right)$. Previous studies on sea ice (Thyssen et al., 1974; Buckley et al., 1986 and Ingham et al., 2008) have also indicated that for an anisotropic resistivity structure surface soundings underestimate the true thickness of the medium.

\subsubsection{Borehole resistivity measurements}

Surface electrical resistivity soundings can be limited by a reduction in resolution with depth and the fact that on an anisotropic medium such as sea ice, they are only sensitive to the geometric mean of the bulk resistivity structure. In theory the only way to improve the resolution at depth is to place the electrodes closer to the structures of interest. Thus surveys carried out with electrodes positioned in boreholes can give more accurate results than is possible with surface surveys alone. Furthermore, as was indicated by measurements made by Thyssen et al. (1974), in the vertical wall of a pit in sea ice, and as will be seen in the theory outlined below, using borehole measurements will enable both the geometric mean and the horizontal components of the bulk resistivity structure to be obtained.

As the medium is disturbed when the boreholes are drilled, it is desirable to make crossborehole measurements (i.e. both current and both potential electrodes cannot be in the same borehole) to meet the in situ constraints. Making measurements between 
boreholes forces the current to pass through undisturbed intact material. Thus crossborehole measurements allow investigation of the sea ice in its natural state.

As with the surface surveys, borehole surveys can be implemented with a variety of electrode and borehole configurations. Individual measurements can make use of two, three or four electrodes, but also two, three or four boreholes. For an approximation of a two electrode array one current and one potential electrode are positioned in two separate boreholes. The remaining potential and current electrodes are used as remote electrodes and must be positioned sufficiently far away (a distance of a least 20 times the maximum separation of $C 1$ and $P 1$ ) so that the pole-pole approximation is accurate. Both the electrodes could be positioned in the same borehole, however the array would only be sensitive to changes in resistivity in the vicinity of the borehole. Furthermore, as mentioned earlier, a large distance between the potential electrodes leads to the problem of contamination by telluric noise. For an approximation of a three electrode array there are several different combinations that can be used, though these are somewhat limited if we want to examine the undisturbed structure between boreholes and thus cannot have both the current or both the potential electrodes in the same borehole. Options include positioning $C 1$ and $P 1, C 1$ and $P 2, C 2$ and $P 1$ or $C 2$ and $P 2$ in the same borehole with the third electrode in a second borehole and the fourth electrode as a remote. Further to these arrangements three electrodes can be in three individual boreholes with the remote electrode adequately far from the survey area. When four electrodes are used there are several options: two boreholes with one current and one potential electrode in each, three boreholes with a current and potential electrode in one and the remaining current and potential electrode in separate boreholes, or four boreholes each containing its own electrode. (Loke, M. H., 2004)

\subsubsection{Measurements in a infinite uniform anisotropic medium}

For an electrode buried in an anisotropic medium, such as sea ice, the current must pass through a sphere of radius $r$ centred on the electrode. In this case Bhattacharya and Patra (1968) show that the current at the electrode is given by

$$
I=\int_{s} J \cdot d s=\int_{0}^{2 \pi} \int_{0}^{\pi} J r^{2} \sin \theta d \theta d \phi
$$

Inserting equation (2.11) from section 2.1.2 into equation (2.16) and solving and simplifying gives $I=\frac{4 \pi C}{\rho_{H}{ }^{3 / 2} \lambda}$ which rearranges to $C=\frac{\rho_{H}{ }^{3 / 2} \lambda I}{4 \pi}$ 
Substituting $C$ into equation (2.10) from section 2.1.2 gives

$$
V=\frac{\rho_{m} I}{4 \pi r\left(1+\left(\lambda^{2}-1\right) \cos ^{2} \theta\right)^{1 / 2}}
$$

This is the same as the expression obtained for the surface soundings (see equation (2.13)) except the geometric $2 \pi$ term has become $4 \pi$, due to the change from a hemispheroid surface of current to a spheroid surface when the electrode is buried.

Converting equation (2.17) into Cartesian coordinates leads to

$$
V=\frac{\rho_{m} I}{4 \pi\left(r^{2}+z^{2} \lambda^{2}-z^{2}\right)^{1 / 2}}
$$

where $r$, being the distance between where the current is injected and where the potential is measured, is given by $r=\left(x^{2}+y^{2}+z^{2}\right)^{1 / 2}$. Thus

$$
V=\frac{\rho_{m} I}{4 \pi\left(x^{2}+y^{2}+z^{2} \lambda^{2}\right)^{1 / 2}}
$$

where $x, y$ and $z$ are the distances, between where the current is injected and the potential measured, as measured along the relative axis. 


\subsection{Cross-borehole resistivity measurements in a infinite anisotropic medium}

Considering a general arrangement of four electrodes in four separate boreholes (as in Figure 2.2) expression (2.18) above can be used to give the potential $\left(V_{i j}\right)$ measured at a buried potential electrode ' $i$ ' at $\left(x_{i}, y_{i}, z_{i}\right)$ due to a buried current electrode ' $j$ ' at $\left(x_{j}, y_{j}\right.$, $z_{j}$ ), giving

$$
V_{i j}=\frac{I \rho_{m}}{4 \pi}\left(\frac{1}{\sqrt{\left|x_{i}-x_{j}\right|^{2}+\left|y_{i}-y_{j}\right|^{2}+\lambda^{2}\left|z_{i}-z_{j}\right|^{2}}}\right)=\frac{I \rho_{m}}{4 \pi} \frac{1}{r_{i j}} T_{i j}
$$

where $T_{i j}=\left(\frac{\sqrt{\left|x_{i}-x_{j}\right|^{2}+\left|y_{i}-y_{j}\right|^{2}+\left|z_{i}-z_{j}\right|^{2}}}{\sqrt{\left|x_{i}-x_{j}\right|^{2}+\left|y_{i}-y_{j}\right|^{2}+\lambda^{2}\left|z_{i}-z_{j}\right|^{2}}}\right)=\left(\frac{r_{i j}}{\sqrt{\left|x_{i}-x_{j}\right|^{2}+\left|y_{i}-y_{j}\right|^{2}+\lambda^{2}\left|z_{i}-z_{j}\right|^{2}}}\right)$

- $x=\left|x_{i}-x_{j}\right|$

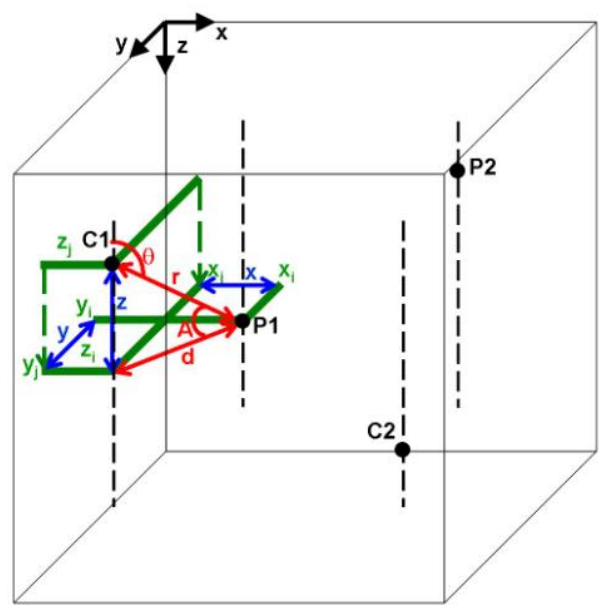

- $y=\left|y_{i}-y_{j}\right|$

- $z=\left|z_{i}-z_{j}\right|$

- $d=\sqrt{x^{2}+y^{2}}$ $d=d_{i j}=\sqrt{\left|x_{i}-x_{j}\right|^{2}+\left|y_{i}-y_{j}\right|^{2}}$

- $r=\sqrt{z^{2}+d^{2}}$ $r=r_{i j}=\sqrt{\left|x_{i}-x_{j}\right|^{2}+\left|y_{i}-y_{j}\right|^{2}+\left|z_{i}-z_{j}\right|^{2}}$

- $\sin (A)=\frac{z}{r}$

- $\theta=A+\frac{\pi}{2}$

Figure 2.2: Electrode configuration, with two current and two potential electrodes positioned individually in four boreholes within the ice.

The potential difference between electrodes $P 1$ and $P 2$ can then be found using (2.19) and (2.3) to give

$$
\Delta V=\frac{I \rho_{m}}{4 \pi}\left(\frac{T_{11}}{r_{11}}-\frac{T_{12}}{r_{12}}-\frac{T_{21}}{r_{21}}+\frac{T_{22}}{r_{22}}\right)
$$

where $T_{i j}$ is defined as in equation (2.19) and $r_{i j}$ is the distance between electrodes. 
If measurements were made in a medium of uniform isotropic resistivity, $\rho$, then an expression for the potential can be obtained from (2.2b) and the potential difference from (2.3), leading to

$$
\Delta V=\frac{I \rho}{4 \pi}\left(\frac{1}{r_{11}}-\frac{1}{r_{12}}-\frac{1}{r_{21}}+\frac{1}{r_{22}}\right)
$$

Determining the conditions under which equation (2.20) reduces to equation (2.21), when $\rho$ is considered to be $\rho_{V}, \rho_{H}$ or $\rho_{m}$, shows which electrode combinations, if any, it is possible to use to yield a determination of the relevant resistivity.

\subsubsection{Measurements of horizontal resistivity $\left(\rho_{H}\right)$}

The measurement of the horizontal component of the anisotropic resistivity has previously been discussed by Ingham et al. (2008), and their arguments are mentioned here. Equation (2.20) can be rewritten using equations (2.6) and (2.7) to give

$$
\Delta V=\frac{I \rho_{H} \lambda}{4 \pi}\left(\frac{T_{11}}{r_{11}}-\frac{T_{12}}{r_{12}}-\frac{T_{21}}{r_{21}}+\frac{T_{22}}{r_{22}}\right)
$$

In this form (2.20) reduces to equation (2.21), with $\rho$ set to $\rho_{H}$, if all the $T_{i j}$ 's equal $1 / \lambda$. This occurs if $\left|x_{i}-x_{j}\right|^{2}+\left|y_{i}-y_{j}\right|^{2}=0$ or at least $\left|z_{i}-z_{j}\right|>>\sqrt{\left|x_{i}-x_{j}\right|^{2}+\left|y_{i}-y_{j}\right|^{2}}$. This first condition implies that all four electrodes must be in a single borehole. Timco (1979) effectively met this condition by using four electrodes aligned vertically in the side of an ice pit to measure $\rho_{H}$. However, in the case of four electrodes within the same borehole the formation of an anomalous halo around the electrode string when it is frozen into the ice raises the likelihood that measurements will be significantly affected by ice that is not in its natural state. This option is therefore excluded. The second condition is met if the current electrode and potential electrode are much more widely separated vertically than they are horizontally. This condition will have practical limitations in a real situation in which the anisotropic structure under study is relatively thin. As this is the case for first-year sea ice, for which typical thicknesses are $\sim 2 \mathrm{~m}$, this possibility is not considered.

An additional possibility, as described by Ingham et al. (2008), is to consider only two boreholes each containing one current and one potential electrode, such that $C l$ is at $\left(\mathrm{x}_{1}\right.$, 
$\left.\mathrm{y}_{1}, \mathrm{z}_{1}\right), C 2$ is at $\left(\mathrm{x}_{2}, \mathrm{y}_{2}, \mathrm{z}_{2}\right), P 1$ is at $\left(\mathrm{x}_{1}, \mathrm{y}_{1}, \mathrm{z}_{3}\right)$ and $P 2$ is at $\left(\mathrm{x}_{2}, \mathrm{y}_{2}, \mathrm{z}_{4}\right)$, as in Figure 2.3 below.

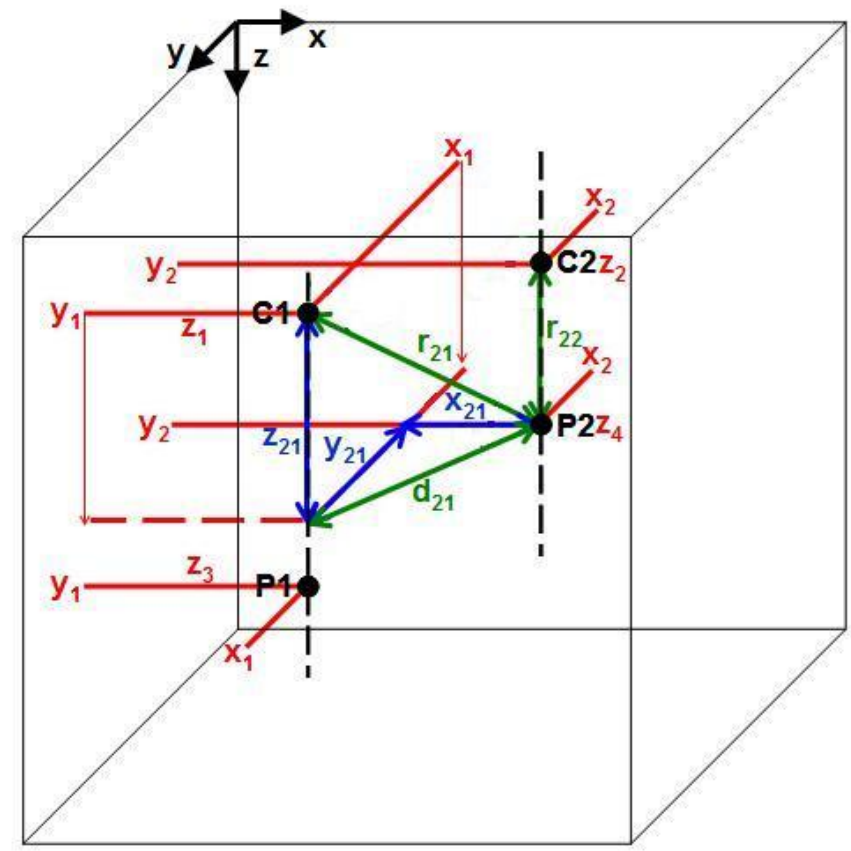

Figure 2.3: Electrode configuration, with two boreholes each containing one current and one potential electrode.

In this situation, in an anisotropic medium, the potential at $P 2$ due to $C 2$ and the potential at $P l$ due to $C l$ can be obtained from equation (2.19) with $r_{i j}=\left|z_{i}-z_{j}\right|$, $\left|x_{i}-x_{j}\right|=0$ and $\left|y_{i}-y_{j}\right|=0$ giving

$$
V_{11}=\frac{I \rho_{m}}{4 \pi} \frac{1}{\lambda\left|z_{3}-z_{1}\right|}=\frac{I \rho_{m}}{4 \pi} \frac{1}{\lambda r_{11}} \text { and } V_{22}=\frac{-I \rho_{m}}{4 \pi} \frac{1}{\lambda\left|z_{4}-z_{2}\right|}=\frac{-I \rho_{m}}{4 \pi} \frac{1}{\lambda r_{22}}
$$

Similarly the potential at $P 2$ due to $C 1$ and the potential at $P 1$ due to $C 2$ can be obtained from equation (2.19) with $r_{i j}=\sqrt{\left|x_{i}-x_{j}\right|^{2}+\left|y_{i}-y_{j}\right|^{2}+\left|z_{i}-z_{j}\right|^{2}}$ giving

$$
V_{12}=\frac{-I \rho_{m}}{4 \pi}\left(\frac{1}{\sqrt{\left|x_{1}-x_{2}\right|^{2}+\left|y_{1}-y_{2}\right|^{2}+\lambda^{2}\left|z_{3}-z_{2}\right|^{2}}}\right)=\frac{-I \rho_{m}}{4 \pi} \frac{T_{12}}{r_{12}}
$$

and

$$
V_{21}=\frac{I \rho_{m}}{4 \pi}\left(\frac{1}{\sqrt{\left|x_{2}-x_{1}\right|^{2}+\left|y_{2}-y_{1}\right|^{2}+\lambda^{2}\left|z_{4}-z_{1}\right|^{2}}}\right)=\frac{I \rho_{m}}{4 \pi} \frac{T_{21}}{r_{21}}
$$


Thus using equation (2.3) the potential difference between electrodes $P 1$ and $P 2$ is given by

$$
\begin{aligned}
\Delta V & =\frac{I \rho_{m}}{4 \pi}\left(\frac{1}{\lambda r_{11}}-\frac{T_{12}}{r_{12}}-\frac{T_{21}}{r_{21}}+\frac{1}{\lambda r_{22}}\right) \\
& =\frac{I \rho_{H}}{4 \pi}\left(\frac{1}{r_{11}}-\frac{\lambda T_{12}}{r_{12}}-\frac{\lambda T_{21}}{r_{21}}+\frac{1}{r_{22}}\right)
\end{aligned}
$$

In an isotropic medium of resistivity $\rho_{H}$ the potential difference would be given by expression (2.21) with:

$\rho=\rho_{H}, r_{11}=\left|z_{3}-z_{1}\right|, r_{22}=\left|z_{4}-z_{2}\right|, r_{12}=\sqrt{\left|x_{1}-x_{2}\right|^{2}+\left|y_{1}-y_{2}\right|^{2}+\left|z_{3}-z_{2}\right|^{2}}$ and $r_{21}=\sqrt{\left|x_{2}-x_{1}\right|^{2}+\left|y_{2}-y_{1}\right|^{2}+\left|z_{4}-z_{2}\right|^{2}}$

Consider a situation where the horizontal separation of the boreholes is a multiple $(n)$ of the vertical separation of the electrodes i.e. $\sqrt{\left|x_{i}-x_{j}\right|^{2}+\left|y_{i}-y_{j}\right|^{2}}=n\left|z_{i}-z_{j}\right|$. For an isotropic medium the ratio of the $V_{11}$ and $V_{22}$ terms to the $V_{12}$ and $V_{21}$ terms is given by $1: 1 / \sqrt{n^{2}+1}$. In this study the boreholes are located on the corners of a $1 \mathrm{~m}$ square, i.e. $x_{i}-x_{j}$ and $y_{i}-y_{j}$ are equal to zero or one, and the vertical electrode separation is between $0.1 \mathrm{~m}$ to $0.4 \mathrm{~m}$. Under these conditions the value of $n$ is between 2.5 and 15 , and the $V_{12}$ and $V_{21}$ terms are approximately between a $1 / 3$ and a $1 / 15$ the size of the $V_{11}$ and $V_{22}$ terms. Thus the $V_{12}$ and $V_{21}$ terms can be considered insignificant and can be ignored, giving the following expression for the potential difference measured in an isotropic medium of resistivity $\rho_{H}$

$$
\Delta V=\frac{I \rho_{H}}{4 \pi}\left(\frac{1}{r_{11}}+\frac{1}{r_{22}}\right)
$$

Ignoring these smaller terms in the equation gives at most an error of $\sim 30 \%$ in the spatial term.

Considering this same situation in an anisotropic medium the ratio of the $V_{11}$ and $V_{22}$ terms to the $V_{12}$ and $V_{21}$ terms is given by $1: 1 / \sqrt{\left(n^{2} / \lambda^{2}\right)+1}$. With the value of $n$ being between 2.5 and 15, and the typical values for the coefficient of anisotropy of sea ice being $\lambda \leq 0.6$ (see previous studies by Thyssen et al., 1974, Buckley et al., 1986 and Ingham et al., 2008), the $V_{12}$ and $V_{21}$ terms are, at most, approximately between a $1 / 4$ and a $1 / 25$ the size of the $V_{11}$ and $V_{22}$ terms. Thus the $V_{12}$ and $V_{21}$ terms can be 
considered insignificant and can be ignored, giving the following expression for the potential difference measured in an anisotropic medium.

$$
\Delta V=\frac{I \rho_{H}}{4 \pi}\left(\frac{1}{r_{11}}+\frac{1}{r_{22}}\right)
$$

Ignoring these smaller terms in the equation gives at most an error of $\sim 25 \%$ in the spatial term.

The expression for the potential difference above is the same as that obtained in an isotropic medium of resistivity $\rho=\rho_{H}$. Hence, with $\lambda \leq 0.6$ and the appropriate electrode combinations being used, giving $n$ between 2.5 and 15, measurements made between two boreholes allows reasonable values of $\rho_{H}$ to be estimated for an infinite uniform anisotropic medium. It should be noted that the larger the value of $n$ the greater the difference in size between the small vertical spacing between the electrodes and the larger horizontal spacing between boreholes. Furthermore, larger $n$ values leads to larger differences between the $V_{11}, V_{22}$ and the $V_{12}, V_{21}$ terms, giving greater validation to the removal of the smaller terms, and thus a better approximation of the potential difference.

Since we can ignore potentials $V_{12}$ and $V_{21}$, in the above potential difference expressions, we are left with $V_{11}$ and $V_{22}$ which are potentials measured at $P 1$ due to $C 1$ and $P 2$ due to $C 2$. As $P 1, C 1$ and $P 2, C 2$ are in the same boreholes it would seem that measurements are being made only along the borehole and not through the undisturbed ice between the boreholes. However as the current is flowing from source $C 1$ to sink $C 2$ (which are in different boreholes) it is passing through the undisturbed ice and will be affected by this structure. Thus the current reaching $P 1$ (from $C 1$ ) will be dependent on the undisturbed structure between the boreholes.

\subsubsection{Measurements of geometric mean resistivity $\left(\rho_{m}\right)$}

Equation (2.20) will reduce to the form of equation (2.21), with $\rho$ set to $\rho_{m}$, if all the $T_{i j}$ 's are 1. From equation (2.19) it can be seen that this constraint requires, one of (i) $\lambda=1$, (ii) the horizontal distance between the boreholes to be infinite or at least very much larger than the vertical separation of the electrodes, or (iii) $z_{i}=z_{j}$. However, in sea ice $\lambda$ is less than one due to the horizontal resistivity being greater than the vertical 
resistivity. Furthermore a very large distance between the boreholes is logistically difficult, as signal strength is reduced as the separation of current electrodes is increased. Hence, the last alternative is the only feasible option and implies that all four electrodes must be at the same depth. It is undesirable to use the same electrode for both current injection and potential measurement. Therefore, to be able to make crossborehole resistivity measurements of $\rho_{m}$ with a four electrode array, it is necessary to have a minimum of 4 boreholes. The required electrode configuration is illustrated in Figure 2.4 below.

It is also possible to make measurements of the geometric mean resistivity using a three electrode array, with a remote electrode placed significantly far from the array $\left(\mid x_{i}-\right.$ $x_{j} \mid \rightarrow \infty$ and/or $\left.\left|y_{i}-y_{j}\right| \rightarrow \infty\right)$. In this situation the $T_{i j}$ term, as defined in equation (2.19), tends to 1 for $V_{i j}$ measurements involving the remote electrode. If the remaining three electrodes are placed at the same depth $\left(z_{i}=z_{j}\right)$ in their separate boreholes then again the $T_{i j}$ term tends to 1 . Thus with three electrodes positioned at the same depth in separate boreholes and a remote electrode (as shown in Figure 2.4), equation (2.20) still simplifies to equation (2.21) with $\rho=\rho_{m}$, and measurements of the geometric mean resistivity can be obtained.

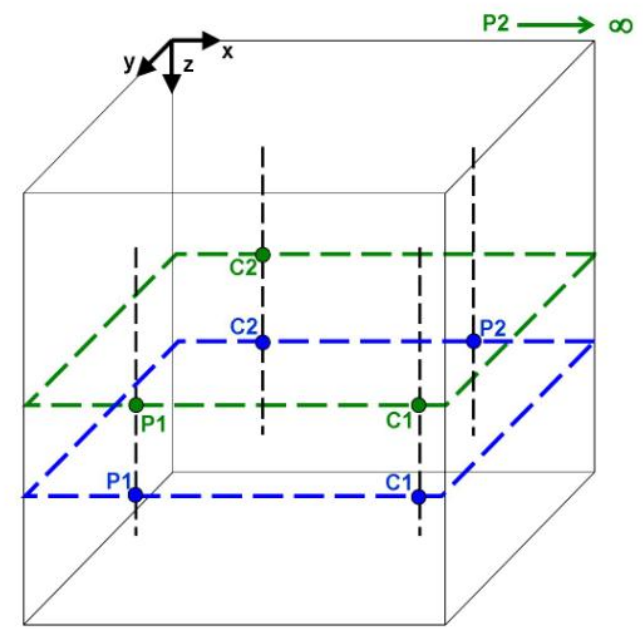

Figure 2.4: Electrode configuration for geometric mean resistivity measurements; with four electrodes at the same depth in separate boreholes, and three electrodes at the same depth in separate boreholes and a remote electrode.

Having two different arrays making measurements of the mean resistivity structure allows more data to be gathered giving better resolution. Furthermore the condition that 
$z_{i}=z_{j}$ can be lessened to $z_{i}-z_{j}$ is significantly smaller than $\sqrt{\left|x_{i}-x_{j}\right|^{2}+\left|y_{i}-y_{j}\right|^{2}}$ such that $T_{i j}$ approximates 1 . Table 2.1 below shows values of $z_{i}-z_{j}$ for which $T_{i j} \sim 1$ holds, indicating further measurements that can be made, over and above the $z_{i}=z_{j}$ measurements.

\begin{tabular}{|c|c|c|c|c|c|c|}
\hline \multirow[b]{2}{*}{$z_{i}-z_{j}(\mathbf{m})$} & \multicolumn{3}{|c|}{$\begin{array}{c}\text { Boreholes } i \text { and } j \text { located along } \\
\text { one side of square } \\
\left(x_{i}-x_{j}=0 \text { or } 1 \& y_{i}-y_{j}=1 \text { or } 0\right)\end{array}$} & \multicolumn{3}{|c|}{$\begin{array}{l}\text { Boreholes } i \text { and } j \text { located on } \\
\text { diagonal corners of square } \\
\quad\left(x_{i}-x_{j}=1 \& y_{i}-y_{j}=1\right)\end{array}$} \\
\hline & $T_{i j}(\lambda=0.1)$ & $T_{i j}(\lambda=0.2)$ & $T_{i j}(\lambda=0.3)$ & $T_{i j}(\lambda=\mathbf{0 . 1})$ & $T_{i j} \quad(\lambda=0.2)$ & $T_{i j}(\lambda=0.3)$ \\
\hline 0 & 1.00 & 1.00 & 1.00 & 1.00 & 1.00 & 1.00 \\
\hline 0.1 & 1.00 & 1.00 & 1.00 & 1.00 & 1.00 & 1.00 \\
\hline 0.2 & 1.02 & 1.02 & 1.02 & 1.01 & 1.01 & 1.01 \\
\hline 0.3 & 1.04 & 1.04 & 1.04 & 1.02 & 1.02 & 1.02 \\
\hline 0.4 & 1.08 & 1.07 & 1.07 & 1.04 & 1.04 & 1.04 \\
\hline 0.5 & 1.12 & 1.11 & 1.11 & 1.06 & 1.06 & 1.05 \\
\hline 0.6 & 1.16 & 1.16 & 1.15 & 1.09 & 1.08 & 1.08 \\
\hline 0.7 & 1.22 & 1.21 & 1.19 & 1.11 & 1.11 & 1.10 \\
\hline 0.8 & 1.28 & 1.26 & 1.25 & 1.15 & 1.14 & 1.13 \\
\hline 0.9 & 1.34 & 1.32 & 1.30 & 1.18 & 1.18 & 1.16 \\
\hline 1 & 1.41 & 1.39 & 1.35 & 1.22 & 1.21 & 1.20 \\
\hline 1.2 & 1.55 & 1.52 & 1.47 & 1.31 & 1.29 & 1.27 \\
\hline 1.3 & 1.63 & 1.59 & 1.53 & 1.35 & 1.34 & 1.31 \\
\hline 1.4 & 1.70 & 1.66 & 1.59 & 1.40 & 1.38 & 1.35 \\
\hline 1.5 & 1.78 & 1.73 & 1.64 & 1.45 & 1.43 & 1.39 \\
\hline 1.6 & 1.86 & 1.80 & 1.70 & 1.50 & 1.47 & 1.43 \\
\hline
\end{tabular}

Table 2.1: $T_{i j}$ values for different $z_{i}-z_{j}$ distances, at typical $\lambda$ values for first-year sea ice.

From the table it can be seen that for $\lambda \leq 0.3$ differences in $z_{i}$ and $z_{j}$ of $0.1 \mathrm{~m}, 0.2 \mathrm{~m}, 0.3 \mathrm{~m}$ and even $0.4 \mathrm{~m}$ still give a good approximation (to within $10 \%$ ) to $T_{i j}=1$ and thus allow measurements of the mean resistivity to be made in a medium with a small coefficient of anisotropy (eg. sea ice).

\subsubsection{Measurements of vertical resistivity $\left(\rho_{V}\right)$}

Equation (2.20) reduces to equation (2.21) with $\rho=\rho_{V}=\lambda \rho_{m}$ if all the $T_{i j}$ 's equal $\lambda$. This requires $\left|z_{i}-z_{j}\right|=\sqrt{\frac{-1}{\left(\lambda^{2}+1\right)}\left(\left|x_{i}-x_{j}\right|^{2}+\left|y_{i}-y_{j}\right|^{2}\right)}$ which clearly does not have a real solution. It may therefore be concluded that there are no possible cross-borehole 
measurements that can be interpreted in terms of the vertical component of the anisotropic bulk resistivity of sea ice.

The previous sections have shown that it is possible to make resistivity measurements, on an infinite anisotropic medium, which will provide approximations to the geometric mean and horizontal resistivities, using three to four electrodes in two to four boreholes. From these measurements the relationship between the geometric mean, horizontal and vertical resistivities will allow the vertical resistivity to be calculated (using equation (2.7) from section 2.1.2).

$$
\rho_{m}=\sqrt{\rho_{V} \rho_{H}} \Rightarrow \rho_{v}=\rho_{m}{ }^{2} / \rho_{H}
$$




\subsection{Cross-borehole resistivity measurements in a bounded anisotropic medium}

The expressions for the potential measured in an anisotropic medium (given by Bhattacharya and Patra, 1968 and Ingham et al., 2008, see equation (2.19) from section 2.2 above)

$$
V_{i j}=\frac{I \rho_{m}}{4 \pi}\left(\frac{1}{\sqrt{\left|x_{i}-x_{j}\right|^{2}+\left|y_{i}-y_{j}\right|^{2}+\lambda^{2}\left|z_{i}-z_{j}\right|^{2}}}\right)
$$

assume an infinite medium with no boundaries. Even in the simplest approximation, of a uniform bulk resistivity medium, sea ice is really a three layer system with two boundaries; the ice/air and ice/water interfaces. Although the above theory (section 2.2) suggests that measurement of the horizontal component and geometric mean resistivity are possible in an infinite anisotropic medium, this will not necessarily be the case in a bounded anisotropic medium. Synthetic data can be created for a simple three layer model of the sea ice in order to test whether the theoretical results, obtained from consideration of an infinite uniform anisotropic medium, still hold for this more realistic consideration of sea ice. That is, can the geometric mean and horizontal resistivity structure, used to generate the data, be accurately recovered from inversion of the

synthetic data using the code Res3dinv ${ }^{\mathrm{TM}}$ produced by Geotomo Software (see Chapter 4). However, in order to generate synthetic data it is necessary to see how the above results are modified by the inclusion of boundaries.

\subsubsection{Current flow through a boundary}

Telford et al. (1977) explain how the current flow through a boundary between two media is analogous to the optical case of a point light source in one medium separated from another by a semi-transparent mirror, with reflection coefficient $k$ and transmission coefficient $1-k$.

Consider the case shown below in Figure 2.5 with the current source at $C\left(x_{j}, y_{j}, z_{j}\right)$ in medium (1) and the potential measured at point $P_{l}\left(x_{i_{1}}, y_{i_{1}}, z_{i_{1}}\right)$ in medium (1) and point $P_{2}\left(x_{i_{2}}, y_{i_{2}},-z_{i_{2}}\right)$ in medium (2) 


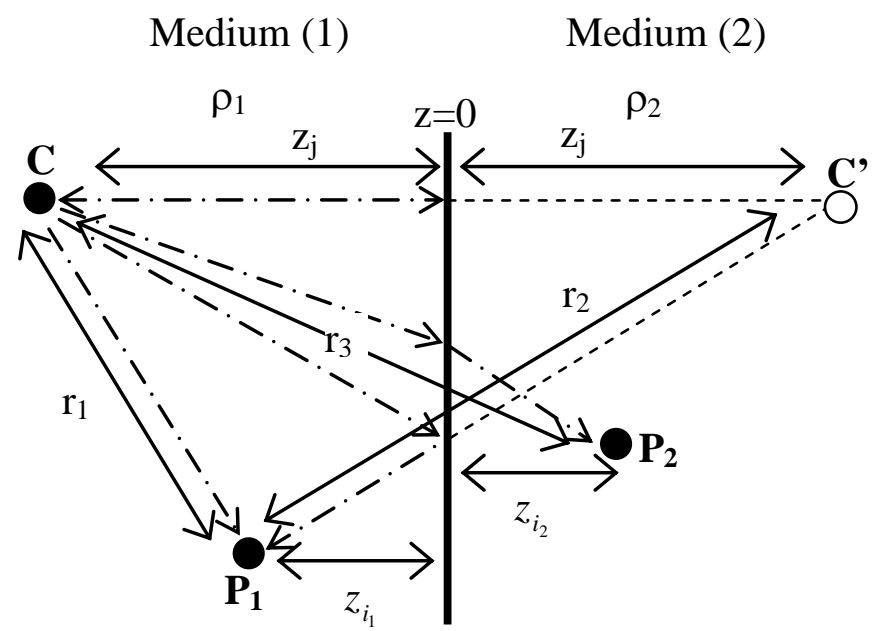

Figure 2.5: Point source in one medium separated from another medium by a semi-transparent mirror. Geometry for calculation of potential measured at a point in the first medium $\left(P_{l}\right)$ and at a point in the second medium $\left(P_{2}\right)$.

The intensity at a point in the first medium is a combination of the intensity due to the point source $(C)$ and the intensity due to its image in the second medium $\left(C^{\prime}\right.$ at $\left.\left(x_{j}, y_{j},-z_{j}\right)\right)$, diminished by the reflection coefficient $(k)$. The intensity at a point in the second medium is due only to the intensity of the point source $(C)$, diminished by the transmission coefficient $(1-k)$.

In this situation, and assuming isotropic media with resistivities $\rho_{1}$ and $\rho_{2}$, the potential at point $P_{1}$ is given by

$$
V_{1}=\frac{I \rho_{1}}{4 \pi}\left(\frac{1}{r_{1}}+\frac{k}{r_{2}}\right)
$$

where $r_{1}=\sqrt{\left|x_{i_{1}}-x_{j}\right|^{2}+\left|y_{i_{1}}-y_{j}\right|^{2}+\left|z_{i_{1}}-z_{j}\right|^{2}}$

$$
r_{2}=\sqrt{\left|x_{i_{1}}-x_{j}\right|^{2}+\left|y_{i_{1}}-y_{j}\right|^{2}+\mid z_{i_{1}}-\left(-z_{j}\right)^{2}}
$$

while the potential at $P_{2}$ is

$$
V_{2}=\frac{I \rho_{2}}{4 \pi}\left(\frac{1-k}{r_{3}}\right)
$$

where $r_{3}=\sqrt{\left|x_{i_{2}}-x_{j}\right|^{2}+\left|y_{i_{2}}-y_{j}\right|^{2}+\left|\left(-z_{i_{2}}\right)-z_{j}\right|^{2}}$

If $P_{1}$ and $P_{2}$ lie at a point on the boundary, then these potentials must be equal.

$$
\frac{I \rho_{1}}{4 \pi}\left(\frac{1}{r_{1}}+\frac{k}{r_{2}}\right)=\frac{I \rho_{2}}{4 \pi}\left(\frac{1-k}{r_{3}}\right)
$$


At an observation point on the boundary the distances $r_{1}, r_{2}$ and $r_{3}$ will be equal, thus the condition becomes

$$
\frac{I \rho_{1}}{4 \pi}\left(\frac{1+k}{r}\right)=\frac{I \rho_{2}}{4 \pi}\left(\frac{1-k}{r}\right) \Rightarrow \frac{\rho_{1}}{\rho_{2}}=\left(\frac{1-k}{1+k}\right)
$$

Rearranging gives the reflection coefficient $k$ of the boundary, which lies between \pm 1 depending on the relative resistivities of the two media.

$$
k=\left(\frac{\rho_{2}-\rho_{1}}{\rho_{2}+\rho_{1}}\right)
$$

In an anisotropic medium, such as sea ice, the expression for the potential changes from that obtained in an isotropic medium

$$
V_{i j}=\frac{I \rho}{4 \pi r_{i j}}=\frac{I \rho}{4 \pi}\left(\frac{1}{\sqrt{\left|x_{i}-x_{j}\right|^{2}+\left|y_{i}-y_{j}\right|^{2}+\left|z_{i}-z_{j}\right|^{2}}}\right)
$$

to

$$
V_{i j}=\frac{I \rho_{m}}{4 \pi}\left(\frac{1}{\sqrt{\left|x_{i}-x_{j}\right|^{2}+\left|y_{i}-y_{j}\right|^{2}+\lambda^{2}\left|z_{i}-z_{j}\right|^{2}}}\right)
$$

meaning equations (2.23) and (2.24) become

$$
V_{1}=\frac{I \rho_{m 1}}{4 \pi}\left(\begin{array}{l}
\frac{1}{\sqrt{\left|x_{i_{1}}-x_{j}\right|^{2}+\left|y_{i_{1}}-y_{j}\right|^{2}+\lambda^{2}\left|z_{i_{1}}-z_{j}\right|^{2}}} \\
+\frac{k}{\sqrt{\left|x_{i_{1}}-x_{j}\right|^{2}+\left|y_{i_{1}}-y_{j}\right|^{2}+\lambda^{2}\left|z_{i_{1}}+z_{j}\right|^{2}}}
\end{array}\right)
$$

and

$$
V_{2}=\frac{I \rho_{m 2}}{4 \pi}\left(\frac{1-k}{\sqrt{\left|x_{i_{2}}-x_{j}\right|^{2}+\left|y_{i_{2}}-y_{j}\right|^{2}+\lambda^{2}\left|\left(-z_{i_{2}}\right)-z_{j}\right|^{2}}}\right)
$$

\subsubsection{Three layer model}

The analogy above can be extended to a three layer model with two boundaries, as in a simple model for sea ice with three media - air, ice and water and two interfaces ice/air and ice/water. 
The method of calculating the potential measured in a medium consisting of three layers of differing isotropic resistivity structures can be found in Keller and Frischknecht (1966) and is summarised in the following.

A three layer system is shown below in Figure 2.6, with; a layer of thickness $t$ and resistivity $\rho_{l}$ bounded above by a semi-infinite space with resistivity $\rho_{0}$ and below by a semi-infinite space with a resistivity of $\rho_{2}$. A current source $(C)$ is located a distance, $h$, beneath the upper boundary, and an observation point $(P)$ is located the same distance below the upper boundary, but at a horizontal distance, $a$, from the current source.

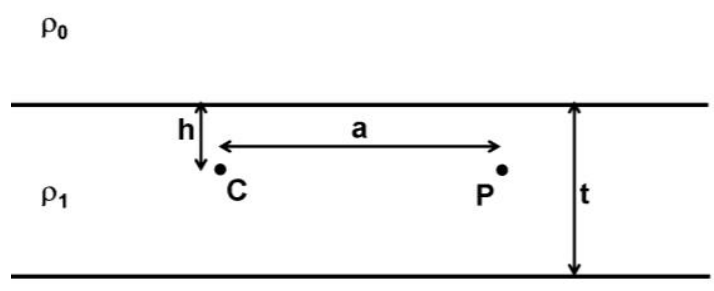

$\rho_{2}$

Figure 2.6: Structure consisting of three isotropic resistivity layers, with resistivities $\rho_{0}, \rho_{l}$ and $\rho_{2}$. Potential, due to current injected at $C$, is measured at point $P$.

In this situation current from the source $C$ reaches point $P$ either via a direct path or a path made up of infinite reflections from both the upper and lower boundaries (see Figure 2.7 below). The image of $C$ due to reflection in the bottom boundary is labelled as $C_{1}{ }^{(2)}$, where the superscript refers to the medium in which the image source appears to be and the subscript indicates the number of reflections the ray path has been through. The original image is then reflected in the top boundary and labelled as $C_{2}{ }^{(0)}$, this image is then reflected in the bottom boundary and so on. A similar set of images forms from $C$ being reflected in the top boundary. The intensity of the potential measured at $P$ due to these images is diminished by a value determined by the number of reflections and the reflection coefficients of the two boundaries. 


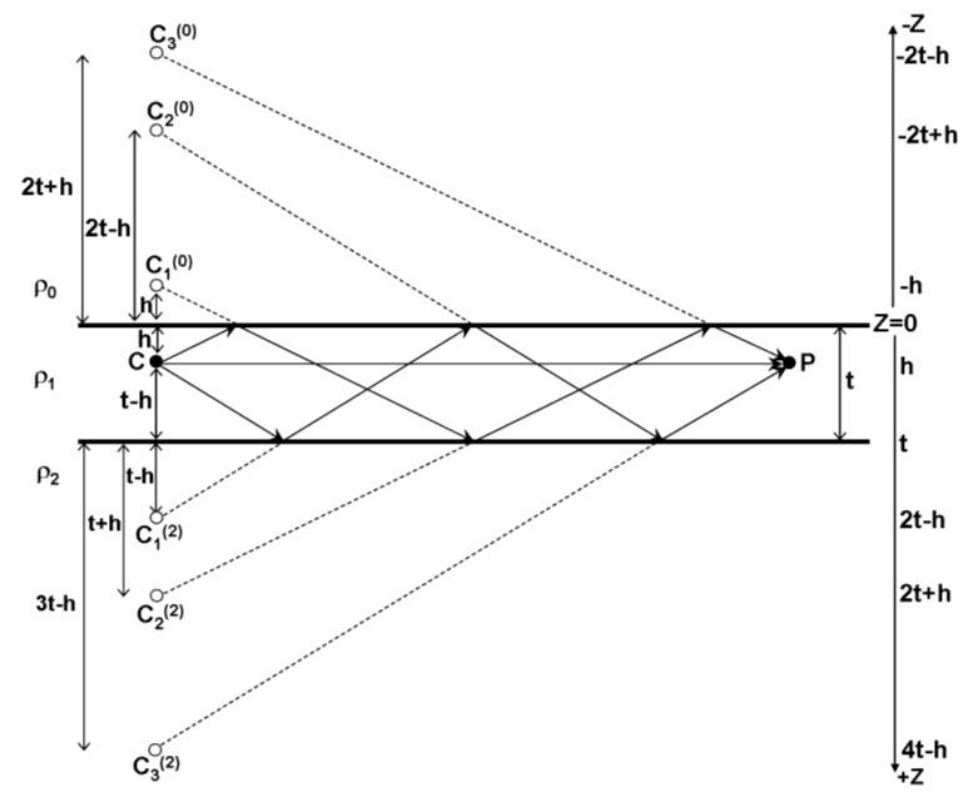

Figure 2.7: Current paths between current source $C$ and measurement point $P$, and apparent locations of the image source.

As indicated in Figure 2.7 above the simplest path the current follows is the direct route between $C$ and $P$. In this case the potential measured at $P$ is due to the primary source at $C$ and is given by

$$
V_{0}=\frac{\rho_{1} I}{4 \pi a}
$$

Now consider the situations where the ray path is reflected an even number of times, with the first reflection being from the bottom boundary. Examples of this case with paths containing two and four reflections are shown in Figure 2.8 below.

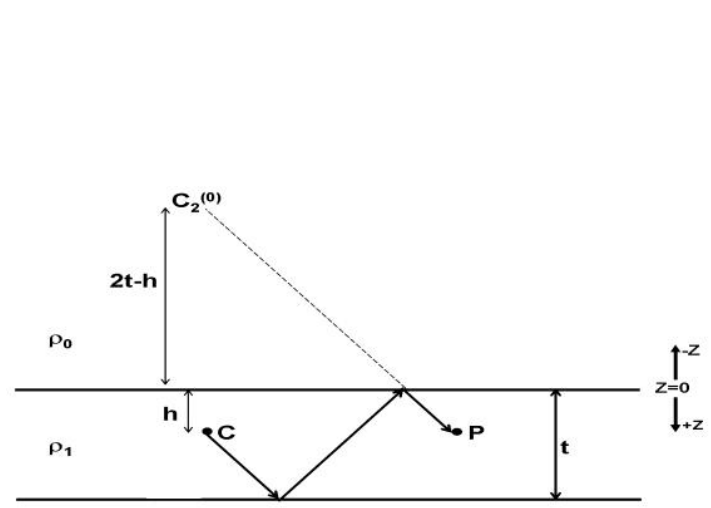

$\rho_{2}$

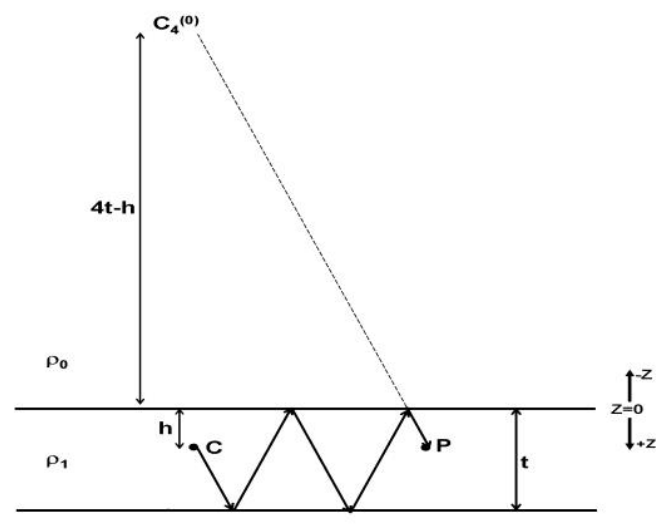

$\rho_{2}$

(a)

(b)

Figure 2.8: Examples of ray paths travelling from $C$ to $P$ via an even number of reflections, with the first reflection being from the bottom boundary (a) displays the situation of two reflections while path (b) follows four reflections. 
For a path with one reflection from each boundary, Figure 2.8(a), the image source appears to be in medium 0 and has an intensity which is diminished by $k_{1,2} k_{1,0}$. The variable $k_{\mathrm{ij}}$ is the reflection coefficient of the boundary between media $i$ and $j$, for ray paths travelling from medium $i$ to $j$ and is given by $k_{i j}=\left(\frac{\rho_{j}-\rho_{i}}{\rho_{j}+\rho_{i}}\right)$ as indicated in expression (2.25) above. The contribution to the total potential at point $P$ from this image is

$$
V_{2}^{(0)}=\frac{\rho_{1} I k_{1,2} k_{1,0}}{4 \pi \sqrt{a^{2}+(2 t)^{2}}}
$$

where the superscript of $V_{2}^{(0)}$ refers to the medium in which the image source appears to be and the subscript indicates the number of reflections the ray path has been through.

For a path with two reflections from both the upper and lower boundaries, Figure 2.8(b), the image source appears to be in medium 0 and has an intensity which is diminished by $k_{1,2}^{2} k_{1,0}^{2}$. This image appears to be a distance $2 t$ further above the upper boundary than the preceding image. The contribution to the potential at $P$ due to this image is

$$
V_{4}^{(0)}=\frac{\rho_{1} I k_{1,2}{ }^{2} k_{1,0}{ }^{2}}{4 \pi \sqrt{a^{2}+(4 t)^{2}}}
$$

For each additional pair of reflections, one from each of the boundaries, the corresponding image strength will be further reduced by the factor $k_{1,2} k_{1,0}$ and will appear to be a distance $2 t$ further above the upper boundary, than the previous image. Hence it is possible to write an infinite series representing the potential due to images corresponding to ray paths reflected an even number of times, the first reflection being from the lower boundary.

$$
V_{I}=\sum_{n=1}^{\infty} \frac{\rho_{1} I k_{1,2}{ }^{n} k_{1,0}{ }^{n}}{4 \pi \sqrt{a^{2}+(2 n t)^{2}}}
$$

Similarly, a series of images for ray paths with an odd number of reflections, the first being from the lower boundary may be constructed (e.g. Figure 2.9) 

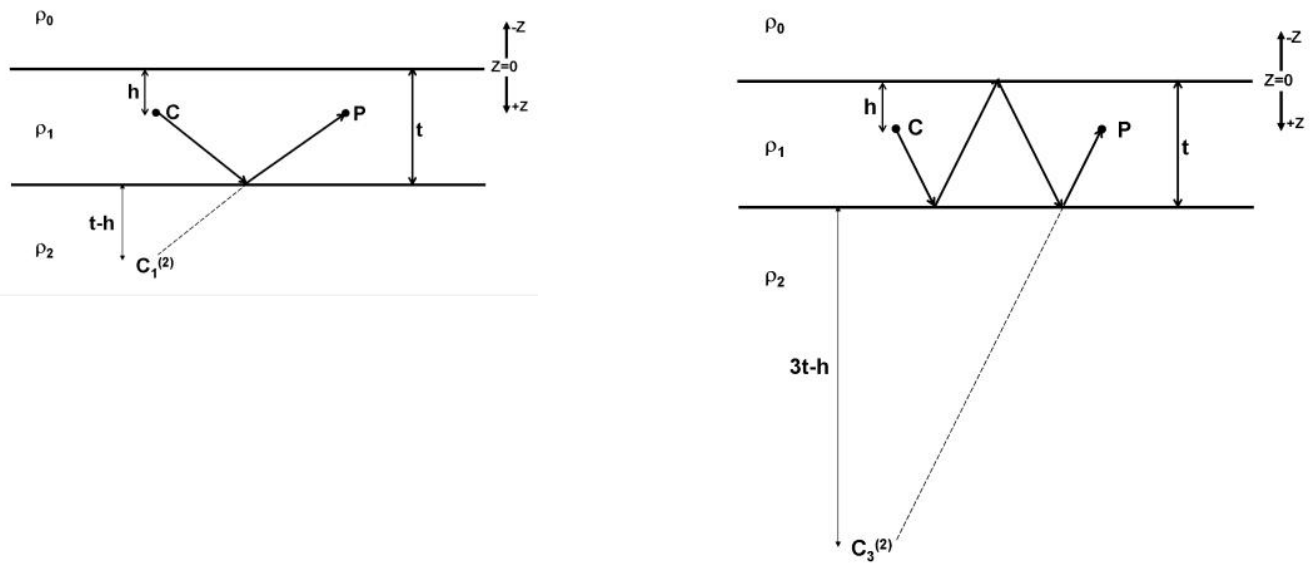

Figure 2.9: Examples of ray paths travelling from $C$ to $P$ via an odd number of reflections, with the first reflection being from the bottom boundary.

The potential due to an infinite series of such images is

$$
V_{I I}=\sum_{n=1}^{\infty} \frac{\rho_{1} I k_{1,2}{ }^{n} k_{1,0}{ }^{(n-1)}}{4 \pi \sqrt{a^{2}+(-2 n t+2 h)^{2}}}
$$

There may also be ray paths with an even (Figure 2.10(a)) or odd (Figure 2.10(b)) number of reflections with the first being from the upper rather than the lower boundary
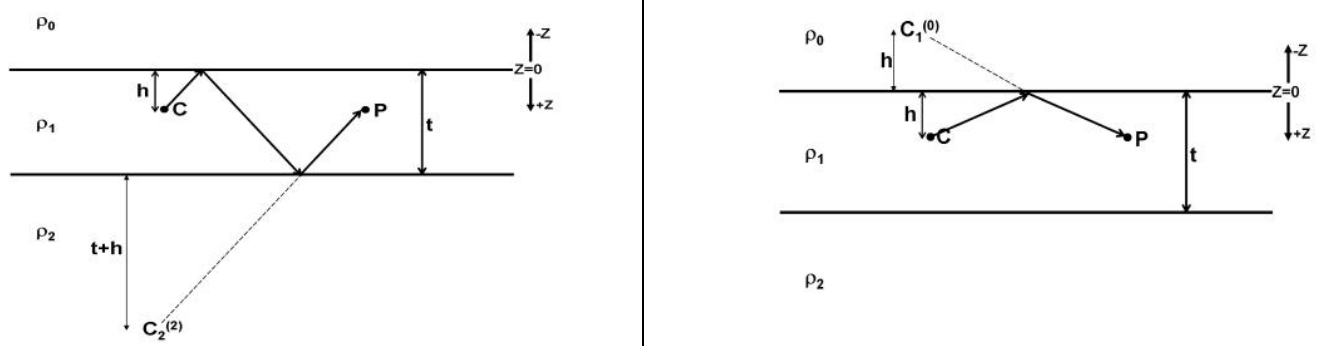

$\rho_{2}$
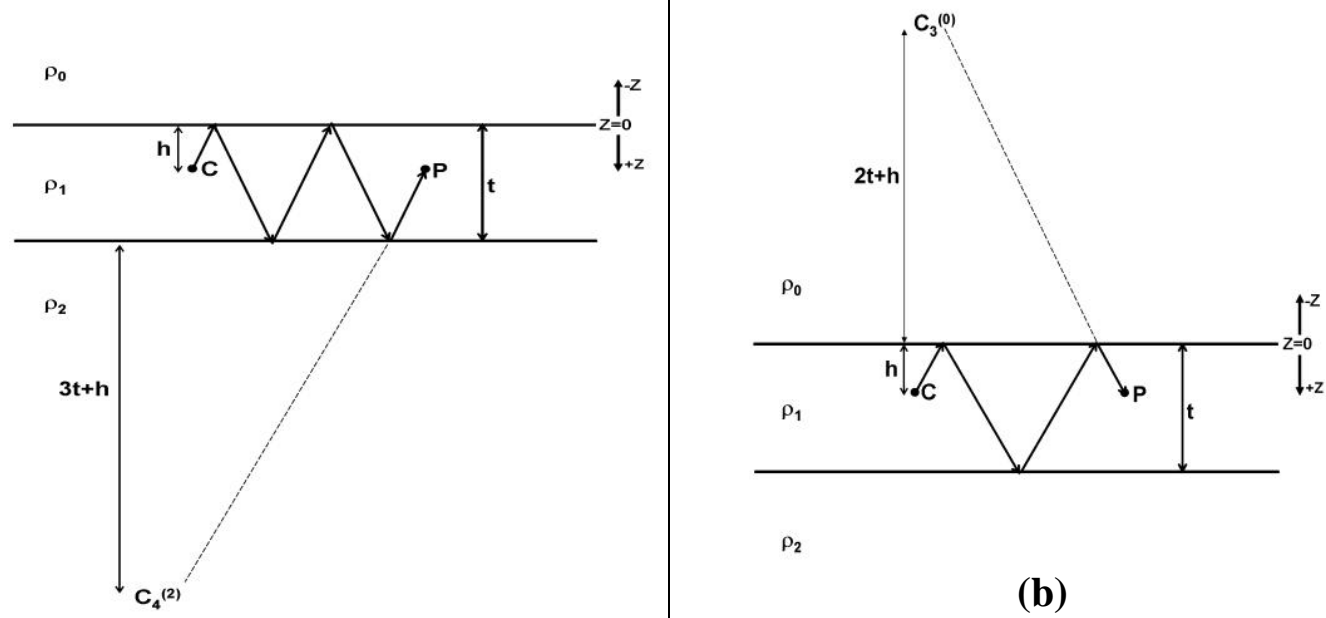

$\rho_{2}$

(b)

Figure 2.10: Examples of ray paths travelling from $C$ to $P$, with the first reflection always being from the top boundary. (a) an even number of reflections and (b) an odd number of reflections. 
The potential measured at $P$ due to an infinite series of images produced by ray paths reflected an even number of times, the first reflection being from the upper boundary as in Figure 2.10(a), is given by

$$
V_{I I I}=\sum_{n=1}^{\infty} \frac{\rho_{1} I k_{1,0}{ }^{n} k_{1,2}{ }^{n}}{4 \pi \sqrt{a^{2}+(-2 n t)^{2}}}
$$

while the contribution to the total potential at $P$ due to ray paths reflected an odd number of times, the first reflection being from the upper boundary as in Figure 2.10(b), is given by

$$
V_{I V}=\sum_{n=1}^{\infty} \frac{\rho_{1} I k_{1,0}{ }^{n} k_{1,2}{ }^{(n-1)}}{4 \pi \sqrt{a^{2}+(2(n-1) t+2 h)^{2}}}
$$

The total potential measured at point $P$ is found by adding all the terms included in equations (2.28) - (2.32).

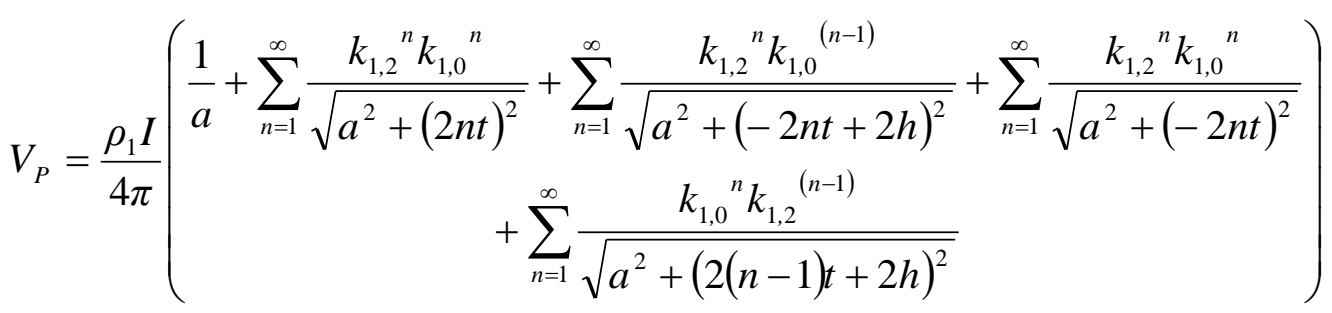

(2.33)

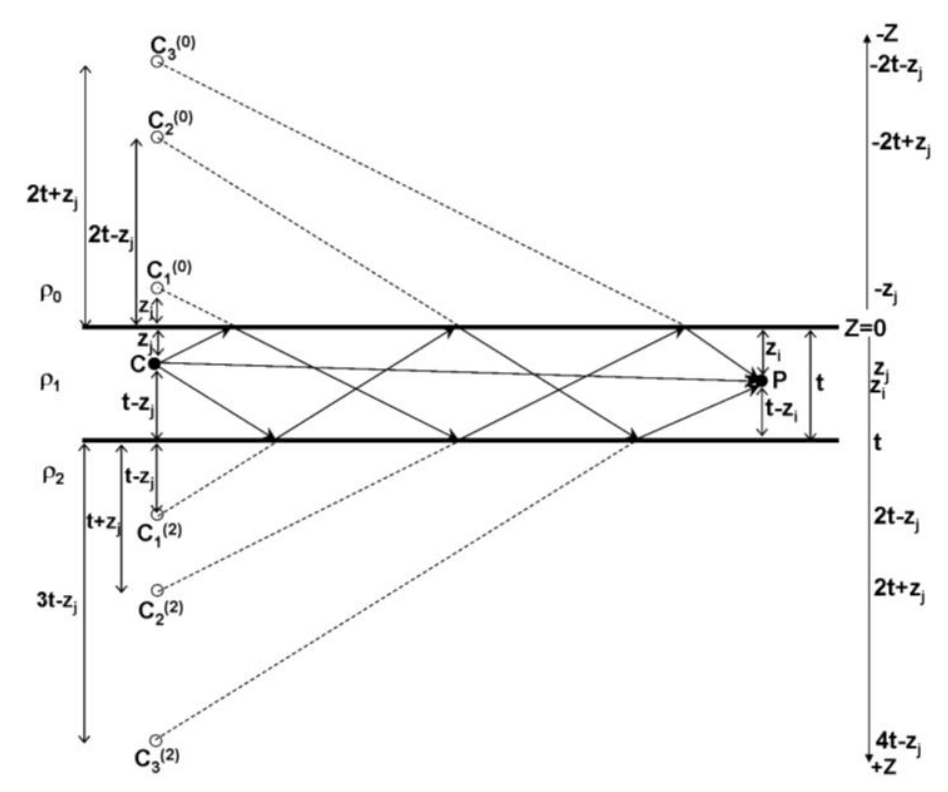

Figure 2.11: Current paths travelling from current electrode $C$, at a depth of $z_{j}$ below the upper boundary to potential electrode $P$, a horizontal distance $a$ away at a depth of $z_{i}$, in a three layer medium. 
If the current $(C)$ and potential $(P)$ electrodes, separated by a horizontal distance $a=\sqrt{\left|x_{i}-x_{j}\right|^{2}+\left|y_{i}-y_{j}\right|^{2}}$, are not at the same depth $h$ below the upper boundary but rather at depths of $z_{j}$ and $z_{i}$ respectively, as shown in Figure 2.11 above, then expressions (2.28) - (2.32) become

$$
\begin{aligned}
& V_{0}=\frac{\rho_{1} I}{4 \pi \sqrt{\left|x_{i}-x_{j}\right|^{2}+\left|y_{i}-y_{j}\right|^{2}+\left|z_{i}-z_{j}\right|^{2}}} \\
& V_{I}=\sum_{n=1}^{\infty} \frac{\rho_{1} I k_{1,2}{ }^{n} k_{1,0}{ }^{n}}{4 \pi \sqrt{\left|x_{i}-x_{j}\right|^{2}+\left|y_{i}-y_{j}\right|^{2}+\left|2 n t+z_{i}-z_{j}\right|^{2}}} \\
& V_{I I}=\sum_{n=1}^{\infty} \frac{\rho_{1} I k_{1,2}{ }^{n} k_{1,0}{ }^{(n-1)}}{4 \pi \sqrt{\left|x_{i}-x_{j}\right|^{2}+\left|y_{i}-y_{j}\right|^{2}+\left|-2 n t+z_{i}+z_{j}\right|^{2}}} \\
& V_{I I I}=\sum_{n=1}^{\infty} \frac{\rho_{1} I k_{1,0}{ }^{n} k_{1,2}{ }^{n}}{4 \pi \sqrt{\left|x_{i}-x_{j}\right|^{2}+\left|y_{i}-y_{j}\right|^{2}+\left|-2 n t+z_{i}-z_{j}\right|^{2}}} \\
& V_{I V}=\sum_{n=1}^{\infty} \frac{\rho_{1} I k_{1,0}{ }^{n} k_{1,2}{ }^{(n-1)}}{4 \pi \sqrt{\left|x_{i}-x_{j}\right|^{2}+\left|y_{i}-y_{j}\right|^{2}+\left|2(n-1) t+z_{i}+z_{j}\right|^{2}}}
\end{aligned}
$$

Furthermore if medium one has an anisotropic rather than isotropic resistivity structure then the expression for the potential changes from that obtained in an isotropic medium

$$
V_{i j}=\frac{I \rho}{4 \pi r_{i j}}=\frac{I \rho}{4 \pi}\left(\frac{1}{\sqrt{\left|x_{i}-x_{j}\right|^{2}+\left|y_{i}-y_{j}\right|^{2}+\left|z_{i}-z_{j}\right|^{2}}}\right)
$$

to

$$
V_{i j}=\frac{I \rho_{m}}{4 \pi}\left(\frac{1}{\sqrt{\left|x_{i}-x_{j}\right|^{2}+\left|y_{i}-y_{j}\right|^{2}+\lambda^{2}\left|z_{i}-z_{j}\right|^{2}}}\right)
$$

In this case expressions (2.28) - (2.32) become

$$
\begin{aligned}
& V_{0}=\frac{\rho_{m 1} I}{4 \pi \sqrt{\left|x_{i}-x_{j}\right|^{2}+\left|y_{i}-y_{j}\right|^{2}+\lambda^{2}\left|z_{i}-z_{j}\right|^{2}}} \\
& V_{I}=\sum_{n=1}^{\infty} \frac{\rho_{m 1} I k_{1,2}{ }^{n} k_{1,0}{ }^{n}}{4 \pi \sqrt{\left|x_{i}-x_{j}\right|^{2}+\left|y_{i}-y_{j}\right|^{2}+\lambda^{2}\left|2 n t+z_{i}-z_{j}\right|^{2}}} \\
& V_{I I}=\sum_{n=1}^{\infty} \frac{\rho_{m 1} I k_{1,2}{ }^{n} k_{1,0}{ }^{(n-1)}}{4 \pi \sqrt{\left|x_{i}-x_{j}\right|^{2}+\left|y_{i}-y_{j}\right|^{2}+\lambda^{2}\left|-2 n t+z_{i}+z_{j}\right|^{2}}}
\end{aligned}
$$




$$
\begin{aligned}
V_{I I I} & =\sum_{n=1}^{\infty} \frac{\rho_{m 1} I k_{1,0}{ }^{n} k_{1,2}{ }^{n}}{4 \pi \sqrt{\left|x_{i}-x_{j}\right|^{2}+\left|y_{i}-y_{j}\right|^{2}+\lambda^{2}\left|-2 n t+z_{i}-z_{j}\right|^{2}}} \\
V_{I V} & =\sum_{n=1}^{\infty} \frac{\rho_{m 1} I k_{1,0}{ }^{n} k_{1,2}{ }^{(n-1)}}{4 \pi \sqrt{\left|x_{i}-x_{j}\right|^{2}+\left|y_{i}-y_{j}\right|^{2}+\lambda^{2}\left|2(n-1) t+z_{i}+z_{j}\right|^{2}}}
\end{aligned}
$$

These expressions can be simplified if the upper half-space is air with an infinite resistivity. In this situation the reflection coefficient $k_{1,0}$, using equation (2.25), is given by

$$
k_{1,0}=\frac{\rho_{0}-\rho_{1}}{\rho_{0}+\rho_{1}} \sim \frac{\rho_{0}}{\rho_{0}}=1
$$

Hence

$$
\begin{aligned}
& V_{0}=\frac{\rho_{m 1} I}{4 \pi \sqrt{\left|x_{i}-x_{j}\right|^{2}+\left|y_{i}-y_{j}\right|^{2}+\lambda^{2}\left|z_{i}-z_{j}\right|^{2}}} \\
& V_{I}=\sum_{n=1}^{\infty} \frac{\rho_{m 1} I k_{1,2}{ }^{n}}{4 \pi \sqrt{\left|x_{i}-x_{j}\right|^{2}+\left|y_{i}-y_{j}\right|^{2}+\lambda^{2}\left|2 n t+z_{i}-z_{j}\right|^{2}}} \\
& V_{I I}=\sum_{n=1}^{\infty} \frac{\rho_{m 1} I k_{1,2}{ }^{n}}{4 \pi \sqrt{\left|x_{i}-x_{j}\right|^{2}+\left|y_{i}-y_{j}\right|^{2}+\lambda^{2}\left|-2 n t+z_{i}+z_{j}\right|^{2}}} \\
& V_{I I I}=\sum_{n=1}^{\infty} \frac{\rho_{m 1} I k_{1,2}{ }^{n}}{4 \pi \sqrt{\left|x_{i}-x_{j}\right|^{2}+\left|y_{i}-y_{j}\right|^{2}+\lambda^{2}\left|-2 n t+z_{i}-z_{j}\right|^{2}}} \\
& V_{I V}=\sum_{n=1}^{\infty} \frac{\rho_{m 1} I k_{1,2}{ }^{(n-1)}}{4 \pi \sqrt{\left|x_{i}-x_{j}\right|^{2}+\left|y_{i}-y_{j}\right|^{2}+\lambda^{2}\left|2(n-1) t+z_{i}+z_{j}\right|^{2}}}
\end{aligned}
$$

Thus it can be shown that for a current, potential electrode pair positioned at $\left(x_{j}, y_{j}, z_{j}\right)$ and $\left(x_{i}, y_{i}, z_{i}\right)$ respectively within a layer of anisotropic sea ice of thickness $t$, bounded above by a half-space of highly resistive air and below by a half-space of highly conductive seawater, the potential measured at point $i$ due to current from point $j$ can be found by using the above modified versions of expressions (2.28) - (2.32), giving the following version of expression (2.33) 


$$
V_{i j}=\frac{\rho_{m} I}{4 \pi}\left(\begin{array}{c}
\frac{1}{\sqrt{\left|x_{i}-x_{j}\right|^{2}+\left|y_{i}-y_{j}\right|^{2}+\lambda^{2}\left|z_{i}-z_{j}\right|^{2}}} \\
+\sum_{n=1}^{\infty} \frac{k_{1,2}{ }^{n}}{\sqrt{\left|x_{i}-x_{j}\right|^{2}+\left|y_{i}-y_{j}\right|^{2}+\lambda^{2}\left|2 n t+z_{i}-z_{j}\right|^{2}}} \\
+\sum_{n=1}^{\infty} \frac{k_{1,2}{ }^{n}}{\sqrt{\left|x_{i}-x_{j}\right|^{2}+\left|y_{i}-y_{j}\right|^{2}+\lambda^{2}\left|-2 n t+z_{i}+z_{j}\right|^{2}}} \\
+\sum_{n=1}^{\infty} \frac{k_{1,2}{ }^{n}}{\sqrt{\left|x_{i}-x_{j}\right|^{2}+\left|y_{i}-y_{j}\right|^{2}+\lambda^{2}\left|-2 n t+z_{i}-z_{j}\right|^{2}}} \\
\sqrt{\left|x_{i}-x_{j}\right|^{2}+\left|y_{i}-y_{j}\right|^{2}+\lambda^{2}\left|2(n-1) t+z_{i}+z_{j}\right|^{2}}
\end{array}\right)
$$

The expression for the total potential between electrodes $i$ and $j$ (as given in equation (2.34)) can become further complicated by the fact that the electrodes can either be in the ice and/or the water. Thus the following configurations need to be considered when calculating the potential that will be measured in a field arrangement.

i. Both the current and potential electrode are in the ice (see Figure 2.11 above), in which case the total potential measured between electrodes is given by equation (2.34) above.

ii. Both the current and potential electrodes are in the water (see Figure 2.12 below), in which case the potential measured depends upon: (a) a direct ray path; (b) a ray path reflected off the water/ice interface whose intensity will be diminished by the reflection coefficient $k_{2,1}$; and (c) a series of ray paths transmitted through the water/ice boundary and reflected a odd number of times, with the first reflection off the air/ice boundary, before transmitting back through the water/ice boundary. In this case transmission through the water/ice boundary will decrease the intensity of the ray by the transmission coefficients $\left(1-k_{2,1}\right)$ and $\left(1-k_{1,2}\right)$, while reflection will diminish the intensity by $k_{1,0}{ }^{\mathrm{n}} k_{1,2}{ }^{(\mathrm{n}-1)}$ (which equals $k_{1,2}{ }^{(\mathrm{n}-1)}$ as $k_{1,0}{ }^{\mathrm{n}}=1$ ). As the potential electrode is in the seawater, which can be considered as an isotropic medium, the potential measured will be given by the expression below, where the resistivity $\rho$ will be the resistivity of the seawater. 


$$
V_{i j}=\frac{I \rho}{4 \pi}\left[\begin{array}{l}
\frac{1}{\sqrt{\left|x_{i}-x_{j}\right|^{2}+\left|y_{i}-y_{j}\right|^{2}+\left|z_{i}-z_{j}\right|^{2}}}+\frac{k_{2,1}}{\sqrt{\left|x_{i}-x_{j}\right|^{2}+\left|y_{i}-y_{j}\right|^{2}+\left|-2 t+z_{i}+z_{j}\right|^{2}}} \\
+\sum_{n=1}^{\infty} \frac{\left(1-k_{2,1}\right)\left(1-k_{1,2}\right) k_{1,2}(n-1)}{\sqrt{\left|x_{i}-x_{j}\right|^{2}+\left|y_{i}-y_{j}\right|^{2}+\left|2(n-1) t+z_{i}+z_{j}\right|^{2}}}
\end{array}\right]
$$

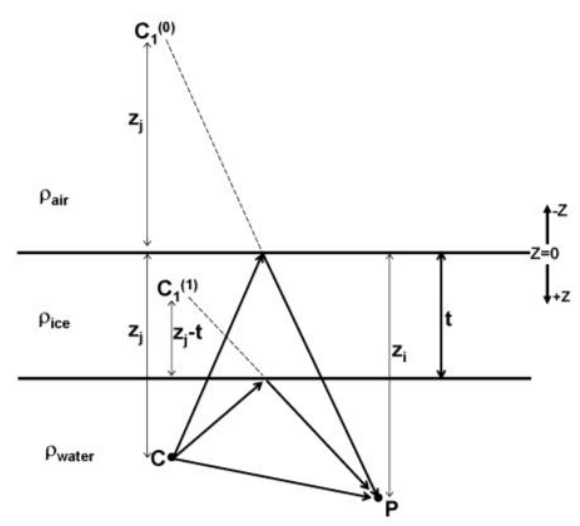

Figure 2.12: Current paths travelling from current electrode $C$ to potential electrode $P$, both in the seawater.

iii. The current electrode in the ice and the potential electrode in the water (see Figure 2.13 below), in which case the equation for the potential will involve: (a) a direct ray path, whose intensity will be diminished by the transmission coefficient $\left(1-k_{1,2}\right)$; (b) an infinite series of images produced by ray paths reflected an even number of times, the first reflection being from the lower boundary, then transmitted through the water/ice boundary. In this case the intensity will be diminished by $k_{1,0}{ }^{\mathrm{n}} k_{1,2}{ }^{\mathrm{n}}$ (which equals $k_{1,2}{ }^{\mathrm{n}}$ as $k_{1,0}{ }^{\mathrm{n}}=1$ ) from the reflections from the upper and lower boundaries and by the transmission coefficient $\left(1-k_{1,2}\right)$. Finally (c) an infinite series of images produced by ray paths reflected an odd number of times, the first reflection being from the upper boundary, then transmitted through the water/ice boundary. In this last situation the magnitude of the potential will be reduced by $k_{1,0}{ }^{\mathrm{n}} k_{1,2}{ }^{(\mathrm{n}-1)}$ (which equals $k_{1,2}^{(n-1)}$ as $k_{1,0}{ }^{\mathrm{n}}=1$ ) from the reflections with the boundaries and by the transmission coefficient $\left(1-k_{1,2}\right)$. As the seawater can be considered as an isotropic medium, and the potential electrode is in this medium, the measured potential will be given as below, where the resistivity $\rho$ will be the resistivity of the seawater. 


$$
V_{i j}=\frac{I \rho}{4 \pi}\left[\begin{array}{l}
\frac{\left(1-k_{1,2}\right)}{\sqrt{\left|x_{i}-x_{j}\right|^{2}+\left|y_{i}-y_{j}\right|^{2}+\left|z_{i}-z_{j}\right|^{2}}} \\
+\sum_{n=1}^{\infty} \frac{\left(1-k_{1,2}\right) k_{1,2}{ }^{n}}{\sqrt{\left|x_{i}-x_{j}\right|^{2}+\left|y_{i}-y_{j}\right|^{2}+\left|2 n t+z_{i}-z_{j}\right|^{2}}} \\
+\sum_{n=1}^{\infty} \frac{\left(1-k_{1,2}\right) k_{1,2}{ }^{(n-1)}}{\sqrt{\left|x_{i}-x_{j}\right|^{2}+\left|y_{i}-y_{j}\right|^{2}+\left|2(n-1) t+z_{i}+z_{j}\right|^{2}}}
\end{array}\right]
$$

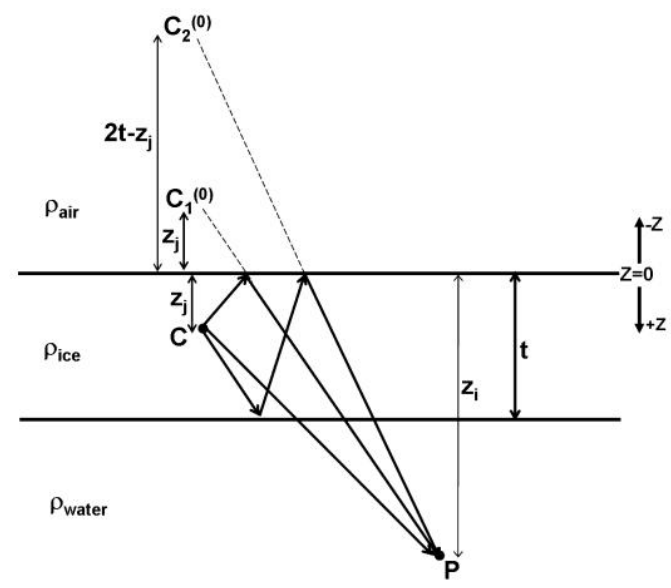

Figure 2.13: Current paths travelling from current electrode $C$, in the sea ice, to potential electrode $P$, in the seawater.

iv. The current electrode in the water and the potential electrode in the ice (see Figure 2.14 below), in which case the equation of the potential will involve: (a) a direct ray path, with an intensity diminished by the transmission coefficient $\left(1-k_{2,1}\right)$; (b) an infinite series of images produced by ray paths transmitted through the water/ice boundary then reflected an even number of times, the first reflection being from the upper boundary. In this case the intensity will be diminished by the transmission coefficient $\left(1-k_{2,1}\right)$ and by $k_{1,0}{ }^{\mathrm{n}} k_{1,2}{ }^{\mathrm{n}}$ (which equals $k_{1,2}{ }^{\mathrm{n}}$ as $k_{1,0}{ }^{\mathrm{n}}=1$ ) from the reflections from the upper and lower boundaries. Finally (c) an infinite series of images produced by ray paths transmitted through the water/ice boundary then reflected an odd number of times, the first reflection being from the upper boundary. In this last situation the magnitude of the potential will be reduced by the transmission coefficient $\left(1-k_{2,1}\right)$ and by $k_{1,0}{ }^{\mathrm{n}} k_{1,2}{ }^{(\mathrm{n}-1)}$ (which equals $k_{1,2}{ }^{(\mathrm{n}-1)}$ as 
$\left.k_{1,0}{ }^{\mathrm{n}}=1\right)$ from the reflections with the boundaries. As with the first electrode arrangement (i) the potential electrode is in the anisotropic sea ice and the measured potential is given by the expression below, where the resistivity $\rho_{m}$ is the geometric mean resistivity of the sea ice.

$$
V_{i j}=\frac{I \rho_{m}}{4 \pi}\left[\begin{array}{l}
\frac{\left(1-k_{2,1}\right)}{\sqrt{\left|x_{i}-x_{j}\right|^{2}+\left|y_{i}-y_{j}\right|^{2}+\lambda^{2}\left|z_{i}-z_{j}\right|^{2}}} \\
+\sum_{n=1}^{\infty} \frac{\left(1-k_{2,1}\right) k_{1,2}{ }^{n}}{\sqrt{\left|x_{i}-x_{j}\right|^{2}+\left|y_{i}-y_{j}\right|^{2}+\lambda^{2}\left|-2 n t+z_{i}-z_{j}\right|^{2}}} \\
+\sum_{n=1}^{\infty} \frac{\left(1-k_{2,1}\right) k_{1,2}{ }^{(n-1)}}{\sqrt{\left|x_{i}-x_{j}\right|^{2}+\left|y_{i}-y_{j}\right|^{2}+\lambda^{2}\left|2(n-1) t+z_{i}+z_{j}\right|^{2}}}
\end{array}\right]
$$

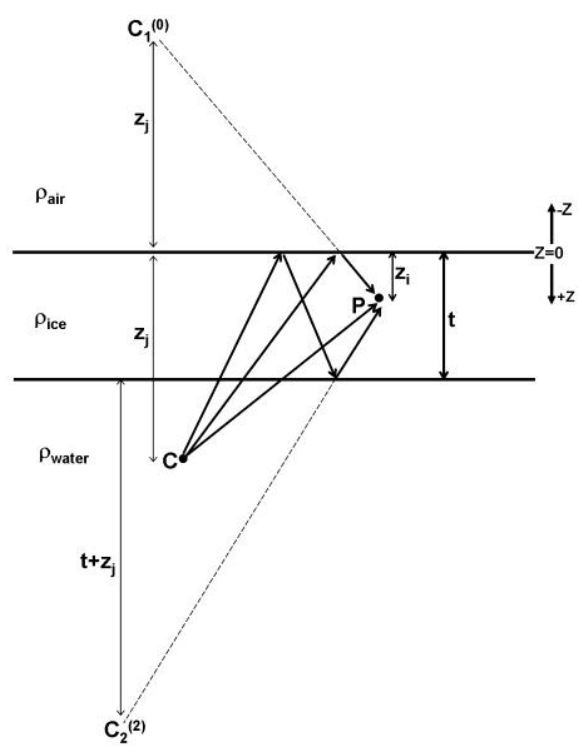

Figure 2.14: Current paths travelling from current electrode $C$, in the seawater, to potential electrode $P$, in the sea ice.

\subsubsection{Horizontal resistivity measurements $\left(\rho_{H}\right)$}

In order to make measurements of the horizontal component of the resistivity structure it is required to use two boreholes, each containing one current and one potential electrode. However, up to this point, an infinite medium was assumed instead of the more realistic three layer model of the sea ice. By considering the situation where both the electrodes are in the ice and applying the required configuration constraints to the relevant expression for the potential in a three layer model (equation (2.34)), it will be possible to determine whether the resistivity measurements made on sea ice will return 
$\rho_{H}$ as expected (for an infinite medium) or if the resistivity is dependent upon other factors. The current and potential electrodes are either (i) in the same borehole, in which case $\left|x_{i}-x_{j}\right|^{2}+\left|y_{i}-y_{j}\right|^{2}=0$ : (ii) in separate boreholes along a side of the $1 \mathrm{~m}$ by $1 \mathrm{~m}$ square, in which case $\left|x_{i}-x_{j}\right|^{2}+\left|y_{i}-y_{j}\right|^{2}=1$; or (iii) in separate boreholes along a diagonal of the $1 \mathrm{~m}$ by $1 \mathrm{~m}$ square, in which case $\left|x_{i}-x_{j}\right|^{2}+\left|y_{i}-y_{j}\right|^{2}=2$. For these three different cases equation (2.34) becomes:

(i) $\left.V_{i j}=\frac{I \rho_{H}}{4 \pi} \mid \begin{array}{l}+\sum_{n=1}^{\infty} \frac{k_{1,2}{ }^{n}}{\left(-2 n t+z_{i}+z_{j}\right)} \\ +\sum_{n=1}^{\infty} \frac{k_{1,2}{ }^{n}}{\left(-2 n t+z_{i}-z_{j}\right)} \\ +\sum_{n=1}^{\infty} \frac{k_{1,2}{ }^{(n-1)}}{\left(2(n-1) t+z_{i}+z_{j}\right)}\end{array}\right]$

$$
\left[\begin{array}{l}
\frac{1}{\left(z_{i}-z_{j}\right)} \\
+\sum_{n=1}^{\infty} \frac{k_{1,2}{ }^{n}}{\left(2 n t+z_{i}-z_{j}\right)}
\end{array}\right.
$$

$$
\left[\begin{array}{l}
\frac{1}{\sqrt{\frac{1}{\lambda^{2}}+\left(z_{i}-z_{j}\right)^{2}}} \\
+\sum_{n=1}^{\infty} \frac{k_{1,2}{ }^{n}}{\sqrt{\frac{1}{\lambda^{2}}+\left(2 n t+z_{i}-z_{j}\right)^{2}}}
\end{array}\right.
$$

(ii) $V_{i j}=\frac{I \rho_{H}}{4 \pi}+\sum_{n=1}^{\infty} \frac{k_{1,2}{ }^{n}}{\sqrt{\frac{1}{\lambda^{2}}+\left(-2 n t+z_{i}+z_{j}\right)^{2}}}$

$$
\left[\begin{array}{l}
+\sum_{n=1}^{\infty} \frac{k_{1,2}{ }^{n}}{\sqrt{\frac{1}{\lambda^{2}}+\left(-2 n t+z_{i}-z_{j}\right)^{2}}} \\
+\sum_{n=1}^{\infty} \frac{k_{1,2}^{(n-1)}}{\sqrt{\frac{1}{\lambda^{2}}+\left(2(n-1) t+z_{i}+z_{j}\right)^{2}}}
\end{array}\right]
$$




$$
\text { (iii) } V_{i j}=\frac{I \rho_{H}}{4 \pi}\left[\begin{array}{l}
\frac{1}{\sqrt{\frac{2}{\lambda^{2}}+\left(z_{i}-z_{j}\right)^{2}}} \\
+\sum_{n=1}^{\infty} \frac{k_{1,2}{ }^{n}}{\sqrt{\frac{2}{\lambda^{2}}+\left(2 n t+z_{i}-z_{j}\right)^{2}}} \\
+\sum_{n=1}^{\infty} \frac{k_{1,2}{ }^{n}}{\sqrt{\frac{2}{\lambda^{2}}+\left(-2 n t+z_{i}+z_{j}\right)^{2}}} \\
+\sum_{n=1}^{\infty} \frac{k_{1,2}{ }^{\infty}}{\sqrt{\frac{2}{\lambda^{2}}+\left(-2 n t+z_{i}-z_{j}\right)^{2}}} \\
k_{1,2}{ }^{(n-1)}
\end{array}\right]
$$

Situation (i) should return $\rho_{H}$ depending only on the thickness of the ice and the coefficient of reflection of the ice/water interface $\left(k_{1,2}\right)$. The thickness of the ice can be obtained from other measurements, and thus the typical thickness of the sea ice being studied can be taken into consideration when using these expressions. The coefficient of reflection $k_{1,2}$ is given by $k_{1,2}=\left(\frac{\rho_{2}-\rho_{1}}{\rho_{2}+\rho_{1}}\right)=\left(\frac{\rho_{s w}-\rho_{m}}{\rho_{s w}+\rho_{m}}\right)$, assuming that for sea ice $\rho_{m}$ is of the order of 100 's of ohmmeters i.e. within the range $100 \Omega \mathrm{m}$ to $1000 \Omega \mathrm{m}$ and that the underlying seawater has $\rho_{s w} \approx 0.4 \Omega \mathrm{m}$, then $k_{1,2}$ will be in the range -0.992 to -0.999 (the value being negative as $\rho_{s w}$ is less than $\rho_{m}$ ). Thus a large change in $\rho_{m}$ has little effect on the value of $k_{1,2}$, and the small range in values means that the resistivity values are only weakly dependent on $k_{1,2}$. As well as the preceding dependencies, situations (ii) and (iii) further depend on the coefficient of anisotropy $(\lambda)$ of the ice, which depends on the resistivity structure and is thus an unknown.

Synthetic data were generated using expressions (2.34) - (2.37) in MATLAB®, for the case of a uniform anisotropic layer with $t=1.4 \mathrm{~m}, \rho_{H}=1000 \Omega \mathrm{m}, \rho_{m}$ in the range $20 \Omega \mathrm{m}$ $\leq \rho_{m} \leq 500 \Omega \mathrm{m}$, and $\lambda$ in the range $0.02 \leq \lambda \leq 0.5$. The anisotropic layer was overlain with a uniform half-space with high resistivity (representing air), and underlain by a uniform half-space with a low resistivity of $0.4 \Omega \mathrm{m}$ (in order to represent the seawater beneath the ice). The datasets each comprised of over 2800 separate electrode 
combinations, which satisfied the restrictions discussed earlier in section 2.2.1. In all cases the summation terms in expressions (2.34) - (2.37) begin to converge after a few hundred terms, such that by $n=500$ subsequent terms in the summations are less than $\mathrm{x} 10^{-5}$ in size. Hence, in creation of synthetic data the infinite summations were truncated to only 500 terms.

Case (i), above, corresponds to the situation of the current and potential electrodes being in the same borehole and thus gives us the $V_{11}$ and $V_{22}$ potentials. The $V_{12}$ and $V_{21}$ potentials can be obtained from cases (ii) or (iii) depending on the borehole configuration used. Recall from section 2.2.1, that if the vertical distance between electrodes is significantly less than the horizontal separation between boreholes, the $V_{12}$ and $V_{21}$ potentials are significantly smaller than the values for $V_{11}$ and $V_{22}$. This can be seen to hold for the three layer case as well, with the difference in the magnitude of the terms from the synthetic dataset, generated with $\rho_{m}=100 \Omega \mathrm{m}$ and $\lambda=0.1$, showing that within the ice the $V_{12}$ and $V_{21}$ terms are around four orders of magnitude smaller than the $V_{11}$ and $V_{22}$ potentials. As such the potential difference is dominated by the potential terms arising from having the current and potential electrode in the same borehole i.e. an expression of the form given above for case (i).

The synthetic dataset, generated with $\rho_{m}=100 \Omega \mathrm{m}$ and $\lambda=0.1$, shows that at most the total of the summation terms in the expression, given in case (i), is $50 \%$ of the value of the first term, which represents the direct path. Thus within the anisotropic middle layer, of the given three layer resistivity structure, the potential measured by this crossborehole technique is between the following values:

$$
\frac{I \rho_{H}}{4 \pi}\left[\frac{0.5}{\left(z_{i}-z_{j}\right)}\right] \leq V_{i j} \leq \frac{I \rho_{H}}{4 \pi}\left[\frac{1.5}{\left(z_{i}-z_{j}\right)}\right]
$$

Comparing with expression (2.2b) $\left(V_{i j}=I \rho / 4 \pi r_{i j}\right)$ for the potential measured in an infinite isotropic medium, it can be seen that even in a bounded anisotropic medium, with the typical thickness and resistivity parameters relevant to sea ice, estimates of the horizontal component of the bulk resistivity should be obtained to within a factor of two of the real value.

The recovered horizontal resistivity structures, obtained from the inversion of the synthetic datasets in RES3DINV, are shown in Figure 2.15 below. From these results it 
can clearly be seen that, despite the approximations in the theoretical development, $\rho_{H}$ is recovered, to within a good degree of accuracy (taking the average value within the ice $\rho_{H}$ is recovered to within $10 \%$ ), for all $\lambda$ values and at most depths within the anisotropic layer representing the sea ice. Only in the layer immediately above the ice/water interface $(1.3 \mathrm{~m}-1.4 \mathrm{~m})$ is there a significant underestimation of $\rho_{H}$. In a field situation the thickness of the anisotropic layer of sea ice is generally known from other measurements. As such, during inversion, the resistivity below this depth can be constrained to a value representative of seawater (as has been applied in the results of Figure 2.15 below).

(a)

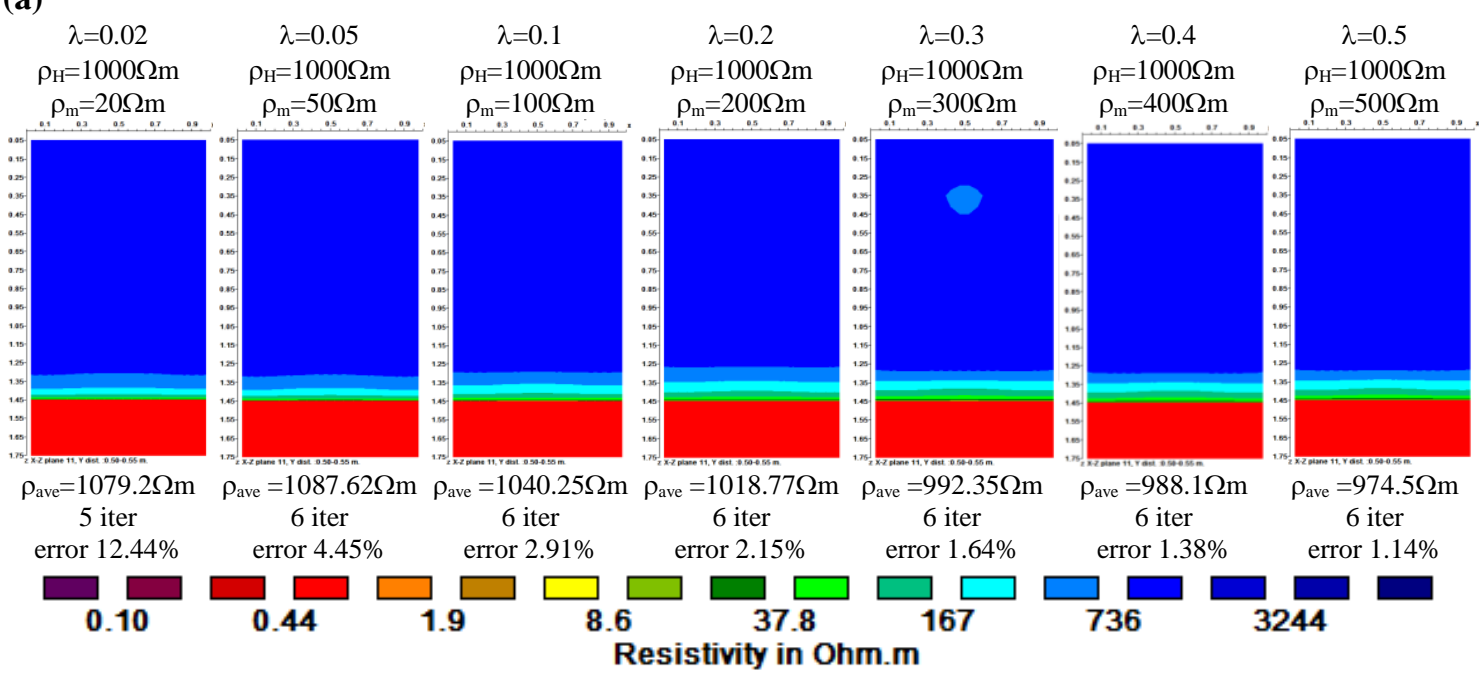

(b)

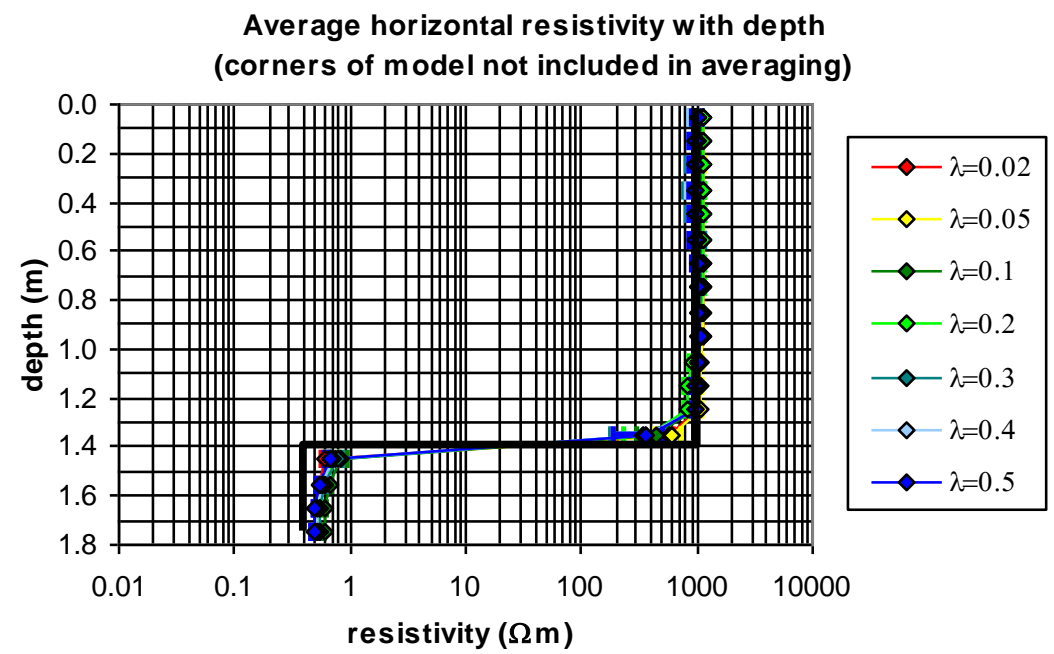

Figure 2.15: Horizontal resistivity recovered from $3 \mathrm{D}$ inversion of synthetic data, for the case of sea ice thickness of $t=1.4 \mathrm{~m}, \rho_{H}=1000 \Omega \mathrm{m}, 20 \Omega \mathrm{m} \leq \rho_{m} \leq 500 \Omega \mathrm{m}, 0.02 \leq \lambda \leq 0.5$, and $\rho_{s w}=0.4 \Omega \mathrm{m}$. (a) Vertical sections through the resistivity structure obtained from RES3DINV. (b) Variation of horizontal resistivity with depth, recovered from the inversion models in (a) by taking the average value at each layer of the model (ignoring the corner cells which are affected by the presence of the boreholes). Given the spatial grid used in the inversion each resistivity represents an average over approximately 400 cells. The true 


\subsubsection{Geometric mean resistivity measurements $\left(\rho_{m}\right)$}

In order to make measurements of the geometric mean component of the resistivity structure either four boreholes or three boreholes with a remote electrode was required. Furthermore it is necessary for the vertical distance between electrodes to be small enough, that it can be assumed that all the electrodes are at the same depth. However, in previous sections it was assumed the ice cover was an infinite medium rather than the more realistic three layer model of sea ice. By applying the required configuration constraints to the relevant expression for the potential in a three layer model (equation (2.34)), it will be possible to determine, in the same way as $\rho_{H}$, whether the resistivity measurements made on sea ice will return $\rho_{m}$ as expected (for an infinite medium) or if the resistivity is dependent upon other factors. The required potential and current electrode combinations have $z_{i}-z_{j} \sim 0$ and either (i) $\left|x_{i}-x_{j}\right|^{2}+\left|y_{i}-y_{j}\right|^{2}=1$ when the electrodes are along a side of a $1 \mathrm{~m}$ by $1 \mathrm{~m}$ square or (ii) $\left|x_{i}-x_{j}\right|^{2}+\left|y_{i}-y_{j}\right|^{2}=2$ when the electrodes are along a diagonal of a $1 \mathrm{~m}$ by $1 \mathrm{~m}$ square. For these two different cases equation (2.34) becomes:

$$
\text { (i) } V_{i j}=\frac{I \rho_{m}}{4 \pi}\left[\begin{array}{l}
1+\sum_{n=1}^{\infty} \frac{k_{1,2}{ }^{n}}{\sqrt{1+\lambda^{2}(2 n t)^{2}}} \\
+\sum_{n=1}^{\infty} \frac{k_{1,2}{ }^{n}}{\sqrt{1+\lambda^{2}\left(-2 n t+2 z_{j}\right)^{2}}} \\
+\sum_{n=1}^{\infty} \frac{k_{1,2}{ }^{n}}{\sqrt{1+\lambda^{2}(-2 n t)^{2}}} \\
\text { (ii) } V_{i j}=\frac{I \rho_{m}}{4 \pi}\left[\begin{array}{l}
\sum_{n=1}^{\infty} \frac{k_{1,2}{ }^{(n-1)}}{\sqrt{1+\lambda^{2}\left(2(n-1) t+2 z_{j}\right)^{2}}} \\
+\sum_{n=1}^{\infty} \frac{k_{1,2}{ }^{n}}{\sqrt{2}}+\frac{k_{1,2}{ }^{\infty}}{\sqrt{2+\lambda^{2}\left(-2 n t+2 z_{j}\right)^{2}}} \\
+\sum_{n=1}^{\infty} \frac{k_{1,2}{ }^{n}}{\sqrt{2+\lambda^{2}(-2 n t)^{2}}} \\
+\sum_{n=1}^{\infty} \frac{k_{1,2}{ }^{(n-1)}}{\sqrt{2+\lambda^{2}\left(2(n-1) t+2 z_{j}\right)^{2}}}
\end{array}\right]
\end{array}\right.
$$


In both of the situations the potential measured is dependent on coefficient of anisotropy $(\lambda)$, the coefficient of reflection of the ice/water interface $\left(k_{1,2}\right)$ and the thickness of the ice cover. The thickness of the ice can be obtained from other measurements and is thus a known, whereas $\lambda$ depends on the resistivity structure and is thus unknown. In section 2.3.2.1 it was shown that the resistivity values are only weakly dependent on $k_{1,2}$. Hence, the value obtained for the geometric mean resistivity, during the inversion of the measurements, is dependent on $\lambda$ and does not return the true $\rho_{m}$ value.

Once again synthetic data were generated using expressions (2.34) - (2.37) in MATLAB $®$, this time for the case of a uniform anisotropic layer with $t=1.4 \mathrm{~m}, \rho_{m}=$ $100 \Omega \mathrm{m}$, values of $\rho_{H}$ in the range $200 \Omega \mathrm{m} \leq \rho_{H} \leq 5000 \Omega \mathrm{m}$, and values of $\lambda$ in the range $0.02 \leq \lambda \leq 0.5$. Again, the anisotropic medium was overlain with a uniform half-space with high resistivity (representing air), and underlain by a uniform half-space with a low resistivity of $0.4 \Omega \mathrm{m}$ (in order to represent the seawater beneath the ice). The datasets comprised of over 800 separate electrode combinations, which satisfied the restrictions discussed earlier in section 2.2.2.

For the synthetic dataset generated with $\rho_{m}=100 \Omega \mathrm{m}$ and $\lambda=0.1$, the total of the summation terms in both case (i) and (ii) is of the same order of magnitude as the direct term but negative. Using these results the expressions for the potentials in case (i) and (ii) above can be compared with expression (2.2b) $\left(V_{i j}=I \rho / 4 \pi r_{i j}\right)$ for the potential measured in an infinite isotropic medium. This indicates that in a bounded anisotropic medium, with the typical thickness and resistivity parameters relevant to sea ice, estimates of the geometric mean component of the bulk resistivity will likely be underestimated.

The recovered geometric mean resistivity structures, obtained from the inversion of the synthetic datasets in RES3DINV, are shown in Figure 2.16 below. As with the horizontal resistivity, measurements of the thickness of the anisotropic layer of sea ice can be made in the field. As such, during inversion, the resistivity below this depth can be constrained to a value representative of seawater (as has been applied in the results of Figure 2.16 below).From the inversions it can clearly be seen that, given the approximations in the theoretical development, the resistivities obtained from the inversion $\left(\rho_{m}^{M}\right)$ do not accurately recover the true resistivity $\left(\rho_{m}\right)$. In fact the geometric mean component of the bulk resistivity of sea ice is underestimated. Even for $\lambda=0.5$ the 
recovered $\rho_{m}$ is only $70 \%$ of the true value, in the upper $1 \mathrm{~m}$ of the anisotropic medium. Closer to the ice-water interface the recovered value drops to about $40 \%$ of the true value. This underestimation increases with decreasing $\lambda$, so that by the time $\lambda<0.2$ the recovered $\rho_{m}$ is of the order of only $1 \%$ of the true value and has a more complicated variation with depth. The thinner the ice cover the more severe the underestimation, for instance at a thickness of $0.85 \mathrm{~m}$, for $\lambda=0.5$, the recovered $\rho_{m}$ is only $30 \%$ of the true value

(a)

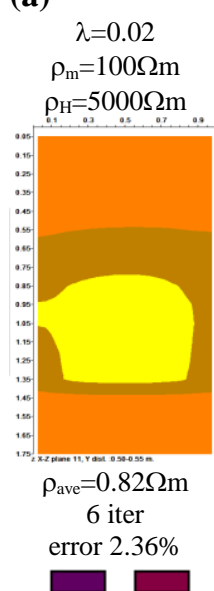

$$
\begin{gathered}
\lambda=0.05 \\
\rho_{\mathrm{m}}=100 \Omega \mathrm{m}
\end{gathered}
$$

$\rho_{\mathrm{H}}=2000 \Omega \mathrm{m}$

$\lambda=0.1$

$\rho_{\mathrm{m}}=100 \Omega \mathrm{m}$

$\rho_{\mathrm{H}}=1000 \Omega \mathrm{m}$
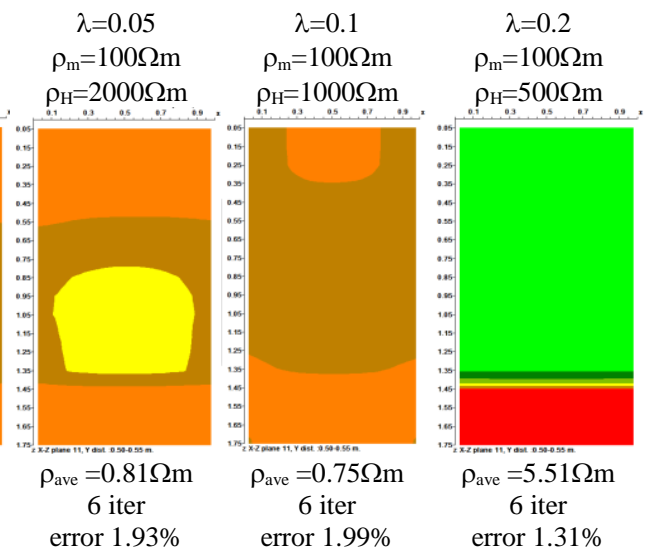

$\lambda=0.3$
$\rho_{\mathrm{m}}=100 \Omega \mathrm{m}$
$\rho_{\mathrm{H}}=333.33 \Omega \mathrm{m}$

$\lambda=0.4$

$\rho_{\mathrm{m}}=100 \Omega \mathrm{m}$

$\rho_{\mathrm{H}}=250 \Omega \mathrm{m}$
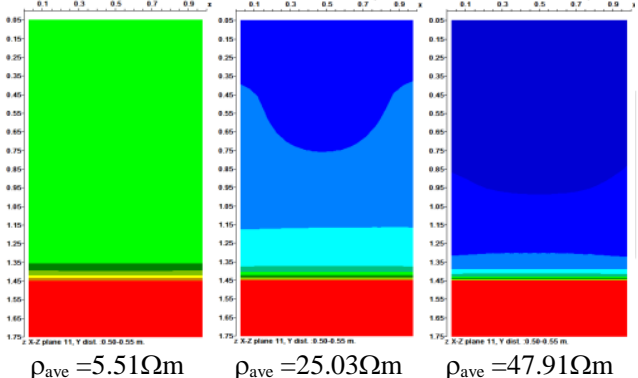

$\lambda=0.5$

$\rho_{\mathrm{m}}=100 \Omega \mathrm{m}$ $\rho_{\mathrm{H}}=200 \Omega \mathrm{m}$

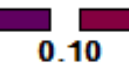

error $1.93 \%$ error $1.99 \%$

$\rho_{\text {ave }}=5.51 \Omega \mathrm{m}$

$\rho_{\text {ave }}=25.03 \Omega \mathrm{m}$

$\rho_{\text {ave }}=47.91 \Omega \mathrm{m}$

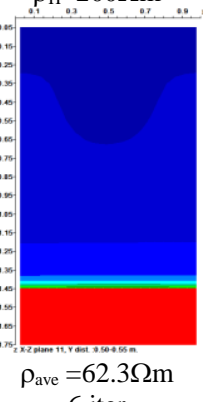

error $1.31 \%$

error $1.26 \%$

4.3

110

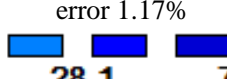

Resistivity in Ohm.m

28.1

(b)

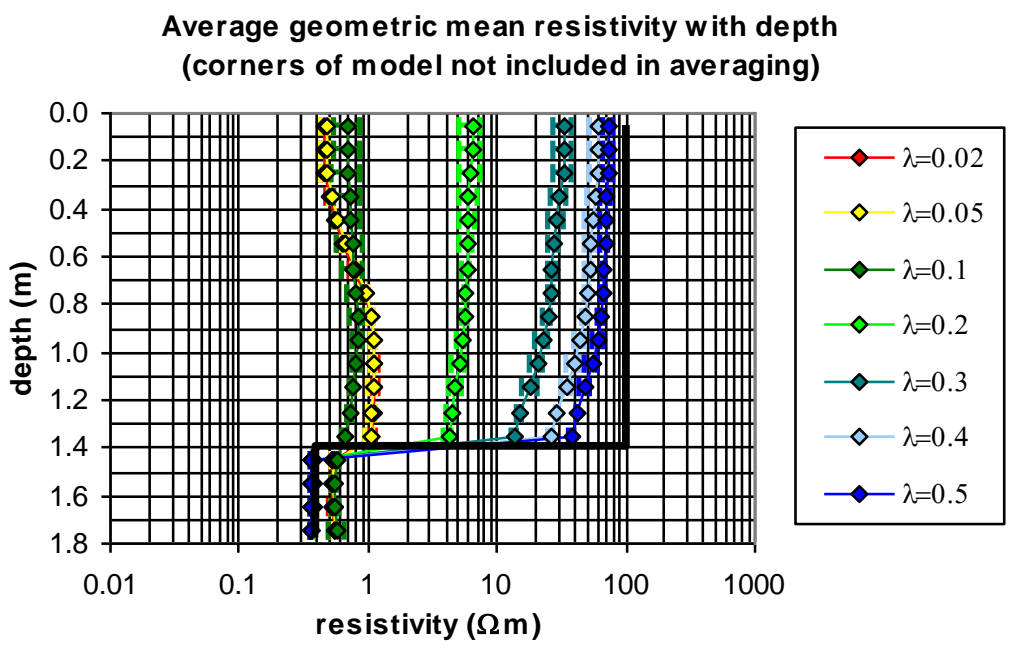

Figure 2.16: Geometric mean resistivity recovered from 3D inversion of synthetic data, for the case of sea ice thickness of $t=1.4 \mathrm{~m}, \rho_{m}=100 \Omega \mathrm{m}, 200 \Omega \mathrm{m} \leq \rho_{H} \leq 5000 \Omega \mathrm{m}, 0.02 \leq \lambda \leq 0.5$, and $\rho_{s w}=0.4 \Omega \mathrm{m}$. (a) Vertical sections through the resistivity structure obtained from RES3DINV. (b) Variation of geometric mean resistivity with depth, recovered from the inversion models in (a) by taking the average value at each layer of the model (ignoring the corner cells which are affected by the presence of the boreholes).

Given the spatial grid used in the inversion each resistivity represents an average over approximately 400 cells. The true value of $\rho_{m}$ is shown by the solid line. 
Clearly, once sea ice is considered as a bounded anisotropic medium, obtaining reliable measurements of the geometric mean resistivity through DC cross-borehole tomography is not straightforward. However, inversion of synthetic datasets (as mentioned above), which recover model resistivity values $\left(\rho_{m}^{M}\right)$, makes it possible to derive an empirical parameterization of the relationship between the true value of $\rho_{m}$ and the recovered value $\rho_{m}^{M}$. For a given thickness of ice that relationship is given by the following expression.

$$
\rho_{m}^{M}=F(\lambda) \rho_{m}
$$

where $F(\lambda)$ is a polynomial in the anisotropy coefficient, the form of which is determined from inversion of synthetic data sets. Synthetic data sets are created for a uniform block of ice of the given thickness, a given $\rho_{m}$ and varying values of $\lambda$. Inversion of the synthetic data provides average $\rho_{m}^{M}$ values for each layer within the models. These values are divided by the true resistivity $\left(\rho_{m}\right)$, given to the created ice cover, and plotted against the $\lambda$ value used, thus obtaining a polynomial in $\lambda$ for each layer of the inversion models.

Using (2.6) and (2.7) equation (2.38) may be expressed as

$$
\rho_{m}^{M}=\lambda F(\lambda) \rho_{H}
$$

As demonstrated above $\rho_{H}$ can be determined from inversion of real data, the form of $F(\lambda)$ can be deduced from inversion of synthetic data, generated for ice of the same thickness as that the measured data was obtained from, and $\rho_{m}^{M}$ is also obtained from inversion of the measured or real data. Thus a value for $\lambda$ can be found from numerical solution of (2.39), for each layer in the resulting 3D inversions of the measured data. An estimate of the actual value of $\rho_{m}$, as a function of depth, can then be determined from (2.38).

As an example, for sea ice with a thickness of $1.4 \mathrm{~m}$ the polynomial in the anisotropy coefficient in the depth range $0.5 \mathrm{~m}-0.6 \mathrm{~m}$ can be represented by

$$
F(\lambda)=-57.411 \lambda^{4}+47.077 \lambda^{3}-7.1312 \lambda^{2}+0.3452 \lambda
$$

for $\lambda \leq 0.5$.

For the specific case of $\lambda=0.1$ the inversion of the synthetic data yields a value of $\rho_{m}^{M}$ $=0.748 \Omega \mathrm{m}$ in this depth range. As the created ice, used to generate the synthetic data, 
has a true geometric mean value of $100 \Omega \mathrm{m}$ and the case being considered uses a coefficient of anisotropy of 0.1 the horizontal resistivity of the ice is $1000 \Omega \mathrm{m}$. Inserting these values for $\rho_{m}^{M}$ and $\rho_{H}$ into expression (2.39) the equation can be numerically solved to return a value of 0.113 for $\lambda$. This value is close to the true value of 0.1 used in the generation of the synthetic data sets. Furthermore inserting the value found for lambda and the $\rho_{m}^{M}$ value into (2.38) returns a value of $\rho_{m}=113 \Omega \mathrm{m}$ for the true geometric mean value of the created ice. This value is close to the value of $\rho_{m}=100 \Omega \mathrm{m}$ assigned to the created ice and is significantly better than the value returned by the inversion of the synthetic data set.

There remain a number of complicating factors when applying this to a real situation. These include (i) the structure of the real sea ice may include a variation in $\lambda$ with depth which is not considered when creating the synthetic datasets; (ii) the ice-water interface has been modelled as a sharp boundary, whereas in nature this may not be the case; (iii) the reliance on the results of $3 \mathrm{D}$ inversions, both of real and synthetic data, may well introduce problems of resolution of the resistivity structure; and (iv) even in the inversions of synthetic data it is clear (Figure 2.16) that the derived values of $\rho_{m}^{M}$ are relatively insensitive to $\lambda \leq 0.1$. Quantifying the uncertainty in derived values of $\rho_{m}$ resulting from these factors is non-trivial, but as a consequence it is likely when interpreting values derived in the described manner, one should bear in mind that these may only be correct to within a factor of the order of perhaps 3-4.

\subsubsection{Surface resistivity measurements}

If the current $(C)$ and potential $(P)$ electrodes are at the air/ice interface of the three layer model i.e. $z_{j}=z_{i}=0$ (see Figure 2.11 above) then the ray paths that can travel between $C$ and $P$ are limited. There is still a direct path from $C$ to $P$ and an infinite series representing the potential due to images corresponding to ray paths reflected an even or odd number of times, the first reflection being from the lower boundary (as in Figure 2.8 and Figure 2.9). However, as the electrodes are at the surface there can no longer be ray paths with their first reflection in the upper boundary (as in Figure 2.10). Hence, these terms can be removed from the expression of the total potential measured at $P$. Furthermore, recall from 2.1.1, that when making surface (rather than buried) measurements the geometric term $4 \pi$ becomes $2 \pi$. Using these modifications equation (2.34) can be rewritten to give an expression for the total potential measured at $P$ when 
the current $(C)$ and potential $(P)$ electrodes are on the surface of anisotropic sea ice cover.

$$
V_{i j}=\frac{I \rho_{m}}{2 \pi}\left(\begin{array}{c}
\frac{1}{\sqrt{\left|x_{i}-x_{j}\right|^{2}+\left|y_{i}-y_{j}\right|^{2}}} \\
+\sum_{n=1}^{\infty} \frac{k_{1,2}{ }^{n}}{\sqrt{\left|x_{i}-x_{j}\right|^{2}+\left|y_{i}-y_{j}\right|^{2}+\lambda^{2}|2 n t|^{2}}} \\
+\sum_{n=1}^{\infty} \frac{k_{1,2}{ }^{n}}{\sqrt{\left|x_{i}-x_{j}\right|^{2}+\left|y_{i}-y_{j}\right|^{2}+\lambda^{2}|-2 n t|^{2}}}
\end{array}\right)
$$

This expression can also be obtained by simply substituting $z_{i}=z_{j}=0$ into equation (2.34) and simplifying.

From expression (2.40) it can be seen that the resistivity value obtained from the inversion of surface soundings is dependent upon the coefficient of anisotropy $(\lambda)$, the coefficient of reflection of the ice/water interface $\left(k_{1,2}\right)$ and the thickness of the ice cover. The thickness of the ice can be obtained from other measurements and is thus a known, whereas $\lambda$ depends on the resistivity structure and is thus unknown. In section 2.3.2.1 it was shown that the resistivity values are only weakly dependent on $k_{1,2}$. As such the resistivities obtained are dependent on $\lambda$ and are not the true $\rho_{m}$ values.

Synthetic data was generated using expression (2.40) in MATLAB®, for the case of a uniform anisotropic layer with $t=1.4 \mathrm{~m}, \rho_{m}=100 \Omega \mathrm{m}, 200 \Omega \mathrm{m} \leq \rho_{H} \leq 5000 \Omega \mathrm{m}$ and 0.02 $\leq \lambda \leq 0.5$, overlain with a uniform half-space with high resistivity (representing air), and underlain by a uniform half-space with an low resistivity of $0.4 \Omega \mathrm{m}$ (in order to represent the seawater beneath the ice). The dataset comprised of 11 electrode combinations obtained using a Wenner array configuration with electrode separations of $0.1,0.2,0.3,0.4,0.6,0.8,1.0,1.5,2,3$ and 4 meters.

For the synthetic dataset generated with $\rho_{m}=100 \Omega \mathrm{m}$ and $\lambda=0.1$, the total of the summation terms in expression (2.40) is of the magnitude of between one half to one times the direct term but negative. Using these results expression (2.40) for the potential above can be compared with expression (2.2a) $\left(V_{i j}=I \rho / 2 \pi r_{i j}\right)$ for the potential 
measured on the surface of an infinite isotropic medium. This suggests that surface soundings on a bounded anisotropic medium, provide measurements that underestimate the geometric mean component of the bulk resistivity. The size of the total of the summation terms increases with the increase in the electrode separation, this would indicate that the underestimation should worsen with depth.

The recovered geometric mean resistivity structures, obtained from the inversion of the synthetic datasets, are shown in Figure 2.17 below. From the inversions it can clearly be seen that the retrieved resistivity structure is not an accurate representation of the true structure, though it is more complicated than a straight over or under-estimation.

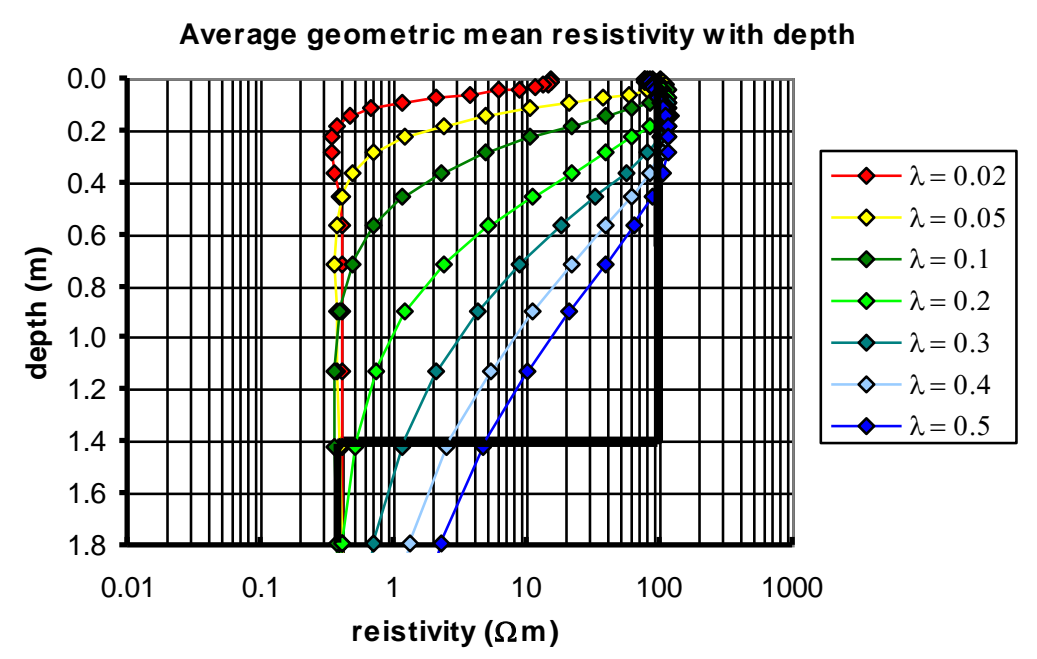

Figure 2.17: Variation of geometric mean resistivity with depth, recovered from 1D inversion of synthetic surface resistivity data. The true value of $\rho_{m}$ is shown by the solid line.

The major issue with inversions obtained from surface soundings is that the depth data is incorrect. Thus the interpretation of surface resistivity soundings, in terms of variation of geometric mean resistivity with depth, is complex. This makes comparison between the retrieved and true values difficult, as it is uncertain as to where the ice/sea interface occurs in the retrieved resistivity structure. From Figure 2.17 it seems that with low $\lambda$ values the resistivity structure returned from the inversion of the data shows only the resistivity structure around the water/ice interface. As the $\lambda$ value increases more of the resistivity structure of the sea ice itself is revealed. It has been suggested that although the depth data are incorrect, the geometric mean resistivity structure can be obtained from surface soundings if the resistivity structure obtained from inversion is stretched to match similar features observed in the $\rho_{H}$ inversion sections. 


\subsubsection{Summary}

Although more complicated than making measurements on a simplistic infinite anisotropic medium, it has been shown above that estimates of the horizontal and geometric mean resistivity of a bounded structure representative of sea ice can be obtained from cross-borehole measurement methods. Theoretically the use of crossborehole data, derived from appropriate combinations of electrodes, allows direct measurement of the horizontal component of the bulk resistivity of sea ice (or other bounded anisotropic medium within which $\left.\rho_{V}<\rho_{H}\right)$. Furthermore, although the particular structure of sea ice means that exact measurements of the geometric mean resistivity cannot be obtained, it is possible to derive a reasonable estimate of $\rho_{m}$ from cross-borehole measurements via numerical modelling. As with the infinite medium the vertical component of the bulk resistivity, which cannot be directly measured, can be obtained from these horizontal and vertical resistivities (see equation (2.7) section 2.1.2) using the following relationship

$$
\rho_{m}=\sqrt{\rho_{V} \rho_{H}} \Rightarrow \rho_{v}=\rho_{m}{ }^{2} / \rho_{H}
$$

Thus it will be possible to study a complete 3D model of the anisotropic resistivity structure of sea ice and how it varies over the melt season, using DC resistivity crossborehole tomography measurements. 


\section{Chapter 3 \\ Field Work}

\subsection{Arctic site - Barrow, Alaska (2008)}

In April - June 2008 cross-borehole electrical resistivity measurements were made in first-year landfast sea ice in the Chukchi Sea, approximately $1 \mathrm{~km}$ off the coast of Barrow, Alaska (Figure 3.1).

Barrow is located 515km above the Arctic Circle (http://en.wikipedia.org/wiki/Barrow, Alaska) on the Chukchi Sea coast of Alaska, and as such is the northernmost community in the USA. The community is traditionally known as Ukpeagvik, "place where snowy owls are hunted". Barrow takes its modern name from Point Barrow, named in 1825 for Sir John Barrow of the British Admiralty, during plotting of the Arctic coastline of North America by Captain Beechey of the Royal Navy. During the 1940s and 1950s, the military played an influential role in the area and during this time, the Naval Arctic Research Lab (NARL) was built near Barrow (http://www.cityof barrow.org).

At the time of the measurements the Barrow Arctic Science Consortium (BASC) was a not-for-profit organization, operating from NARL, that was dedicated to the encouragement of research and educational activities pertaining to Alaska's North Slope and the adjacent portions of the Arctic Ocean. The BASC was organized in 1995 as a way for three local organizations; (i) The North Slope Borough (the regional government for Alaska's North Slope), (ii) The Ukpeagvik Iñupiat Corporation (a corporation owned by the Native people of Barrow, founded under authority of the Alaska Native Claims Settlement Act), (iii) Ilisagvik College (the local centre for postsecondary education) and other interested persons to work together in support of Arctic science. BASC provided the logistical support for the Arctic measurements with NARL acting as a home base (http://www.arcticscience.org/).

In Barrow the sun does not set between May 10th and August 2nd each summer, and does not rise between November 18th and January 24th each winter. The climate of Barrow features light annual precipitation, averaging 5 ' $(12.7 \mathrm{~cm})$ with annual snowfall of $20^{\prime \prime}(50.8 \mathrm{~cm})$. The temperature ranges from $-56{ }^{\circ} \mathrm{F}\left(-48.9^{\circ} \mathrm{C}\right)$ to $78{ }^{\circ} \mathrm{F}\left(25.6^{\circ} \mathrm{C}\right)$ and 
the daily minimum temperature is below freezing 324 days of the year (http://www.dce d.state.ak.us/dca/commdb/CF_BLOCK.cfm). February is the coldest month with average temperatures of $-16{ }^{\circ} \mathrm{F}\left(-26.7^{\circ} \mathrm{C}\right)$ (average from between 1971 and 2000) and July the hottest with an average of $40.4^{\circ} \mathrm{F}\left(4.7^{\circ} \mathrm{C}\right)$ (http://www.nws.noaa.gov/climate /xmacis.php?wfo=pafg). The Chukchi Sea is typically ice-free from mid-June through October (http://www.dced.state.ak.us/dca/commdb/CF_BLOCK.cfm).

Dr Malcolm Ingham, Dr Daniel Pringle and I made six sets of horizontal and geometric mean resistivity measurements, these were obtained at roughly one to two weekly intervals over the 3 month measurement period (April - June, 2008). Surface resistivity soundings were also made, on four different occasions, using a Wenner array configuration. The University of Alaska Fairbanks (UAF) sea ice mass balance site was also operated in this same area $\left(71^{\circ} 21^{\prime} 56.45^{\prime}, \mathrm{N}, 156^{\circ} 32^{\prime} 39.01^{\prime \prime} \mathrm{W}\right)$ from $7^{\text {th }}$ February to $17^{\text {th }}$ June, recording snow and ice thickness, sea level, relative humidity and air, ice and water temperatures (http://seaice.alaska.edu/gi/observatories/ barrow_sealevel, Druckenmiller et al., 2009). Standard measurements were also carried out on sea ice cores in order to determine salinity profiles.

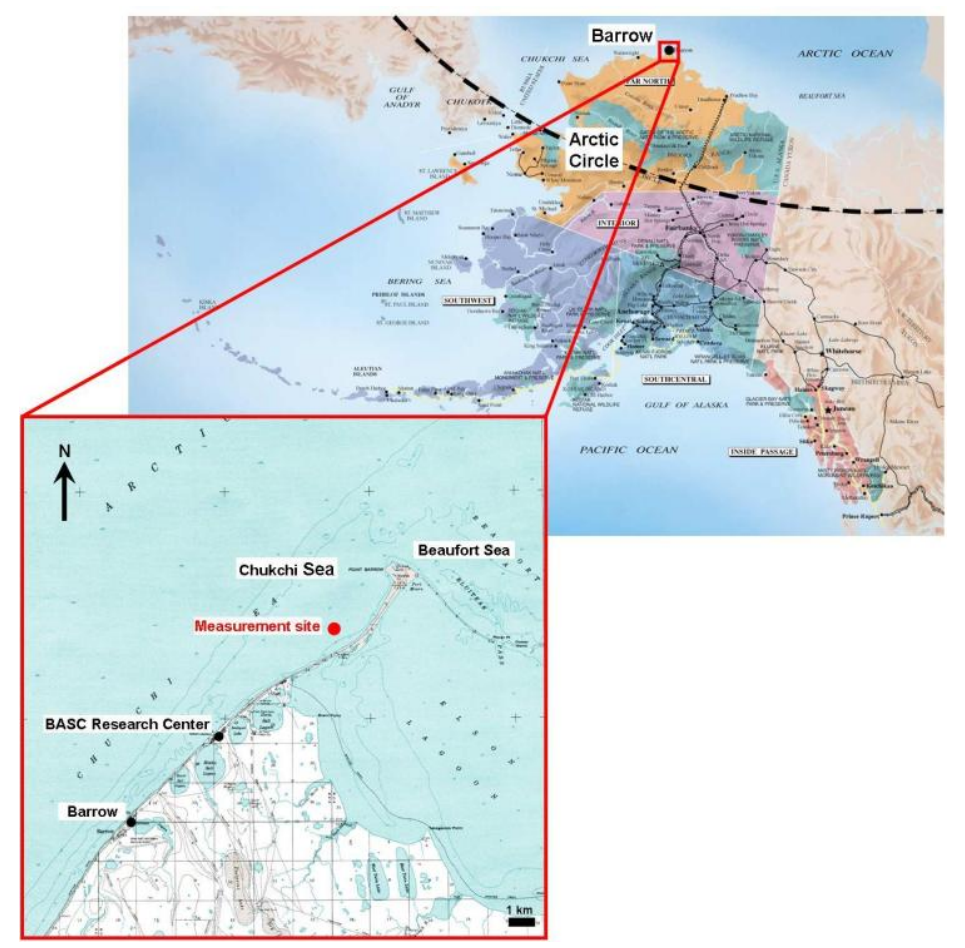

Figure 3.1: Maps showing the approximate location of the measurement site, in first-year sea ice $\sim 1 \mathrm{~km}$ off the coast of Barrow, Alaska. The Alaskan map is from http://mappery.com/map-of/Alaska-Map-5 and the Barrow insert is a subregion of Barrow (B-4), Alaska 1955 - USGS 1:63,360 map for point Barrow area, available from http://store.usgs.gov/b2c_usgs/usgs/maplocator/(xcm=r3standardpitrex_prd\&layout =6_1_61_48\&uiarea=2\&ctype $=$ areaDetails $\&$ carea $=\% 24$ ROOT $) /$. do. 


\subsection{Antarctic site - McMurdo sound, Ross Dependency (2009)}

In November 2009 cross-borehole electrical resistivity measurements were made in McMurdo Sound in first-year sea ice, formed as an extension of Ross Ice Shelf, at a site approximately $10 \mathrm{~km}$ off the coast of Ross Island, Antarctica (Figure 3.2).

Ross Island is located off the coast of Victoria Land, Antarctica in McMurdo Sound at a latitude of $77^{\circ} 40^{\prime} \mathrm{S}$, within the Ross Dependency. In 1841 Sir James Clark Ross discovered Victoria Land and the Ross Sea, including Ross Island (later named in honour of him by Robert F. Scott) and claimed them for Britain. The British Government defined its current borders in 1923 and entrusted it to the administration of New Zealand (http://en.wikipedia.org/wiki/Ross_Dependency).

Ross Island has an area of 2,460 $\mathrm{km}^{2}$ (http://en.wikipedia.org/wiki/Ross_Island) and is home to Scott Base, New Zealand's Antarctic research station, located on Pram point $3 \mathrm{kms}$ from the US McMurdo Station. The proposal for a New Zealand base in Antarctica was put to the New Zealand Government by the Antarctic Society in 1953. The building of the base began in 1956 to support the Trans-Antarctic Expedition and International Geophysical Year of 1956-1959 (http://www.antarcticanz.govt.nz/scottbase/brief-history). Field operations and events logistics at Scott Base are overseen by Antarctica New Zealand, a Crown Entity established in 1996, responsible for developing, managing and executing New Zealand Government activities in Antarctica and the Southern Ocean (http://www.antarcticanz.govt.nz/about-antarctica-nz).

Antarctica is the driest, coldest, windiest continent on Earth. For inland Antarctica temperatures rise to about $-30^{\circ} \mathrm{C}$ in summer and fall to below $-80^{\circ} \mathrm{C}$ in winter. However, at Scott Base the average temperature ranges annually from around $-29.7^{\circ} \mathrm{C}$ to $-4.5^{\circ} \mathrm{C}$, with January being the warmest month and August the coldest month (http://www.niwa.co.nz/education-and-training/schools/resources/climate/antarctic).

Most precipitation in the Antarctic falls as snow or ice crystals, although during summer rain can occur near the coast. It is estimated (windy conditions make it difficult to accurately measure snowfall) that the annual snowfall over the continent is equal to about $150 \mathrm{~mm}$ of water. In the elevated inland it is much drier than this, while near the coast there is more precipitation (http://www.antarcticanz.govt.nz/scott-base/weather). 
At Scott Base, the sun sets in late April and does not rise again until the end of August (http://nztabs.ictar.aq/rossscott.php).

McMurdo Sound is about $55 \mathrm{~km}$ long and similarly wide, with the western shoreline defined by Victoria Land, the eastern boundary by Ross Island and the southern end by McMurdo Ice Shelf (a region of the Ross Ice Shelf). During the winter McMurdo Sound presents a virtually impenetrable expanse of surface ice. Even during summer, ships approaching McMurdo Sound are often blocked by various concentrations of ice (http://en.wiki pedia.org/wiki/McMurdo_Sound).

Dr Malcolm Ingham and I took four sets of horizontal and geometric mean resistivity data using borehole measurements at intervals of roughly one to two days. These measurements were accompanied by surface soundings made using a Wenner array. A mass balance site was operated at the same site $\left(77^{\circ} 46^{\prime} 33.12^{\prime \prime} \mathrm{S}, 166^{\circ} 18^{\prime} 46.26^{\prime \prime} \mathrm{E}\right)$ as part of Antarctica New Zealand science event K131, providing measurements of temperature, ice thickness, snow depth and observations of platelet ice. As at Barrow salinity data were obtained from ice cores.

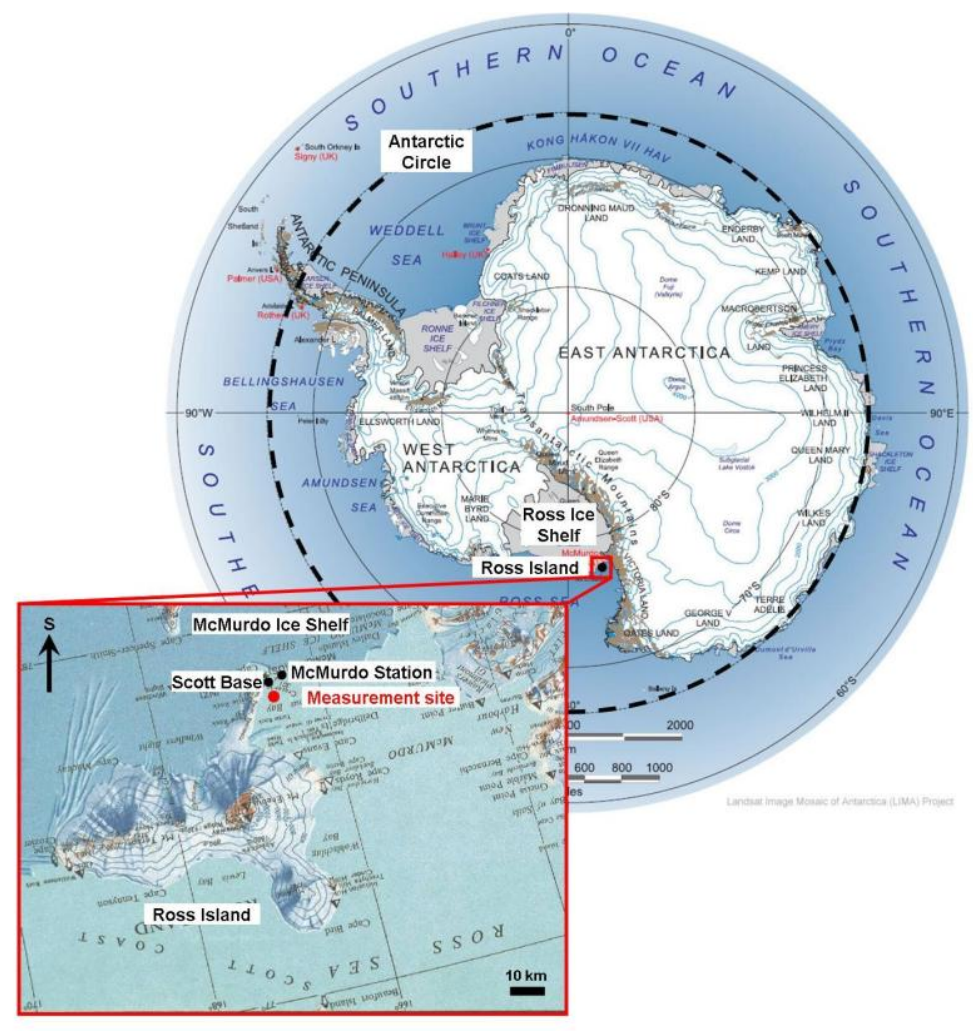

Figure 3.2: Maps showing the approximate location of the measurement site, in first-year sea ice $\sim 10 \mathrm{~km}$ off the coast of Ross Island, Antarctica. The Antarctica map is from http://lima.nasa.gov/antarctica/ and the Ross Island insert is from http://www.linz.govt.nz/topography/topo-maps/antarctica/ross-searegions.jpg. 


\subsection{Resistivity measurements}

The electrode strings used in the resistivity measurements (Figure 3.3) were constructed at Victoria University of Wellington (VUW), and consisted of 18 electrodes of $5 \mathrm{~cm} \mathrm{x}$ $5 \mathrm{~cm}$ squares (or $5 \mathrm{~cm}$ diameter circles) of marine-grade stainless steel washers spaced at intervals of $0.1 \mathrm{~m}$ along a multi-core cable, held in place by cable clamps. Takeouts from the cable are bolted and soldered onto the corner of each electrode. Holes are drilled in the other corners of the electrodes to provide better electrical contact as seawater refreezes around the electrode strings.
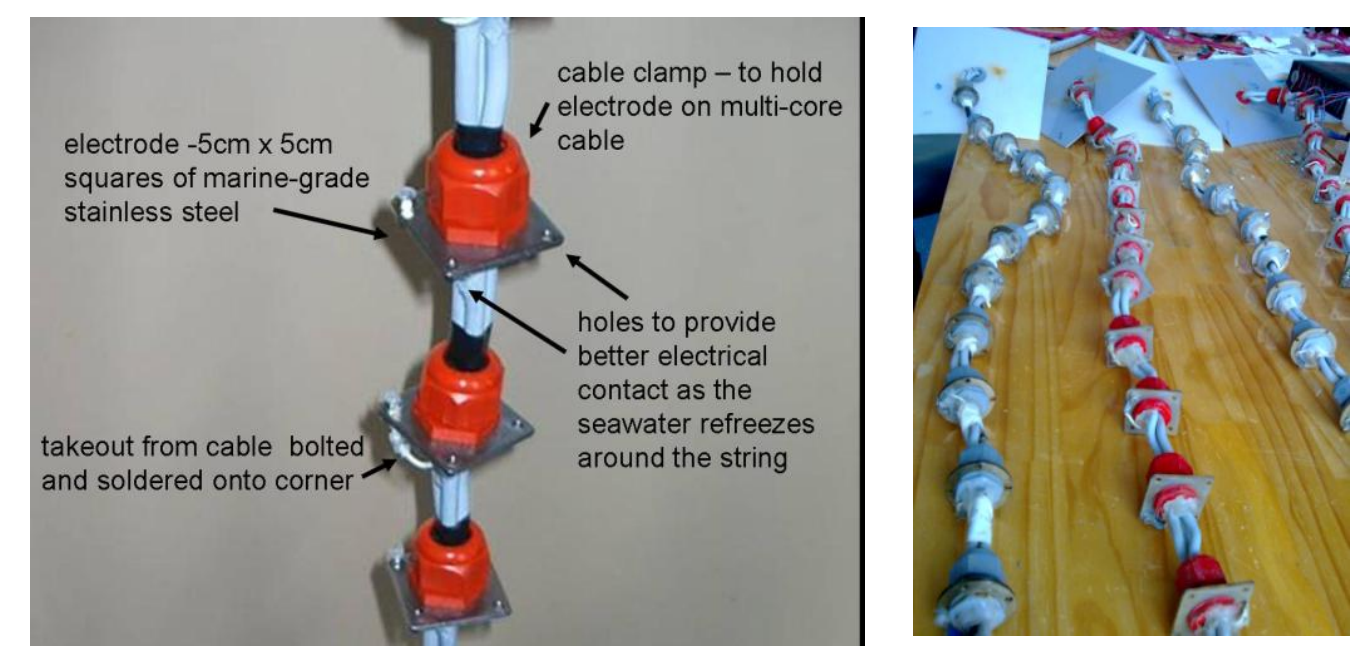

Figure 3.3: Photos of the electrode strings used to make the cross-borehole resistivity measurements.

Early in the growth of the ice the electrode strings were positioned into boreholes drilled in thin first year sea ice. A solid plate held the string in place relative to the surface of the ice and weights suspended from the end kept it vertical as the electrodes were progressively frozen in as the ice thickened. Four strings were deployed at the corners of a $1 \mathrm{~m} \times 1 \mathrm{~m}$ square, allowing measurements to be made through the undisturbed sea ice between boreholes. This reduces the sensitivity to any atypical ice formed around the electrodes as the seawater in the boreholes refreeze.

At the surface the electrode strings are connected, via cables, to a banana plug board consisting of four rows, each with enough plugs for all of the electrodes in a single borehole. Depending on the measurements being made and the electrode arrangement required the necessary plugs are connected to a set of 'smart' electrodes. During the Arctic field work only 16 'smart' electrodes were available, but this doubled to 32 for 
the Antarctic field work. Having more 'smart' electrodes available leads to a more automated measurement procedure, ideally there would be as many 'smart' electrodes as there are electrodes in the boreholes. The 'smart' electrodes can be programmed, through a central control instrument, to make automated measurements along the entire depth of the borehole using set electrode configurations, without manually moving the plugs. However, as there are only 16 or 32 'smart' electrodes and 72 electrodes in the four boreholes, it is necessary to occasionally move the plugs to the next group of electrodes to make a complete set of measurements. In this way measurements are made which cover the depth of the boreholes. The 'smart' electrodes are controlled by a GF Instruments, s.r.o ARES datalogger, which measures the voltage and current - values that can then be used to find the apparent resistance of the sea ice. The equipment setup in the field, during the Arctic measurements can be seen in Figure 3.4 below. The same configuration was used in the Antarctic, except there were two more boxes of 'smart' electrodes that could be connected for any given measurement set.

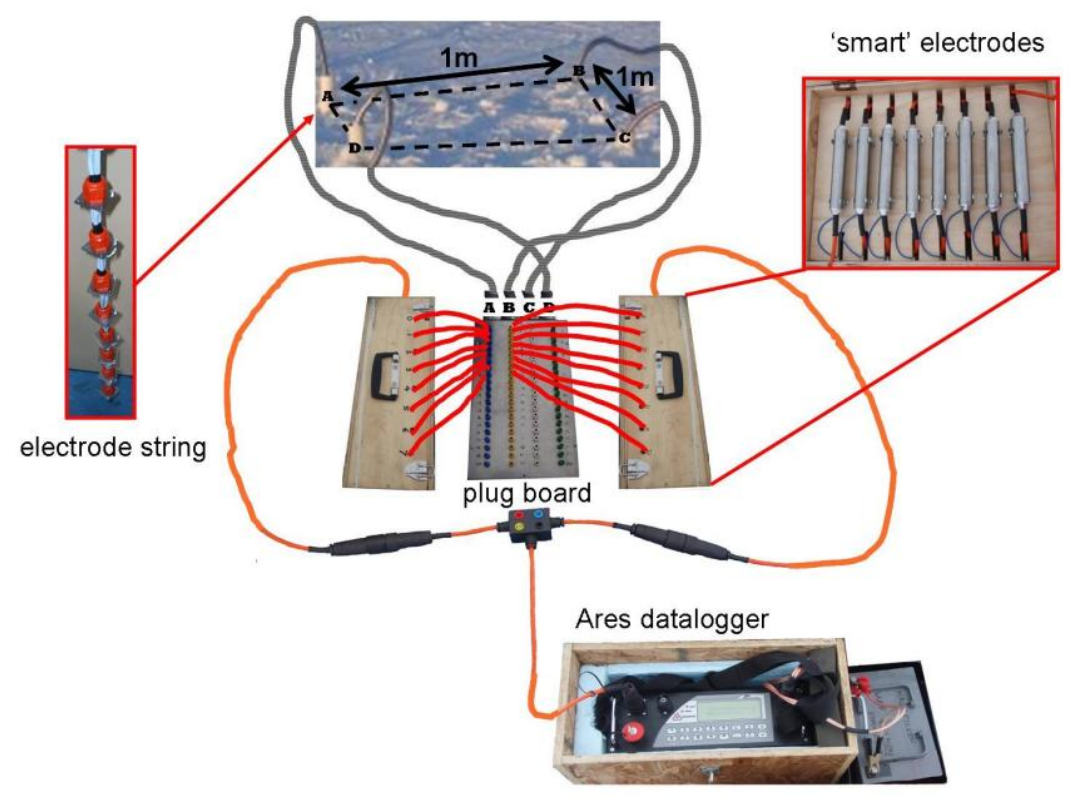

Figure 3.4: Field setup of equipment for cross-borehole resistivity measurements in the Arctic.

In order to determine the horizontal resistivity, measurements were made between each of the six borehole pairs, using appropriate electrode arrangements (as given in section 2.2.1). 3D inversion of these data allow a reasonable estimate of $\rho_{H}$ to be derived for the volume contained by the boreholes. Similarly measurements, aimed at determining $\rho_{m}$ were obtained, this time using appropriated electrode arrangements between four 
boreholes (see section 2.2.2). Inversion of this data set yields a geometric mean resistivity, which is an underestimate of the true value, but can be used to retrieve $\rho_{m}$ through numerical modelling (see section 2.3.2.2).

Surface soundings were made using screws inserted into the surface of the ice in a Wenner array configuration, with electrode spacing of $0.1,0.2,0.3,0.4,0.6,0.8,1.0$, 1.5, 2.0, 3.0 and 4.0 metres. Estimates of the geometric mean resistivity can be obtained from the surface soundings. However, depth information is incorrect making interpretation of the results complex (as indicated in section 2.3.2.3).

\subsubsection{Horizontal resistivity measurements}

Measurements of the horizontal resistivity are made between each of the six borehole pairs $(\mathrm{AB}, \mathrm{BC}, \mathrm{CD}, \mathrm{AD}, \mathrm{AC}, \mathrm{BD})$, as in Figure 3.5. Each measurement involves one current and one potential electrode in each borehole, arranged so that the potential difference measured will provide an approximation to the horizontal resistivity (see section 2.2.1). The depth of the measurement set and which electrodes are used as the current or potential electrodes is determined by positioning of the plugs and programming of the 'smart' electrodes.

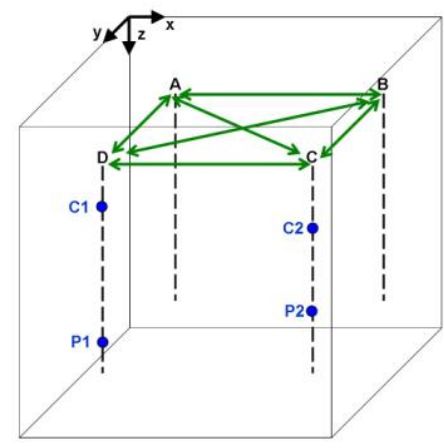

Figure 3.5: Borehole setup for horizontal resistivity measurements, measurements are made between each of the six pairs of boreholes as indicated by the green arrows.

For measurements of the horizontal resistivity in the Arctic there are two different plug configurations and measurement programs used. The first, labelled rhoh1 measurements, involves connecting the equipment as shown in Figure 3.6 below for measurements between boreholes A and B. Initially electrodes 1-8 in each borehole are 
used, then electrodes 6-13 and 9-16. This leads to there being three measurement sets, consisting of 90 measurements, for one borehole pair, therefore over all six pairings $(\mathrm{AB}, \mathrm{BC}, \mathrm{CD}, \mathrm{AD}, \mathrm{AC}, \mathrm{BD})$ there are 18 measurement sets with 1,620 measurements in total.

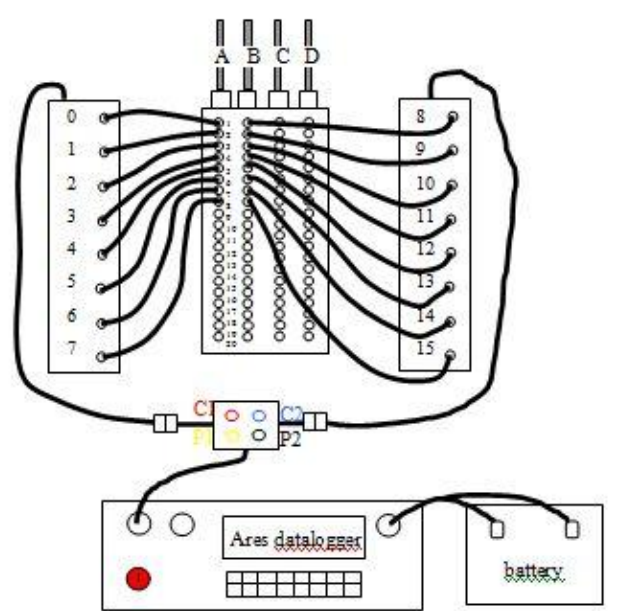

(a)

\begin{tabular}{|ccccc|}
\hline 0 & 8 & 1 & 9 & GEN \\
0 & 8 & 2 & 10 & GEN \\
1 & 9 & 0 & 8 & GEN \\
2 & 10 & 0 & 8 & GEN \\
0 & 9 & 1 & 10 & GEN \\
0 & 10 & 1 & 11 & GEN \\
\hline
\end{tabular}

(c)
A $\quad$ B

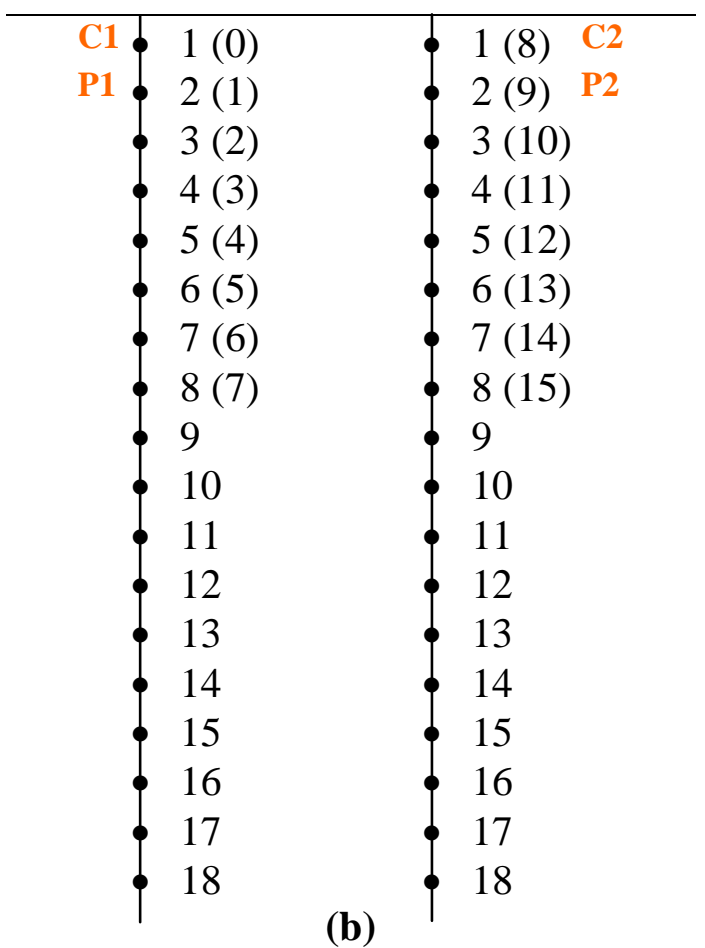

Figure 3.6: Example of plug and ARES datalogger setup for Arctic field measurements of the horizontal resistivity, labelled rhoh1. Example shows measurements between the upper eight electrodes of boreholes A and B (a) plug setup connecting 'smart' electrodes with the electrode strings in the borehole (b) electrode strings in borehole - indicating which electrodes are being used (c) first few lines of the program determining the measurements made.

Figure 3.6(c) displays the first few lines of the programming file used to control the 'smart' electrodes. The first line indicates that electrode 0 of the 'smart' electrodes (or electrode 1 in borehole $\mathrm{A}$ ) is set to be the current electrode $\mathrm{C} 1$, electrode 8 (electrode 1 in borehole $\mathrm{B}$ ) set to $\mathrm{C} 2$, electrode 1 ( 2 in $\mathrm{A}$ ) set to $\mathrm{P} 1$ and 9 ( 2 in $\mathrm{B}$ ) to $\mathrm{P} 2$, as is shown in Figure 3.6(b). The 'GEN' at the end of the line simply means that this configuration of electrodes is moved down the borehole, i.e. each electrode is incremented so that the next set of measurements will be $1(\mathrm{C} 1), 9(\mathrm{C} 2), 2(\mathrm{P} 1)$ and $10(\mathrm{P} 2)$ and so on until the end of the connected smart electrodes is reached with 6 (C1), $14(\mathrm{C} 2), 7$ (P1) and 15 
(P2). The program will then move on to the next line and make measurements for that electrode configuration.

The second plug and program configuration, labelled as rhoh2, involves making some of the same measurements as with the first option but reversing the polarity by connecting the 'smart electrodes $0-7$ to the electrodes in borehole $\mathrm{B}$ and 8-15 to borehole A as shown in Figure 3.6 below. Initially electrodes 1-8 in each borehole are used, then electrodes 6-13 and 9-16. This leads to there being three measurement sets, consisting of 64 measurements, for one borehole pair, therefore over all six pairings $(\mathrm{AB}, \mathrm{BC}, \mathrm{CD}, \mathrm{AD}, \mathrm{AC}, \mathrm{BD})$ there are 18 measurement sets with 1,152 measurements in total.

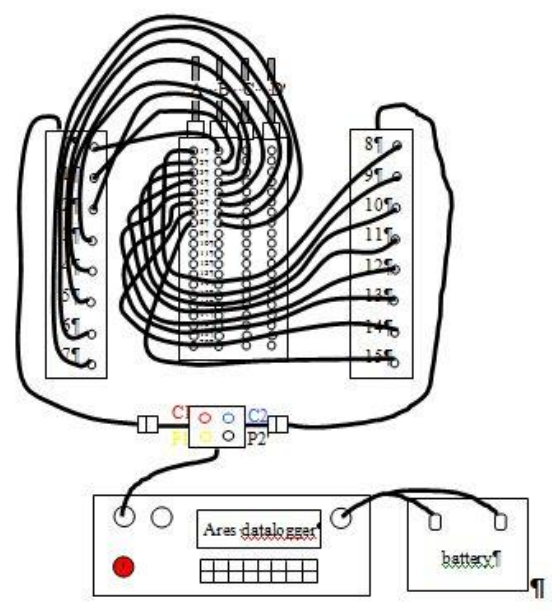

(a)

\begin{tabular}{|ccccc|}
\hline 0 & 9 & 1 & 10 & GEN \\
0 & 10 & 1 & 11 & GEN \\
1 & 10 & 0 & 9 & GEN \\
1 & 11 & 0 & 10 & GEN \\
0 & 11 & 1 & 12 & GEN \\
0 & 12 & 1 & 13 & GEN \\
\hline
\end{tabular}

(c)
A

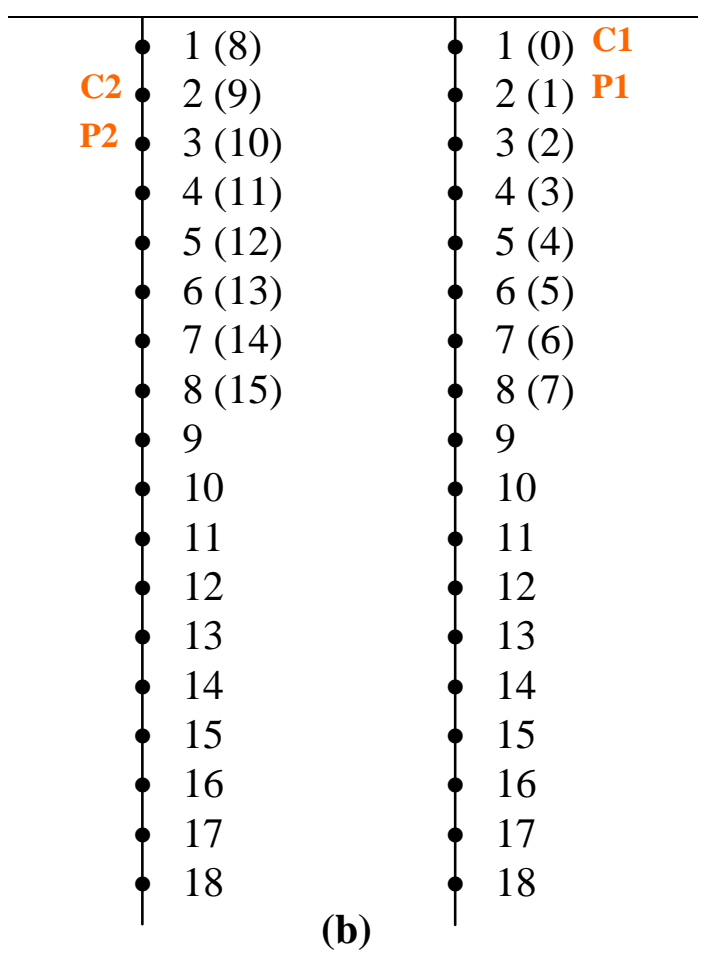

Figure 3.7: Example of plug and ARES datalogger setup for Arctic field measurements of the horizontal resistivity, labelled rhoh2. Example shows measurements between the upper eight electrodes of boreholes A and B (a) plug setup connecting 'smart' electrodes with the electrode strings in the borehole (b) electrode strings in borehole - indicating which electrodes are being used (c) first few lines of the program determining the measurements made.

Using both these program and plug configurations to measure the horizontal resistivity should give a total of 2,772 data points, before processing. 
The ARES datalogger, used to make these measurements, encountered problems when both current electrodes were in the seawater under the ice. In this situation the datalogger detects too low a contact resistance and no measurement is made with that electrode configuration. This arises as the ARES tries to optimise the potential difference reading at $5 \mathrm{mV}$, and when both current electrodes are in the water this optimisation means that the datalogger requires a current which is larger than can be supplied. Thus for the lower electrodes all measurements need to be made manually using an older instrument, a terrameter SAS 300C datalogger developed by ABEM Instrument $\mathrm{AB}$, which allows the magnitude of the current (rather than the potential) to be set.

In 2009 there were 32 'smart' electrodes available for measurements made in the Antarctic. A similar field setup to that used in the Arctic (Figure 3.4) was employed here, except there are now two joined boxes of 'smart' electrodes connected to each connector of the $\mathrm{T}$ junction. These extra 16 'smart' electrodes allowed a more automated measurement process with only one change of plugs needed to make measurements down the entire depth of a borehole pair. For this measurement period the program files rhoh1 and rhoh2, used in the Arctic, were combined into rhoh1, and made 539 measurements over electrodes 1-16 for each borehole pair (on the first Antarctic measurement set two separate files were still being used). An additional program file, rhoh3 (the first data set uses two separate files, rhoh3 and rhoh4, one for each of the two different polarities), made 118 measurements which involved the two remaining electrodes (17 and 18) in each borehole pair. In the Antarctic it was possible to use the ARES datalogger and automated system for these lower electrodes, as the ice thickness was greater than the length of the electrode strings and no electrodes were in the seawater below. Over the six borehole pairs and the two program files 3,942 data points were collected before processing.

\subsubsection{Geometric mean resistivity measurements}

The geometric mean resistivity is obtained from two different measurement configurations (Figure 3.8); one using a four electrode array with each of the electrodes at approximately the same depth in separate boreholes, while the second places three electrodes at the same depth in separate boreholes and a surface remote electrode 
approximately 10 meters away. Initially, in the Arctic measurements $\left(7^{\text {th }}\right.$ Apr to $26^{\text {th }}$ Apr) the four electrode measurements were made with all the electrodes at the same depth, but later ( $8^{\text {th }}$ May to $16^{\text {th }}$ Jun) this constraint was lessen to having the electrodes at approximately the same depth, this approximation is shown as acceptable in section 2.2.2. When using the three electrode array it was found that having a current electrode as the remote electrode lead to the potential differences generally being very small and to a high current being applied, which caused melting of the ice around the remote electrode. When the electrode was moved closer to the boreholes $(2 \mathrm{~m})$ the measurements appeared to be more sensible. However at this distance the infinite electrode assumption that the remote electrode should be positioned at a distance 20 times the maximum separation of $\mathrm{C} 1$ and $\mathrm{P} 1$ may no longer be valid. The minimum separation between P1 and C1 was $0.1 \mathrm{~m}$ which means the remote electrode should be $2 \mathrm{~m}$ or further away in order for the infinite electrode assumptions to hold for these measurement sets. This electrode configuration affected measurements made from the $7^{\text {th }}$ Apr to the $26^{\text {th }}$ Apr. From the $20^{\text {th }}$ May measurements were made using a potential electrode as the remote electrode, which removes the problem with high current. It can easily be shown theoretically, that using a potential electrode as the remote electrode yields the same measurements as a remote current electrode. In the Antarctic the later measurement methods were used; electrodes were at approximately the same depth and a potential electrode was used as the remote electrode.

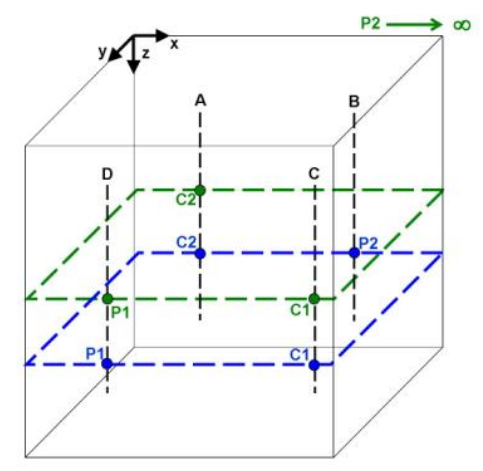

Figure 3.8: Borehole setups for geometric mean resistivity measurements.

For measurements of the geometric mean resistivity in the Arctic there are two different plug configurations and measurement programs used. The first, labelled rhomean1, involves using all four boreholes and connecting the equipment as shown in Figure 3.9 below. Initially electrodes 1-4 in each borehole are used, then electrodes 5-8, 9-12 and 
13-16. This leads to there being four measurement sets, consisting of 90 measurements, therefore in total there are 360 measurements.

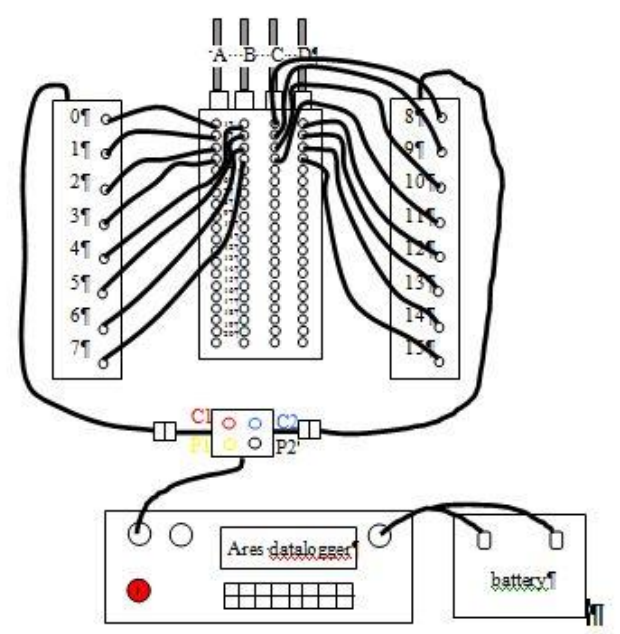

(a)

\begin{tabular}{|lcccc|}
\hline 0 & 4 & 12 & 8 & GEN \\
4 & 8 & 0 & 12 & GEN \\
8 & 12 & 4 & 0 & GEN \\
12 & 0 & 8 & 4 & GEN \\
0 & 8 & 4 & 12 & GEN \\
4 & 12 & 0 & 8 & GEN \\
\hline
\end{tabular}

(c)
A $\quad$ B $\quad$ C $\quad$ D

\begin{tabular}{|c|c|c|c|}
\hline $1(0)$ & \&1(4)C & $1(8)$ & $\mathbf{P} 2 \nmid 1(12) \mathbf{P} 1$ \\
\hline $2(1)$ & $\{2(5)$ & $2(9)$ & 2 (13) \\
\hline $3(2)$ & $3(6)$ & $3(10)$ & $3(14)$ \\
\hline $4(3)$ & $4(7)$ & $4(11)$ & $4(15)$ \\
\hline 5 & 5 & 5 & 5 \\
\hline 6 & 6 & 6 & 6 \\
\hline 7 & 7 & 7 & 7 \\
\hline 8 & 8 & 8 & 8 \\
\hline 9 & $\{9$ & 9 & 9 \\
\hline 10 & $\{10$ & 10 & 10 \\
\hline 11 & $\{11$ & 11 & 11 \\
\hline 12 & 12 & 12 & 12 \\
\hline 13 & 13 & 13 & 13 \\
\hline 14 & 14 & 14 & 14 \\
\hline 15 & 15 & 15 & 15 \\
\hline 16 & 16 & 16 & 16 \\
\hline 17 & 17 & 17 & 17 \\
\hline 18 & $\{18$ & 18 & 18 \\
\hline
\end{tabular}

Figure 3.9: Example of plug and ARES datalogger setup for Arctic field measurements of the geometric mean resistivity, labelled rhomean1. (a) plug setup connecting 'smart' electrodes with the electrode strings in the borehole (b) electrode strings in borehole - indicating which electrodes are being used (c) first few lines of the program determining the measurements made.

As with the horizontal measurements, Figure 3.9(c) displays the first few lines of the programming file used to control the 'smart' electrodes. The red line indicates that electrode 0 of the 'smart' electrodes (or electrode 1 in borehole A) is set to be the current electrode $\mathrm{C} 1$, electrode 4 (electrode 1 in borehole B) set to $\mathrm{C} 2$, electrode 12 (1 in D) set to P1 and 8 (1 in C) to P2, as is shown in Figure 3.9(b). The measurements then continue down the boreholes until the end of the connected smart electrodes is reached with $3(\mathrm{C} 1), 7(\mathrm{C} 2), 15(\mathrm{P} 1)$ and $11(\mathrm{P} 2)$. The program will then move on to the next line and make measurements for that electrode configuration.

The second plug and program configuration, labelled as rhomean2 (see Figure 3.10), is similar to that of rhomean1 with connections to four electrodes in each borehole. However the black terminal on the T-box is connected to a remote potential electrode fixed into the surface of the ice about 10m away in line with the A and B boreholes. In 
the early stages measurements were made with the $\mathrm{C} 2$ (blue terminal on the T-box) as the remote electrode. Initially electrodes 1-4 in each borehole are used, then electrodes 5-8, 9-12 and 13-16. This leads to there being four measurement sets, consisting of 90 measurements, therefore in total there are 360 measurements.

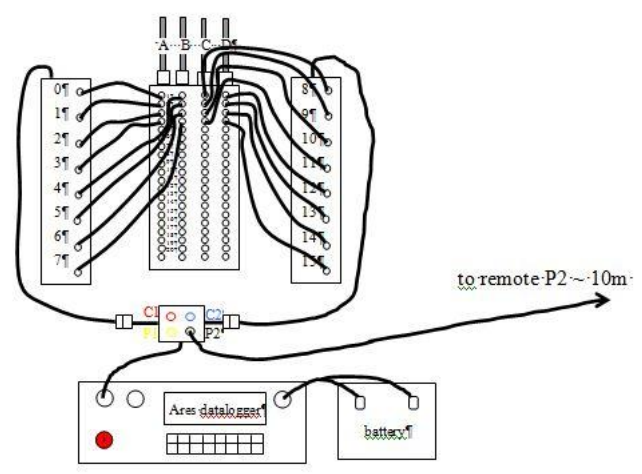

(a)

\begin{tabular}{|ccccc|}
\hline 0 & 4 & 12 & -1 & GEN \\
0 & 8 & 12 & -1 & GEN \\
0 & 4 & 8 & -1 & GEN \\
4 & 8 & 12 & -1 & GEN \\
0 & 12 & 4 & -1 & GEN \\
0 & 12 & 8 & -1 & GEN \\
\hline
\end{tabular}

(c)
A $\quad$ B $\quad$ C $\quad$ D

\begin{tabular}{|c|c|c|c|}
\hline $1(0) C$ & 1 $1(4)$ & $1(8)$ & 1 (12) P1 \\
\hline $2(1)$ & $2(5)$ & $2(9)$ & $2(13)$ \\
\hline $3(2)$ & $3(6)$ & $3(10)$ & $3(14)$ \\
\hline $4(3)$ & $4(7)$ & $4(11)$ & $4(15)$ \\
\hline 5 & 5 & 5 & 5 \\
\hline 6 & 6 & 6 & 6 \\
\hline 7 & 7 & 7 & 7 \\
\hline 8 & 8 & $\{8$ & 8 \\
\hline 9 & 9 & 9 & 9 \\
\hline 10 & 10 & 10 & 10 \\
\hline 11 & 11 & 11 & 11 \\
\hline 12 & 12 & 12 & 12 \\
\hline 13 & 13 & 13 & 13 \\
\hline 14 & 14 & 14 & 14 \\
\hline 15 & 15 & 15 & 15 \\
\hline 16 & 16 & 16 & 16 \\
\hline 17 & 17 & 17 & 17 \\
\hline 18 & 18 & 18 & 18 \\
\hline
\end{tabular}

Figure 3.10: Example of plug and ARES datalogger setup for Arctic field measurements of the geometric mean resistivity, labelled rhomean2. (a) plug setup connecting 'smart' electrodes with the electrode strings in the borehole (b) electrode strings in borehole - indicating which electrodes are being used (c) first few lines of the program determining the measurements made.

Figure 3.10(c) displays the first few lines of the programming file used to control the 'smart' electrodes, the -1 's indicate that this electrode is not used, in this case P2.

Using both these program and plug configurations to measure the geometric mean resistivity should give us a total of 720 measurements. This is significantly less than the number of measurements made for the horizontal resistivity because of the need to use all four boreholes when making a measurement of the geometric mean resistivity.

Since there were 32 'smart' electrodes available for measurements made in 2009 in the Antarctic, measurements could be made over a block of eight, rather than only four, electrodes. Once again this allowed a more automated measurement process with only 
two changes of plugs needed to make measurements down the entire depth of the boreholes. For this measurement period the program files rhomean1 and rhomean2, used in the Arctic, were combined into rhom1, and made 435 measurements over electrodes 1-8 and then the same over electrodes 9-16 (on the first set of measurements made in the Antarctic two separate files were still being used). An additional program file, rhom3 (the first data set uses two separate files, rhom3 and rhom4, one for each of the two different measurement configurations), made 154 measurements which included the two remaining electrodes (17 and 18) in each borehole. In total the two program files allowed 1,024 measurements to be taken in the field.

Table 3.1 and Table 3.2, shown below, give a summary of the measurements made in both the Arctic and Antarctic. 


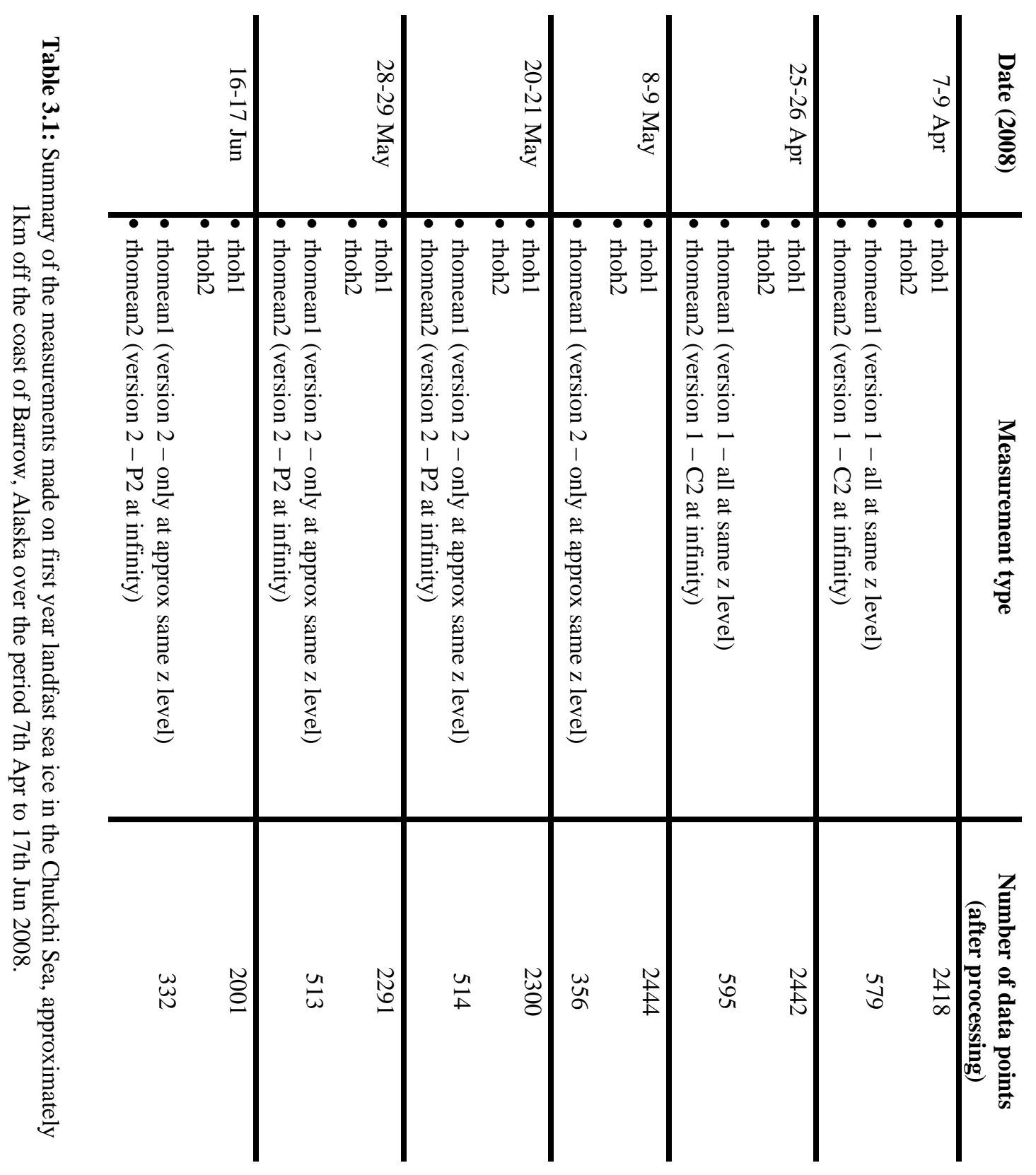




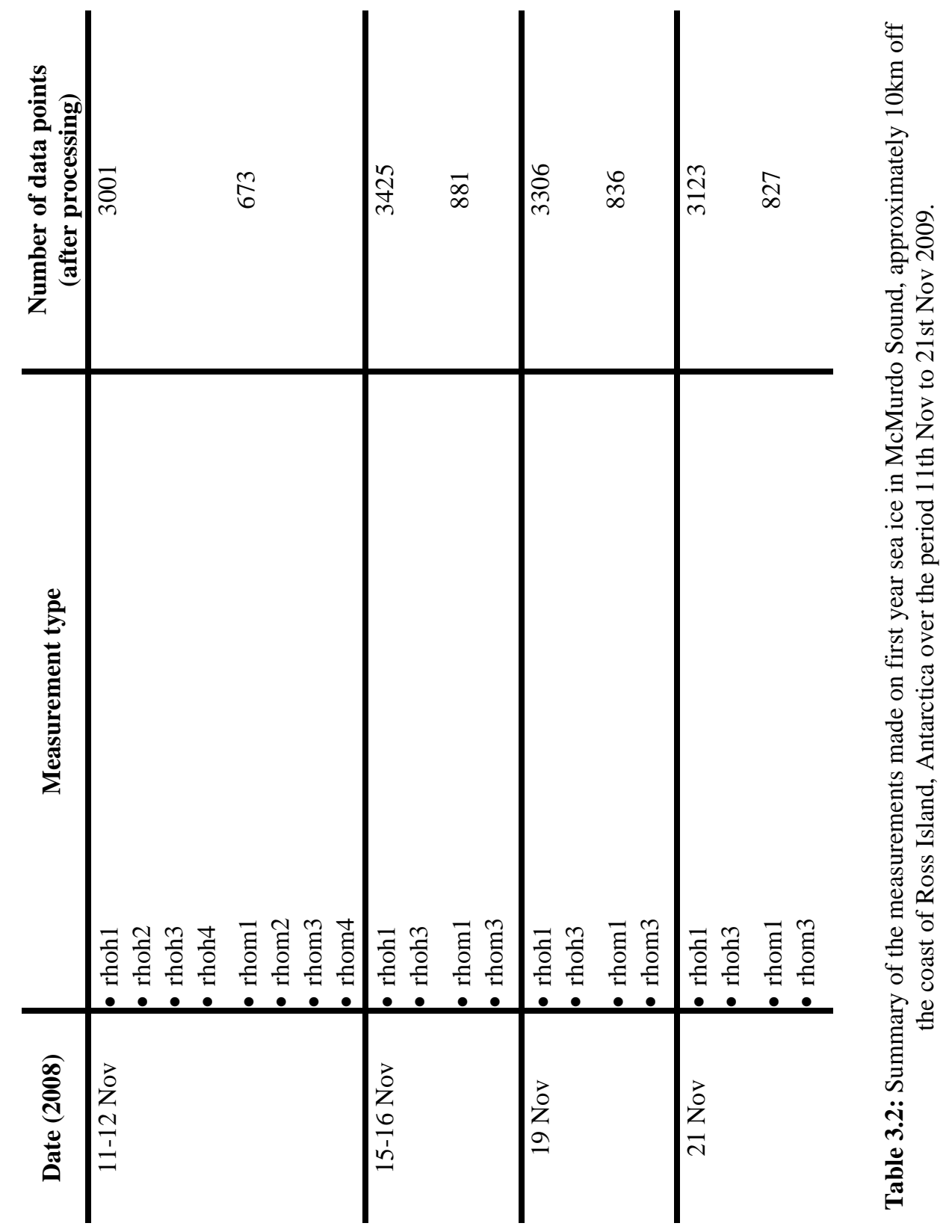




\subsubsection{Dataloggers}

The principal datalogger used in the field was a GF Instruments, s.r.o ARES datalogger. It contained the programs used to control the 'smart' electrodes and stored the data as measurements were made down the boreholes. The ARES datalogger can be used with the following measurement methods (i) self potential measurements - measurements of naturally occurring electrical potentials (SP), (ii) resistivity profiling (RP), (iii) vertical electrode sounding (VES) and (iv) 2D/3D multi-electrode cable survey (2D multicable). As we are using multiple cables of smart electrodes and taking 3D data, option (iv) was used for our measurements.

The ARES datalogger requires specifications of the measurement area. This involves the location of measurement profile in the measurement area and the spacing and length of the electrode strings. The profile is a number identifying the profile, the $\boldsymbol{x}$-loc and $\boldsymbol{y}$ loc give the position of the first electrode and direction defines the direction of measurement along the profile - as shown in Figure 3.11 below.

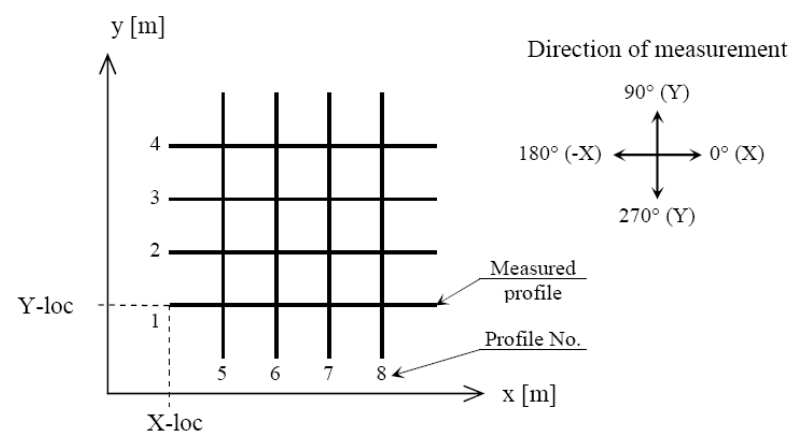

Figure 3.11: Diagram from ARES user manual showing the specification of the profile position.

The datalogger assumes surface profiles with the electrode strings laid out along the surface of the measurement medium, whereas our measurements are borehole measurements. Thus the setup of the location of the measurement profile is somewhat arbitrary. Length is the total length of the measured profile and distance is the electrode spacing. Using the 'smart' electrodes in the Arctic, the instrument is told that there are 16 electrodes attached and that they are all $5 \mathrm{~m}$ apart, hence giving a length of (16-1) x 5 $=75 \mathrm{~m}$. Whereas in the Antarctic there are 32 'smart' electrodes at distances of $5 \mathrm{~m}$ giving a length of (32-1) x $5=155 \mathrm{~m}$. 
Since the geometry of the actual electrode strings is different from that given to the datalogger the calculation of the apparent resistivity in the data files must be ignored, instead focusing on the voltage and current values that can be used to calculate the resistance values of each electrode combination. Using these resistances and correct borehole geometry the inversion program Res $3 \operatorname{dinv}^{\mathrm{TM}}$ produced by Geotomo software (see Chapter 4) is able to calculate the correct resistivities.

The different measurement programs are stored on the datalogger and the one required for the current measurement set is selected from a list. The datalogger then requires the size of the potential to be set. The potential was set to the lowest option $(5 \mathrm{mV})$ in order to attempt to keep the current as small as possible when the resistance is low. As mentioned above problems occur when both current electrodes are in the seawater, the datalogger detects the electrodes as having too low a contact resistance and no measurement is made (even with the low potential of $5 \mathrm{mV}$ set). In order to make measurements with both the current electrodes in the seawater a Terrameter SAS 300C (by $\mathrm{ABEM}$ Instrument $\mathrm{AB}$ ) was used as it allows the size of the current to be set.

The ARES datalogger was set to make four measurements for each data point, with the maximum standard deviation set to the default that appeared on the screen $(2 \%)$. If the standard deviation of the data point is higher than the set maximum the measurements for that point are repeated.

Once the required program and smallest potential had been selected the ARES datalogger displays screens with the number of layers and data points, an estimation of the time of the measurement set and information on electrode connection and use. Next there are options for the position of any infinite or dummy electrodes. As our measurements of the geometric mean resistivity involve a surface remote electrode one electrode is set to be an infinite electrode with the $\mathrm{x}$ location inserted as $150 \mathrm{~m}$ and the $\mathrm{y}$ location 0m. Dummy electrodes are inserted if for some reason there are electrodes that are not to be used. During the Arctic measurements a break occurred in the connection to electrode 5 in borehole $\mathrm{d}$. In order to stop the datalogger attempting to make measurements with a disconnected electrode this electrode was entered as a dummy electrode. 
Measurements can now be initialised and the datalogger will display warning messages to notify if there are electrodes with very high resistances. Figure 3.12 below gives an example of a data file obtained from the datalogger after measurement.

Device:ARES-G4 v4.7, SN: 0701146

Locality:mbs

Operator:

Date: $4 / 8 / 2008$

Note:

Profile: 1

$\mathrm{X}-\mathrm{loc}: 0 \mathrm{~m}$

Y-loc:0m

Direction:0 (X)

Length:75m

Distance:5m

MC-set: rhoh1

S-min:5m

S-max:75m

Pulse:0.5s

IP-Windows:

$\begin{array}{cccccccccc}\begin{array}{c}\text { C1 } \\ {[\mathrm{el}]}\end{array} & \text { C2 } & \text { P1 } & \text { P2 } & & & & \text { AppRes } & \text { St-dev } \\ 0 & 8 & 1 & 9 & \text { GEN } & 30.48 & 5027.66 & 19.14 & 2967.54 & 0.4 \\ 1 & 9 & 2 & 10 & \text { GEN } & 33.08 & 5027.7 & 19.16 & 2734.31 & 0.5 \\ 2 & 10 & 3 & 11 & \text { GEN } & 34.05 & 5027.71 & 19.15 & 2656.99 & 0.5 \\ 3 & 11 & 4 & 12 & \text { GEN } & 34.99 & 5027.72 & 19.16 & 2585.25 & 0.4 \\ 4 & 12 & 5 & 13 & \text { GEN } & 36.33 & 5027.7 & 19.14 & 2489.86 & 0.5 \\ 5 & 13 & 6 & 14 & \text { GEN } & 38.23 & 5027.69 & 19.12 & 2366.23 & 0.4 \\ 6 & 14 & 7 & 15 & \text { GEN } & 37.49 & 5027.67 & 19.1 & 2413.19 & 0.2 \\ 0 & 8 & 2 & 10 & \text { GEN } & 30.73 & 5027.68 & 19.11 & 7009.87 & 0.4 \\ 1 & 9 & 3 & 11 & \text { GEN } & 33.15 & 5027.7 & 19.12 & 6497.78 & 0.5\end{array}$

Figure 3.12: Example of the data file produced by the ARES datalogger - data file ab1to8rh1 from 08/04/08. 


\subsection{Ice thickness and snow depth measurements}

\subsubsection{Arctic}

As part of their mass balance site (http://seaice.alaska.edu/gi/observatories/barrow_ sealevel, Druckenmiller et al., 2009) the floating ice group at the University of Alaska Fairbanks (UAF) made measurements of the ice thickness and snow level using acoustic measurements. As shown in Figure 3.13, there are three acoustic instruments; above the ice a downwards pointing Campbell SR50 Sonic Ranger to measure the snow thickness, and below the ice two Benthos underwater acoustic altimeter PSA-916, one upward looking to measure ice thickness and one downward looking to measure local sea level. The instruments measure the travel time of an acoustic pulse, and from air and water temperature data calculate the distance to the object it is aimed at. Measurements were made approximately every fifteen minutes and all depths were referenced to the ice/water interface at the time of installation.

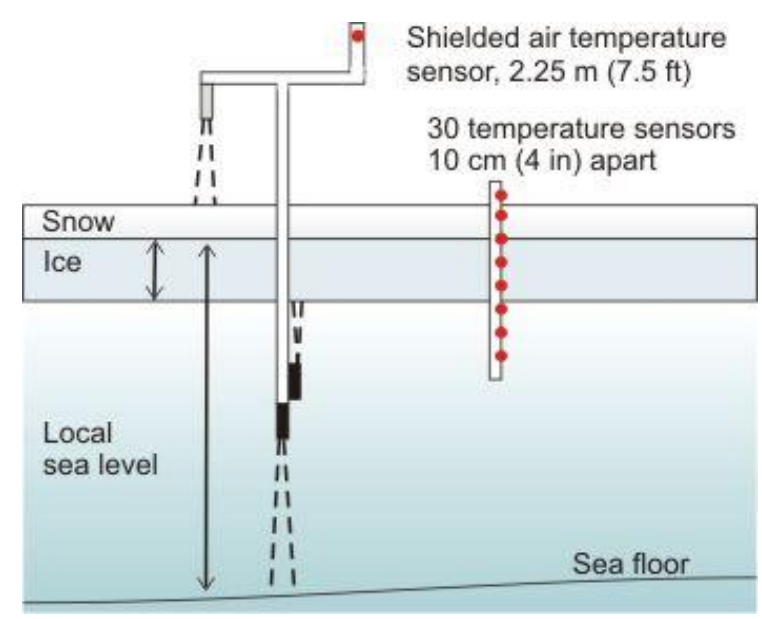

Figure 3.13: Diagram of UAF mass balance site from http://seaice.alaska.edu/gi/observatories/barrow_sealevel.

Figure 3.14 and Figure 3.15 below show the ice thickness and snow depth over the period of the resistivity measurements ( $7^{\text {th }}$ Apr to the $17^{\text {th }}$ Jun, 2008). Note that from early June surface ablation begins to occur. This resulted in incorrect snow depth measurements, which have not been plotted. By the 16-17 June this surface ablation was significant, contributing approximately $0.2 \mathrm{~m}$ towards the decrease of the ice thickness. The decrease in snow depth observed from around the 22 May could mean 
low saline meltwater is introduced to the brine network in the sea ice, flushing some of the brine from the upper inclusions.

\section{Ice thickness over the measurement period}

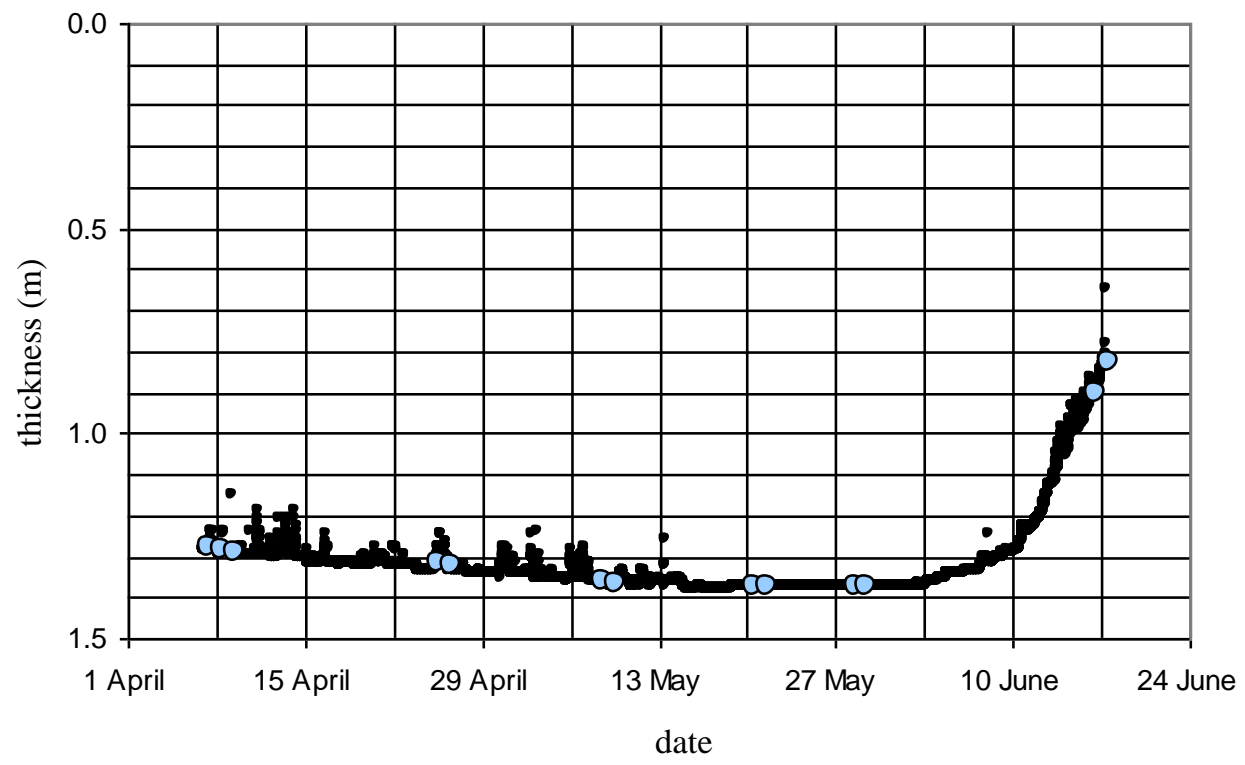

Figure 3.14: Plot of Arctic ice thickness from the 7th Apr to the 17th Jun 2008, measured by an upward looking Benthos underwater acoustic altimeter PSA-916, positioned below the ice at the UAF mass balance site. The ice/air interface is at the top of the plot at $0.0 \mathrm{~m}$ and the blue circles indicate the average on the days on which resistivity measurements were made.

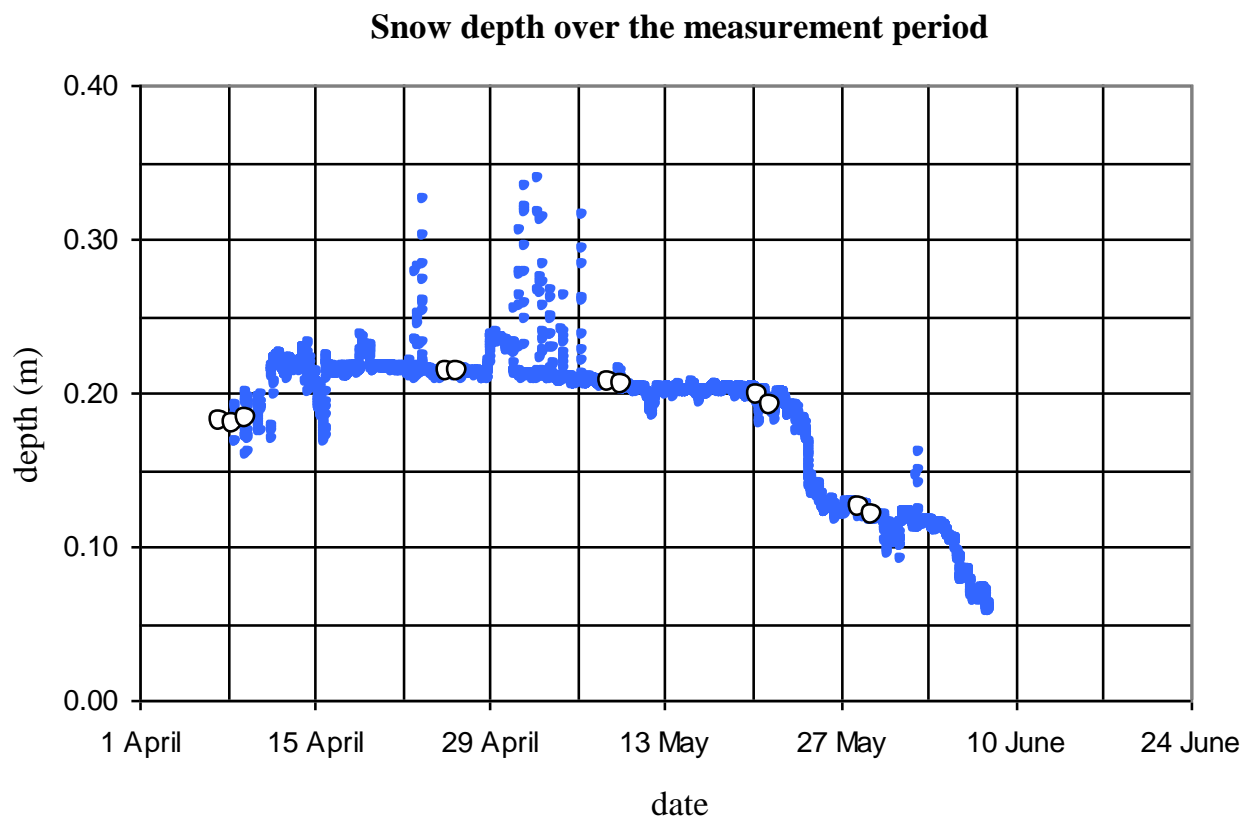

Figure 3.15: Plot of snow depth in the Arctic, from the 7th Apr to the 17th Jun 2008, measured by a downward looking Campbell SR50 Sonic Ranger positioned above the ice at the UAF mass balance site. The snow/ice interface is at the bottom of the plot at $0.00 \mathrm{~m}$ and the white circles indicate the average on the days on which resistivity measurements were made. 


\subsubsection{Antarctica}

The resistivity measurements in the Antarctic were carried out as part of a larger research program, referred to in this thesis as science event K131. As part of this program a mass balance site was set up in the same location as the electrode strings, providing us with measurements of the ice thickness. During the two week measurement period the ice thickness was measured once, on $18^{\text {th }}$ November at two spots with values of $2.4 \mathrm{~m}$ and $2.42 \mathrm{~m}$ giving an average ice thickness of $2.41 \mathrm{~m}$. As the electrode strings are only $1.8 \mathrm{~m}$ long all electrodes were in sea ice during the measurements and (unlike the Arctic data) the ice/water interface is not observed. Measurements of the snow depth on the $18^{\text {th }}$ November were $0.13 \mathrm{~m}$ and $0.19 \mathrm{~m}$ respectively, giving an average snow depth of $0.16 \mathrm{~m}$. 


\subsection{Temperature measurements}

\subsubsection{Arctic}

Over the period of the resistivity measurements, the temperature in the ice was recorded using thermistor strings. These strings were frozen into the sea ice near the site of the electrode strings, as part of the mass balance site maintained by the University of Alaska Fairbanks (see Figure 3.13). The thermistors were positioned every $10 \mathrm{~cm}$, starting at the surface of the ice and going to a depth of $240 \mathrm{~cm}$. Temperature measurements were made approximately every fifteen minutes. Figure 3.17, at end of this section, shows the temperature data obtained from these thermistor strings over the period $7^{\text {th }}$ of April to the $17^{\text {th }}$ of June, 2008, as well as measurements of the air and water temperatures.

The average temperature at each of the thermistors was obtained over the period of each of the resistivity measurement sets. This allowed a temperature profile with depth to be created for each of the measurement sets, see Figure 3.16 below.

Temperatures with depth on days that resistivity measurements occurred

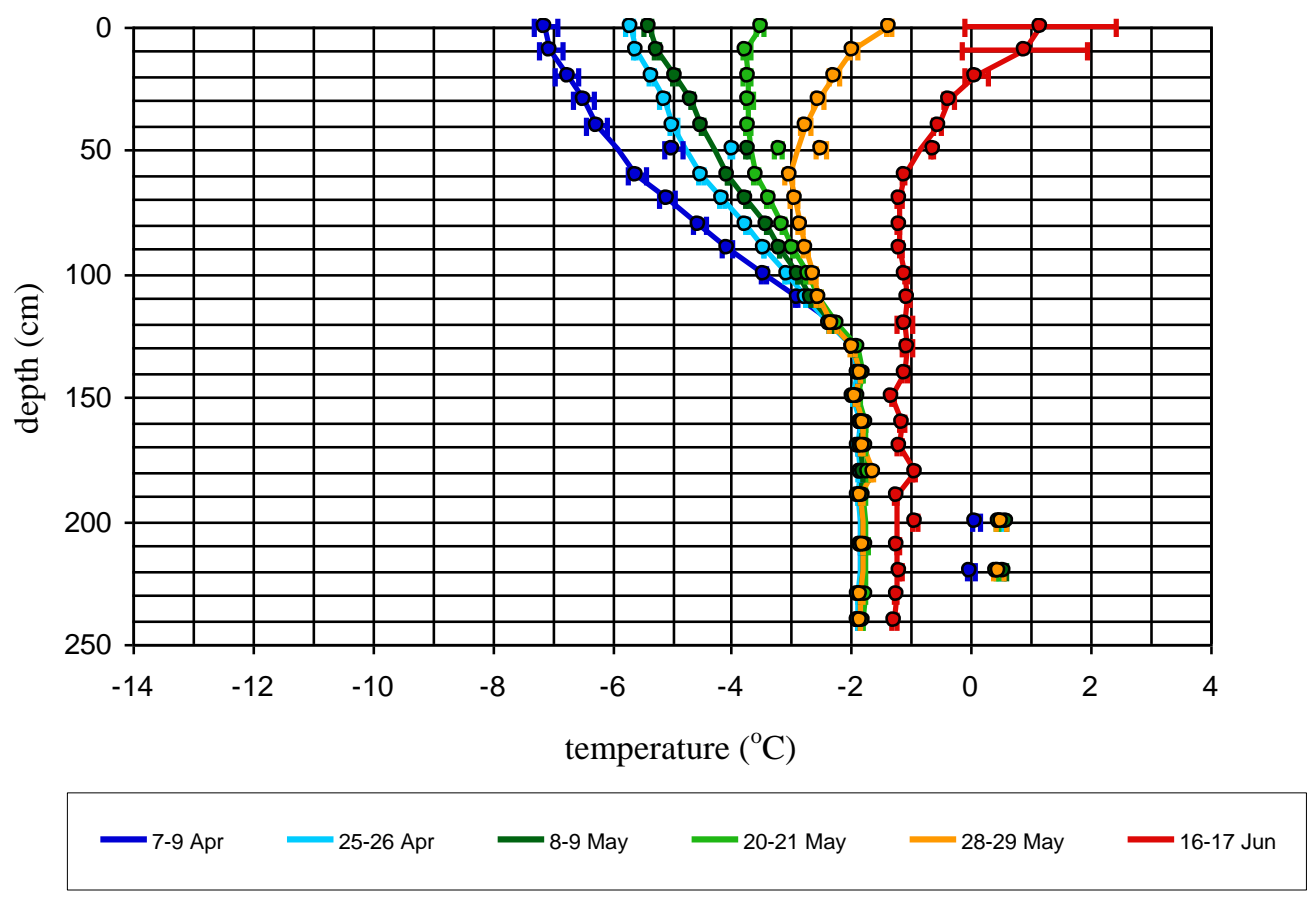

Figure 3.16: Plot of averaged temperature with depth over the period of each measurement set of the 2008 Arctic measurements. The surface of the ice is at the top of the plot and the error bars represent the standard deviation of the averaged data. 
Three of the thermistors did not work correctly, which can be observed in Figure 3.17a and Figure 3.16, with thermistors at depths of $50 \mathrm{~cm}, 200 \mathrm{~cm}$ and $220 \mathrm{~cm}$ giving anomalous readings. As the deeper thermistors are within the seawater, well below the ice, (see ice thickness plot - Figure 3.14) they may be ignored, as they do not affect our study of the ice. However at a depth of $50 \mathrm{~cm}$ the temperature plot has been smoothed, by averaging the temperatures at depths of $40 \mathrm{~cm}$ and $60 \mathrm{~cm}$.

As would be expected over the measurement period (the $7^{\text {th }}$ April to the $17^{\text {th }}$ June) Figure 3.17a and Figure 3.16 show the temperature of the ice increases, with surface temperatures of about $-7^{\circ} \mathrm{C}$ on the 7-9 Apr increasing to $\sim 0^{\circ} \mathrm{C}$ on the $16-17$ Jun. For the last resistivity measurement set, carried out on the 16-17 June, the upper few thermistors record positive temperatures. This indicates surface ablation has occurred and the upper thermistors are no longer in the ice, which is confirmed by observations at the electrode strings during this final measurement set.

Typically the temperature profile of an ice cover decreases from air temperatures at the surface of the ice to the freezing temperature of seawater at the base. As the air temperature rises the increase is propagated downward through the ice initially warming the upper regions of the cover. For data from the 7-9 Apr, 25-26 Apr and 8-9 May the temperature of the ice is lowest at the surface and increases with depth (due to insulation from the ice above) till it reaches the relatively fixed temperature, of $\sim-2^{\circ} \mathrm{C}$, of the seawater below. For data from the 20-21 May and 28-29 May the air temperature has increased sufficiently (Figure $3.17 \mathrm{~b}$ shows a jump in air temperature from $-10^{\circ} \mathrm{C}-$ $-7^{\circ} \mathrm{C}$ on 8-9 May to $-4^{\circ} \mathrm{C}--1^{\circ} \mathrm{C}$ for the later measurements) that the upper regions of the ice are warmer than the temperatures measured further down. Thus we see a C-shaped curve with the temperature decreasing with depth then increasing until reaching the seawater value. On the 16-17 Jun the air temperature is above $0^{\circ} \mathrm{C}$ and slightly higher temperatures at the surface of the ice decrease to a relatively constant value of $\sim-1^{\circ} \mathrm{C}$ from around $0.5 \mathrm{~m}$ downwards. 
(a) Temperatures in the ice over the measurement period

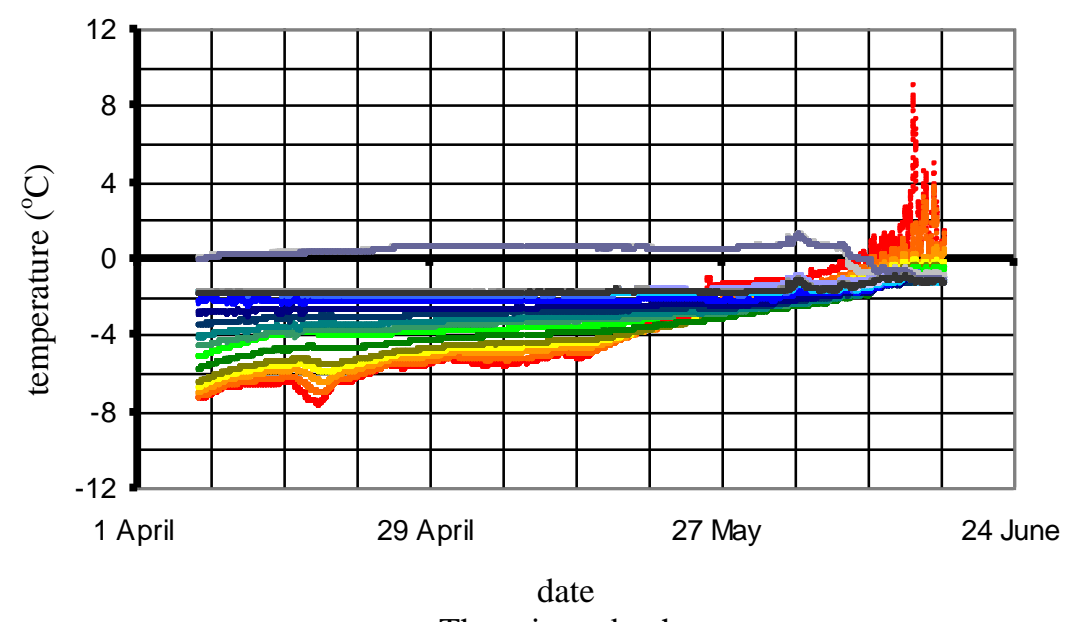

Thermistor depths

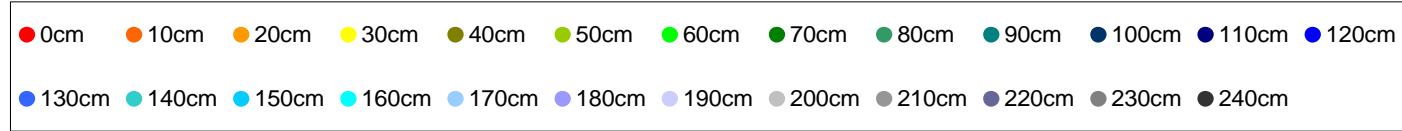

(b) Air temperature over the measurement period

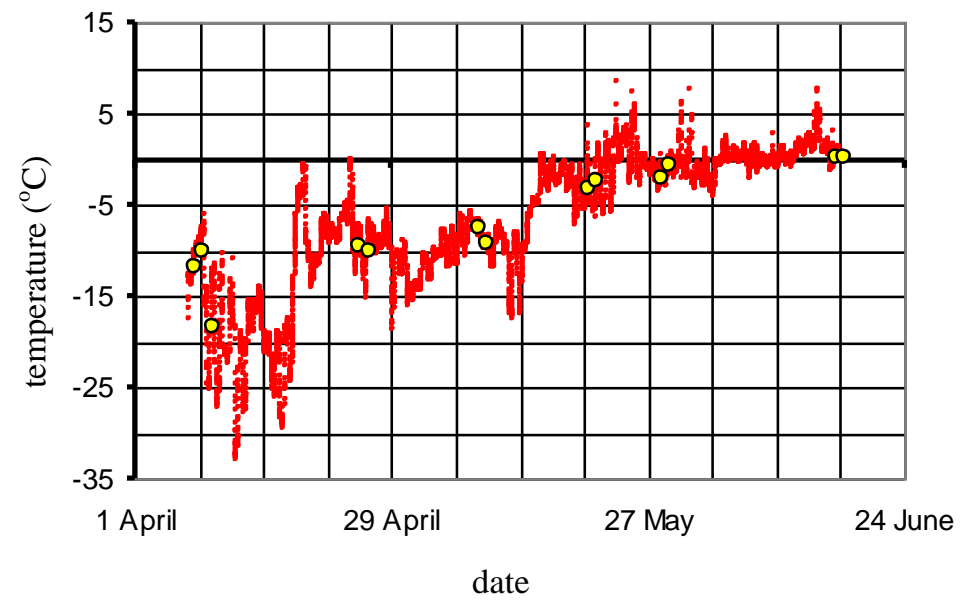

(c) Water temperature over the measurement period

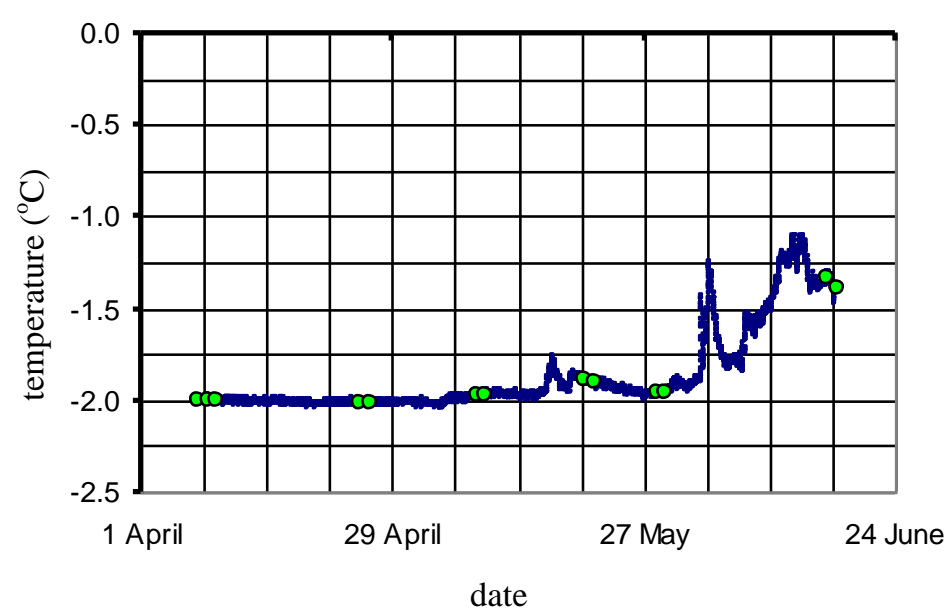

Figure 3.17: Plot of temperatures over the Arctic measurement period 7th Apr to 17th Jun 2008. (a) temperature measured by thermistors buried in the ice at intervals of $10 \mathrm{cms}$. (b) air temperature measured by shielded sensor $2.25 \mathrm{~m}$ above ice surface - yellow circles indicate dates of the resistivity measurement sets. (c) water temperatures - green circles show when resistivity measurements were made. All measurements were made at the UAF mass balance site. 


\subsubsection{Antarctica}

Temperatures were measured, often several times a day, near the resistivity measurement site by other members of the K131 group. Figure 3.18 below displays averaged temperature with depth on the days resistivity measurements were made.

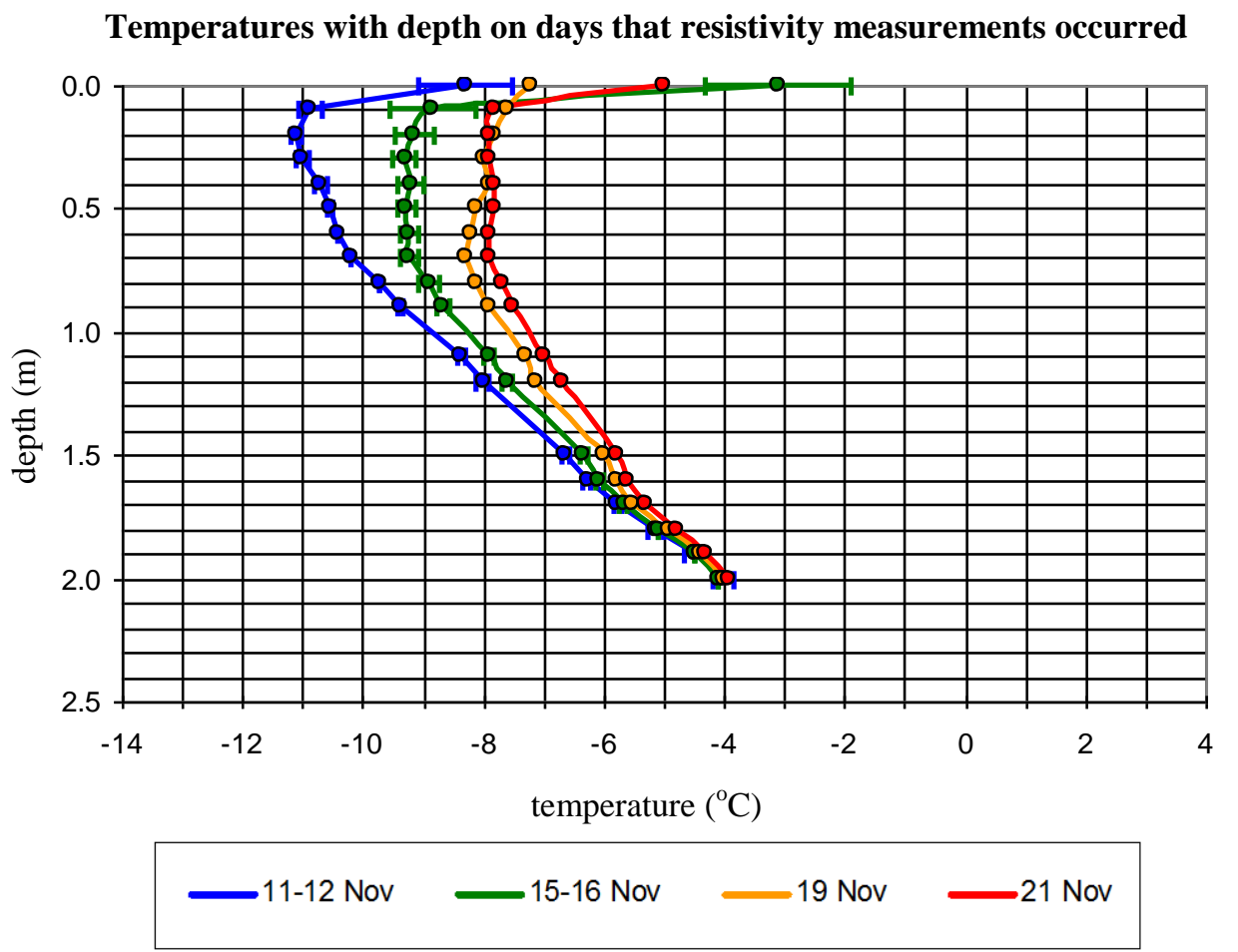

Figure 3.18: Plot of temperature with depth over the period of each measurement set of the 2009 Antarctic data. The surface of the ice is at the top of the plot and error bars show the standard deviation of the averaged values.

From Figure 3.18 it can be seen that the surface temperature of the sea ice is relatively high and decreases to its coldest temperature at a depth of $0.1 \mathrm{~m}$ to $0.3 \mathrm{~m}$. From this depth to further in the ice (the ice/water interface is not reached) the temperature increases gradually. Similar temperature profiles with depth are observed in the 2008 Arctic data (Figure 3.16), in particular from the $20^{\text {th }}$ of May onwards. However, the temperatures measured in the Antarctic reach lower values than those observed during the Arctic measurements. Additionally the range of temperatures between profiles is smaller during the shorter period of Antarctic measurements. 


\subsection{Salinity measurements}

\subsubsection{Arctic}

Curves of the salinity through the ice were obtained by UAF through the standard process of electrolytical conductivity measurements on melted ice cores. Cores were taken on 7 Apr, 29 Apr, 26 May and 16 Jun in and around the mass balance and resistivity measurement site. Figure 3.19 below displays the salinity with depth. The salinity profile of the ice during the resistivity measurements are taken as that recorded on the nearest date to resistivity measurements, i.e. 7 Apr (7-9 Apr), 29 Apr (25-26 April, 8-9 May), 26 May (20-21 May, 28-29 May) and 16 Jun (16-17 Jun).

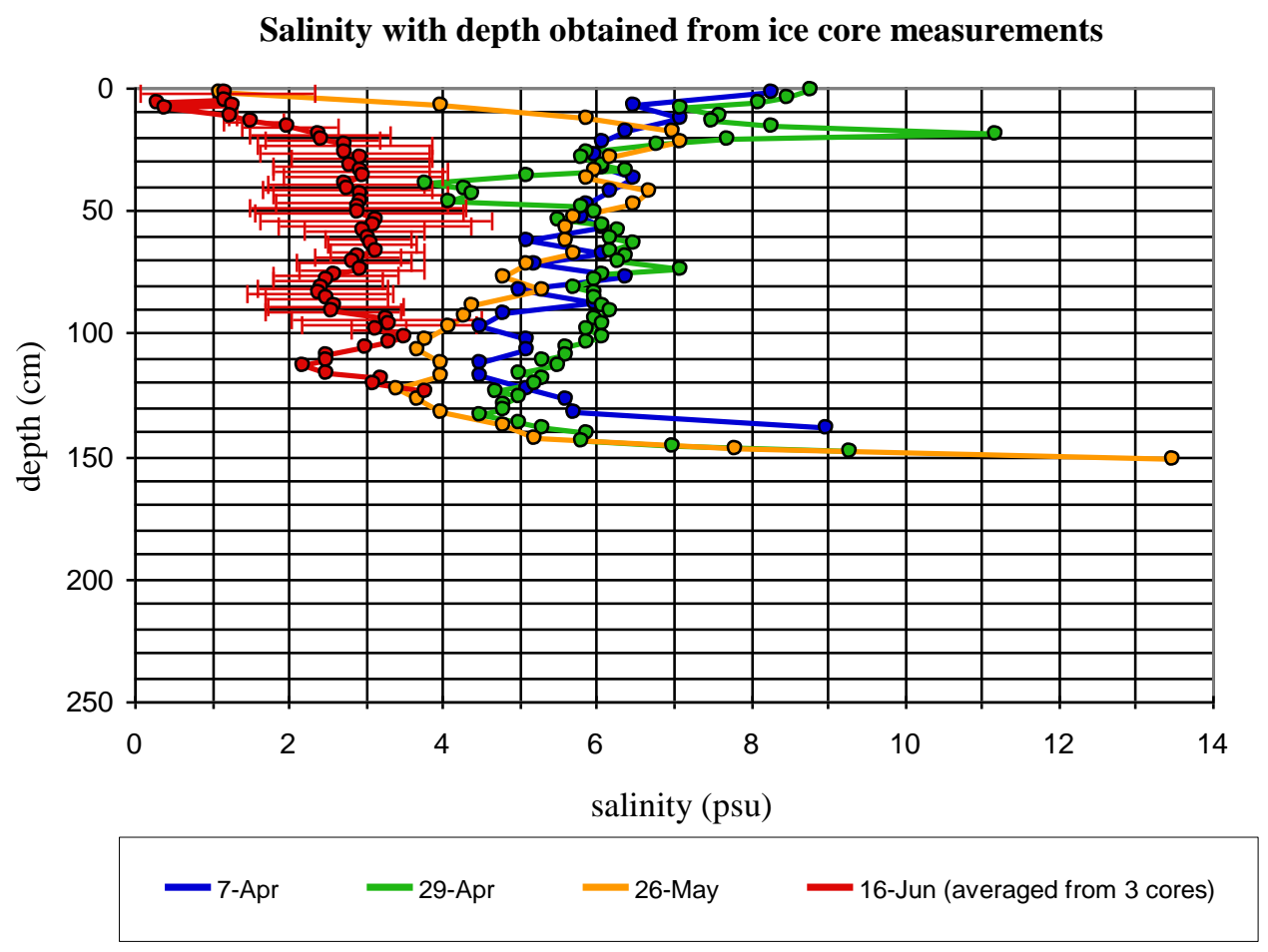

Figure 3.19: Plot of salinity vs. depth, salinities obtained from ice cores taken by UAF during 2008 Arctic measurements. Curve for the 16 Jun is the average of three cores and the error bars represent the standard deviation of the average values.

The salinity curves in Figure 3.19 show a general decrease in the salinity of the ice as the temperature warms. This is as expected as with increasing temperatures the brine inclusions become larger and interconnected and melting ice dilutes the brine. The salinity measurements from 7 Apr and 29 Apr produce C-shaped plots with higher surface salinities which decrease with depth then increase again with the highest 
salinities measured at the bottom of the ice. The salinity measurements from 26 May and 16 Jun show a reduction in surface salinities, so that the surface gives the lowest salinities which then begin to increase with depth before once again producing a $\mathrm{C}$-shaped curve. The $\mathrm{C}$-shape of the 16 Jun plot is small and relatively shallow.

These observed trends follow the typical salinity profile, with depth, of young and firstyear Arctic ice. This follows a characteristic C-shape (Eicken, 2003), with higher salinities at the base and top of the ice cover. Rapid freezing rates result in maximum trapping of brine, while low freezing rates permit seepage of brine before solidification is complete (Eicken, 2003). The high salinity in the upper layers of the ice is probably due to the fast freezing of randomly orientated frazil ice trapping a significant volume of brine. The salinity then decreases with depth due to a slowing in the growth rate, gravity drainage, brine expulsion and migration of brine pockets in temperature gradients. When the newly formed ice at the bottom of the cover is reached salinity becomes high again, as this region has had less time to lose any brine element.

The profiles also display the expected reduction in surface salinity (Eicken, 2003), as the temperatures increase and the ice becomes more porous with age its salinity decreases. The salinity of the surface and base of the ice generally decreases faster then the middle of the cover. The decreasing salinity is due to the production of low salinity meltwater at the surface and base of the ice, which is capable of displacing higher salinity brine from within the ice, and brine drainage through the increasingly connected brine inclusions of the porous ice.

Comparing the 2008 profiles from Figure 3.19 with the evolution of the typical Arctic sea ice salinity profile displayed in Eicken (2003) (Figure 3.20 below) the April data are similar to the March profile, the May data to the June profile and the June data seem to follow the August profile.

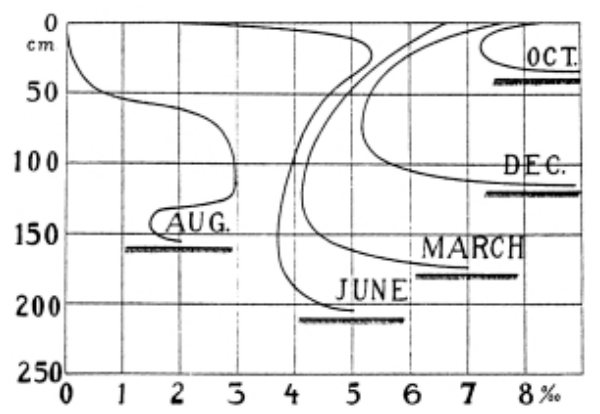

Figure 3.20: Evolution of Arctic sea ice salinity profiles (from Malmgren, 1927). 


\subsubsection{Antarctica}

Two profiles of salinity with depth through the Antarctic ice were obtained during the resistivity measurement period, as part of K131 research. These profiles were combined, and measurements made at the same depth averaged, to produce the salinity with depth profile plotted below in Figure 3.21.

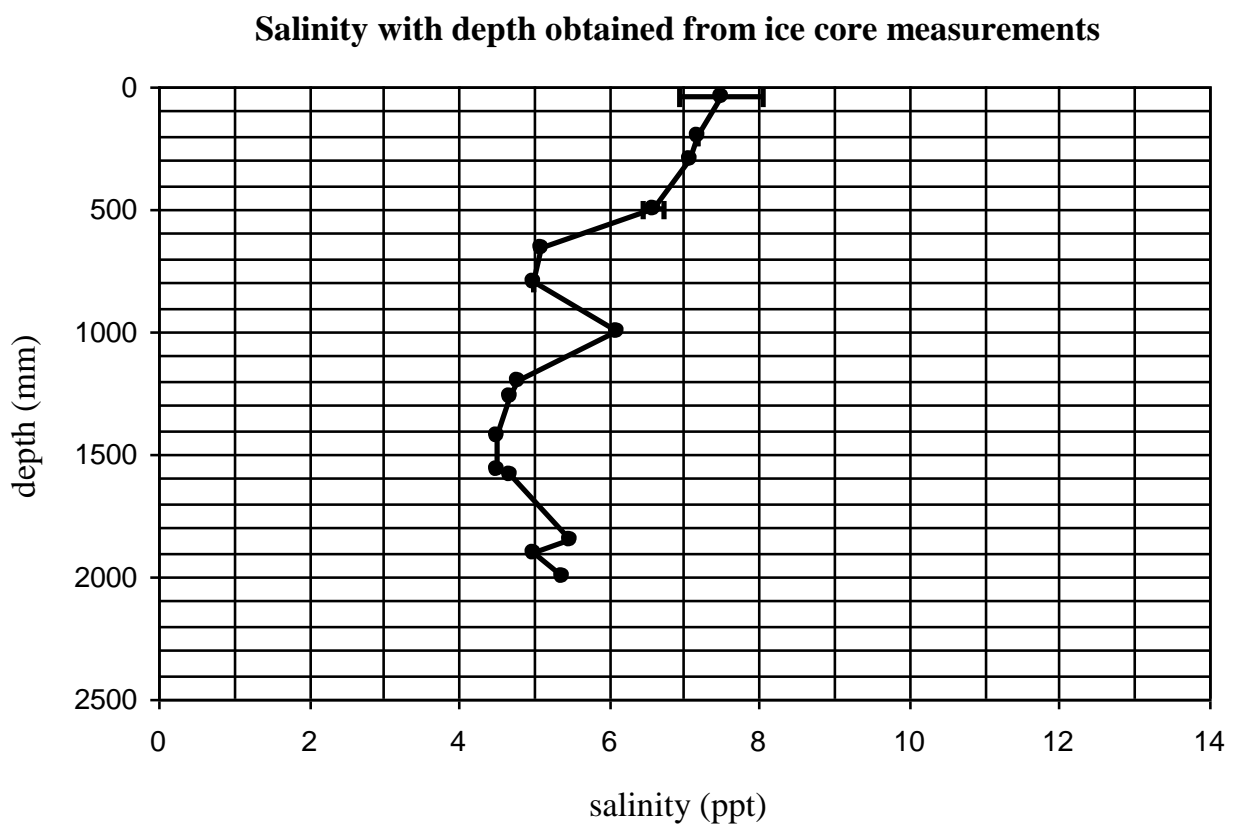

Figure 3.21: Plot of salinity vs. depth, salinities obtained from ice cores taken by K131 members during 2009 Antarctic measurements. Error bars show the standard deviation of averaged values

The salinity data in Figure 3.21 shows high surface salinities which decrease with depth then increase slightly towards the bottom of the ice, with values similar to those measured on 7 April and 29 April 2008. This general trend is disrupted by a peak in salinity at $1000 \mathrm{~mm}$ or $1 \mathrm{~m}$.

These observed trends follow the "S-type" salinity profiles observed by Eicken (1992) during ice core measurements made in the Weddell Sea, Antarctica. The S-type profiles are similar to the C-shape profiles typical of young and first-year Arctic ice. However, while the salinities are high at the surface the S-type profile shows stagnating or minimum values at the base. The S-type profiles were the most common salinity profile of Antarctic sea ice observed by Eicken (1992). 


\subsection{Brine volume fraction calculations}

\subsubsection{Arctic}

The brine volume fraction $\left(V_{b} / V\right)$ of the ice can be calculated according to Cox and Weeks (1983):

$$
V_{b} / V=\rho S_{s i} / F_{l}(T)
$$

where - $\rho$ is the bulk density of the ice (approximated as $0.91 \mathrm{gcm}^{-3}$, the average value stated by Timco and Frederking (1996))

- $S_{s i}$ is the bulk salinity of the sea ice (obtained from the UAF core data)

$-F_{l}(T)=-0.041221-18.407 T+0.58402 T^{2}+0.21454 T^{3} \quad\left(\right.$ for $\left.-2^{\circ} \mathrm{C}<T \leq 0^{\circ} \mathrm{C}\right)$ and

$$
F_{l}(T)=-4.732-22.45 T-0.6397 T^{2}-0.0174 T^{3} \quad\left(\text { for }-22.9^{\circ} \mathrm{C} \leq T \leq-2^{\circ} \mathrm{C}\right)
$$

(as given in Eicken (2003))

The results for these calculations are displayed below in Figure 3.22.

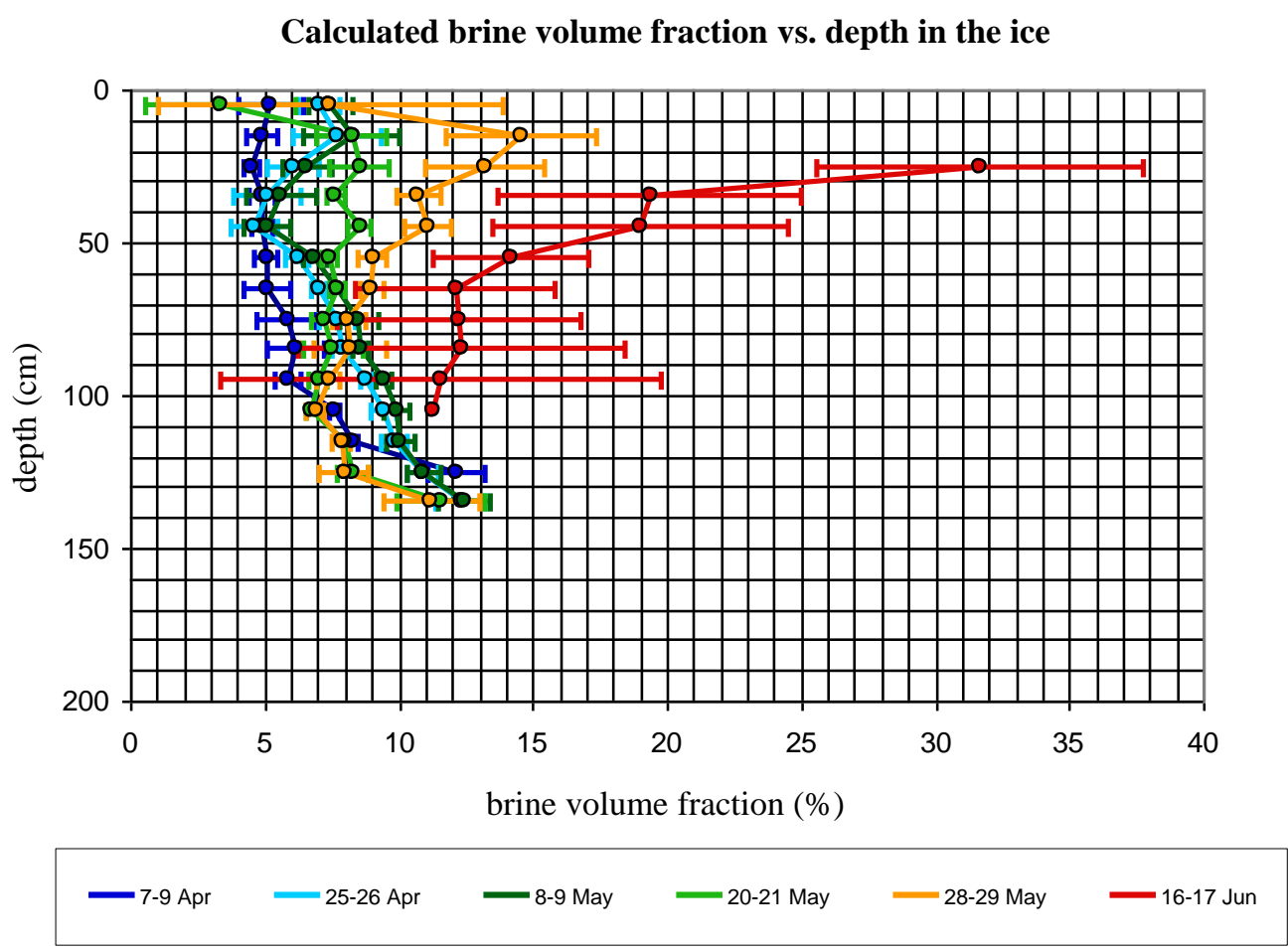

Figure 3.22: Plot of calculated brine volume fraction vs. depth for 2008 Arctic data. The error bars give an indication of the error in the calculation, arising from the standard deviations of the averaged temperatures and salinities. 
The general trend of the profiles show higher brine volume fractions at the base of the cover, where the ice is most recently formed. As the brine volume fraction is a function of temperature its values increase as the temperatures increase over the measurement period. This increasing trend would be expected for a growing brine inclusion network

The calculated brine volume fraction profile for 7-9 Apr shows a slight decrease in brine volume fraction in the upper regions followed by a slow increase with depth to about $6 \%$ at $1 \mathrm{~m}$, brine volume fraction then increases significantly reaching $\sim 12 \%$ at $1.25 \mathrm{~m}$. For 25-26 Apr and 8-9 May the brine volume fraction sees a brief increase from surface values before decreasing to about $5 \%$ at $0.45 \mathrm{~m}$ then increasing again to reach $\sim 12 \%$ at $1.35 \mathrm{~m}$. The 20-21 May and 28-29 May curves have a much more pronounced increase from surface brine volume fractions, than observed on the 25-26 Apr, this increase is then followed by a C-shaped curve. For the 20-21 May the brine volume fraction increases from $\sim 3 \%$ at the surface to $\sim 9 \%$ at $0.25 \mathrm{~m}$, from here the $\mathrm{C}$-shape begins and the curve decreases to $\sim 7 \%$ at $1 \mathrm{~m}$ then increases to $12 \%$ at the base of the ice. The 28 29 May on the other hand starts off with a surface brine volume fraction of $8 \%$ increasing to $15 \%$ at $0.15 \mathrm{~m}$, from here the curve follows a $\mathrm{C}$-shape and decreases to $\sim 7 \%$ at $1 \mathrm{~m}$ then increases to $11 \%$ at the base of the ice. The appearance of low values of brine volume fraction at the surface of the ice coincides with high temperatures and low salinity measurements. Increasing temperature should mean an increase in the size and connectivity of the brine inclusions leading to an increase in brine volume fraction. However, with increasing temperature there is also a decrease in salinity as meltwater enters the brine network. If this decrease in significant enough it can lead to a decrease in the brine volume fraction even though the connectivity of the brine network is high. By the 16-17 Jun large values of $\sim 32 \%$ at the surface of the ice decrease to $11 \%$ at the base of the ice. This indicates the large effect of the relatively high temperatures on the structure of the ice. Though the low salinity profile on this date suggests that while greatly enlarged the brine structure is diluted by meltwater. As mentioned earlier, the temperatures on the 16-17 Jun at the first few thermistors exceed $0^{\circ} \mathrm{C}$, due to being exposed by surface ablation. Thus the surface of the ice on the 16-17 June is considered to be at $20 \mathrm{~cm}$ (rather than $0 \mathrm{~cm}$ ) on this plot. 


\subsubsection{Antarctica}

As with the Arctic data above, the brine volume fraction $\left(V_{b} / V\right)$ of the Antarctic ice is calculated and displayed below in Figure 3.23. For the Antarctic data only one salinity profile is obtained from measurements. As temperatures were measured on the salinity cores this data is used along with the one salinity profile to calculate the brine volume fraction using the expression in section 3.7.1. Thus there is only one brine volume fraction profile for the Antarctic ice, not one for each measurement period. As there is little change in the temperature over the measurement period of the Antarctic data one brine volume fraction profile should suffice.

Calculated brine volume fraction vs. depth in the ice

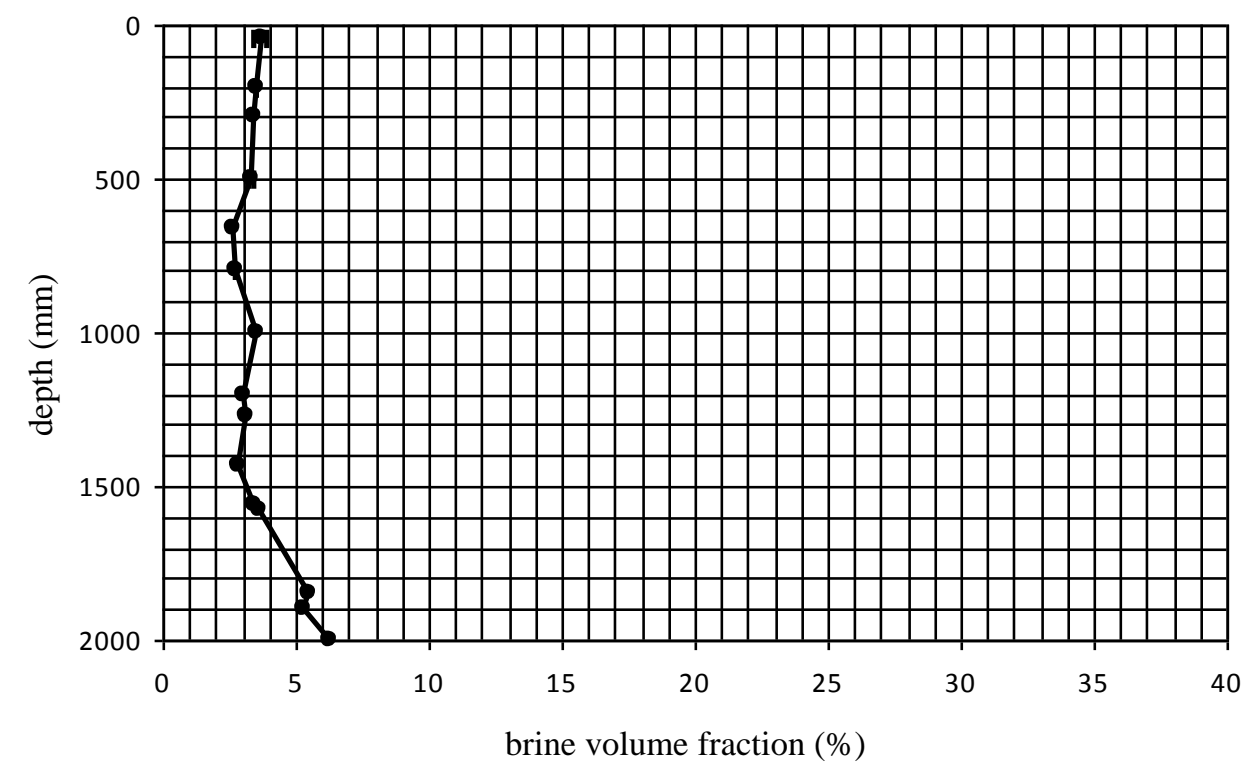

Figure 3.23: Plot of calculated brine volume fraction vs. depth for 2009 Antarctic data. The error bars give an indication of the error in the calculation, arising from the standard deviations of the averaged temperatures and salinities.

The observed profile follows a curved plot with low surface brine volume fractions increasing with depth. The plot is comparable to the early April measurements from our 2008 Arctic measurements (Figure 3.22). However, the calculated brine volume fractions are slightly lower than those recorded in the Arctic and the increase with depth is not as significant. 


\subsection{Brine salinity calculations}

\subsubsection{Arctic}

Values of the salinity of the brine can be calculated from the temperature of the ice using the following expressions, from Lepparanta and Manninen (1988):

$$
\begin{array}{lr}
S_{b}=0.356 T^{2}-18.95 T-0.1201 & \left(\text { for }-2^{\circ} \mathrm{C} \leq T \leq 0^{\circ} \mathrm{C}\right) \\
S_{b}=1000(1-(54.11 / T))^{-1} & \left(\text { for }-8.2^{\circ} \mathrm{C} \leq T<-2^{\circ} \mathrm{C}\right) \\
S_{b}=1000(1+1 /(0.082-0.00848 T))^{-1} & \left(\text { for }-22.9^{\circ} \mathrm{C} \leq T<-8.2^{\circ} \mathrm{C}\right)
\end{array}
$$

Figure 3.24 below shows the calculated profiles of the brine salinity against depth for each of the measurement sets.

Calculated brine salinity vs. depth in the ice

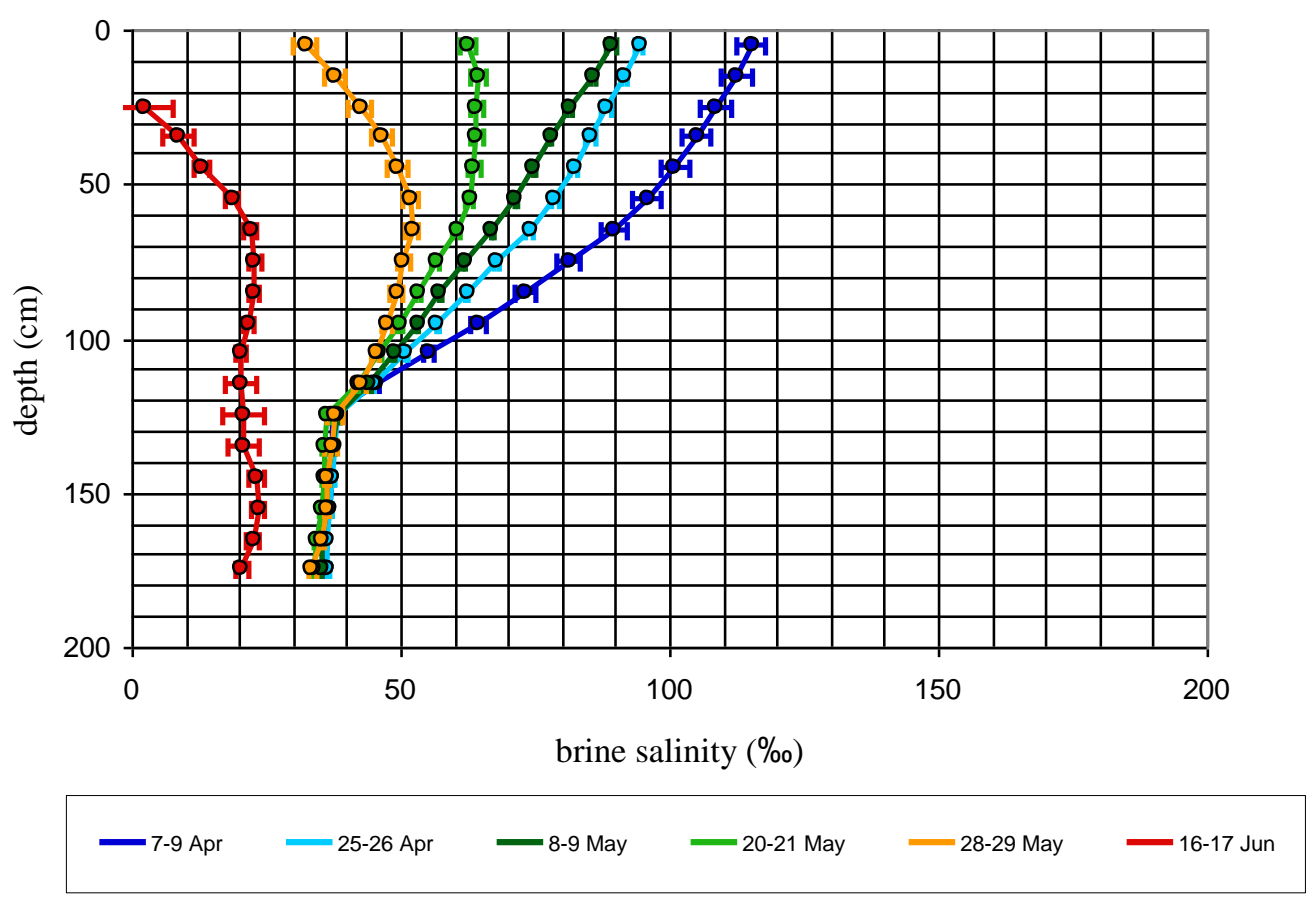

Figure 3.24: Plot of calculated brine salinity in parts per thousand vs. depth in centimetres for 2008 Arctic data. The error bars give an indication of the error in the calculation, arising from the standard deviations of the averaged temperatures.

As expected, see section 1.2.2, Figure 3.24 shows that the calculated brine salinity decreases as the temperatures increase over the measurement period. For the 7-9 Apr, 25-26 Apr and 8-9 May the brine salinity profiles have their highest values at the surface of the ice and decrease with depth in the ice. There is an increase in this rate of 
decrease at approximately $55 \mathrm{~cm}$ then at around $125 \mathrm{~cm}$ the brine salinity hits $\sim 35 \%$ othe usual salinity of seawater. The decrease in brine salinity with depth is due to the increasing temperatures deeper in the ice. From the 20-21 May and 28-29 May a region of low surface brine salinities is observed, with brine salinities now initially increasing with depth before decreasing towards the $35 \%$ value of seawater. The brine salinities calculated for the 16-17 Jun data have this same low surface region followed by an increase then decrease with depth. However, the calculated brine salinities for this data set are significantly lower than those obtained for the other data sets, never reaching above $25 \%$. This corresponds to the significant increase in temperatures between 28-29 May and 16-17 Jun. As mentioned earlier the upper values of the 16-17 Jun are missing due to high temperatures $\left(>0^{\circ} \mathrm{C}\right)$ recorded here as the upper thermistors are exposed due to surface ablation of the ice cover.

\subsubsection{Antarctica}

For the Antarctic sea ice brine salinity is calculated from the temperature of the ice using the expressions given in section 3.8.1 above. The results of these calculations are shown in Figure 3.25 below, which displays the profiles of the brine salinity against depth for each of the measurement sets.

Calculated brine salinity vs. depth in the ice

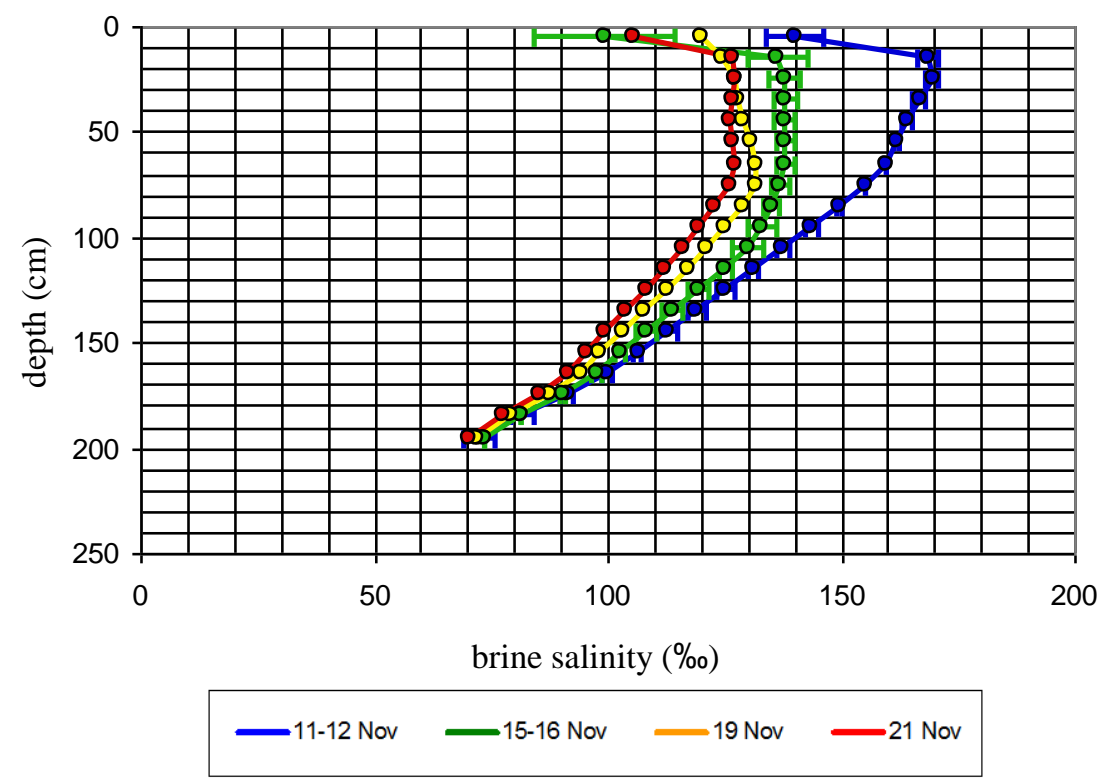

Figure 3.25: Plot of calculated brine salinity in parts per thousand vs. depth in centimetres for 2009 Antarctic data. The error bars give an indication of the error in the calculation, arising from the standard deviations of the averaged temperatures. 
Figure 3.25 shows a slight decrease in calculated brine salinity over the measurement period, coinciding with increasing temperature. The plots indicate low brine salinity in the relatively warm ice at the surface that increases with depth, then decreases towards the bottom of the ice. The shapes of the curves are similar to those obtained from measurements made in late May, 2008 in the Arctic. However, as the ice/sea interface is not reached in the Antarctic no decrease to $\sim 35 \%$ is observed. Furthermore due to the colder temperatures recorded in Antarctica the brine salinity is higher than that observed in the Arctic. 


\subsection{Brine resistivity calculations}

\subsubsection{Arctic}

Values for the brine conductivity can be calculated from the brine salinity values above and the temperature of the ice using the following equations from Stogryn (1971)

$$
\begin{gathered}
\sigma_{N a C I}(T, N)=\sigma_{N a C l}(25, N)\left[1.000-1.962 \times 10^{-2} \Delta+8.08 \times 10^{-5} \Delta^{2}-\Delta N\left\{3.020 \times 10^{-5}+\right.\right. \\
\left.\left.3.922 \times 10^{-5} \Delta+N\left(1.721 \times 10^{-5}-6.584 \times 10^{-6} \Delta\right)\right\}\right]
\end{gathered}
$$

where $\Delta=25-T$

$$
\begin{aligned}
& \sigma_{N a C l}(25, N)=N\left[10.394-2.3776 N+0.68258 N^{2}-0.13538 N^{3}+1.0086 \times 10^{-2} N^{4}\right] \\
& N=S_{b}\left[1.707 \times 10^{-2}+1.205 \times 10^{-5} S_{b}+4.058 \times 10^{-9} S_{b}^{2}\right]
\end{aligned}
$$

$S_{b}$ is the salinity of the brine

The brine resistivity is then simply the inverse of the calculated conductivity. The profiles of the calculated brine resistivities with depth are displayed in Figure 3.26 below.

Calculated brine resistivity vs. depth in the ice

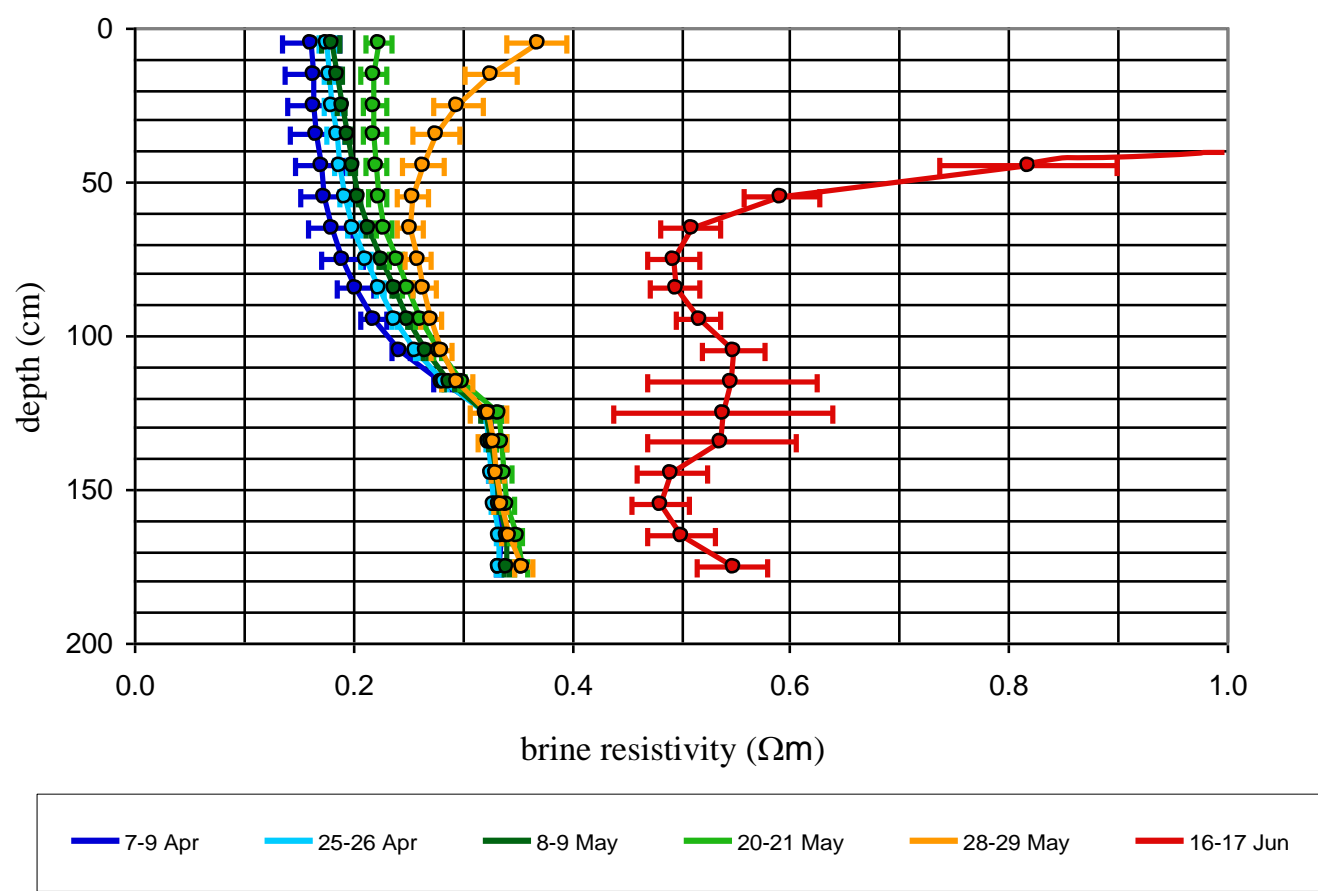

Figure 3.26: Plot of calculated brine resistivity in ohm meters vs. depth in centimetres for 2008 Arctic data. The error bars give an indication of the error in the calculation, arising from the standard deviations of the averaged temperatures and the errors in the calculation of the brine salinity. 
Recall, that above $\sim-8^{\circ} \mathrm{C}$, as the temperature decreases the brine salinity and hence conductivity increases (see section 1.3). Thus as resistivity is the inverse of conductivity one would expect the plots of brine resistivity to increase with increasing temperature and to be the mirror image of the brine salinity plots, and this is generally what is observed in Figure 3.26. The calculated brine resistivities increase over the measurement period as the ice warms and the brine becomes less saline. For 7-9 Apr, 25-26 Apr, 8-9 May, and 20-21 May the brine resistivity profile starts off with low surface values that increase with depth (and increasing temperature within the ice), following a curved plot until reaching a value of $\sim 0.35 \Omega \mathrm{m}-$ which is consistent with measurements of the resistivity of seawater (Thyssen et al., 1974; Buckley et al., 1986; Haas et al., 1997; Ingham et al., 2008). The move to high surface brine resistivities does not seem to occur until 28-29 May. This is slightly later than the observed decrease in surface brine salinity and increase in surface temperatures. On 28-29 May the calculated brine resistivities decrease with depth before increasing to the seawater value, forming a C-shaped curve. The brine resistivities calculated for 16-17 Jun are significantly higher than those observed in the other data sets, with high surface values (that extend off the plot, reaching $4 \Omega \mathrm{m}$ ) that decrease with depth until reaching a relatively constant value. Once again the upper values of the calculated brine resistivity for 16-17 Jun are missing due to surface ablation.

\subsubsection{Antarctica}

As for the Arctic ice above, values for the brine resistivity can then be calculated from the brine salinity values and the temperature of the Antarctic ice. The profiles of the calculated brine resistivities with depth are displayed in Figure 3.27 below.

In the Antarctic data the brine resistivity is effectively the same over all four measurements with values of the order of $0.15 \Omega \mathrm{m}$ to $0.2 \Omega \mathrm{m}$. The shape of the curves is a shallow $\mathrm{C}$ with higher surface resistivity that decreases slightly, then increases with depth. As expected this pattern is more or less the inverse of that observed in the brine salinity calculations, and shows higher brine resistivities where higher temperatures are observed. The shape of the curves follow a similar trend to those obtained from 2008 Arctic measurements, though the $\mathrm{C}$-shape is more shallow and the values lower (corresponding to the higher brine salinity calculated for the Antarctic data). 
Furthermore, as the ice/sea interface is not reached in the Antarctic no constant value is reached at depth.

\section{Calculated brine resistivity vs. depth in the ice}

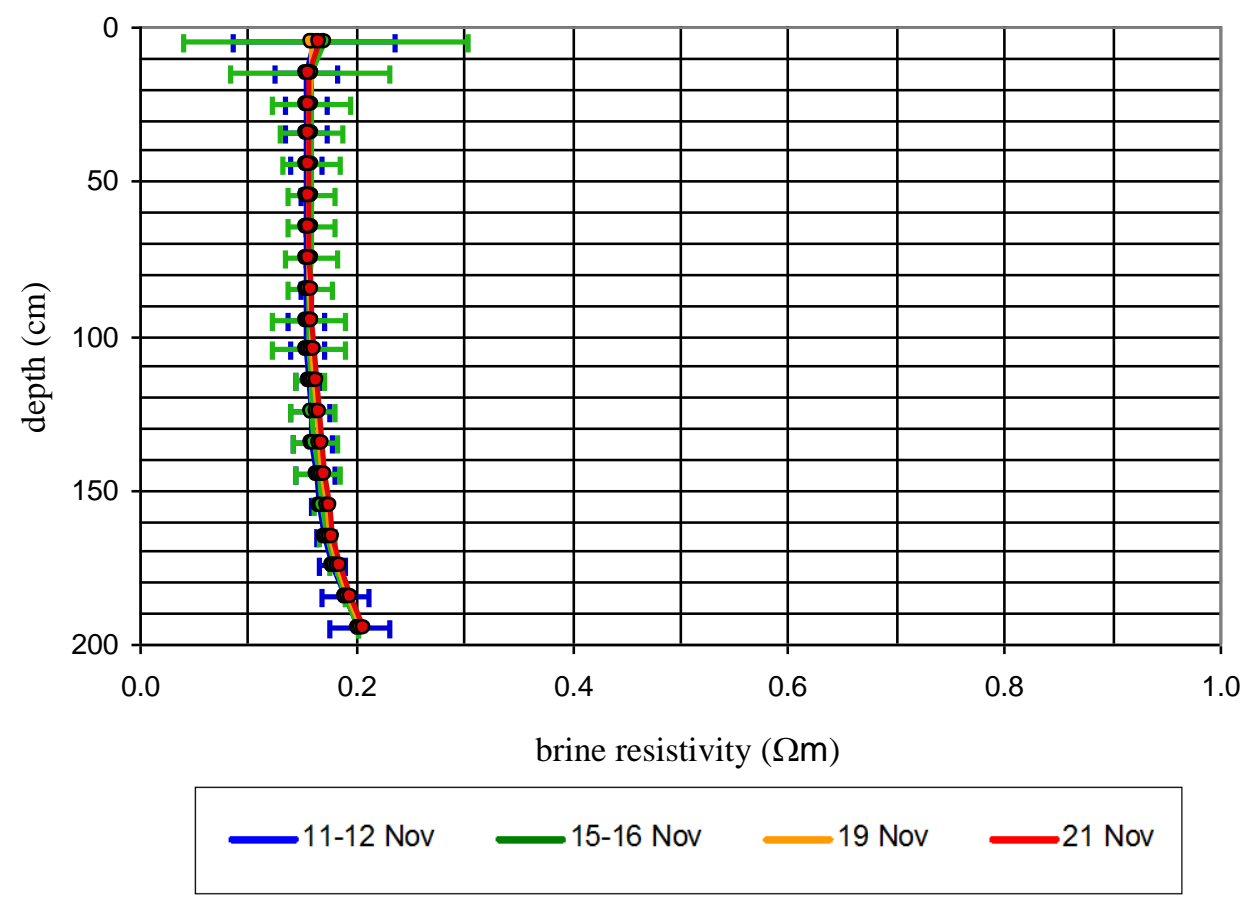

Figure 3.27: Plot of calculated brine resistivity in ohm meters vs. depth in centimetres for 2009 Antarctic data. The error bars give an indication of the error in the calculation, arising from the standard deviations of the averaged temperatures and the errors in the calculation of the brine salinity. 


\subsection{Summary}

Measurements, from which values of the horizontal and geometric mean resistivity structure of first-year sea ice are obtained, were carried out for both a three month period in the Arctic (April - June 2008) and a two week period in the Antarctic (November 2009). Measurements were made between combinations of four electrode strings positioned in boreholes in the ice. These strings were connected to programmable 'smart' electrodes and an ARES or ABEM datalogger. Different programmes were used to select the required electrode combinations for mostly automated horizontal and geometric mean resistivity measurements.

Additional to the resistivity measurements other parties (UAF and Science event K131) recorded other important properties of the sea ice, such as ice thickness, temperature and salinity. Using these measurements it is possible to calculate values for the brine volume fraction, brine salinity and brine resistivity. Knowledge of these properties of the sea ice helps with interpretation of the resistivity measurements.

From the plots in this chapter, displaying the properties of the Arctic ice in 2008, it can be seen that as the temperatures increase over the measurement period the following changes occur. In general (i) ice salinity decreases; (ii) the calculated brine volume fraction increases; (iii) calculated values of brine salinity decrease and (iv) computed brine resistivity values increase.

These observations may be explained conceptually as increasing temperatures corresponds to melting of the snow and ice. This generally leads to increased size and connectivity of the brine pockets and the introduction of less saline melt water into this network. The increase in the size and connectivity of the brine pockets would explain the observed increase in brine volume fraction while allowing a decrease in the ice and brine salinity and thus an increase in the brine resistivity as salts are diluted or flushed through the interconnected pockets by melt water.

Furthermore the 2008 Arctic data, displayed in the plots in this chapter, indicate the following depth dependent behaviour. On the whole (i) the temperature increases; (ii) ice salinity follows a C-shaped curve; (iii) the calculated brine volume fraction 
increases; (iv) calculated values of brine salinity decrease and (v) computed brine resistivity values increase.

The temperature variation with depth is clearly dependent on the difference between the air temperature and the warm seawater, which is insulated by the ice. As such, while the ambient air temperature is lower than that of the water, the temperature of the ice increases with depth. The brine volume fraction increases with depth as newer ice forming at the bottom is more porous than that above. As described earlier, the salinity of the ice is high near the surface and base of the sea ice, with the highest salinity near the ice/water interface. The brine salinity, on the other hand, is generally lower at the bottom of the ice than at the top. The brine closer to the surface is in colder ice and as such must have a high salinity in order not to be frozen, whereas deeper in the ice the temperature is higher and the salinity of the brine need not be as high. This reasoning would also explain the observed increase in the resistivity of the brine as one goes deeper into the ice.

With the increase in air temperatures in mid May the upper regions of the ice cover begin to warm, and the temperatures measured in these regions begin to show a decreasing trend with depth (rather than an increasing trend). Decreasing temperature with depth leads to increasing brine salinities and decreasing brine resistivity with depth. The colder ice at depth within the upper regions of the ice cover will contain brine pockets of more concentrated brine while in the warmer ice nearer the surface the brine inclusions are likely to be more dilute. Hence, this change in the temperature profiles corresponds quite nicely with the observed change to increasing sea ice salinity in the upper regions of the ice cover.

Over the measurement period of the 2009 Antarctic data the properties of the ice, as shown in the plots in this chapter, indicate that in general: (i) the temperature increases; (ii) calculated values of brine salinity decrease and (iii) computed brine resistivity values remain mostly unchanged.

In comparison to the Arctic data the recorded temperatures are lower leading to higher brine salinity, lower brine resistivity and the slightly lower brine volume fractions being calculated. The salinity of the ice is similar for both sites, and despite the differing values the general trends appear to be similar for all properties. 
Table 3.3 and Table 3.4 below display a summary of the measurements made and their average values for each of the data sets obtained over the two measurement periods $7^{\text {th }}$ Apr to $17^{\text {th }}$ Jun, 2008 (Table 3.3) and $11^{\text {th }}$ Nov to $21^{\text {st }}$ Nov, 2009 (Table 3.4). 


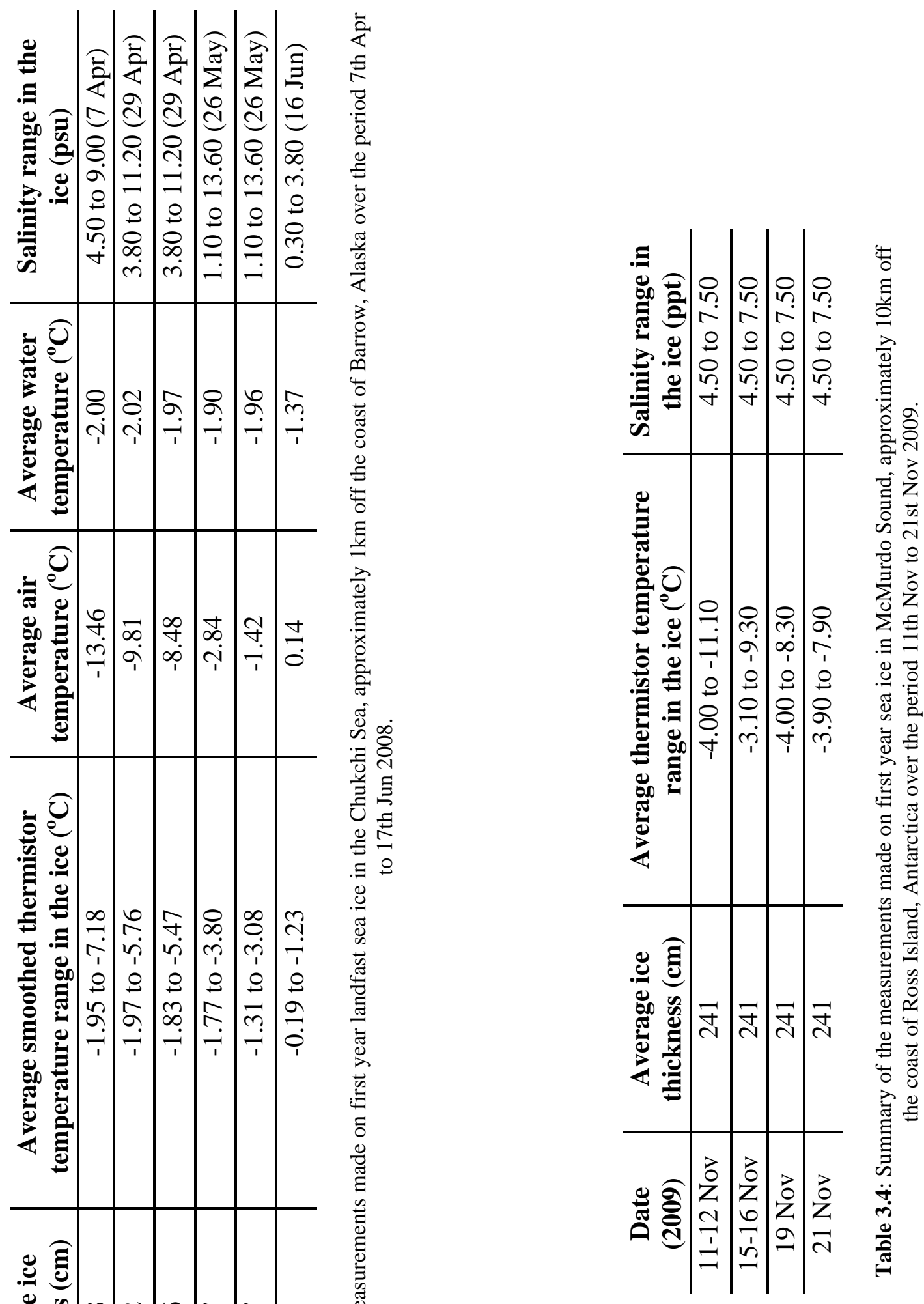




\section{Chapter 4 \\ Obtaining resistivity models}

For each measurement set of the geometric mean or horizontal resistivity the recorded data files (see Table 3.1 and Table 3.2 in Chapter 3) were combined and processed to remove any repeat or bad data, such as zero resistance being measured or anomalous readings caused by a suspected bad connection. Some of the measured resistance values were negative, implying that for the given current electrodes $\mathrm{C} 1$ and $\mathrm{C} 2$, the potential at P1 was actually lower than at P2. These values were made positive by simply changing the polarity of the measurement i.e. swapping the designated positions of the two potential electrodes between which the measurements were made. After being processed the data sets consisted of hundreds to thousands of resistance measurements (as noted in Table 3.1 and Table 3.2 in Chapter 3). In order to obtain the resistivity structure of the ice it is necessary to invert these data. To this end, the inversion program RES3DINV by Geotomo software was used to produce $3 \mathrm{D}$ models of the resistivity structure of the sea ice.

\subsection{Data inversion ${ }^{\dagger}$}

In general resistivity inversions the medium is parameterised by dividing it into a number of model blocks of constant resistivity. The electrical response of this parameterised medium is a non-linear function of the model parameters. However, given an initial guess for model parameters, the nonlinear least-squares problem can be linearised by using a first-order Taylor expansion as follows

$$
\underline{g}=\underline{\underline{J}} \underline{d}
$$

where

- $\underline{g}$ is the discrepancy vector or a vector which contains the differences between the measured data $\underline{y}$ and the model data $\underline{x}(\underline{g}=\underline{y}-\underline{x})$

- $\underline{d}$ is the model perturbation vector or a vector containing the corrections to the model parameters $\underline{m}$

\footnotetext{
${ }^{\dagger}$ Most of this section is derived from the following sources; Sasaki (1989), Sasaki (1992) and Loke (2004). Sasaki (1989) and Sasaki (1992) both contain sections on the theory of smoothness-constrained inversions. The 2004 tutorial by Loke provides basic inverse theory, particularly in regards to the programs RES2DINV and RES3DINV.
} 
- $\underline{\underline{J}}$ is the Jacobian matrix of the partial derivatives of the model data with respect to the model parameters. The components of this matrix are given by $J_{i j}=\frac{\partial x_{i}}{\partial m_{j}}$, that is the change in the $i$ th model response due to the change in the $j$ th model parameter.

\subsubsection{Least-squares optimisation method}

In the least-squares optimisation method, the aim is to minimise the sum of the squares of the discrepancy vector $E=\underline{g}^{T} \underline{g}$. The following Gauss-Newton equation is used to determine the change in the model parameters that would give this minimum error

$$
\underline{\underline{J}}^{T} \underline{\underline{J}} \underline{d}=\underline{\underline{J}}^{T} \underline{g}
$$

where $\underline{\underline{J}}^{T}$ is the transpose of the Jacobian matrix $\underline{\underline{J}}$

After calculating the parameter change vector a new model is obtained by adding the parameter changes to the current model $\rightarrow \underline{m}_{k+1}=\underline{m}_{k}+\underline{d}$.

\subsubsection{Marquardt-Levenberg modification}

In practice, the simple least-squares equation (4.1) above is rarely used by itself in geophysical inversion. In some situations the matrix product $\underline{\underline{J}}^{T} \underline{\underline{J}}$ might be singular, and thus the least-square equation does not have a solution for $\underline{d}$. Another common problem is that the matrix product $\underline{\underline{J}}^{T} \underline{\underline{J}}$ is nearly singular. This can occur if a poor initial model, that is very different from the optimum model, is used. The parameter change vector $\underline{d}$ can have components that are too large such that the new model $\underline{m}_{k+1}$ might have values that are not realistic. One common method to avoid this problem is the MarquardtLevenberg modification to the Gauss-Newton equation, (4.1).

The approximate misfit of the corrected model to the observed data is given by

$$
\underline{\Phi}_{1}=(\underline{g}-\underline{J} \underline{d})^{T}(\underline{g}-\underline{J} \underline{d})
$$

The change in model parameters $(\underline{d})$ is sought in such a way that the approximate misfit is minimised under the constraint that $\underline{d}^{T} \underline{d}$ has some value. Such minimisation leads to 


$$
\left(\underline{\underline{J}}^{T} \underline{\underline{J}}+u \underline{\underline{I}}\right) \underline{d}=\underline{\underline{J}}^{T} \underline{g}
$$

where

- $u$ is a Lagrange multiplier (or damping factor) which effectively constrains the range of values that the components of $\underline{d}$ can take.

- $\underline{\underline{I}}$ the identity matrix

The Marquardt-Levenberg method modification seen in (4.2) minimises a combination of the magnitude of the discrepancy vector $\underline{g}$ and the parameter change vector $\underline{d}$.

The estimated parameter changes in $\underline{d}$ are used to update the model and a sequence of iterations is performed until the misfit between the measured data and the model data is reduced to a desirable level, or until there are no significant differences in the estimates between successive iterations. The RMS misfit is given by

$$
\text { RMS misfit }=\sqrt{\underline{g}^{T} \underline{g} / N}
$$

where

- $N$ is the number of data points

\subsubsection{Smoothness-constrained least-squares method}

For a large number of model parameters (i.e. 2D and 3D modelling) the model produced by the Marquardt-Levenberg method can have an erratic resistivity distribution with spurious high or low resistivity regions. To overcome this problem, the Gauss-Newton least-squares equation is further modified. Such that the square of the spatial changes, or roughness of the model resistivity values is minimised.

It is necessary to impose some form of constraint on the perturbation vector $(\underline{d})$ in order to stabilise the inversion process. The approach of Sasaki (1992) is to estimate the perturbation vector that will minimise the misfit, subject to the constraint that the smoothness of the model has some constant value.

The degree of roughness (the reciprocal of smoothness) about the $j$ th block may be defined as

$$
r_{j}=\left(d_{j}^{E}+d_{j}^{W}+d_{j}^{N}+d_{j}^{S}-4 d_{j}\right) / 4
$$


where the superscripts $E, W, N$ and $S$ refer to the four immediate neighbours of the $j$ th block.

This equation can be re-written in matrix form as

$$
\stackrel{r}{=}=\underline{\underline{C}} \underline{d}
$$

where $\underline{\underline{C}}$ is a 2 D Laplacian operator whose elements are either $-1,1 / 4$ or 0 .

The total roughness of the model is given by

$$
\underline{\Phi}_{2}=\underline{\underline{r}}^{T} \underline{\underline{r}}=(\underline{\underline{C}} \underline{\underline{d}})^{T}(\underline{\underline{C}} \underline{\underline{d}})
$$

The objective function to be minimised is then expressed as

$$
\underline{\Phi}=\underline{\Phi}_{1}+u \underline{\Phi}_{2}=(\underline{g}-\underline{J} \underline{d})^{T}(\underline{g}-\underline{J} \underline{d})+u(\underline{\underline{C}} \underline{d})^{T}(\underline{\underline{C}} \underline{d})
$$

From this relation the method of Lagrange multipliers gives a solution of

$$
\begin{aligned}
& \underline{d}=\left(\underline{\underline{J}}^{T} \underline{\underline{J}}+u \underline{\underline{C^{T}}} \underline{\underline{C}}\right)^{-1} \underline{J}^{T} \underline{g}=\left(\underline{\underline{J}}^{T} \underline{\underline{J}}+u \underline{\underline{F}}\right)^{-1} \underline{J}^{T} \underline{g} \\
& \rightarrow\left(\underline{\underline{J}}^{T} \underline{\underline{J}}+u \underline{\underline{F}}\right) \underline{d}=\underline{\underline{J}}^{T} \underline{g}
\end{aligned}
$$

where

- $\underline{\underline{C}}^{T} \underline{\underline{C}}=\underline{\underline{F}}=\underline{f}_{x} \underline{f}_{x}^{T}+\underline{f}_{z} \underline{f}_{z}^{T}$ is the flatness filter and is used to constrain the smoothness of the perturbations to the model parameters to some constant value.

- $f_{x}=$ horizontal flatness filter

- $f_{z}=$ vertical flatness filter

This formulation constrains the change in the model resistivity values $(\underline{d})$, to be smooth but does not guarantee that the resistivity values change in a smooth manner.

\subsubsection{Directly smoothing the model resistivities}

This form of the Gauss-Newton equation (4.3) can be further modified so that the smoothness constraint is applied directly to the model resistivity values giving

$$
\left(\underline{\underline{J}}^{T} \underline{\underline{J}}+u \underline{\underline{F}}\right) \underline{d}=\underline{\underline{J}}^{T} \underline{g}-u \underline{\underline{F}} \underline{\underline{m}}
$$

This method tends to produce a model with a smooth variation of resistivity values. 


\subsubsection{Robust inversion method}

The approach of equation (4.4) is acceptable if the actual subsurface resistivity varies in a smooth and gradational manner. However, in some cases, the subsurface structure consists of a number of regions that are internally almost homogeneous but with sharp boundaries between different regions. For such cases the inversion formulation (4.4) can be modified so that it minimises the absolute changes in the model resistivity values. This can sometimes give significantly better results.

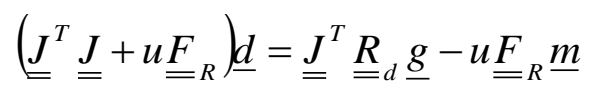

where

- $\underline{\underline{F}}_{R}=\underline{\underline{C}}^{T} \underline{\underline{R}}_{m} \underline{\underline{C}}$ is the modified flatness filter, and

- $\underline{\underline{R}}_{m}$ and $\underline{\underline{R}}_{d}$ are weighting matrices introduced so that different elements of the data misfit and model roughness vectors are given equal weights in the inversion process.

This is known as a robust or blocky inversion method. 


\subsection{RES3DINV - Geotomo Software}

In this study the data are inverted using RES3DINV - Rapid 3D resistivity and IP inversion produced by Geotomo software. This inversion program is based upon a smoothness-constrained least-squares method (Lytle and Dines, 1980, Sasaki 1989, 1992, deGroot-Hedlin and Constable 1990, Loke and Barker 1996b), and works by finding the smoothest change to the model for which the residual error lies within a desired tolerance. Such models are considered to have only the structural features demanded by the data and produce few spurious features (Sasaki 1989).

The inversion routine uses the following basic equation, as given in section 4.1.3 above

$$
\left(\underline{\underline{J}}^{T} \underline{\underline{J}}+u \underline{\underline{F}}\right) \underline{d}=\underline{\underline{J}}^{T} \underline{g}
$$

where the parameters are as previously defined.

The inversion program divides the subsurface into a number of small rectangular blocks (in the basic arrangement the corners of the blocks are defined by the unit separation of the electrodes), and attempts to determine the resistivity values of the blocks so as to minimise the difference between the calculated and observed apparent resistivity values.

To start this process RES3DINV produces an initial model $\underline{m}_{0}$, forward modelling is then used to calculate the theoretical apparent resistivity values for this model. This model data, $\underline{x}$ is then compared with the measured data, $\underline{y}$ and the discrepancy vector, $\underline{g}=\underline{y}-\underline{x}$ obtained. The Jacobian matrix $\left(J_{i j}=\partial x_{i} / \partial m_{j}\right)$ is calculated from the partial derivatives of the model data with respect to the model parameters (i.e. the resistivities in the blocks of the current model). The Jacobian along with the discrepancy vector $(\underline{g})$ are used to find the model perturbation vector $(\underline{d})$ by introducing some form of constraint (minimising misfit or roughness etc.) (see section 4.1). After the parameter change or model perturbation vector has been found a new model $\left(\underline{m}_{k+1}\right)$ is obtained by adding the parameter changes to the current model $\left(\underline{m}_{k}\right)$, i.e. $\underline{m}_{k+1}=\underline{m}_{k}+\underline{d}$.

A measure of the difference between the modelled and observed resistivity values is given by the root-mean-squared (RMS) error. However the model with the lowest possible RMS error can sometimes show large and unrealistic variations in the model

\footnotetext{
$\$$ The reader is directed to the RES2DINV and RES3DINV user manual and the 2004 tutorial by Loke for further details of the RES3DINV inversion process.
} 
resistivities and might not always be the best model from a geological perspective. In general the most prudent approach is to choose the model at the iteration after which the RMS does not change significantly.

\subsubsection{Data files}

The RES3DINV program reads files which contain information on the size and arrangement of the model grid, electrode arrangement, the measured data and any added features such as fixed regions and topography etc (see Appendix II)

\section{Grid size}

The length of the model blocks in the $x$ and $y$ directions were set to $0.05 \mathrm{~m}$, half the electrode separation. The layer thickness or the length of the model block in the $z$ direction remains at the electrode separation of $0.1 \mathrm{~m}$. This should provide a detailed model of the internal resistivity structure of sea ice, while not using a grid that is too fine for the resolution of the measurement equipment. Applying this grid size produced a sensible sequence of resistivity models over the measurement period. However, for inversion of data gathered in the Arctic on 7-9 April 2008 it was found that having a grid size of $0.05 \mathrm{~m}$ produced a model with lower than expected resistivities, in fact lower than all other data sets except the 16-17 June (by which stage the ice was in full melt). The resistivity of the model could be increased by either increasing the grid size to the electrode separation $(0.1 \mathrm{~m})$ or removing the fixed region (see below). As we know there is seawater below the ice cover the fixed region should stay as part of the model. Thus, in order to obtain what is judged as a more realistic resistivity structure, the best solution appears to be to increase the grid size to $0.1 \mathrm{~m}$ for this data set. Further evidence of the unsuitability of a $0.05 \mathrm{~m}$ grid in the inversion of the 7-9 April data is seen in the larger error in the fit of this model to the measured data.

\section{Including fixed regions}

The RES3DINV 3D inversion program allows the user to input any regions of known resistivity as a fixed region in the input files it reads. In the case of modelling the resistivity structure of sea ice this option is useful, as it allows the presence of the seawater beneath the ice to be included in the model. A damping factor weight allows control of the degree to which the inversion program can change the resistivity of the 
'fixed' region. If a damping factor weight of 1 is used, the resistivity of the region is allowed to change to the same extent as other regions of the subsurface model. If a relatively large value is used (e.g. 10) the change in the resistivity of the region would be very small. Such a large value should only be used if the resistivity and shape of the region is accurately known.

Fixed regions of $0.4 \Omega \mathrm{m}$, at the relevant depths, were used in the modelling of the 2008 Arctic data in order to approximate the seawater under the ice, thus providing a more realistic model in terms of the seawater/ice interface. For the 16-17 June 2008 data from the Arctic a fixed region of high resistivity $(1,000,000 \Omega \mathrm{m})$ was also added to the surface of the model to represent the electrode strings protruding from the ice cover due to surface ablation. During the Antarctica measurements in 2009 the ice thickness was always greater than the length of the electrode strings, thus the seawater/ice interface was not observed and no fixed regions were used in the modelling. The average thickness of the ice at the sites was obtained from the mass balance sites. These thickness values were used to determine the relevant depths for the fixed region. A damping factor weight of 2 was decided upon to give sufficient steadiness to the fixed region, thus ensuring a physically real model while not imposing the region as an absolute part of the model (especially since the exact shape of the seawater/ice interface is unknown).

\subsubsection{Inversion parameters}

\subsubsection{Resistance or apparent resistivity as inversion variable}

If the data are recorded as resistance values then RES3DINV gives the option of inverting the data set using apparent resistivity or directly using the resistance values from the file. Using resistance values directly in the inversion has the advantage that readings that have an apparent resistivity value that does not exist or is negative are allowed to be used. To convert from the measured resistance values to apparent resistivity the inversion program estimates the potential per unit input current, which would be measured by each electrode configuration in the data set.

Two issues arose with attempts to use the apparent resistivity as the inversion variable while inverting the geometric mean resistivity data. The first issue was that it was 
necessary to remove data points for which RES3DINV calculated low potentials, this led to a significant decrease in the size of data sets. It is possible this decrease would give a model with lower resolution. Furthermore it is not unreasonable to expect low apparent resistivity values in the highly conductive seawater beneath the ice and these values should be retained in the inversion model. Another issue for several data sets was that the calculated apparent resistivity values were too small. This caused the program to produce a warning message and stop the inversion. This could be overcome if apparent resistivity rather than the log of the apparent resistivity was used as the data parameter, however, it is then not possible to change the data inversion from the robust to the standard option (see section 4.2.2.7).

As the recorded data are resistances it makes sense to directly use this as the inversion variable and it avoids issues with having to remove data points with low potentials or producing calculated apparent resistivity values that are too small for the inversion to continue. However, if these issues can be avoided (as seems to be the case for the horizontal resistivity), using either variable should be equally acceptable.

\section{Limiting the range of model resistivity}

If apparent resistivity is used as the inversion variable the program can limit the range of resistivity values the inversion subroutine will give. The limits can be entered by the user as multiples of the average model resistivity value of the previous iteration. The program uses 'soft' limits that allow the actual model resistivity values to exceed the limits to a certain degree. However, this option will avoid extremely small or large model resistivities that are physically unrealistic. As the data set gathered for this research has a wide range of resistivities from tenths of a ohm meter to 1000's of ohm meters it would not be prudent to constrain the model by limiting the resistivity values.

\subsubsection{Initial model}

One of two different types of initial model can be selected. The default initial model is a homogeneous half space. The resistivity of the half space is often calculated from the average value of the measured apparent resistivities (Loke and Barker, 1996a). For areas with high resistivity contrasts it is suggested that a better initial model might be obtained by using an approximate inverse method. However, when the effect of the type of initial model was investigated there did not appear to be any difference in the final model produced (see Figure 4.1 below). 


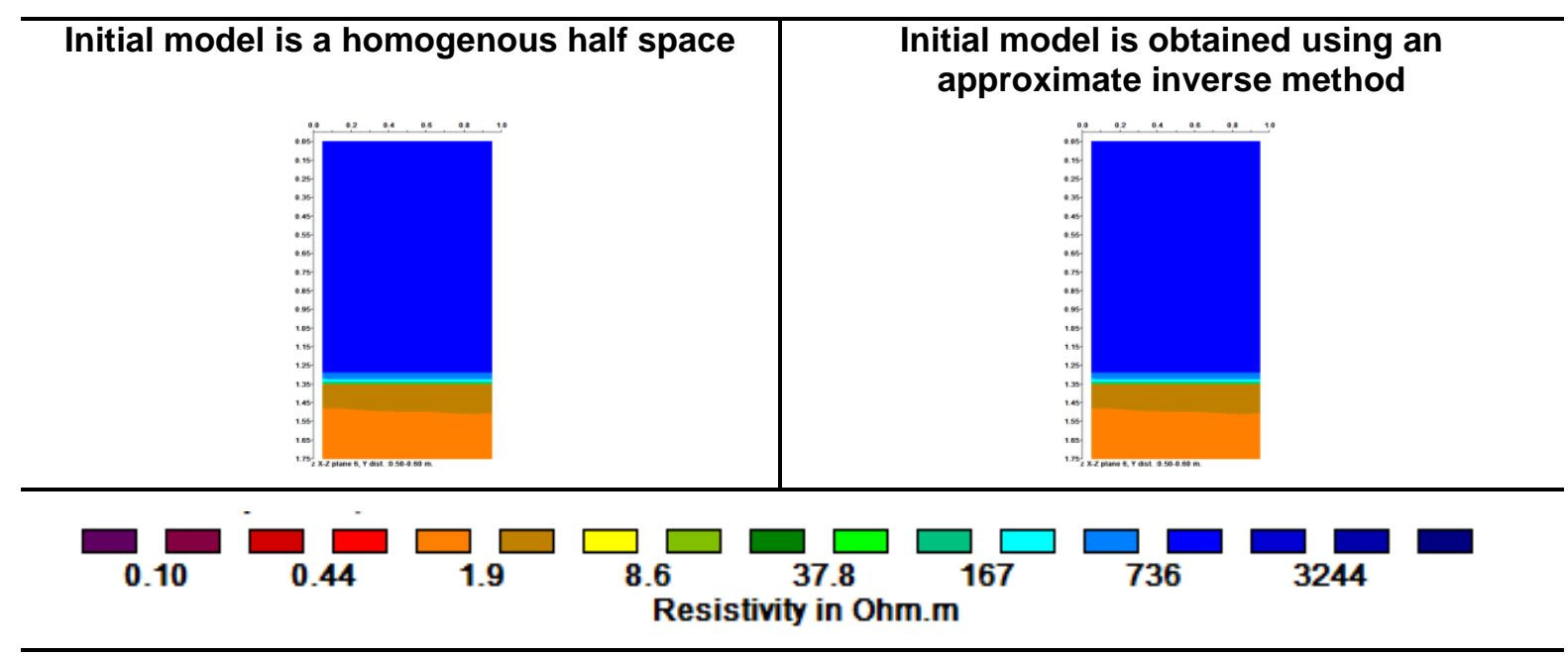

Figure 4.1: Comparison of models produced using a homogeneous half space as initial model or using the approximate inverse method to obtain the initial model. Displayed are X-Z sections through resistivity model at $y$ distances of $0.5 \mathrm{~m}-0.6 \mathrm{~m}$

\subsubsection{Forward modelling method}

The forward modelling subroutine is necessary to calculate the theoretical apparent resistivity values for the model produced by the inversion, so that the model can be compared to the measured resistivities. RES3DINV allows the use of either the finitedifference or finite element-method to calculate these apparent resistivities.

The finite difference method replaces the derivatives appearing in the differential equation by finite differences that approximate them. The finite element method finds approximate solutions of partial differential equations by either eliminating the differential equation, or approximating the partial differential equations by ordinary differential equations. The finite difference method is an approximation to the differential equation while the finite element method is an approximation to its solution. The finite-element method is significantly slower than the finite-difference method.

If the data set contains topography the default is the finite-element method while if the data set contains no topography the default is the finite-difference method. With no topography in the data sets, the faster finite-difference method was used to calculate the model resistivities.

\subsubsection{Damping factor}

\section{Initial and minimum damping factors}

The RES3DINV inversion program allows the user to set a value for the initial damping factor ( $u$ in equation (4.6)) If the data are noisy the initial value should be large (e.g. 
0.3). The inversion subroutine will generally reduce the damping factor after each iteration. However, a minimum limit for the damping factor is required to stabilise the inversion process, this value should be set to about $1 / 5$ to $1 / 15$ of the initial value. Default values of initial -0.15 and minimum -0.01 damping factors are used.

\section{Optimise damping factor}

Instead of automatically decreasing the damping factor after each iteration, the 'optimise damping factor' option allows the program to look for an optimum damping factor. The time taken per iteration is longer, as it is necessary to solve the least-squares equation more than once, but the program may complete fewer iterations before converging. When this option is selected the program will attempt to find the optimum damping factor which gives the lowest RMS error in each iteration. When using this option it is important to remember that the model with the lowest RMS error is not always the best model, particularly for very noisy data sets. The damping factor was optimised when inverting the data.

\subsubsection{Flatness filter}

If the resistivity structure is known to have anomalies elongated in the vertical (e.g. dykes and faults) or horizontal (e.g. sedimentary layers and sills) directions then the vertical to horizontal flatness filter ratio can be increased or decreased respectively. The vertical and horizontal flatness filters are the $f_{z}$ and $f_{x}$ components of the flatness filter $\underline{\underline{F}}$ from equation (4.6) above. Changing the ratio of these values allows fine-tuning of the smoothness-constraint to emphasize vertical or horizontal structures in the inversion model. The inversions carried out for this research use a ratio of vertical to horizontal filter weights, left at the default value of 1 (i.e. no preference for horizontal or vertical structure).

\subsubsection{Directly smooth model resistivities}

In section 4.1.4 it was shown that the smoothness constrained least-squares form of the Gauss-Newton equation (4.6) can be further modified to

$$
\left(\underline{\underline{J}}^{T} \underline{\underline{J}}+u \underline{\underline{F}}\right) \underline{d}=\underline{\underline{J}}^{T} \underline{g}-u \underline{\underline{F}} \underline{\underline{m}}
$$

where the parameters are as previously defined.

Selecting the option to use this equation in RE3DINV leads to a smoothness constraint being applied to the model resistivity values as well as to the model perturbation vector. 
This will usually produce a model with a larger apparent resistivity RMS error but a smooth variation in the resistivity values.

If the model inversion was set to the robust model constrain (see section 4.2.2.7) it seemed that the program forced the model resistivities to be directly smoothed - this option could not be unselected. The effects of directly smoothing the model resistivities or not can be seen in Figure 4.2 below.

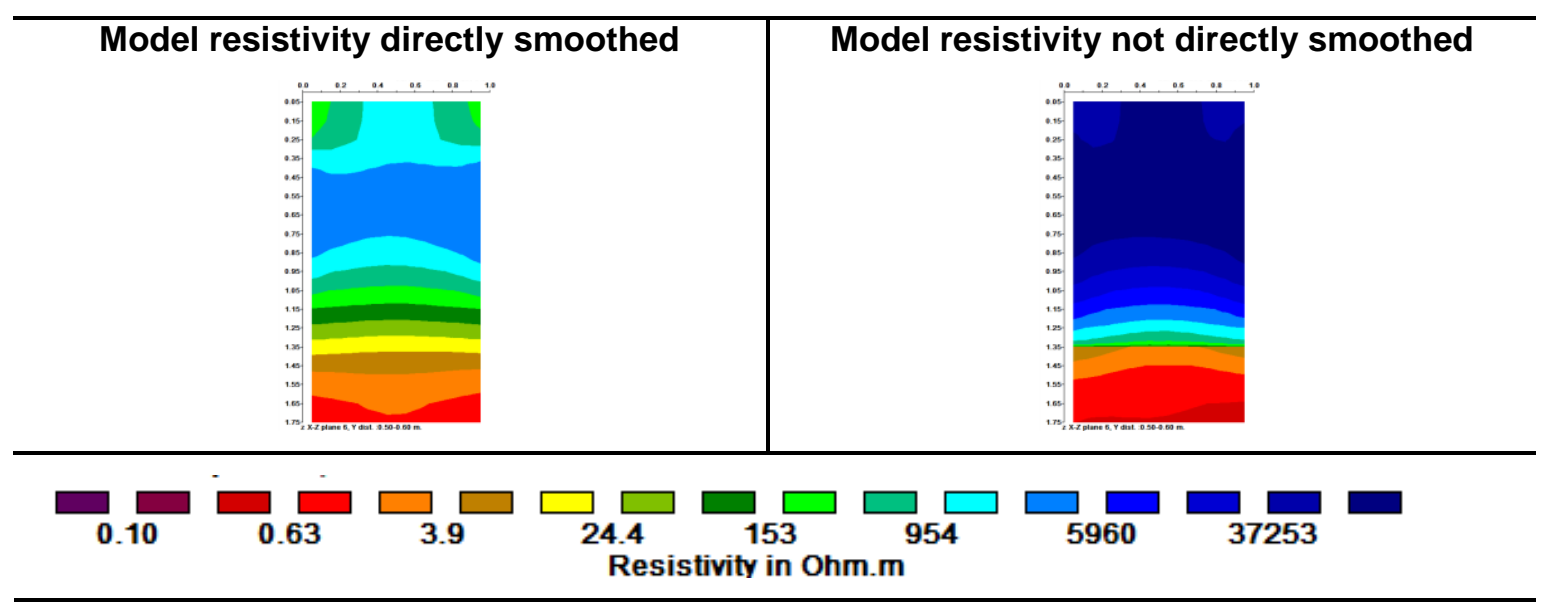

Figure 4.2: Effect of directly smoothing the model resistivity on the inversion models. Displayed are $\mathrm{x}-\mathrm{Z}$ sections through resistivity model at y distances of $0.5 \mathrm{~m}-0.6 \mathrm{~m}$.

When the model resistivities are not directly smoothed the robust/standard inversions produced models which seem to give very large and unrealistic horizontal apparent resistivity values (see Figure 4.2) of the order of 100,000's of ohm meters. Hence when inverting the data the model resistivities are directly smoothed.

\subsubsection{Robust inversion method}

Directly smoothing the model resistivity values is acceptable if the actual subsurface resistivity varies in a smooth manner. However, the subsurface geology could be made up of a number of regions that are internally homogeneous but have sharp boundaries. In such cases the inversion formulation can be further modified (as seen in section 4.1.5) so that it minimises the absolute changes in the model resistivity values giving a robust inversion method.

$$
\left(\underline{\underline{J}}^{T} \underline{\underline{J}}+u \underline{\underline{F}}_{R}\right) \underline{d}=\underline{\underline{J}}^{T} \underline{R}_{d} \underline{g}-u \underline{\underline{F}}{ }_{R} \underline{m}
$$

with the parameters as defined previously. 
For both the model and data inversions RES3DINV gives the option of choosing either the standard least squares constraint (expression (4.6)) or a robust constraint (expression (4.8)). The standard least squares method attempts to minimise the square of the difference between measured and calculated apparent resistivity values. On the other hand the robust method down weights values that are significantly away from where the majority are distributed (i.e. it removes outliers that could influence the resulting inversion), is less sensitive to very noisy datum points and tends to produce models with sharp boundaries but that might give a higher RMS value.

Setting the data inversion to the robust constraint, the inversion program will attempt to minimise the absolute difference between the measured $(\underline{y})$ and calculated $(\underline{x})$ apparent resistivity values. In other words minimise the discrepancy vector, $\underline{g}$ (see section 4.1), rather than the sum of the squares of the discrepancy vector. There is a cut-off factor which controls the degree to which this robust data constraint is used. If a value of 0.05 (default value) is used the effect of data points, where the differences in the measured $(\underline{y})$ and calculated $(\underline{x})$ apparent resistivity values are much greater than 5 percent, will be greatly reduced.

For the model inversion the robust constraint will attempt to minimise the absolute spatial changes in the model resistivity values. That is it will constrain the changes that can be made to the model through the perturbation vector $\underline{d}$. As with the robust data constraint there is a cut-off factor which controls the degree to which this constraint is used. If a large value is used, for example 1.0, the result is essentially that of the conventional smoothness-constrained least-squares inversion method (see equation (4.6)). If a very small value is used, for example 0.001, the result is close to the true robust constrained inversion method. The default value for the cut-off is 0.01 , as shown in Figure 4.3 below, decreasing the robust model constraint cut-off from 0.01 to 0.001 has little effect on the model. Thus there is no reason to further decrease the cut-off from the default value in attempts to return a result closer to the true robust constrained inversion method 


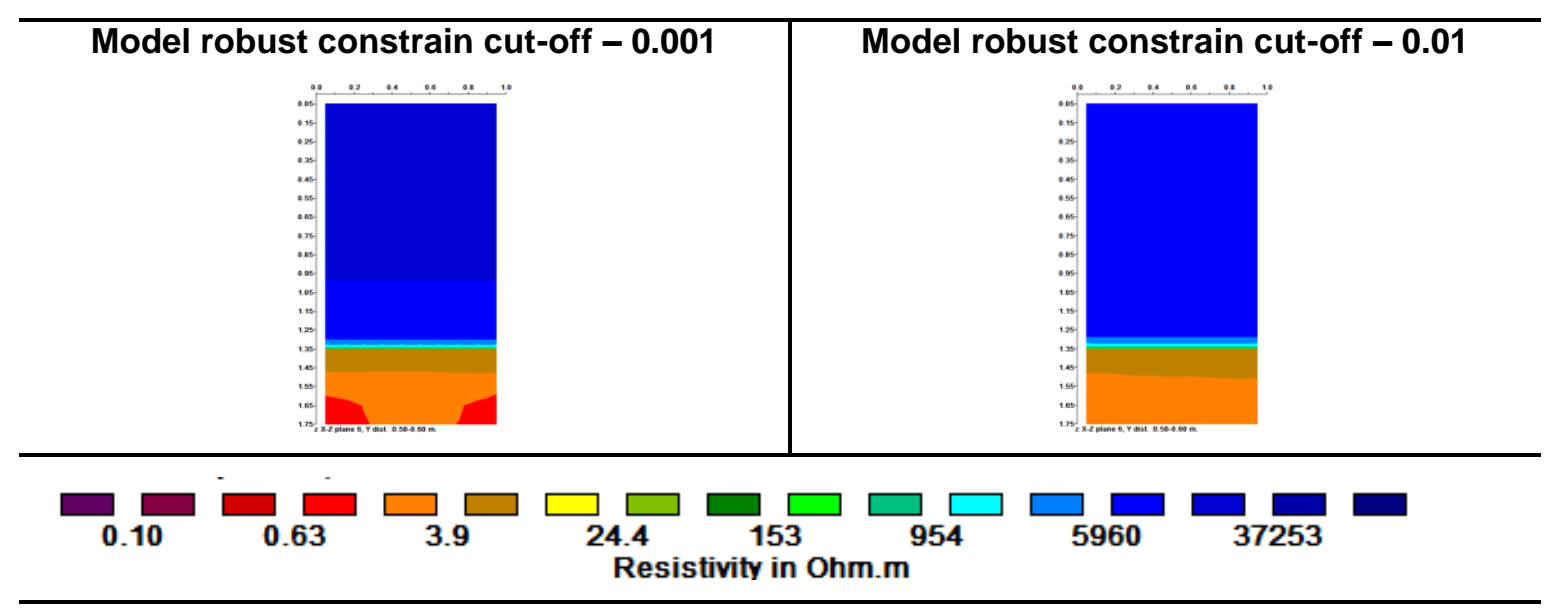

Figure 4.3: Effect of different model robust constraint cut-off values. Displayed are $\mathrm{x}-\mathrm{z}$ sections through resistivity model at y distances of $0.5 \mathrm{~m}-0.6 \mathrm{~m}$.

When testing the best inversion parameters to use during modelling it was discovered that while using resistance as the inversion variable data inversion could not be set to use the standard least squares constraint.

The robust constraint is used on both the data and model inversion with cut-off values set to the defaults of 0.05 and 0.01 respectively.

\subsubsection{Solving the least squares equation}

The RES3DINV 3D inversion program provides the user with a choice of two different methods of solving the least squares equation (i.e. equation (4.6), (4.7) or (4.8)). The program default uses the standard Gauss-Newton least squares method. This method calculates an exact solution of the least squares equation and involves recalculating the Jacobian matrix of partial derivatives after every iteration. This is time consuming, but in areas with resistivity contrasts of greater than 10:1 recalculating the Jacobian matrix produces models with boundaries which are much sharper and generally gives slightly better results. To reduce the inversion time, an alternative method that calculates an approximate solution of the least squares equation using the incomplete Gauss-Newton method can be used. The user can set the accuracy of the solution, for most data sets an accuracy of about $1 \%$ to $2 \%$ seems to provide a solution that is almost the same as that obtained by the standard Gauss-Newton method.

As this project is looking at a medium where there is a large range of resistivity values varying from tenths of ohm meters, in sea water, to thousands of ohm meters in pure ice, and the possibility of sharp boundaries (e.g. the seawater/ice interface) the default of the Gauss-Newton method is used for the inversions. 


\subsubsection{Resistivity change: optimum step size}

In order to find the optimum step size for the change in the resistivity of the model blocks the inversion program gives the option of performing a line search using quadratic interpolation. This will require at least one forward modelling computation per iteration. In some cases this extra computation could be worthwhile if it reduces the number of iterations needed to bring the RMS value down to an acceptable level. For the first two iterations the program will always carry out a line search in an attempt to find the optimum step size. The line search method can also estimate the expected change in the RMS error and if the change is too small, it might not be worthwhile to proceed with the line search. For this research inversions were carried out using the default settings of completing a line search on every iteration that had a minimum change in the RMS error of $0.2 \%$.

\subsubsection{Converging the inversion}

\section{Maximum number of iterations}

RES3DINV allows the user to set the maximum number of iterations for the inversion routine. The maximum has been left at the default value of 6 iterations, which should be sufficient for most data sets.

\section{Convergence limit}

The lower limit for the relative change in the RMS error between two iterations can also be set by the user. The program uses the relative change in RMS error, rather than an absolute RMS value, so as to accommodate different data sets with different degrees of noise present. By default a value of $5 \%$ is used, that is if the change in RMS between two consecutive inversions is less than $5 \%$ the inversion process will end.

\subsubsection{Summary}

To obtain three dimensional models of the resistivity structure of sea ice, the measured resistance data are input into the inversion program RES3DINV and the following inversion parameters (summarized in Table 4.1) used:

Grid size (section 4.2.1)

The size of the model blocks in the $x$ and $y$ directions are set to $0.05 \mathrm{~m}$ or half the electrode separation. While the vertical size of the model blocks is set to the electrode 
separation of $0.1 \mathrm{~m}$. This should provide a detailed model without being too fine for the resolution of the measurement equipment.

\section{Fixed region (section 4.2.1)}

Using the average thickness of the ice a region of low resistivity $(0.4 \Omega \mathrm{m})$ was added to the base of the initial model. The aim was to produce a more physical model by incorporating the presence of seawater, while still allowing the model to change the resistivity values. Thus a fixed region was added and a damping factor weight of two selected.

Resistance as inversion variable (section 4.2.2.1)

As the measurements made in the field were recorded as resistances and there were issues with setting some of the inversion parameters, when apparent resistivity was used as the inversion variable, it made sense to generally use the resistance values directly.

Initial model (section 4.2.2.2)

A homogeneous half space was used as the initial model for the inversion process. However, there seems to be no difference if the approximate inverse method is used.

\section{Forward modelling method (section 4.2.2.3)}

The finite difference method is used during the forward modelling stage to calculate the theoretical apparent resistivities for the models. This method is quicker than the finite element method.

\section{Optimising the damping factor (section 4.2.2.4)}

The inversion program is set to find the optimum damping factor when solving the least squares inversion equation. This produces models with the lowest RMS error.

\section{Directly smooth model resistivities (section 4.2.2.6)}

If the robust model inversion is used the program forces the model resistivities to be directly smoothed. It was also observed that not directly smoothing the model resistivities produces models with extremely high horizontal resistivity. Therefore the model resistivities are directly smoothed during the inversion process.

\section{Robust inversion method (section 4.2.2.7)}

The robust data constraint is set to the default of 0.05 this means that the effect of values of the discrepancy vector that are greater than $5 \%$ are reduced. The robust model 
constraint is set to the default value of 0.01. A small constraint value is closer to the robust inversion while a large value is closer to the standard inversion. Setting the model inversion to the robust method means the chance of producing a perturbation vector that is too large is reduced.

Solving the least squares equation (section 4.2.2.8)

The Gauss-Newton least squares method is used to solve the least squares equation as it calculates an exact solution. Additionally, using this method provides better results than the incomplete method, when the region being modelled has areas with resistivity contrasts of greater than 10:1. Furthermore the process of recalculating the Jacobian matrix for each iteration produces sharper boundaries.

Optimum step size (section 4.2.2.9)

To calculate the optimum step size to apply to the perturbation vector, RES3DINV is set to run a line search on every iteration that has a minimum change in the RMS error of $0.2 \%$.

Convergence limit and max number of iterations (section 4.2.2.10)

The maximum number of iterations to be performed by the inversion program is set to 6. The minimum change in RMS errors of two consecutive inversions is set to 5\%, if the difference gets below this value the inversion stops. These parameters should produce a satisfactory model for most data sets.

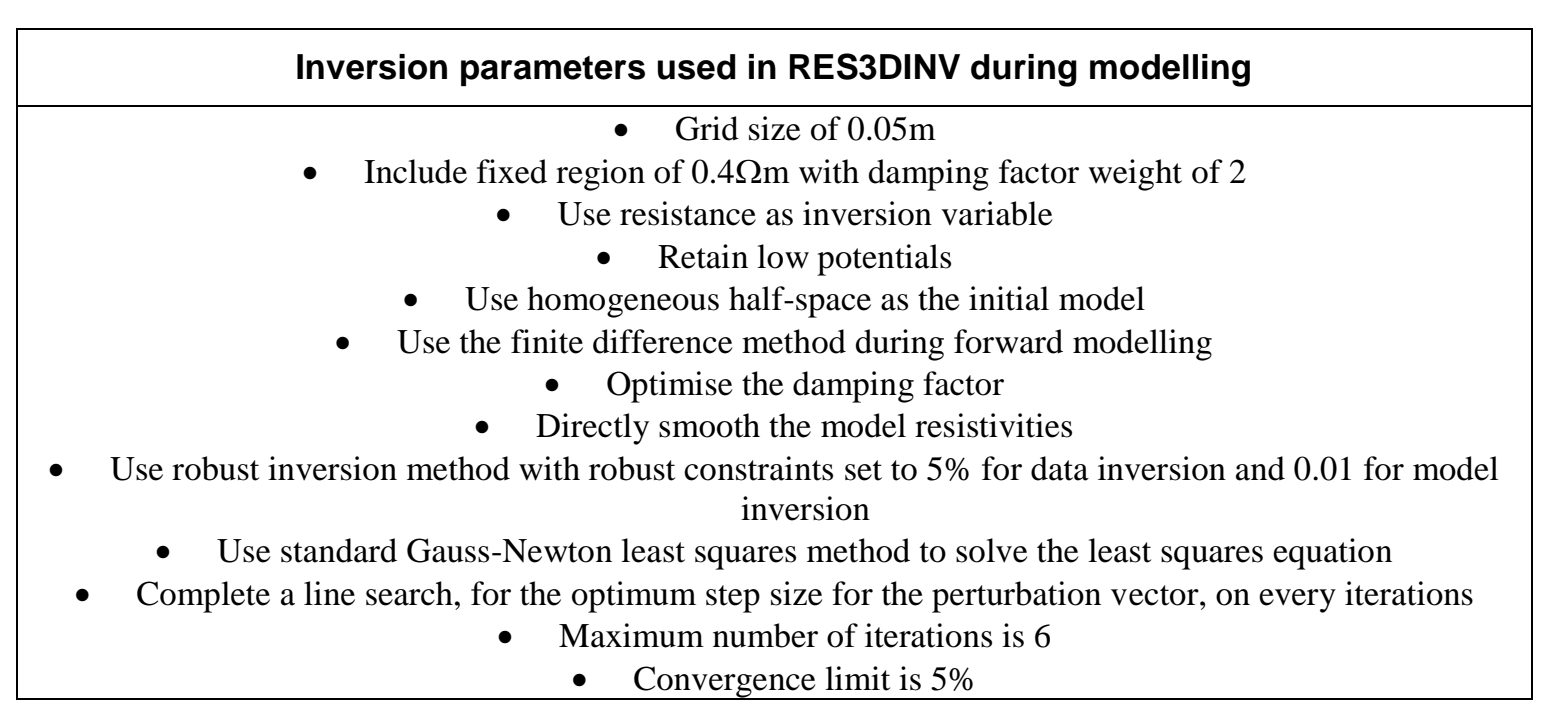

Table 4.1: Summary of inversion parameters. 



\section{Chapter 5 Analysing resistivity profiles}

This chapter displays and interprets models of the resistivity structure of first year sea ice from the Arctic and Antarctic.

The resistivity structure is obtained from 3D inversions of data gathered during electrical resistivity cross-borehole tomography measurements. As discussed in Chapter 3 , this technique uses four electrode strings inserted into boreholes which are positioned in the ice at the corners of a $1 \mathrm{~m}$ square.

Horizontal resistivities were obtained from measurements made between each of the six borehole pairs. Each measurement involving one current and one potential electrode in each borehole according to the criteria discussed earlier (section 2.2.1).

Measurements, aimed at determining the geometric mean resistivity structure, were obtained using a four electrode array with either; each of the electrodes at approximately the same depth in separate boreholes, or three electrodes at the same depth in separate boreholes and a remote surface electrode (section 2.2.2). However, due to the air/ice and sea/ice interfaces the geometric mean recovered by the inversion program is under-estimated and numerical modelling is required to retrieve a value of the coefficient of anisotropy and from this an estimate of the true resistivity (see section 2.3.2.2).

The vertical component of the bulk resistivity structure is then able to be calculated from the horizontal and geometric mean resistivities using the expression $\left(\rho_{v}=\rho_{m}{ }^{2} / \rho_{H}\right)$ that relates the three values.

Interpretation of the resistivity models in terms of changes in the microstructure of the ice was aided by temperature and salinity data, obtained from nearby the measurement sites, and calculated brine resistivities and brine volume fractions, as per the standard relations given by Stogryn (1971) and Cox and Weeks (1983) respectively. 


\subsection{Barrow, Alaska}

\subsubsection{8 measurements - Jones et al. (2010)}

As mentioned earlier (Chapter 3) six measurement sets were obtained, over April - June 2008 in first year sea ice off the coast of Barrow Alaska. Profiles through the resistivity models obtained from these measurements are displayed in this section.

Figure 5.1 displays both (a) sections through the models obtained from inversion of the geometric mean data (an underestimation of the resistivity value) and (b) the retrieved estimation of the true value of the geometric mean resistivity. As discussed earlier, synthetic data sets generated for different values of the anisotropy coefficient allow parameterization of the degree to which, for the observed ice thickness, $\rho_{m}^{M}$ is an underestimate of the true value of the geometric mean resistivity $\rho_{m}$. This parameterization takes the form of polynomials $F(\lambda)$ for each depth range in the 3D models. Such polynomials have been used, with the average values of $\rho_{H}$ and $\rho_{m}^{M}$ for each layer in the resistivity models, to estimate the actual variation of $\lambda$ with depth in the ice through numerical solution of expression (2.39). These values have then been used with the values of $\rho_{H}$ to determine the true values of $\rho_{m}$ through the ice. By the 16-17 Jun surface ablation was occurring, thus plots for this date start at a 'depth' of $0.2 \mathrm{~m}$.

Figure 5.2 shows (a) sections of the horizontal resistivity obtained from 3D inversion of the data, along with (b) the vertical resistivity structure - calculated from the horizontal and geometric mean resistivities and (c) the coefficient of anisotropy $(\lambda)$ - determined while retrieving the true $\rho_{m}$.

Figure 5.3 shows plots of the depth dependence of (a) the temperatures, measured at the UAF mass balance site using thermistor strings and (b) salinities, obtained by UAF via standard measurements on sea ice cores. The salinity of the ice was measured four times during our field work; on the 7 Apr, 29 Apr, 26 May and 16 Jun, and the salinity profiles for the ice during each of our measurement sets has been taken as that recorded on the nearest date to the resistivity measurement. As the temperature and salinity data aide the interpretation of the resistivity models, which use a discrete grid with vertical 
layers of $0.1 \mathrm{~m}$ depth, these data have been interpolated to give values at $0.1 \mathrm{~m}$ intervals starting at $0.05 \mathrm{~m}$ (the mid point of the $0-0.1 \mathrm{~m}$ layer). Additionally Figure $5.3 \mathrm{c}$ displays plots of brine volume fraction, calculated from standard relations as given in Cox and Weeks (1983). The ablation of the surface of the ice on the 16-17 Jun (as mentioned above), can be seen by the above zero temperatures recorded by the upper thermistors. This ablation means that the upper surface of the core used for the salinity measurements corresponds to temperatures at a recorded depth of approximately $0.2 \mathrm{~m}$. Furthermore the brine volume fraction plot takes $0 \mathrm{~m}$ to be the initial ice/air interface and thus starts at a depth of $0.2 \mathrm{~m}$ (with the surface brine volume fraction off the scale of the plot at $~ 32 \%$ ).

Finally, for each of the components of the bulk resistivity $\left(\rho_{H}, \rho_{m}, \rho_{V}\right)$ line plots of the average values with depth are shown in Figure 5.4, with all measurement sets shown on one plot - in order to allow better visualisation of the changes in time. This figure also includes a plot of the change in the coefficient of anisotropy over this measurement period. The standard deviations associated with the averaged values of $\rho_{H}$ and $\rho_{m}^{M}$ in each layer and the absolute misfit of the models to the observed data allow at least a superficial estimate of the uncertainties in $\rho_{\mathrm{H}}, \rho_{m}, \rho_{V}$ and $\lambda$. 


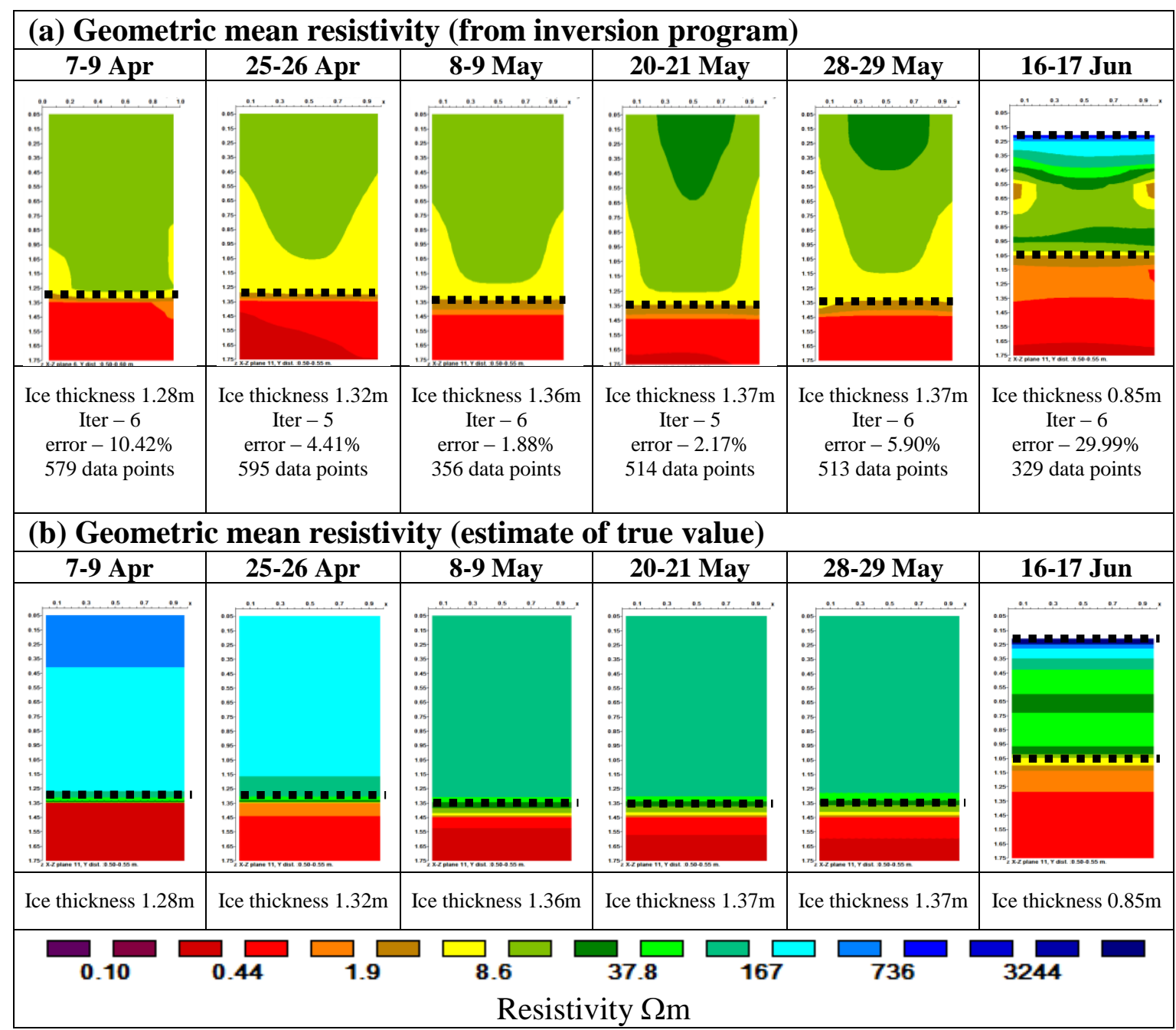

Figure 5.1: Vertical sections through models of the geometric mean resistivity, 2008 Arctic measurements. (a) the geometric mean resistivity obtained from 3D inversion of the data sets and (b) the estimate of the true geometric mean resistivity for the data. Listed are; the number of measurements in the

data sets, the ice thickness (determined from temperature measurements and cores), the number of iterations and the final misfit of the inversion. Both horizontal distances and vertical depths are in metres while dashed lines indicate approximate surfaces of the ice cover. 


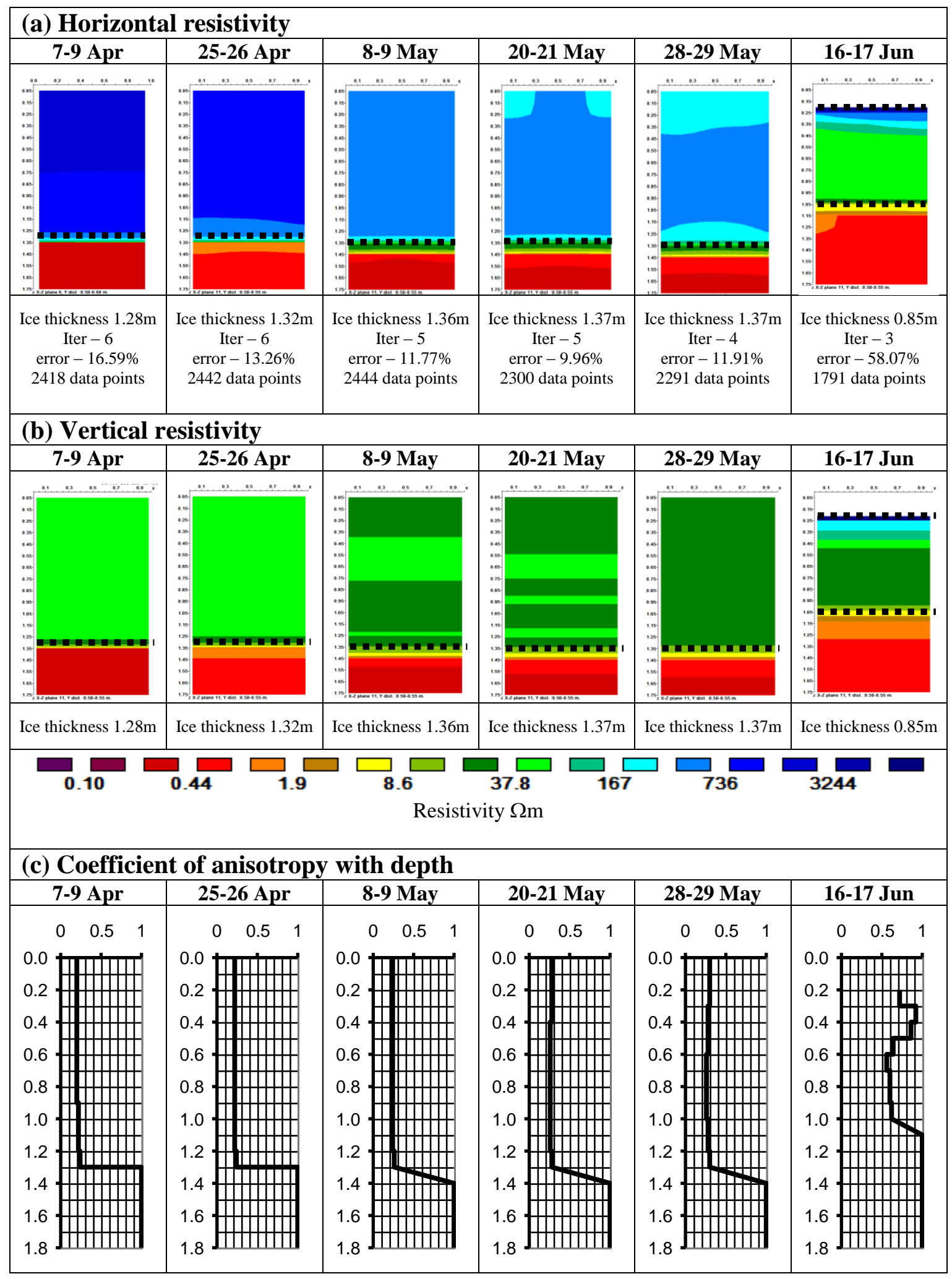

Figure 5.2: Vertical sections through models of the horizontal and vertical resistivity, and anisotropy profiles, 2008 Arctic data. (a) the horizontal resistivity, obtained from 3D inversion of the data and (b) the vertical resistivity, calculated from $\rho_{H}$ and $\rho_{m}$ values. Listed are; the number of measurements in the

data sets, the ice thickness (determined from temperature measurements and cores), the number of iterations and the final misfit of the inversion. Both horizontal distances and vertical depths are in metres

while dashed lines indicate approximate surfaces of the ice cover. (c) shows plots of coefficient of anisotropy vs. depth, obtained while retrieving the true value of $\rho_{m}$. The vertical axis is the depth from the surface in meters and the horizontal axis is the coefficient of anisotropy. 


\begin{tabular}{|c|c|c|c|c|c|}
\hline \multicolumn{6}{|c|}{ (a) Temperature } \\
\hline 7-9 Apr & 25-26 Apr & 8-9 May & 20-21 May & 28-29 May & 16-17 Jun \\
\hline $\begin{array}{ll}-12 & -4\end{array}$ & $\begin{array}{lll}-12 & -4 & 4\end{array}$ & $\begin{array}{ll}-12 & -4\end{array}$ & $-12 \quad-4$ & -12 & -12 \\
\hline 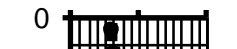 & 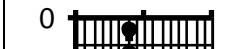 & 0 T & 0 t & 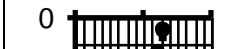 & \\
\hline 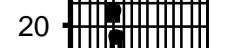 & 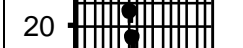 & $20+m$ & 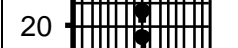 & 20 曲 & 20 . \\
\hline 40 曲的 & 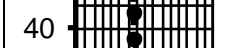 & 40 曲地地皿 & $40 \#$ & $40 \#$ & 40 \\
\hline 60 曲地冊冊冊的 & 60 曲地曲冊曲 & 60 曲地冊 & 曲冊冊 & $60 \#$ & 60 \\
\hline & 80 & $80+m$ & 80 & 80 & 80 \\
\hline $100 \#$ & $100 \mathrm{P}$ & 100 回血 & 100 & $100 \#$ & 100 \\
\hline 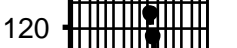 & 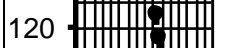 & 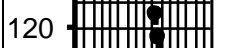 & 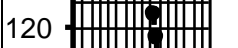 & 120 & 120 . \\
\hline 140 回㘞回地田 & 回回地回 & 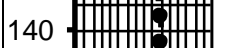 & 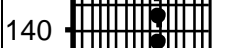 & 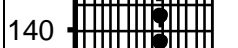 & 140 . \\
\hline 160 曲冊地曲冊曲 & 160 曲冊地冊地 & 160 曲地地冓 & m冊冊地冊 & $160 \#$ & 160 \\
\hline 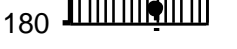 & 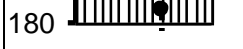 & 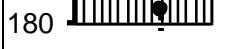 & 180 & $180 \mathrm{Im}$ & 180 \\
\hline \multicolumn{6}{|l|}{ (b) Salinity } \\
\hline $7 \mathrm{Apr}$ & 29 Apr & 29 Apr & 26 May & 26 May & 16Jun \\
\hline 16 & 16 & 16 & 16 & 16 & 16 \\
\hline 0 t & & & (n) & 0 & \\
\hline $20 \#$ & 20 曲 & 20 田地 & $20 \#$ & 20 & 20 . \\
\hline 40 曲 & 40 曲 & $40 \mathrm{~m}$ & $40 \#$ & 40 & 40 \\
\hline 60 曲 & 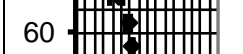 & 60 曲地冊地的 & m-冊冊地 & $60 t$ & 60 \\
\hline $80 \#$ & $80 \#$ & 80 曲地冊冊曲 & 80 & 80 & 80 \\
\hline $100 \#$ & t冓冊囘他 & 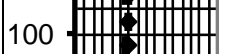 & 100 & 100. & 100. \\
\hline 120 曲 & 120 & 120 & 120 & 120 & 120 \\
\hline 140 曲地曲地 & 140 曲冊地地 & 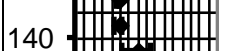 & $140 \#$ & 140 & 140 \\
\hline 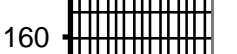 & 160 曲地囘冊曲 & $160 \mathrm{~m}$ & 160 & 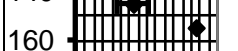 & 160 \\
\hline 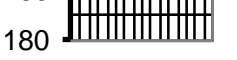 & $180 \mathrm{l}$ & $180 \mathrm{l}$ & $180 \mathrm{~lm}$ & 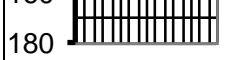 & 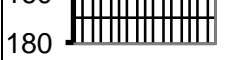 \\
\hline \multicolumn{6}{|c|}{ (c) Brine volume fraction } \\
\hline 7-9 Apr & 25-26 Apr & 8-9 May & 20-21 May & 28-29 May & 16-17 Jun \\
\hline $0 \quad 10$ & 20 & 10 & $0 \quad 10$ & $\begin{array}{lll}0 & 10 & 20\end{array}$ & 10 \\
\hline 0 t & & 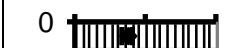 & 0 tII & 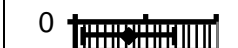 & $0 t$ \\
\hline 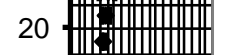 & 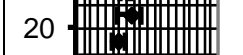 & 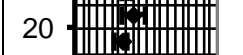 & 20 地苒 & $20 \mathrm{~m}$ & 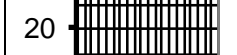 \\
\hline 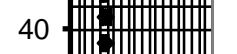 & $40 \mathrm{~m}$ & 40 曲地曲 & 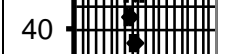 & 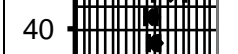 & $40 f$ \\
\hline 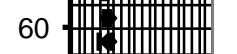 & 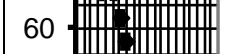 & 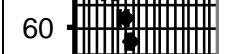 & 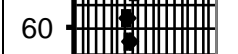 & 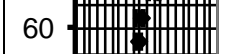 & 帚地 \\
\hline 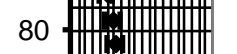 & 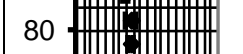 & 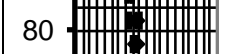 & 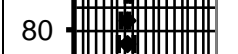 & 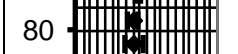 & 80 曲冊地地曲 \\
\hline 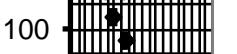 & 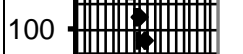 & 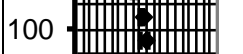 & 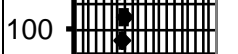 & 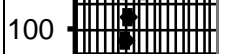 & 100 㗐 \\
\hline $120 \mathrm{fm}$ & 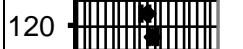 & $120 \mathrm{fm}$ & 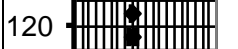 & $120 \mathrm{mb}$ & $120 \mathrm{fm}$ \\
\hline $140 \mathrm{~m}$ & 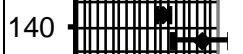 & 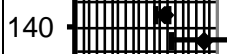 & 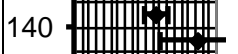 & 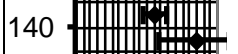 & 140 \\
\hline 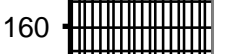 & $160 \mathrm{fm}$ & 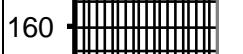 & $160 \mathrm{~m}$ & 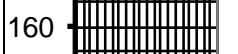 & 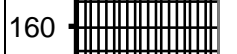 \\
\hline 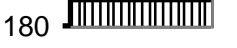 & 180 & 180 & 180 & $180 \mathrm{~d}$ & $180 \mathrm{~d}$ \\
\hline
\end{tabular}

Figure 5.3: Temperature, salinity and brine volume fraction profiles, 2008 Arctic measurements. (a) smoothed averaged temperatures with depth - obtained from thermistor string measurements at the UAF mass balance site, (b) ice salinity with depth - obtained from measurements made on ice cores from around the UAF mass balance site, and (c) brine volume fraction calculated from salinity and temperature, as in Cox and Weeks (1983), and plotted against depth. The vertical axis on all plots is depth from surface in centimetres. The horizontal axes are (a) temperature in ${ }^{\circ} \mathrm{C}$, (b) salinity in psu and (c) brine volume fraction as a percentage. 
(a) Horizontal resistivities

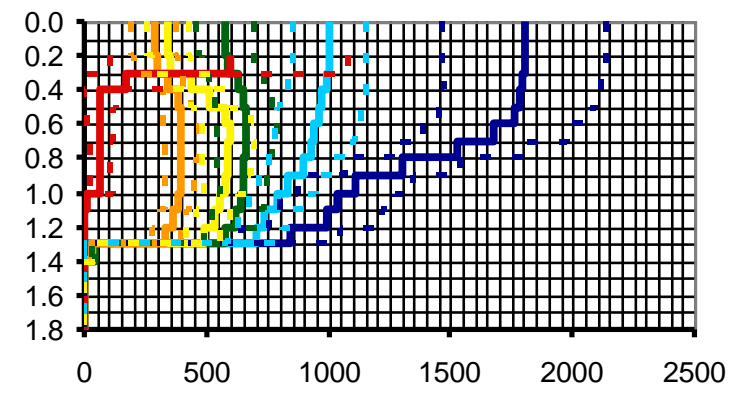

(b) Geometric mean resistivities

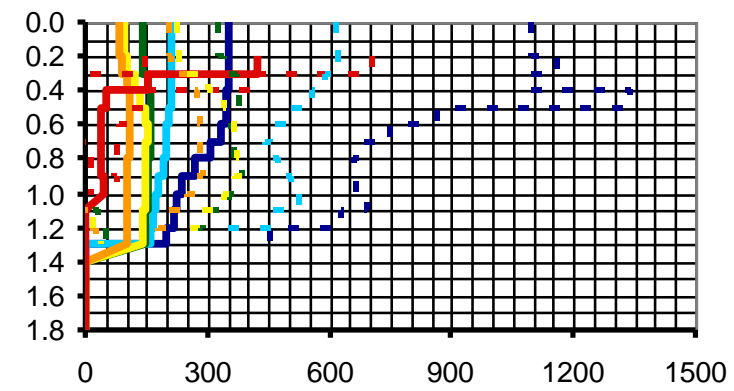

(c) Vertical resistivities

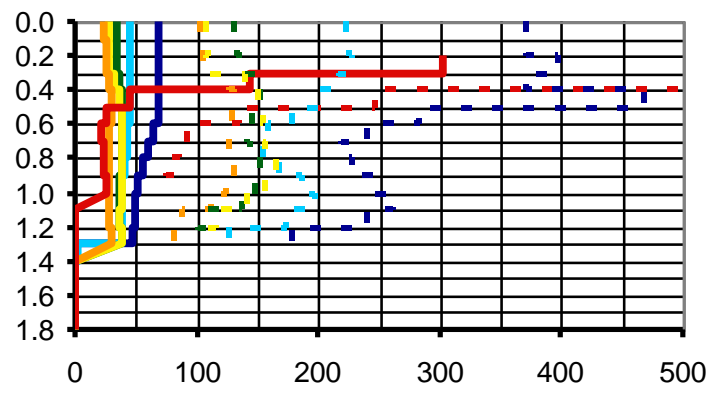

(d) Coefficient of anisotropy

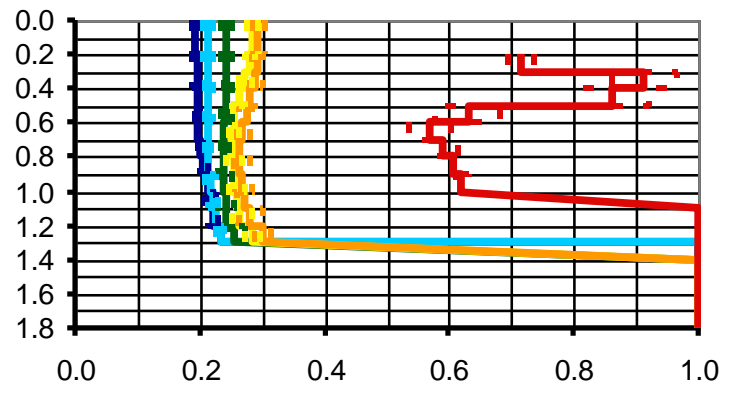

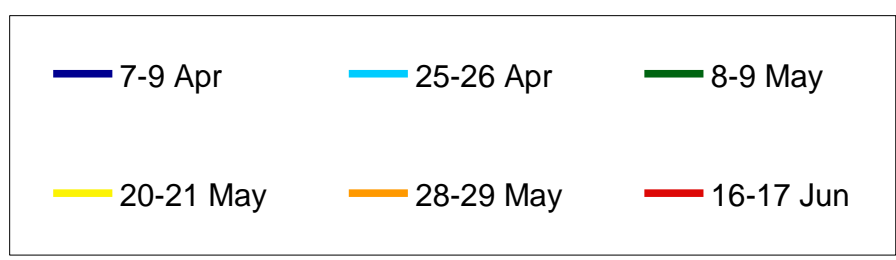

Figure 5.4: Line plots of average 2008 data values with depth for (a) the horizontal resistivity, (b) the geometric mean resistivity, (c) the vertical resistivity and (d) the coefficient of anisotropy of all six data sets. The vertical axes are depth from surface in meters and the horizontal axes are resistivity in ohm meters except (d) where the horizontal axis is the coefficient of anisotropy. Dashed lines indicate estimates of the uncertainties in the values.

It should be noted, that the inversion modelling technique used (discussed in Chapter 4) does not necessarily produce unique models. Thus, as with all geophysical modelling, one must attempt to obtain physically realistic results and apply constraints from known parameters.

The datasets for determination of $\rho_{H}$ contained approximately 1800 to 2400 separate measurements (Figure 5.2a). Those for $\rho_{m}$ were smaller and contained between 320 and 600 separate measurements (Figure 5.1a). The ice thickness at the time of each set of measurements was determined from ice temperature measurements and cores (Chapter 3). Over the measurement period the ice thickness, measured by the UAF mass balance site, increased from $1.28 \mathrm{~m}$ to $1.38 \mathrm{~m}$ before decreasing, during significant melt, to be $0.83 \mathrm{~m}$ at the time of the last measurement. During the inversion process the presence of 
sea water beneath the ice was incorporated by using an initial model where, below the depth of the measured ice thickness, the resistivity was set to approximate that of seawater $(0.4 \Omega \mathrm{m})$. The degree to which resistivity values in this 'fixed' region could change was controlled during the inversions (as mentioned in Chapter 4).

The 3D inversions for $\rho_{H}$ typically took 4-6 iterations and gave final misfits of between $10 \%$ and $17 \%$ (Figure 5.2a), while those for the geometric mean resistivity took 5-6 iterations and gave final misfits of $2 \%-11 \%$ (Figure 5.1a). However, the true $\rho_{m}$ obtained numerically from the inversion resistivities $\left(\rho_{H}\right.$ and $\rho_{m}^{M}$ ), has much larger uncertainties (as can be seen in Figure 5.4b). Much of the misfit in the $\rho_{H}$ inversions is probably due to the ice-water interface. In this region steep gradients in resistivity occur, with a rapid change in resistivity from several hundred ohm meters to a value less than $1 \Omega \mathrm{m}$, which are difficult to model accurately with a numerical grid of finite size. Due to the lower value of $\rho_{m}$ there is a smaller decrease across this same region, which can be much better reproduced, probably explaining the lower misfits observed in the $\rho_{m}$ inversions. The inversion models for both $\rho_{H}$ and $\rho_{m}$ on the 16-17 June have high misfits, possibly due to the large range of resistivities observed in these models.

Assumptions made in the theory (see Chapter 2) mean it is worth considering the magnitude of resistivity changes that are likely to occur as the microstructure of sea ice changes due to expansion and connection of brine inclusions. A simple means of doing this is to consider the Hashin - Shtrikman limits (Hashin and Shtrikman, 1962) for a two phase mixture. The limits were established for the effective magnetic permeability of a medium. However, the results are also valid for the dielectric constant, electrical conductivity, heat conductivity and diffusivity.

Consider a two-phase mixture in which isolated spheres of resistivity $\rho_{2}$ are surrounded by a connected matrix of resistivity $\rho_{1}$. To obtain a mixture representative of sea ice values for the resistivity of the brine and ice are set at $0.2658 \Omega \mathrm{m}$ (average brine resistivity calculated from the measured data) and $10^{7} \Omega \mathrm{m}$ (the DC resistivity of pure ice as noted by Mathews and Clark (1963)) respectively. In this case it can be shown (Figure 5.5) that for brine volume fractions of between about 5\% and 95\%, the decrease in bulk resistivity between situations when brine pores are isolated ( $\rho_{2}$ equals the resistivity of brine and $\rho_{l}$ equals the resistivity of ice) and when a connected network of 
brine exists ( $\rho_{2}$ equals the resistivity of ice and $\rho_{l}$ equals the resistivity of brine) is 5-6 orders of magnitude.

However, the conductivity of the solid ice matrix is not well constrained and a range of conductivities should be considered. Even if the ice has a low resistivity of $1000 \Omega \mathrm{m}$ (extremely unlikely as most salt is rejected from the ice matrix and some of the measured resistivities of the sea ice are higher than this value) then there is still a difference of 1-2 orders of magnitude between the cases of isolated and connected brine. Given this order of magnitude for the likely changes in resistivity, it is clear that, in terms of tracking the changes that occur in the microstructure of sea ice it is critical to be able to resolve between resistivity values of, for example, $20 \Omega \mathrm{m}$ and $200 \Omega \mathrm{m}$, however, it is not necessary to be able to accurately discriminate between values of, say, $200 \Omega m$ and $400 \Omega m$.

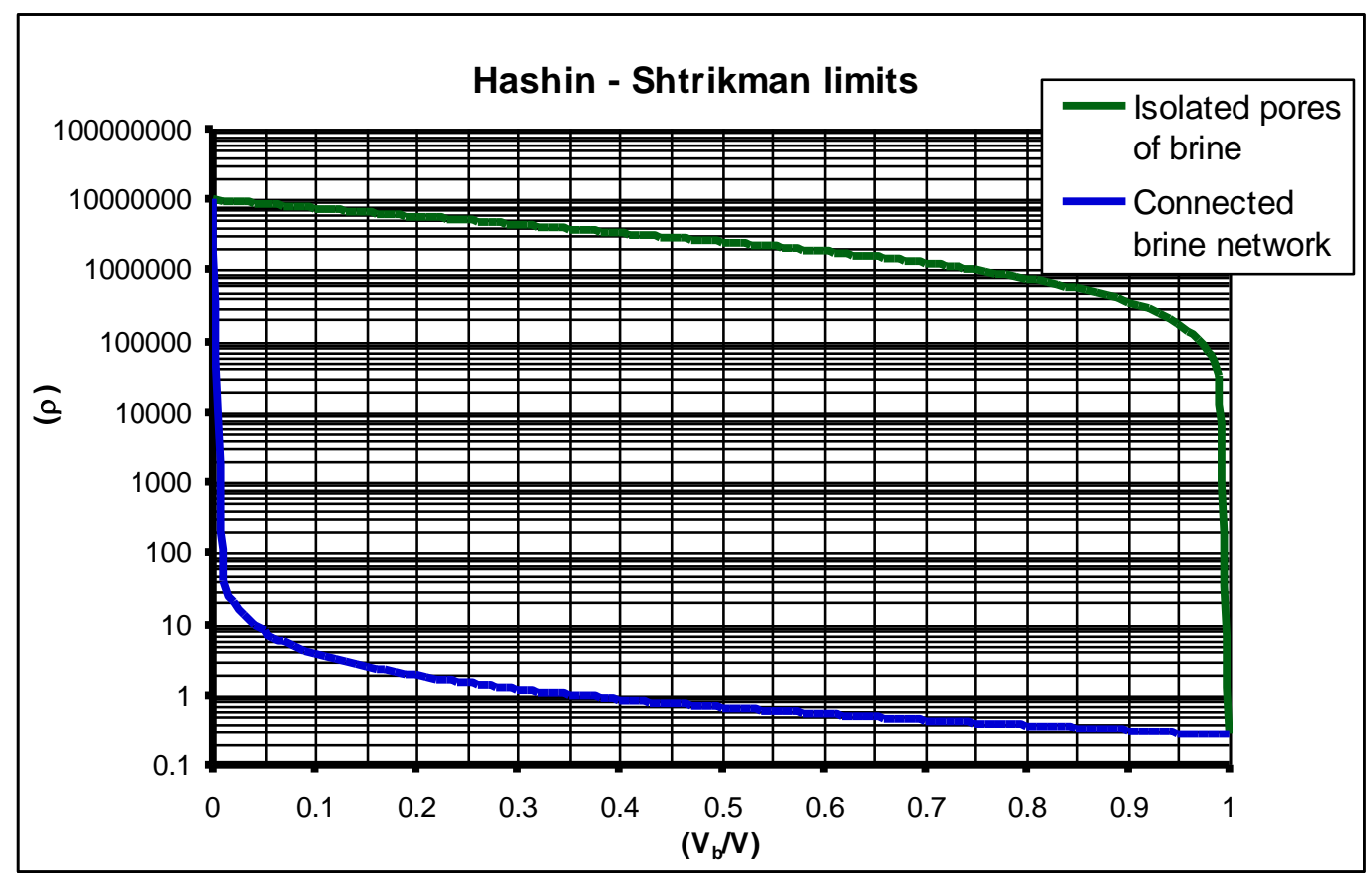

Figure 5.5: Plot of resistivity vs. brine volume fraction displaying the behaviour of the Hashin Shtrikman limits for a two-phase mixture of brine $(\rho \sim 0.2658 \Omega \mathrm{m})$ and ice $(\rho \sim 10000000 \Omega \mathrm{m})$, considering two cases - isolated pores of brine and a connected brine network.

The inversions use a grid with horizontal spacing of $0.05 \mathrm{~m}$ in both $x$ and $y$ directions, and a vertical spacing of $0.1 \mathrm{~m}$. There is one exception, on the 7-9 Apr the horizontal grid is set to $0.1 \mathrm{~m}$ (see section 4.2.1). The vertical discretisation of the models leads to sharp gradients in the recovered values of resistivity around the seawater/ice interface. 
Figure 5.2a it can be seen that in general the horizontal component of the bulk resistivity of the sea ice displays high values of between $300 \Omega \mathrm{m}$ to $1800 \Omega \mathrm{m}$. These values are within the range of $200 \Omega \mathrm{m}$ to $5000 \Omega \mathrm{m}$ recorded by Ingham et al. (2008) (see Figure 5.8 below) using cross-borehole measurements, with just two boreholes rather than four. Thyssen et al. (1974) and Timco (1979) commented on measurements made using an array of electrodes located vertically on the wall of an ice pit, to obtain the horizontal resistivity of sea ice. They quoted values of $221 \Omega \mathrm{m}$ and $129 \Omega \mathrm{m}$ to $298 \Omega \mathrm{m}$ respectively. Although these are rather low in comparison to our results from early in the spring they are within the range of resistivities ( $50 \Omega \mathrm{m}$ to $400 \Omega \mathrm{m})$ observed between late May and mid June, by these dates the ice has warmed somewhat giving these lower resistivities.

The general decrease observed in our horizontal resistivities as the temperature of the ice increases from early April to late May (Figure 5.2a and Figure 5.4a) is likely to indicate a gradual change in the microstructure of the ice as connectivity of the brine pockets increases in the horizontal direction. Increased connectivity between brine pockets is also supported by an increase in brine volume fraction (Figure 5.3c) over the measurement period. The low horizontal resistivities, warm temperatures and high brine volume fractions observed on the 16-17 June suggest significant horizontal connectivity of the brine inclusions by this stage.

Initially the horizontal resistivity structure gives high surface values which decrease gradually with depth until a sharp drop at the ice/seawater interface. However, further into the melt season a region of low resistivity appears at the surface of the ice (Figure 5.2a and Figure 5.4a). This was also observed by Ingham et al. (2008), as shown in Figure 5.8below. These low resistivities, matched with high temperatures, indicates that surface warming has led to a significant increase in connectivity of the brine structure at shallow depth. It should also be noted that by the 16-17 June there was significant surface melt and the top two - three electrodes of the strings were protruding from the ice surface. Furthermore, the higher resistivities seen at the surface of this model would seem to indicate a region flushed of brine by increasing melt water.

Compared with the $\rho_{H}$ inversions, there is much less variation in the derived value of $\rho_{m}^{M}$ (Figure 5.1a) with the inversions of the geometric mean resistivity data producing values of the order of $10 \Omega m-20 \Omega m$. However, the estimate of the true geometric mean 
resistivity retrieved through numerical modelling (Figure 5.1b) ranges from $100 \Omega \mathrm{m}$ to $350 \Omega \mathrm{m}$. The values obtained for the 16-17 June are significantly lower than the rest of the data, with values of $\sim 40 \Omega \mathrm{m}$.

In general these values are slightly higher than geometric mean resistivities obtained from previous studies which use surface soundings. For first year sea ice near Pond Inlet, N. W. T. Thyssen et al. (1974) obtain resistivities of between 30 and $176 \mathrm{ohm}$ meters while Timco (1979) gave values of $76 \Omega \mathrm{m}$ to $176 \Omega \mathrm{m}$. On Antarctic sea ice Buckley et al. (1986) stated values of $50 \Omega \mathrm{m}$ to $200 \Omega \mathrm{m}$. In our Barrow study, a number of surface resistivity soundings carried out adjacent to the location of the borehole measurements yielded geometric mean resistivities in the range of $50 \Omega \mathrm{m}$ to $100 \Omega \mathrm{m}$, These values are about a factor of three smaller than the geometric mean retrieved from numerical modelling and around five times larger than the values obtained directly from $3 \mathrm{D}$ inversion of the cross-borehole measurements.

This lends some support to the suggestion that the cumulative effect of uncertainties and approximations in the retrieved $\rho_{m}$ may mean that the values derived from the borehole measurements should be regarded as an estimate correct to within a factor of $3-4$. However, various authors have previously noted the non-uniqueness in the interpretation of Wenner soundings, particularly the distortions in derived depth variations that occur in interpretation due to the anisotropic nature of the resistivity. It could therefore be argued that the estimates of $\rho_{m}$ derived from the cross-borehole results are in fact more reliable. Nevertheless, in the context of mapping the variation of resistivity structure with time over a series of measurement sequences, such an unresolved ambiguity (of a factor of $3-4$ ) may not necessarily be crucial, as changes in structure are likely to produce at least a change of magnitude in the resistivities.

The vertical resistivity structure (Figure 5.2b), calculated from $\rho_{H}$ and $\rho_{m}$, gives low resistivities of the order of $20 \Omega \mathrm{m}$ to $70 \Omega \mathrm{m}$. This suggests a significant degree of vertical connection between brine inclusions even at the start of the measurement period when the ice was still relatively cool. From ice pit measurements Timco (1979) obtained values of $45 \Omega \mathrm{m}$ to $105 \Omega \mathrm{m}$ for the vertical resistivity of first year sea ice, comparable to the results presented here. However, Thyssen et al. (1974) also used ice pit measurements and obtained a lower vertical resistivity of $16 \Omega \mathrm{m}$. 
Through the measurement period the vertical resistivity decreases, but not as significantly as does the horizontal resistivity. As would be expected, from the trends of the horizontal and vertical resistivities, the geometric mean resistivity also shows a decreasing trend. Though we observe this increase in conductivity (or decrease in resistivity) over the measurement period i.e. an increase with increasing temperature and brine volume fraction, we do not note a strong linear relationship with brine volume or an exponential relationship with temperature as was observed by Morey et al. (1984) for DC conductivity. Plots of conductivity versus (a) temperature and (b) brine volume fraction can be seen for both the vertical and horizontal conductivities in Figure 5.6 below.

(a)

Conductivity vs. temperature

Horizontal conductivity

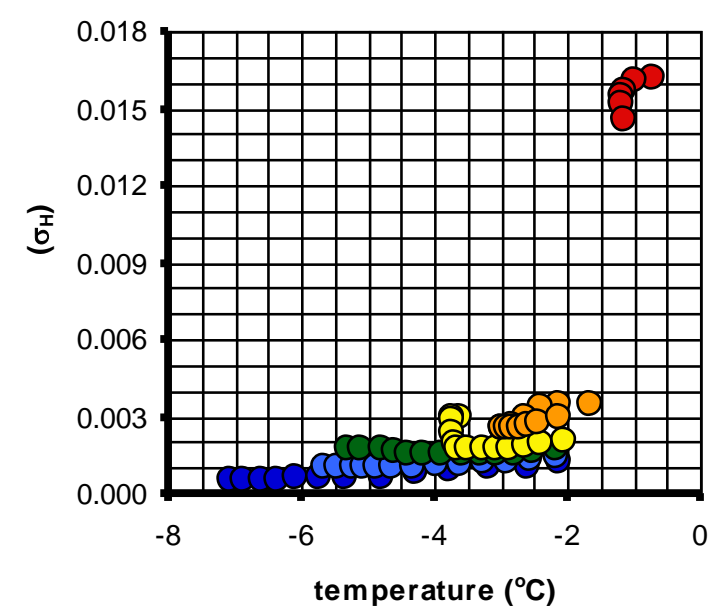

Vertical conductivity

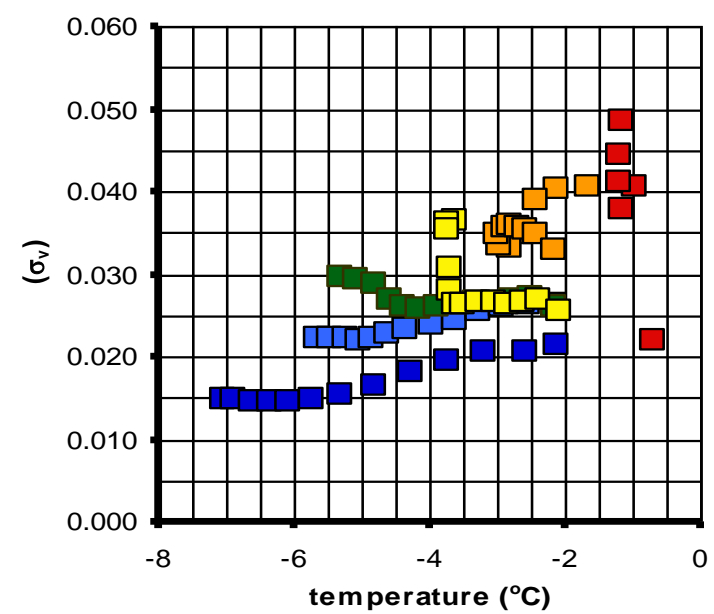

(b) Conductivity vs. brine volume fraction
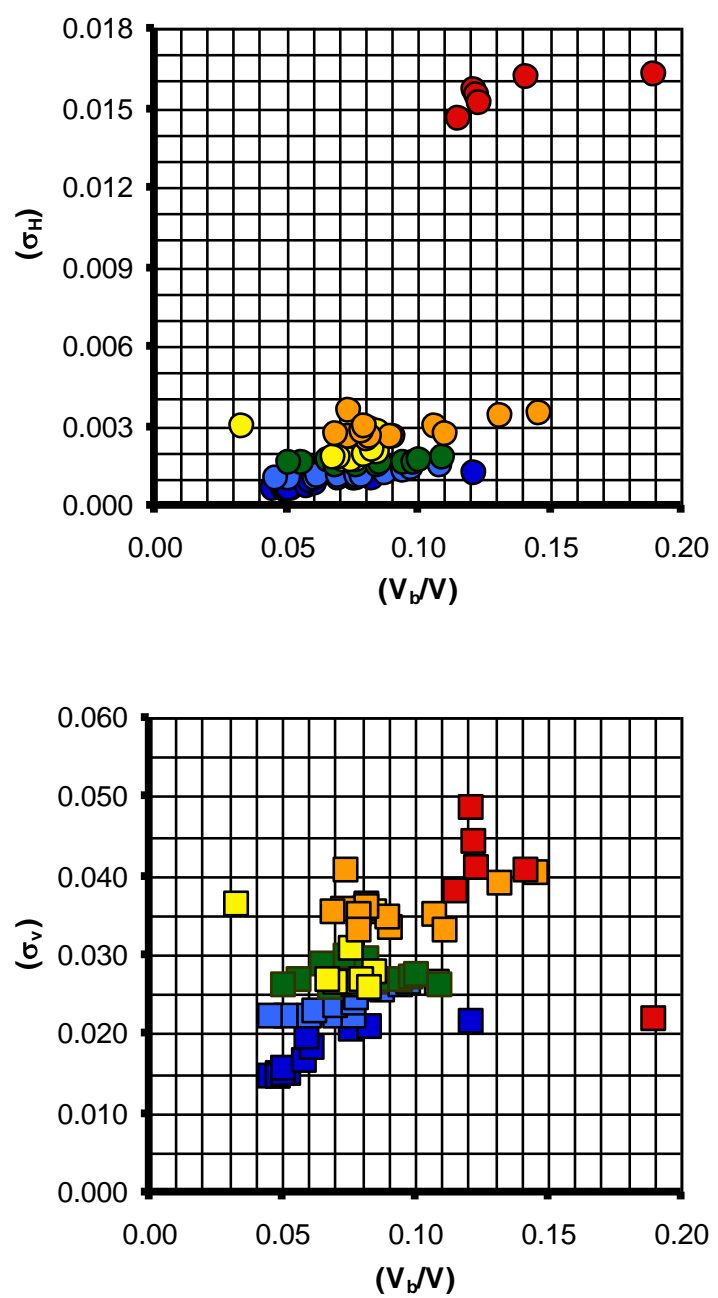

\begin{tabular}{|lll|}
\hline$\square 7-9$ Apr & $\square 25-26$ Apr & $\square 8-9$ May \\
$\square 20-21$ May & $\square 28-29$ May & $\square 16-17$ Jun \\
\hline
\end{tabular}

Figure 5.6: Plots of the relationships between conductivity and (a) temperature, (b) brine volume fraction, during the measurement period in the Arctic. 
For most data sets obtained over the measurement period the anisotropy coefficient (Figure 5.2c) is found to be relatively constant through the entire thickness of the ice. Though there is a slight increase with depth, we do not observe any significant trend of increasing $\lambda$ with depth, as was indicated by Morey et al. (1984) for sea ice with a preferential horizontal alignment of c-axes. Values for the anisotropy coefficient $(\lambda)$ range from approximately 0.2 in early April to around 0.3 in late May, indicating a change in the structure of the ice as $\rho_{H}$ and $\rho_{V}$ become closer in value. The derived values of $\lambda$ are slightly less than values found, for example, by Timco (1979) (0.59) and Thyssen et al. (1974) (0.26 to 1) at Pond Inlet, N.W.T. and Buckley et al. (1986) in the Antarctic ( 0.5), but are not unreasonable for sea ice formed under the quiescent conditions which tend to exist at Barrow.

Analysis of ice cores by UAF (Eicken, H., pers. comm., 2010) indicate that the initial granular or frazil growth of the ice cover is present to depths of $40 \mathrm{~cm}$. However, there is no indication of this structure in either the vertical or horizontal resistivity profiles or the variation of the coefficient of anisotropy with depth. In the upper regions of the sea ice the number of measurements that can be made is limited, simply due to the electrodes being constrained by the surface of the ice. Having fewer data will decrease the resolution of the resistivity model and may explain why we do not observe the surface frazil ice. Alternatively, it is possible that the change in the brine structure is not significant enough to be observed in the resistivity structure, i.e. even though the ice grains have different orientations in the random frazil ice and the vertically aligned columnar ice the degree and direction of connectivity between individual brine inclusions maybe similar.

The model sections obtained from RES3DINV, during inversion of the horizontal resistivity data, seem to show the positions of the electrode strings, as indicated by anomalous resistivity values (Figure 5.7). A halo of anomalous resistivity structure around electrode strings was also observed by Ingham et al. (2008). This clearly shows why it is essential to carry out cross-borehole measurements, so the results are obtained from undisturbed sea ice. Where average values are used in calculations, data within $0.2 \mathrm{~m}$ of the corners of the measurement area, defined by the electrode strings, are ignored. By not considering the data from within these areas (as indicated by the shading in Figure 5.7 below) the average resistivity will be of the sea ice and not due to the presence of the boreholes and electrode strings. 

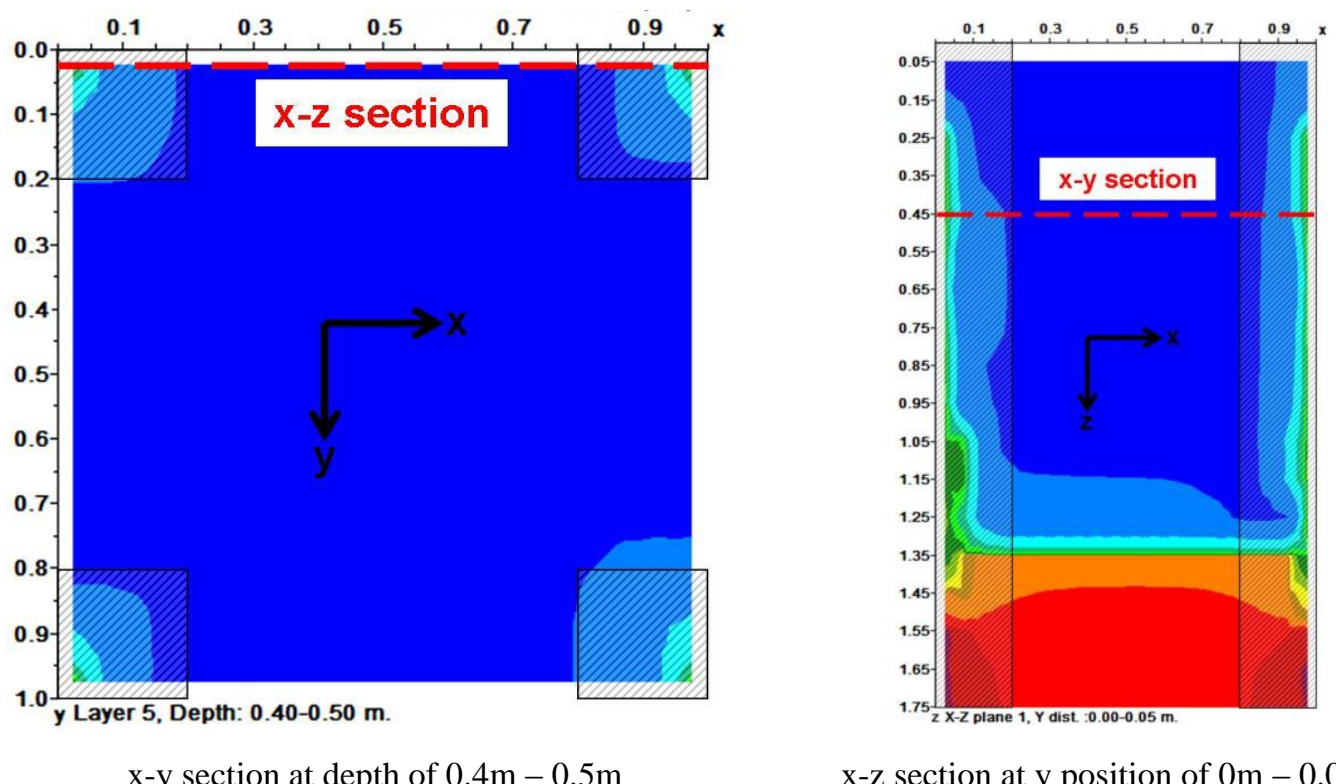

$\mathrm{x}-\mathrm{y}$ section at depth of $0.4 \mathrm{~m}-0.5 \mathrm{~m}$

$\mathrm{x}-\mathrm{z}$ section at $\mathrm{y}$ position of $0 \mathrm{~m}-0.05 \mathrm{~m}$

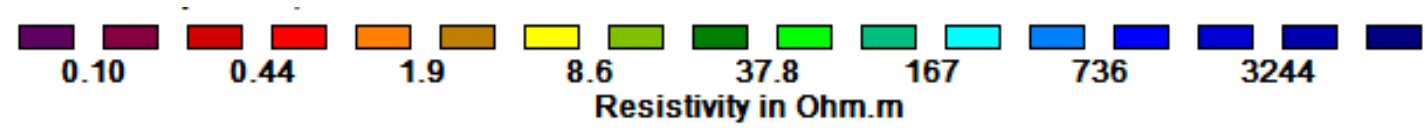

Figure 5.7: $x-y$ and $x-z$ sections of the horizontal resistivity model - displaying the anomalous halos around the electrode strings. The $x-y$ section is at a depth of $z=0.4 \mathrm{~m}-0.5 \mathrm{~m}$ and the $\mathrm{x}-\mathrm{z}$ section is a $\mathrm{y}$ position of $0 \mathrm{~m}-0.05 \mathrm{~m}$, as shown in the diagram.

\subsubsection{6 measurements - Ingham et al. (2008)}

In 2006 Ingham et al. (2008) made three separate cross-borehole resistivity measurements as the ice warmed over the period April - June. Their measurement site was located in first year sea ice offshore from Pt. Barrow, Alaska $\left(71^{\circ} 22^{\prime} 03^{\prime \prime} \mathrm{N}, 156^{\circ}\right.$ 31' 03 " W). Measurements of the horizontal resistivity structure of the ice were made using appropriate electrode combinations between two vertical strings of electrodes, installed in boreholes drilled $1 \mathrm{~m}$ apart. The first series of measurements was made between 22-25 April at which time the ice thickness was $1.38 \mathrm{~m}$, the second on 11 May (ice thickness $1.46 \mathrm{~m}$ ), and the final series on 8 June (ice thickness $1.54 \mathrm{~m}$ ). Vertical sections through the models obtained from 3D inversion of this data are shown in Figure 5.8 below, along with temperature, salinity and brine volume fraction profiles.

As observed in our Arctic data set, the horizontal resistivity obtained by Ingham et al. (2008) decreases over the measurement period as the temperature increases. Furthermore, as mentioned earlier, the resistivities are similar to those obtained in our 
2008 study and a region of low surface resistivity appears in later measurement series as it does in our data. Comparing the models of the horizontal resistivity obtained from the Ingham et al. (2008) study and our 2008 Arctic measurements; it can be seen that data obtained on the 7-9 and 25-26 Apr 2008 is similar to the 22-25 Apr 2006 data, the 8-9, 20-21 and 28-29 May 2008 is comparable to the 11 May 2006, and the 16-17 Jun 2008 data is closest to that obtained on the 8 Jun 2006. 

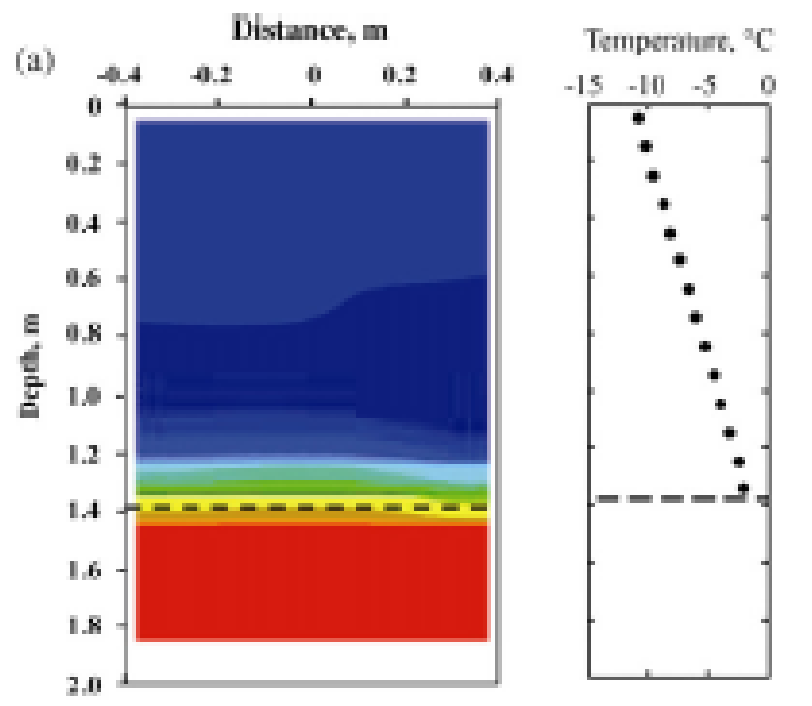

Salinity. ppt

Brine volume, $\%$
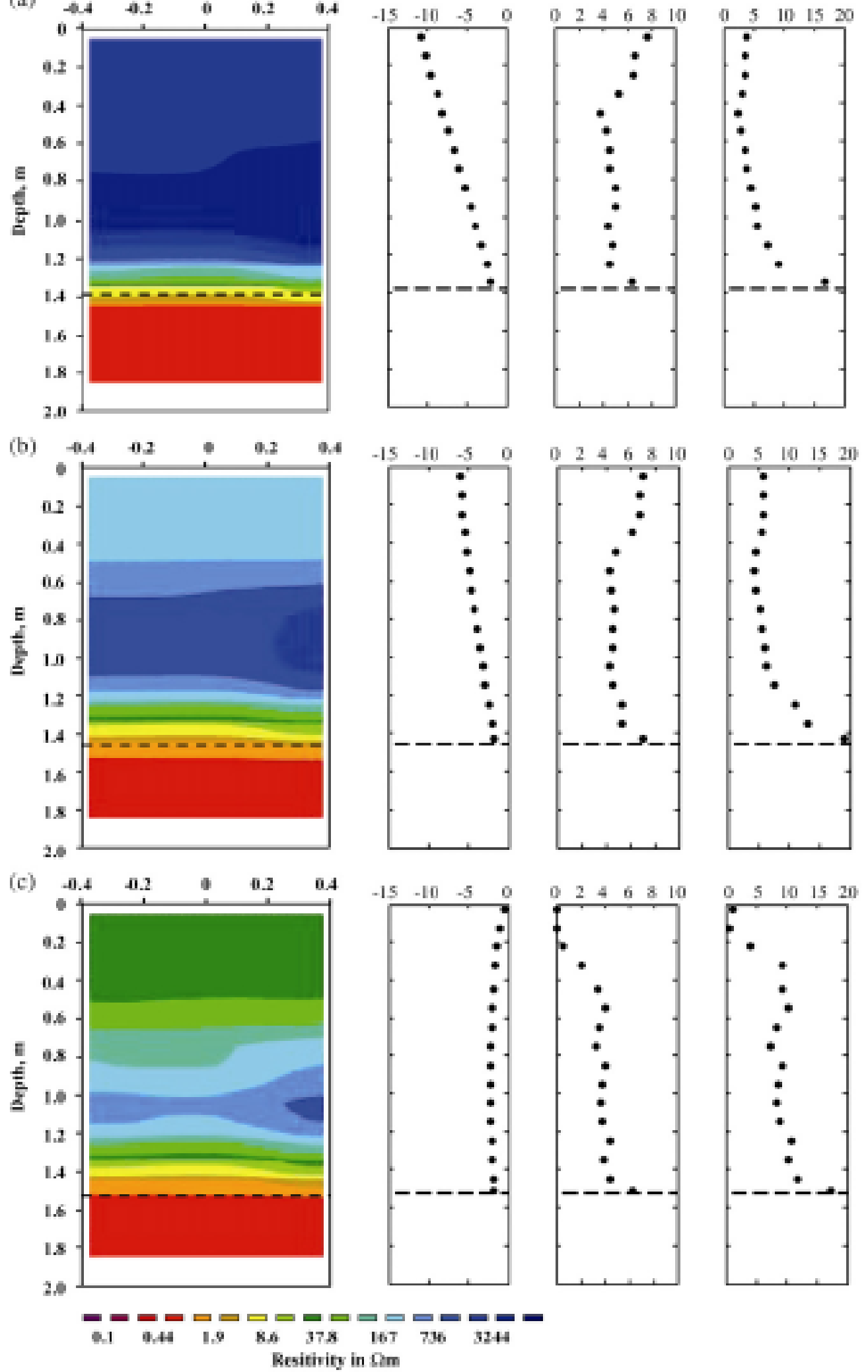

Figure 5.8: Vertical sections through the 3D horizontal resistivity models, as obtained from Fig. 5. in Ingham et al. (2008). (a) 22-25 April 2006, (b) 11 May 2006 and (c) 8 June 2006. Also shown are profiles of sea ice temperature (from an in-situ thermistor string), salinity (from cores) and calculated brine volume fraction. The dashed line marks the measured ice/water interface. 


\subsection{McMurdo Sound, Antarctica - 2009}

A further four measurement sets were obtained over a two week period in November 2009, also in first year sea ice but this time off the coast of Ross Island, Antarctica (as discussed in Chapter 3). This section presents vertical sections of the resistivity models obtained from these data.

Geometric mean resistivities are displayed in Figure 5.9 both for (a) sections through the models obtained from inversion of the data (an underestimation of the resistivity value) and (b) the retrieved estimation of the true value (by a process summarised above in section 5.1 and detailed in section 2.3.2.2).

Figure 5.10 shows (a) sections of the horizontal resistivity obtained from 3D inversion of the data, along with (b) the vertical resistivity structure - calculated from the horizontal and geometric mean resistivities and (c) the coefficient of anisotropy $(\lambda)$ determined while retrieving the true $\rho_{\mathrm{m}}$.

Figure 5.11a shows plots of the depth dependence of the temperatures, measured as part of Antarctica New Zealand science event K131 using thermistor strings. Salinity profiles, obtained as part of K131 via standard measurements on sea ice cores, are shown in Figure 5.11b. Two salinity profiles were obtained during the resistivity measurement period and these are combined in this plot. Figure $5.11 \mathrm{c}$ displays the brine volume fraction, calculated, as previously, from standard relations given in Cox and Weeks (1983) using the combined salinity profile in (b) and the temperatures measured on the salinity cores.

For each of the components of the bulk resistivity line plots of the average values with depth are shown in Figure 5.12, along with a plot of the change in the profile of the coefficient of anisotropy over this measurement period. The standard deviations associated with the average values of $\rho_{H}$ and $\rho_{m}^{M}$ in each layer and the absolute misfit of the models to the observed data provide uncertainties in $\rho_{H}, \rho_{m}, \rho_{V}$ and $\lambda$. 


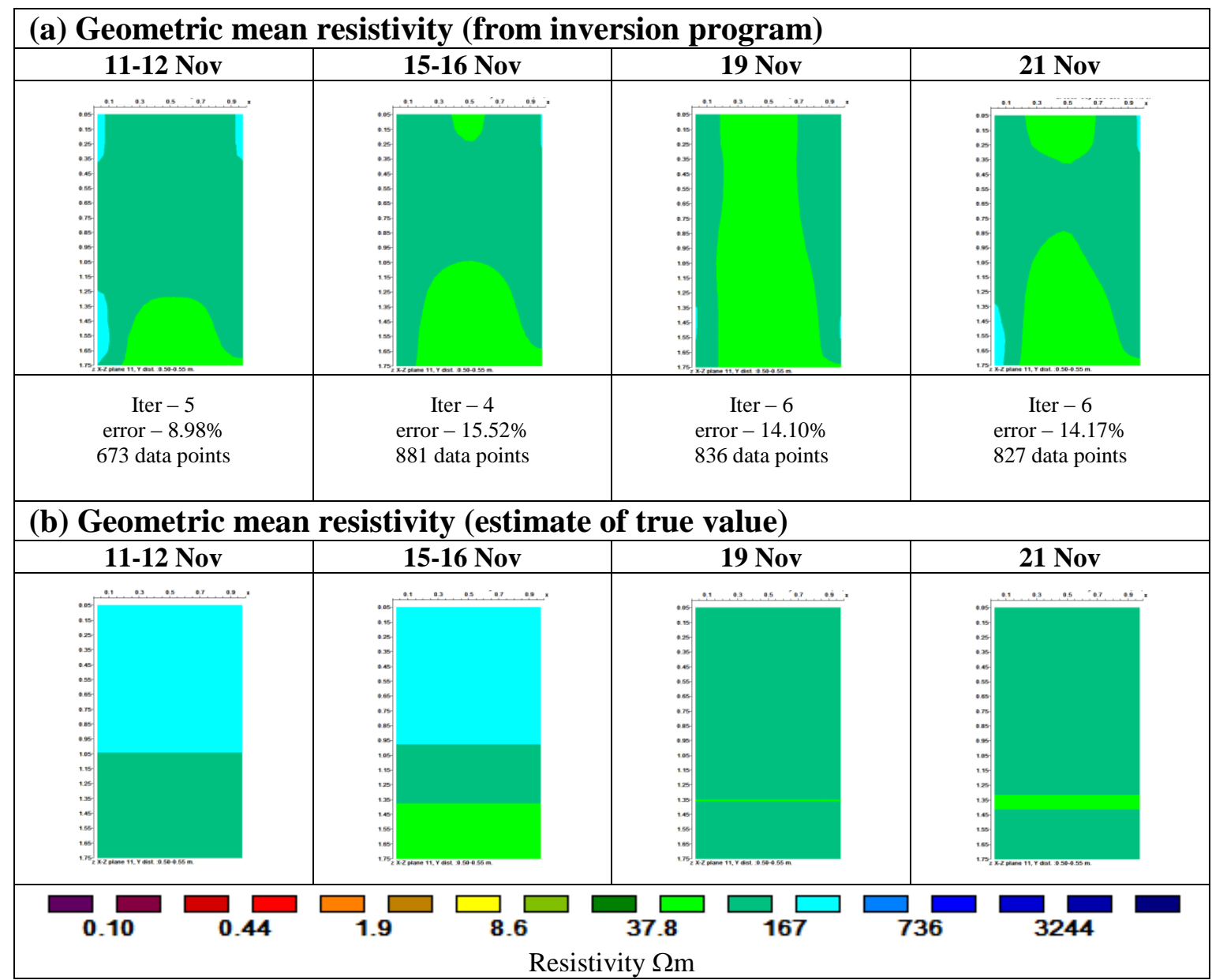

Figure 5.9: Vertical sections through models of the geometric mean resistivity, 2009 Antarctic measurements. (a) the geometric mean resistivity obtained from 3D inversion of the data sets and (b) the estimate of the true geometric mean resistivity for the data. Listed are; the number of measurements in the data sets, the number of iterations and the final misfit of the inversion. The ice thickness at $\sim 2.41 \mathrm{~m}$ is not shown. Both horizontal distances and vertical depths are in metres. 


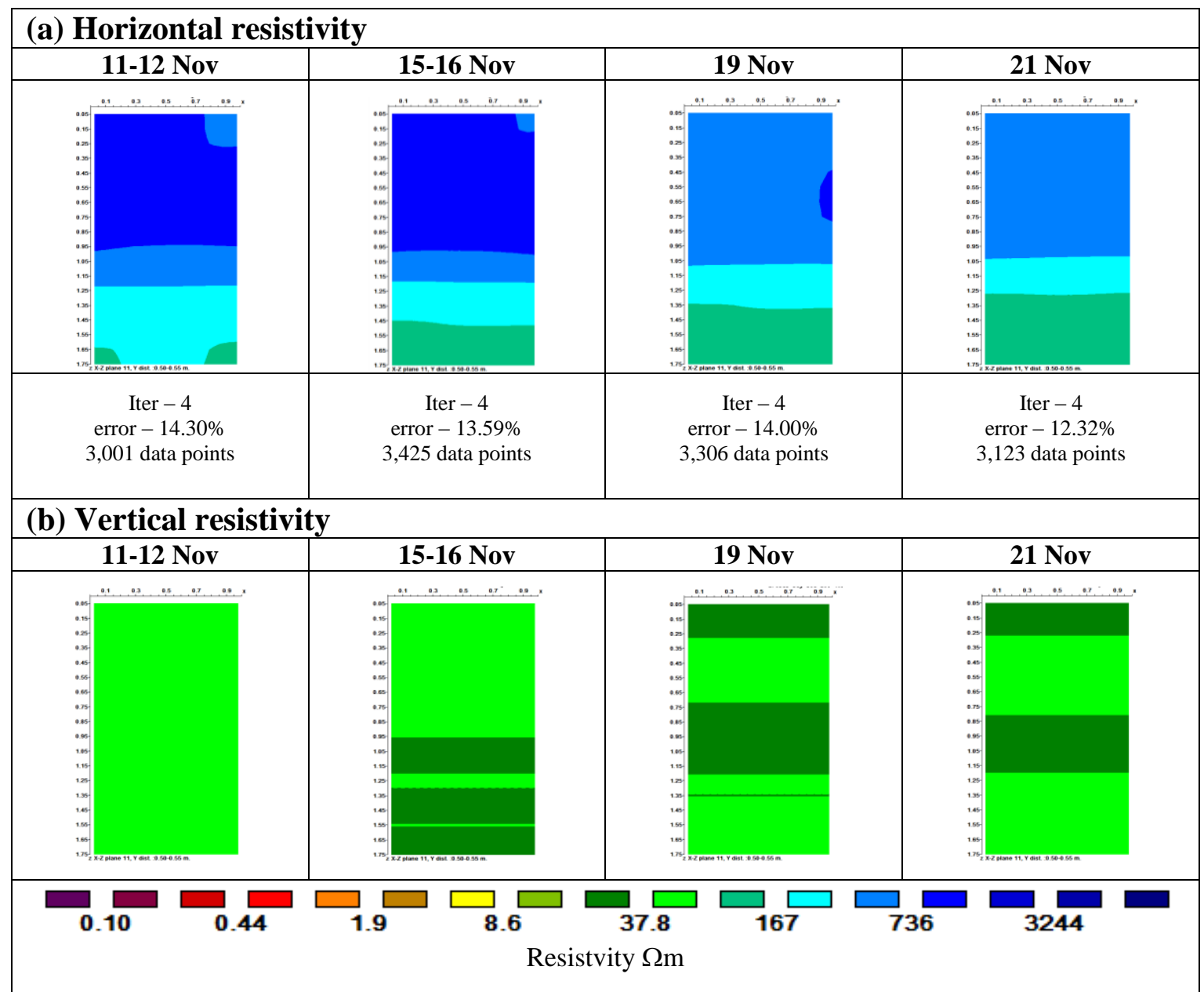

(c) Coefficient of anisotropy with depth

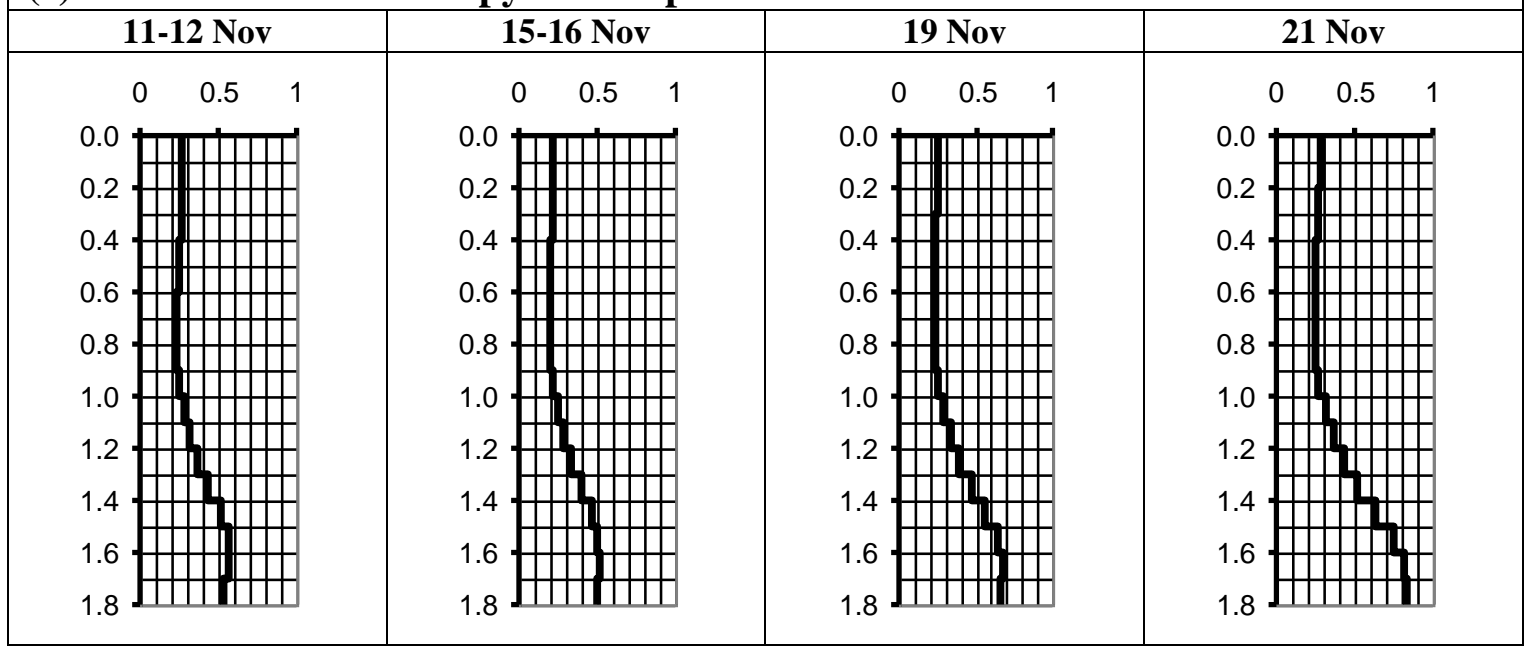

Figure 5.10: Vertical sections through models of the horizontal and vertical resistivity, and anisotropy profiles, 2009 Antarctic data. (a) the horizontal resistivity, obtained from 3D inversion of the data sets and (b) the vertical resistivity, calculated from $\rho_{H}$ and $\rho_{m}$ values. Listed are; the number of measurements in the data sets, the number of iterations and the final misfit of the inversion. The ice thickness at $\sim 2.41 \mathrm{~m}$ is not shown. Both horizontal distances and vertical depths are in metres. (c) shows plots of coefficient of anisotropy vs. depth, obtained while retrieving the true value of $\rho_{m}$. The vertical axis is the depth from the surface in meters and the horizontal axis is the coefficient of anisotropy. 


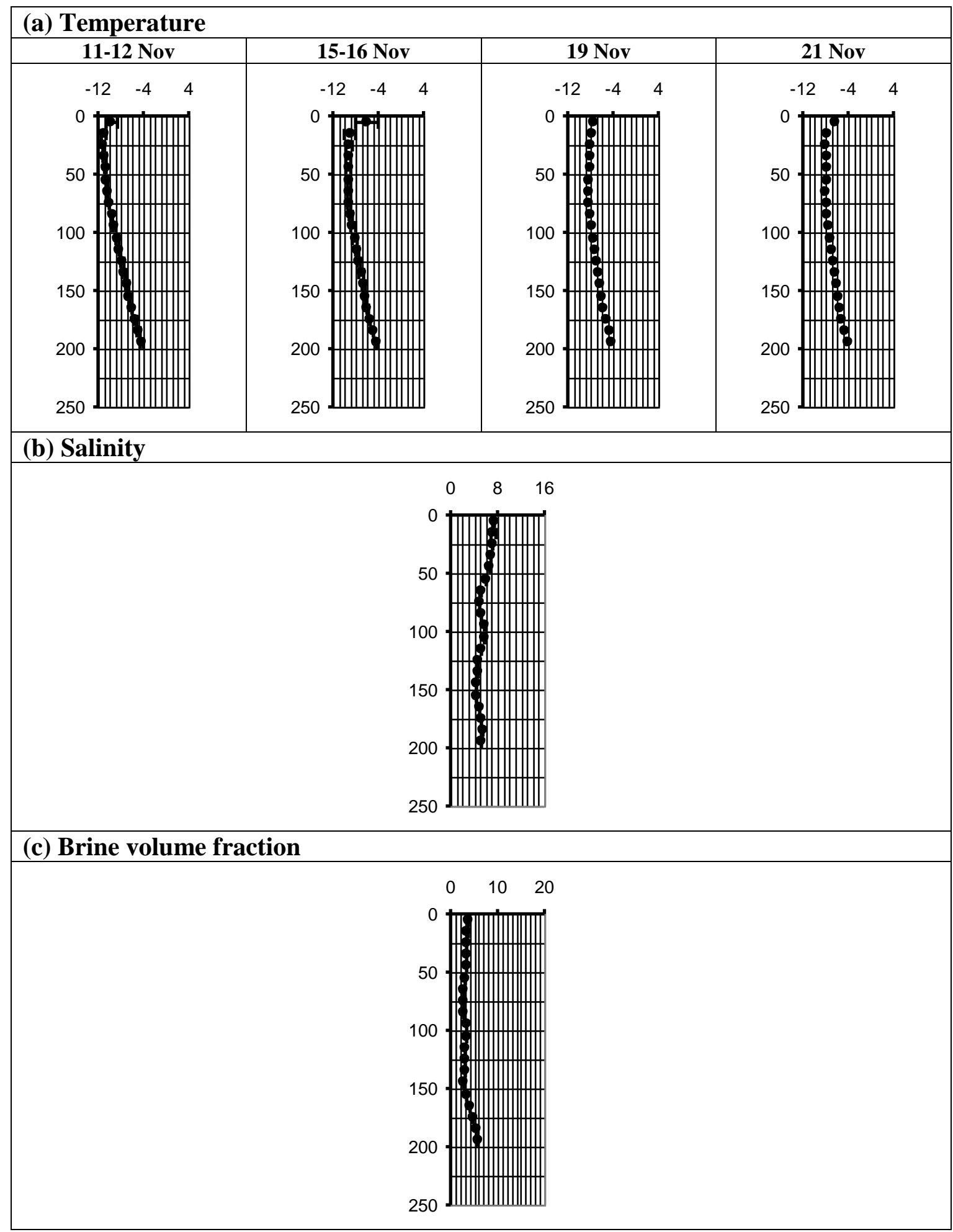

Figure 5.11: Temperature, salinity and brine volume fraction profiles, 2009 Antarctic measurements. (a) smoothed averaged temperatures with depth - obtained from thermistor string measurements made as part of Antarctica New Zealand science event K131, (b) ice salinity with depth - obtained from measurements made on ice core as part of K131, and (c) brine volume fraction calculated from salinity and temperature, as in Cox and Weeks (1983), and plotted against depth. The vertical axis on all plots is depth from surface in centimetres. The horizontal axes are (a) temperature in ${ }^{\circ} \mathrm{C}$, (b) salinity in ppt and (c) brine volume fraction as a percentage. 
(a) Horizontal resistivities

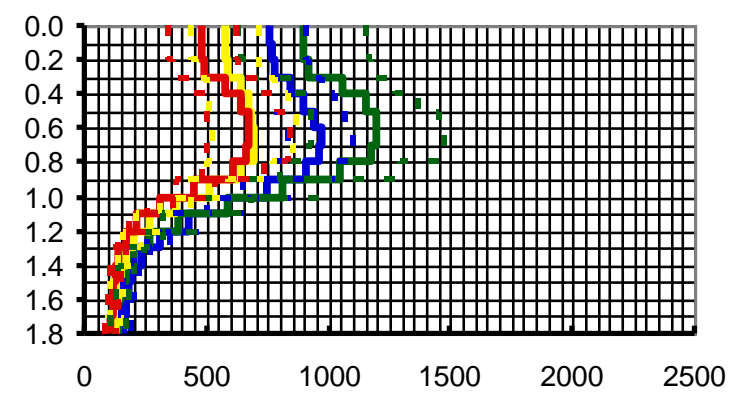

(c) Geometric mean resistivities

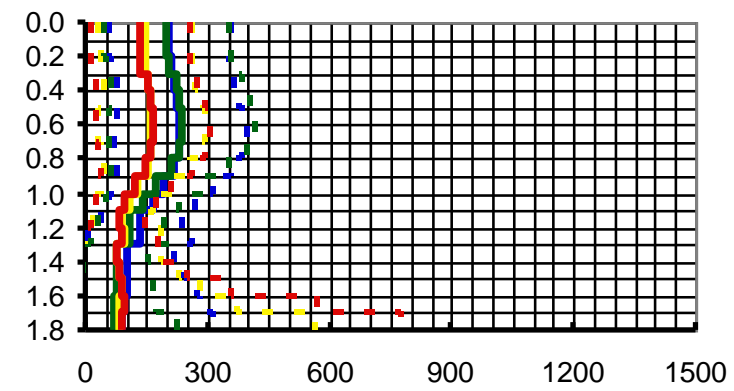

(b) Vertical resistivities

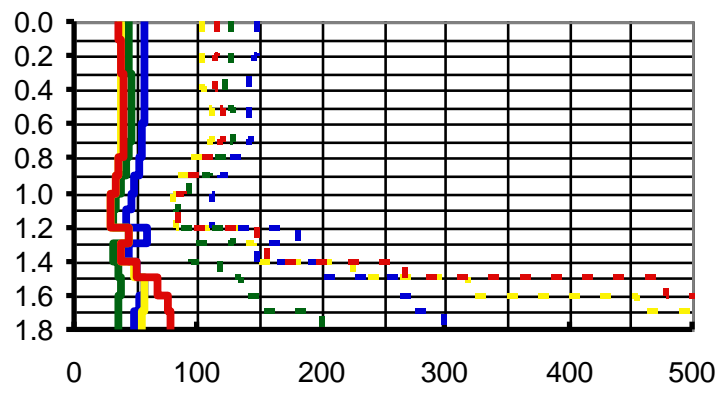

(d) Coefficient of anisotropy

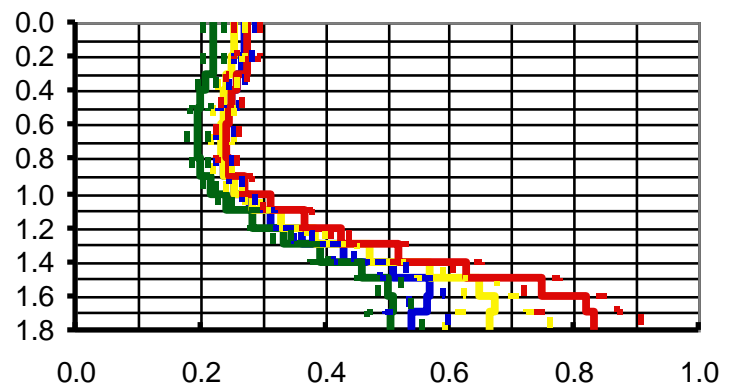

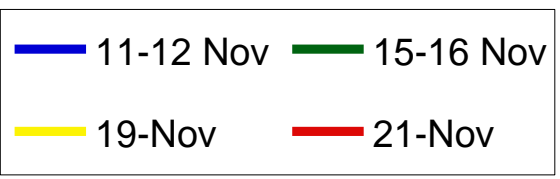

Figure 5.12: Line plots of average 2009 data values with depth, for (a) the horizontal resistivity, (b) the geometric mean resistivity, (c) the vertical resistivity and (d) the coefficient of anisotropy of all four data sets. The vertical axes are depth from surface in meters and the horizontal axes are resistivity in ohm meters except (d) where the horizontal axis is the coefficient of anisotropy. Dashed lines indicate estimates of the uncertainties in the values.

The vertical sections of the different components of the bulk resistivity, as shown in Figure 5.9b $\left(\rho_{m}\right)$, Figure 5.10a $\left(\rho_{H}\right)$ and Figure 5.10b $\left(\rho_{v}\right)$, show little variation over the short two week measurement period, where temperatures did not change significantly. The relatively constant values for each component of the bulk resistivity are also clear in the line plots of Figure 5.12. The ice thickness at the time of all the measurements $(\sim 2.41 \mathrm{~m})$ was greater than the length of the electrode strings $(1.8 \mathrm{~m})$, thus the ice/seawater interface is not observed.

Figure 5.10a and Figure 5.12a show that the horizontal component of the bulk resistivity displays values of between 100 and $1200 \mathrm{ohm}$ meters. The horizontal resistivity profiles increase slightly with depth, flatten off then below about $0.8 \mathrm{~m}$ there is a significant decrease in resistivity. The slightly lower surface resistivities may indicate a region of the initial granular or frazil growth of the ice cover. The resistivities in the middle 
region of the profiles vary from $650 \Omega \mathrm{m}$ to $1200 \Omega \mathrm{m}$, similar to values observed in the early part of the sequence of the 2008 Arctic measurements (Figure 5.4a). However, there are no significant changes in resistivity observed in the Arctic models, until the ice/seawater interface. There is no significant variation in either temperature or brine volume fraction to account for large changes in resistivity. For example, between $0.8 \mathrm{~m}$ and $1.5 \mathrm{~m}$ depth the calculated brine volume fraction is relatively constant with values of between $2.5 \%$ and $3.5 \%$, and while there is a slight rise in temperature from about $-10^{\circ} \mathrm{C} /-8^{\circ} \mathrm{C}$ at $0.8 \mathrm{~m}$ depth to $-7^{\circ} \mathrm{C} /-6^{\circ} \mathrm{C}$ at $1.5 \mathrm{~m}$ depth, this is not regarded as large enough to result in significant temperature controlled changes in microstructure. Such changes are predicted (e.g. Golden et al., 1998, 2007 and Golden, 2003) to occur when the brine volume fraction reaches $5 \%$ and the temperature $-5{ }^{\circ} \mathrm{C}$.

In the Antarctic platelet ice can form beneath the ice cover in supercooled water, creating a tumbled, entwined platelet matrix. This matrix can be incorporated into the ice cover as it grows downwards (Leonard et al., 2006; McGuiness et al., 2009; Dempsey et al., 2010). Such a region of included platelet ice could be expected to have a greater degree of horizontal connectivity between brine inclusions, than that seen in vertically aligned columnar ice. The greater horizontal connectivity would be observed as a decrease in the horizontal component of resistivity. Thus, rather than representing a change in ice microstructure with temperature, we suggest that the observed resistivity decrease indicates a change in actual ice type from columnar ice to incorporated platelet ice. At depths above $0.8 \mathrm{~m}$ there is a predominance of columnar ice, with little horizontal connectivity between brine inclusions. Beneath this depth the ice type changes reaching platelet ice, with greater horizontal connectivity of brine, near the base of the resistivity profiles.

Compared to columnar ice, a region which includes platelet ice could also be expected to have less vertical connectivity between brine inclusions. This decrease in vertical connectivity would be observed as an increase in the vertical resistivity of the ice cover. There is a change in the vertical resistivity profiles around a depth of $1.2 \mathrm{~m}$, with a slight increase in resistivities below this depth. However, it would seem that despite the difference in the structure of the ice crystals the vertical connectivity of the individual brine inclusions is similar throughout. 
The suggested change in ice type can also be observed in plots of the coefficient of anisotropy (Figure 5.10c and Figure 5.12d), with a significant increase in $\lambda$ below $0.8 \mathrm{~m}$ as the value of the horizontal and vertical resistivities become more similar in value. This indicates a change from an anisotropic brine structure in a region of columnar ice to a more isotropic structure in a region which includes platelet ice.

Studies of platelet ice in McMurdo Sound have found; platelet ice to be incorporated into the ice cover below depths of $0.68 \mathrm{~m}$ (Leonard et al., 2006) to 0.8m (Smith et al., 1998), and to predominate below depths of $1.1 \mathrm{~m}$ (Leonard et al., 2006). Over the course of the 2009 Antarctic winter, participants of Antarctica New Zealand science event K131 made repeat CTD casts through the ice. These identified significant periods when platelet ice was present in the water column beneath the growing ice cover. Furthermore, analysis of ice cores, taken as part of Antarctica New Zealand science event K131, indicates the following sea ice structure; surface frazil ice (above $0.1 \mathrm{~m}$ ) followed by aligned columnar ice to a depth of $1 \mathrm{~m}$, below this is disrupted columnar ice (with smaller vertical extent) containing the occasional platelet at $1.5 \mathrm{~m}$, and finally platelet ice is observed below $1.75 \mathrm{~m}$ (Gough, A. pers. comm.). These depths are similar to those obtained from the resistivity structure. Thus the core data support the interpretation of the resistivity data and the suggestion that these data differentiates between columnar and incorporated platelet ice. 



\section{Chapter 6 \\ Investigating brine structures}

The bulk resistivity of the sea ice depends upon brine resistivity, brine volume fraction $\left(V_{b} / V\right)$ and the structure of the brine inclusions. Defining formation factor (FF) as bulk resistivity over brine resistivity $\left(\rho / \rho_{b}\right)$ combines these variables, so that a plot of formation factor versus brine volume fraction should give some initial indication of any changes that occur in the ice microstructure. The concept of formation factor derives from Archie's Law (Archie, 1942) as shown in section 6.1 below.

Figure 6.1 displays four plots of FF $\left(\rho / \rho_{b}\right)$ versus brine volume fraction $\left(V_{b} / V\right)$, showing the horizontal and vertical components of the bulk resistivity for the six measurement sets in the Arctic and the four measurement sets in the Antarctic. The resistivities are obtained from inversion of the data, while the brine resistivities are found using the measured temperature data and the expression given by Stogryn (1971) (see section 3.9). The brine volume fractions can be found from measured temperature and salinity data using the expressions of Cox and Weeks (1983) (see section 3.7).

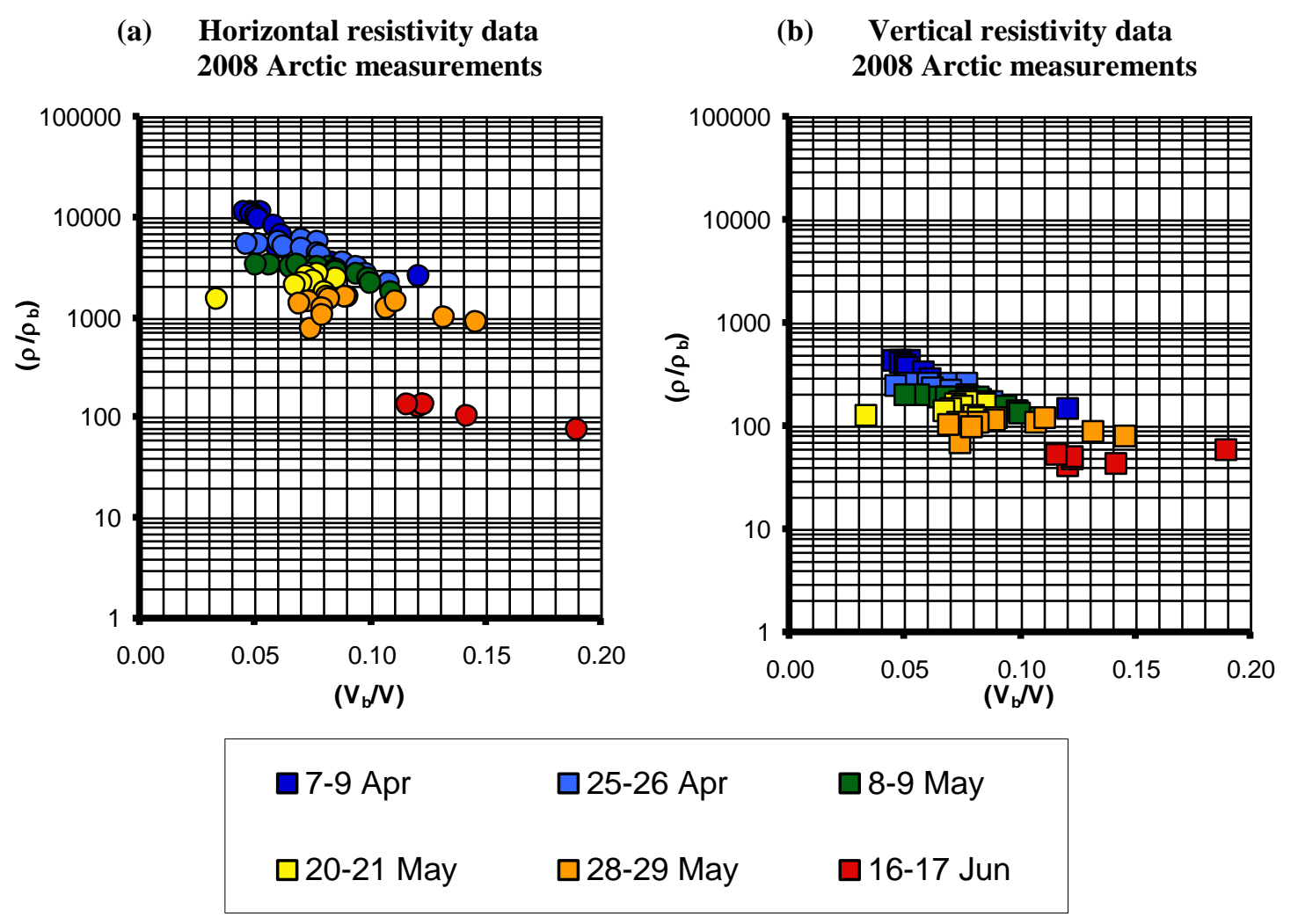


(c) Horizontal resistivity data 2009 Antarctic measurements

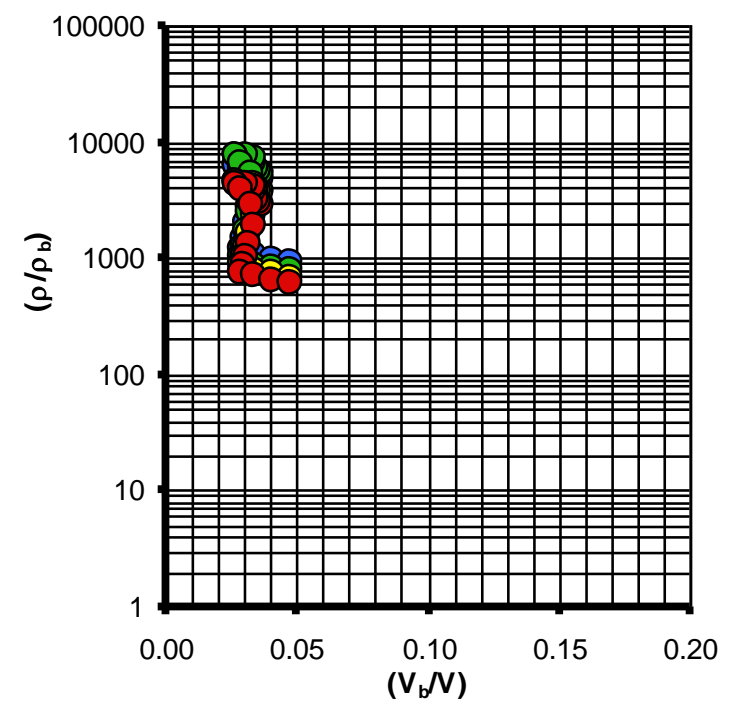

(d) Vertical resistivity data 2009 Antarctic measurements

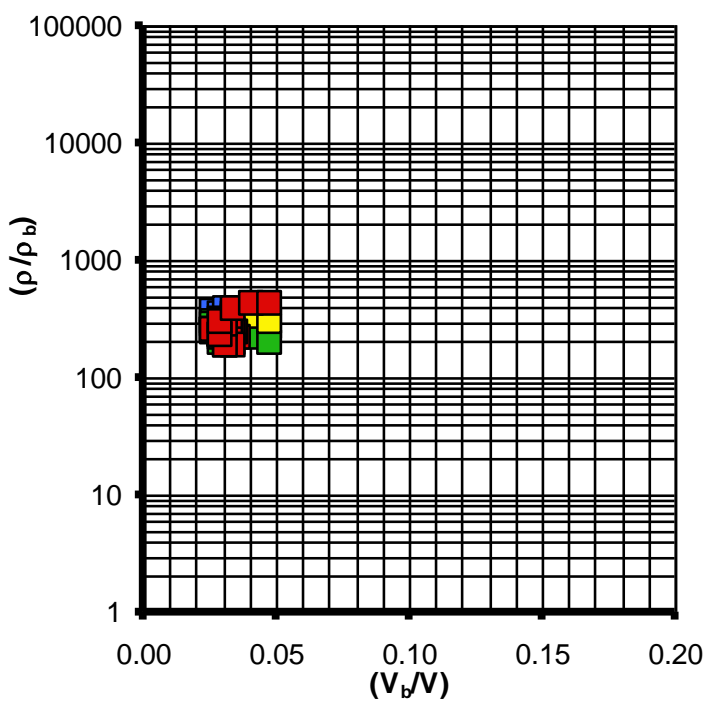

\section{1-12 Nov $\square 15-16$ Nov $\square 19-N o v \quad \square 21-N o v$}

Figure 6.1: Plots of formation factor $\left(\rho / \rho_{b}\right)$ vs. brine volume fraction $\left(V_{b} / V\right)$ for $(a)$ the horizontal resistivity data from the 2008 Arctic measurements (b) the vertical resistivity data from the 2008 Arctic measurements, (c) the horizontal resistivity data from the 2009 Antarctic measurements and (d) the vertical resistivity data from the 2009 Antarctic measurements.

In the two plots for the Arctic datasets (Figure 6.1a for the horizontal resistivity and Figure $6.1 \mathrm{~b}$ for the vertical resistivity) it can be seen that there is a decrease in $\mathrm{FF}\left(\rho / \rho_{b}\right)$ and an increase in brine volume fraction $\left(V_{b} / V\right)$ as the temperatures increased over the measurement period. In the two plots for the Antarctic datasets (Figure 6.1c for the horizontal resistivity and Figure 6.1d for the vertical resistivity) it can be seen that there is much less variation in the FFs and brine volume fractions than that seen in the Arctic data. This is largely due to the smaller number of Antarctic datasets, measured over a shorter time period, during which the temperature changed only slightly.

Within both the Arctic and Antarctic data sets there is an obvious decrease in the horizontal FF. In the Arctic data this change is observed over time, and a sharp drop in the horizontal component of resistivity between late May and mid June appears as a sharp discontinuity in the FF plot (Figure 6.1a). This is possibly indicative of a fundamental change in the microstructure of the ice and suggests that a percolation threshold (see section 6.5) may have been crossed. On the other hand the decrease in horizontal FF in the Antarctic data (Figure 6.1c) occurs in all data sets and is most likely 
caused by the suggested change with depth from columnar to platelet or frazil ice mentioned in Chapter 5.

The vertical FF plots (Figure 6.1b for the Arctic data and Figure 6.1d for the Antarctic data) show less of a change than observed in the horizontal FF plots (Figure 6.1a for the Arctic data and Figure 6.1c for the Antarctic data). However, the Arctic data still shows a decrease in vertical FF or resistivity between the late May and mid June measurements. On the other hand, the vertical FF plot of the Antarctic data shows no clear trend. As was also indicated in Chapter 3 (section 3.7) these FF vs. brine volume plots show that the brine volume fractions of the Antarctic ice are lower and have a smaller range of values than that observed in the Arctic ice during our measurement periods.

Many authors (eg Fricke, 1924; Hashin and Shtrikman, 1962; Tinga et al., 1973; Vant et al. 1978; Timco, 1979; Sihvola and Kong, 1988; Chelidze and Gueguen, 1999 and Grimm et al., 2008) have studied the electrical conductivity of multiphase materials (sea ice included) and created models based on mixture theory. Some of these are discussed below with consideration to how well they fit the measured resistivity structure of the first year Arctic and Antarctic sea ice. 


\subsection{Archie's Law}

The variation of bulk resistivity in a two component system, such as sea ice, where the resistivity of one component is much higher than the other, has traditionally been expressed in terms of Archie's Law (Archie, 1942).

$$
\rho=a \phi^{-m} S^{-n} \rho_{b} \text { (from Telford et al., 1977.) }
$$

where $\rho$ is the bulk resistivity of the material, $S$ is the fraction of the pore filled with the more conductive phase, $\phi$ is the porosity, $\rho_{b}$ is the resistivity of the more conductive phase, $n \approx 2$ and $a$ and $m$ are empirically determined constants. For a situation in which all pore spaces are saturated $(S=1)$, this may be expressed as

$$
\rho=a \phi^{-m} \rho_{b}
$$

By rearranging and taking the log of both sides equation (6.1) can then be expressed as

$$
\log \left(\rho / \rho_{b}\right)=\log (a)-m \log (\phi)
$$

As long as the geometry of the microstructure of the medium remains unchanged a $\log -\log$ plot of formation factor against brine volume fraction yields a straight line with intercept $\log (a)$ and slope $-m$. Thus equation (6.2) allows values for the constants $a$ and $m$ to be determined. In the case of sea ice; the more conductive phase is the brine and the porosity $(\phi)$ can be taken as the brine volume fraction $\left(V_{b} / V\right)$, when all pore spaces are saturated. If the brine volume fraction is known then equation (6.2) can be used to calculate the bulk resistivity of the sea ice from the brine resistivity and appropriate values of $a$ and $m$. Conversely if the bulk resistivity is measured this equation could provide the brine volume fraction of the ice.

Originally (6.1) was derived for permeable sandstone displaying no preferred shape or orientation of pores. Morey et al. (1984) note that it had been found that the exponent $m$ was dependent on the shape of the host particles, with $m$ equal to 1.5 for spherical particles, greater than 1.5 for plate like grains or cylinders whose major axis is perpendicular to an applied electric field, and less than 1.5 when the electric field is parallel to the major axis of plate like grains.

Studies of laboratory grown ice-hydrate systems by Grimm et al. (2008) give exponents of $m=1.7$ and 2.1 for $100 \mathrm{mM}$ and $10 \mathrm{mM}$ of $\mathrm{NaCl}$ respectively, $m=0.8$ for $10 \mathrm{mM} \mathrm{HCl}$ and $m=1$ and 2.5 for $100 \mathrm{mM}$ and $10 \mathrm{mM}$ of $\mathrm{CaCl}_{2}$ respectively. For sea ice the derived 
values of $m$ include; 1.55 for the top of the ice and 1.75 for the bottom found by Morey et al. (1984), a value of 2.2 given by Thyssen et al. (1974) and $m=2.88$ calculated by Ingham et al. (2008).

In theory the intercept of the plot of expression (6.2) occurs when $\log (\phi)=\log \left(V_{b} / V\right)=0$, which means the brine volume fraction $\left(V_{b} / V\right)$ is $100 \%$. When the brine volume fraction is $100 \%$ the bulk resistivity $(\rho)$ would equal the brine resistivity $\left(\rho_{b}\right)$ and the formation factor $\left(\rho / \rho_{b}\right)$ would be 1 . If this holds then the intercept of the log-log plot should in fact be equal to zero. However, Archie's Law was determined on laboratory samples in which the porosity (in the case of sea ice this can be taken as the brine volume fraction) ranged from $10 \%$ to $40 \%$ (Archie, 1942). Thus the behaviour of a medium with porosity greater than $40 \%$ can not be assumed to conform to Archie's Law and the statement above, regarding porosity of $100 \%$, may not hold

In order to see how our data are fit by Archie's law plots of $\log \left(\rho / \rho_{b}\right)$ versus $\log \left(V_{b} / V\right)$, showing the horizontal and vertical components of the bulk resistivity for the six measurement sets in the Arctic (Figure 6.2) and the four measurement sets in the Antarctic (Figure 6.3) are shown below.

For the Arctic data, with no constraint on the intercept, the gradient $(m)$ is 1.93 and 1.32 for horizontal (Figure 6.2a) and vertical (Figure 6.2b) resistivity plots respectively. The value for the horizontal resistivity data is above $m=1.5$ which suggests plate like grains whose major axis is perpendicular to an applied electric field. This is consistent with the expected structure of preferentially vertically elongated brine inclusions with the electric field applied in the horizontal direction. The vertical resistivity $m$ value is also consistent with the expectation of vertically elongated brine inclusions. However, this time $m$ is less than 1.5 indicating that the applied electric field is parallel (rather than perpendicular) to the major axes of the plate like grains or cylinders.

Note that only the data up to 28-29 May have been fit by a straight line. Due to a large jump in resistivity after this date the data deviates from a straight line fit. Similarly in their study of the horizontal resistivity of sea ice, Ingham et al. (2008) observed that their data was only consistent with Archie's Law until the brine volume fraction exceeded $8 \%-10 \%$. 
(a)

Horizontal resistivity data 2008 Arctic measurements

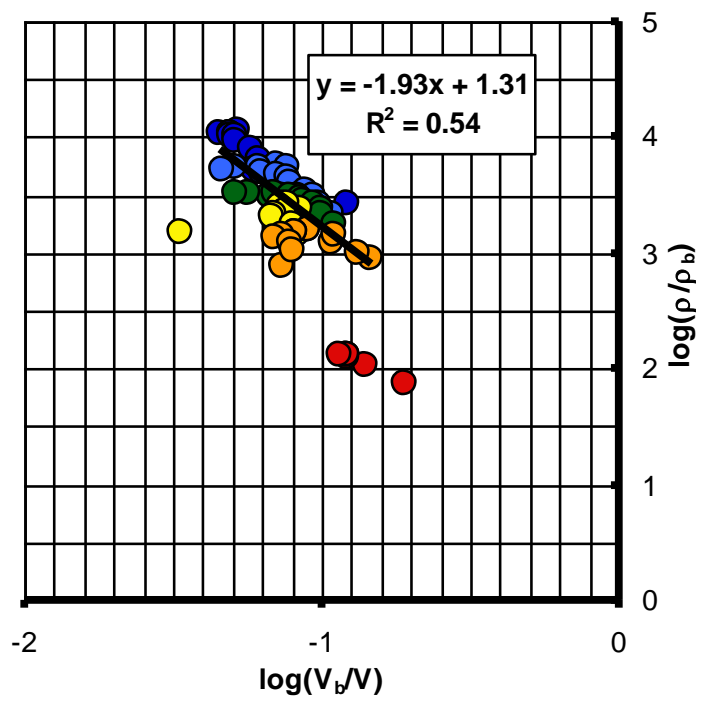

(b)

Vertical resistivity data 2008 Arctic measurements

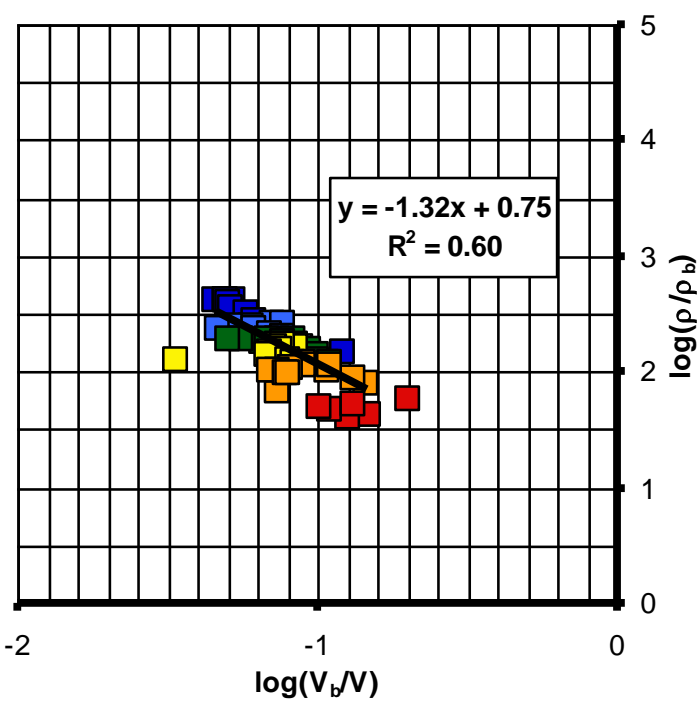

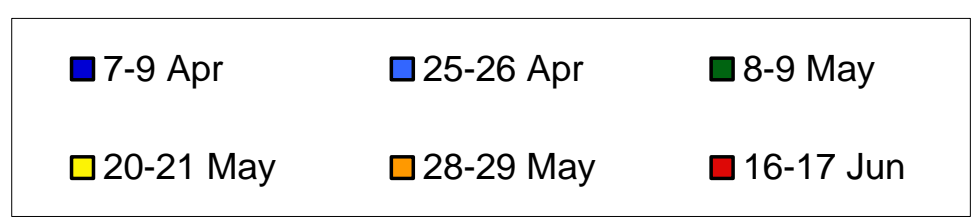

Figure 6.2: $\mathrm{Log}-\log$ plots of formation factor $\left(\rho / \rho_{\mathrm{b}}\right)$ vs. brine volume fraction $\left(\mathrm{V}_{\mathrm{b}} / \mathrm{V}\right)$, showing Archie's Law fit to the 2008 Arctic data. (a) the horizontal resistivity data (b) the vertical resistivity data.

Recall that for the Antarctic data a significant decrease in resistivity below $\sim 0.8 \mathrm{~m}$ is associated with a change in ice type from mainly columnar to included platelet ice. This change in the geometry of the structure means that the Archie's law straight line cannot be fit to all the data together. Instead each of these ice types is considered separately, fitting a straight line to the data above $0.8 \mathrm{~m}$ and similarly to the data below $1.5 \mathrm{~m}$ (as shown in Figure 6.3 below). From ice cores (Gough, A., pers. comm., 2011) the region between $0.8 \mathrm{~m}$ and $1.5 \mathrm{~m}$ is most likely composed of columnar ice whose growth is disrupted by the introduction of the occasional platelet. With no constraint on the intercept, the data above a depth of $0.8 \mathrm{~m}$ (Figure 6.3a) gives $m=0.84$ and $m=0.11$ for the horizontal and vertical resistivity respectively. Below $1.5 \mathrm{~m}$ (Figure 6.3b) $m$ values for the horizontal and vertical resistivities are 0.42 and 0.27 respectively.

However, the data within these two regions of the ice are not fit well by straight lines. This is not surprising, as (i) the exact extent of the different ice types are unknown and there are most likely regions of mixed structure at the boundaries; and (ii) the limited number of data confined within a small range of brine volume fractions means that the gradients are poorly defined. Hence, little can be inferred from $m$ values obtained from 
the FF vs $V_{b} / V$ plots of the Antarctic data. Despite this, attempting to fit Archie's law to the data clearly shows that there is change in the microstructure of the Antarctic sea ice with depth.

Horizontal resistivity data 2009 Antarctic measurements

(a) structure above $0.8 \mathrm{~m}$

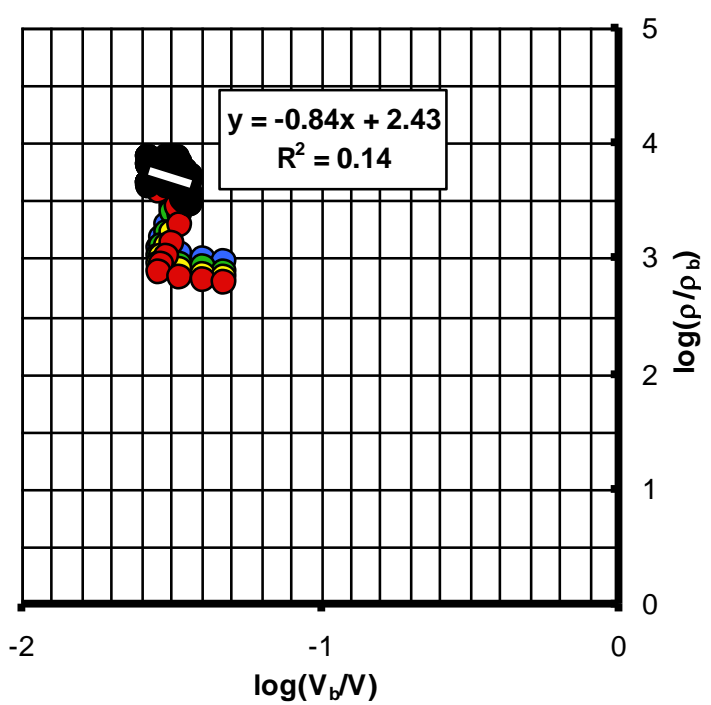

(b) structure below $1.5 \mathrm{~m}$

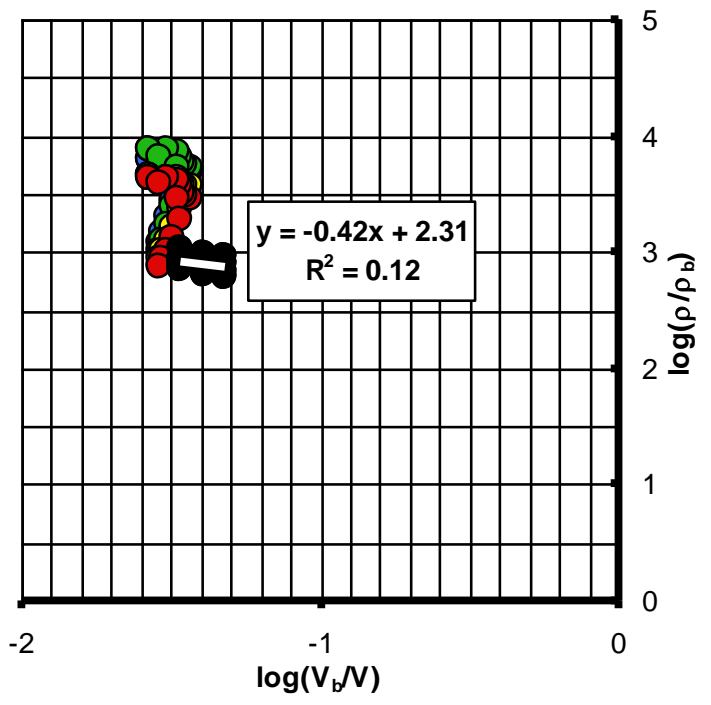

Vertical resistivity data 2009 Antarctic measurements
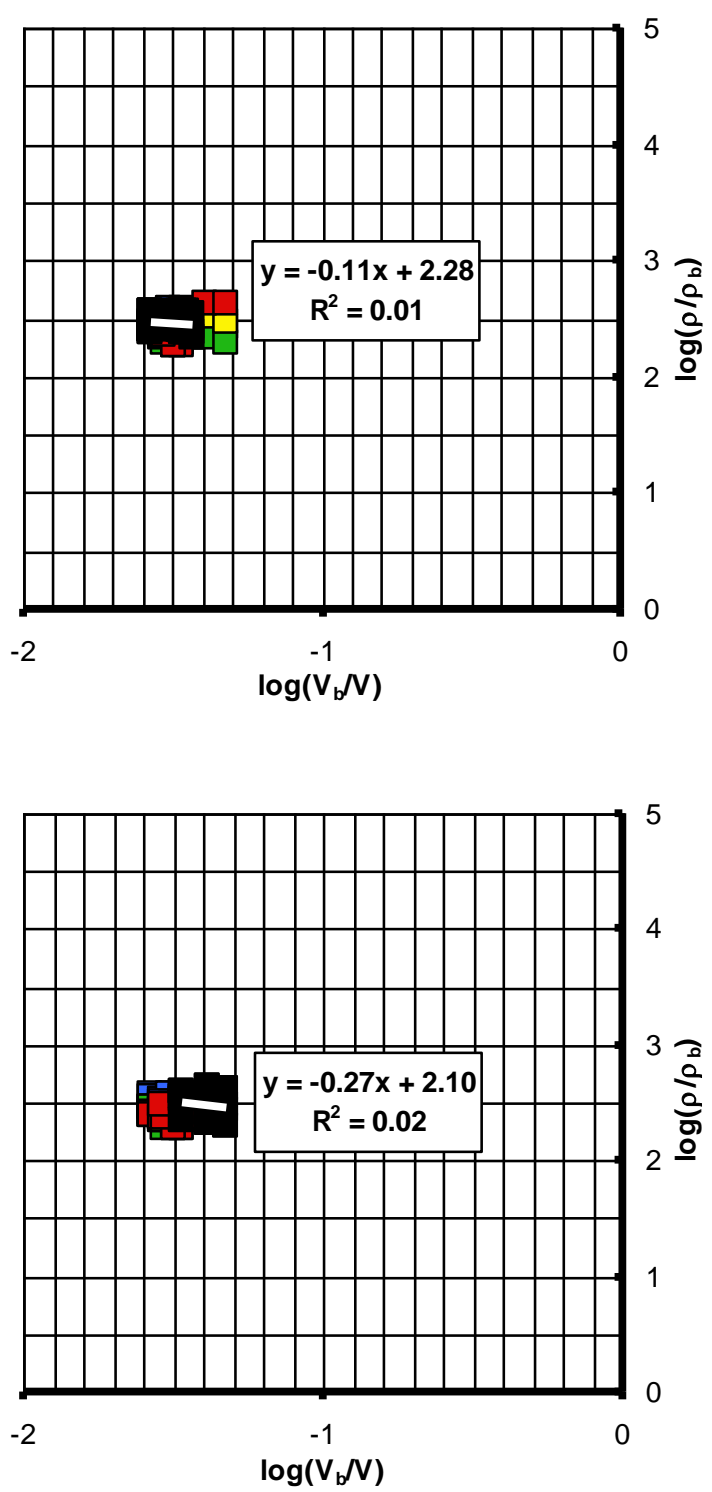

\section{1-12 Nov $\square 15-16$ Nov $\square 19-N o v \quad \square 21-N o v$}

Figure 6.3: Log-log plots of formation factor $\left(\rho / \rho_{\mathrm{b}}\right)$ vs. brine volume fraction $\left(\mathrm{V}_{\mathrm{b}} / \mathrm{V}\right)$, showing Archie's Law fit to the 2009 Antarctic data. (a) resistivity data from above $0.8 \mathrm{~m}$ marked in black, left hand plot horizontal resistivity, right hand plot vertical resistivity. (b) resistivity data from below $1.5 \mathrm{~m}$ marked in black, left hand plot horizontal resistivity, right hand plot vertical resistivity. Archie's Law is fitted to the black data points. 


\subsection{Power law mixing models}

Power law mixing models assume each phase is interconnected with efficiency depending on an exponent $m$. Grimm et al. (2008) compare measurements of electrical properties of ice-hydrate binary systems with mixing models, and provide the following expression for the general power law for two phases

$$
\sigma^{1 / m}=\Phi \sigma_{1}^{1 / m}+(1-\Phi) \sigma_{2}^{1 / m}
$$

where $\Phi$ is the volume fraction of the medium with conductivity $\sigma_{l}$, and the exponent $m$ is commonly between 1 and 3 .

When $m=1$ in expression (6.3) the Maxwell Garnett expression, which considers isolated inclusions in a connected host medium, is approximated. The formulation of the Maxwell Garnett expression involves an exact calculation of the field induced in the uniform host by a single spherical or ellipsoidal inclusion and an approximate treatment of its distortion by the electrostatic interaction between the different inclusions.

The refractive index model (Birchak et al., 1974) is equivalent to (6.3) if $m=2$. Birchak et al. (1974) were investigating probes for measuring soil moisture and produced an expression equivalent to a volumetric average of the complex index of refraction, hence the index of refraction mixing model. This model arises from considering an inhomogeneous mixture consisting of a homogeneous matrix of one material in which particles of a second material are embedded (the particle size being small compared to the wavelength of the electromagnetic wave), the effective propagation constant may be obtained by partitioning into respective contributions from each of the two materials in question.

The Looyenga model (Looyenga, 1965) is recovered from (6.3) if $m=3$. This model was created from reconsideration of previous equations for the dielectric constant of a heterogeneous system. The model considered by Looyenga (1965), consists of spheres of differing radii, in a homogeneous electric field.

Expression (6.3) gives Archie's law with the empirical constant $a$ equal to one if $\sigma_{2}=0$. In this case the intercept of expression (6.2) would be zero and $\sigma_{1}$ would be the conductivity of the more conductive phase. 
Formation factor $\left(\rho / \rho_{b}\right)$ vs. brine volume fraction $\left(V_{b} / V\right)$ plots showing fit of power laws to the Arctic data

(a) Horizontal resistivity data

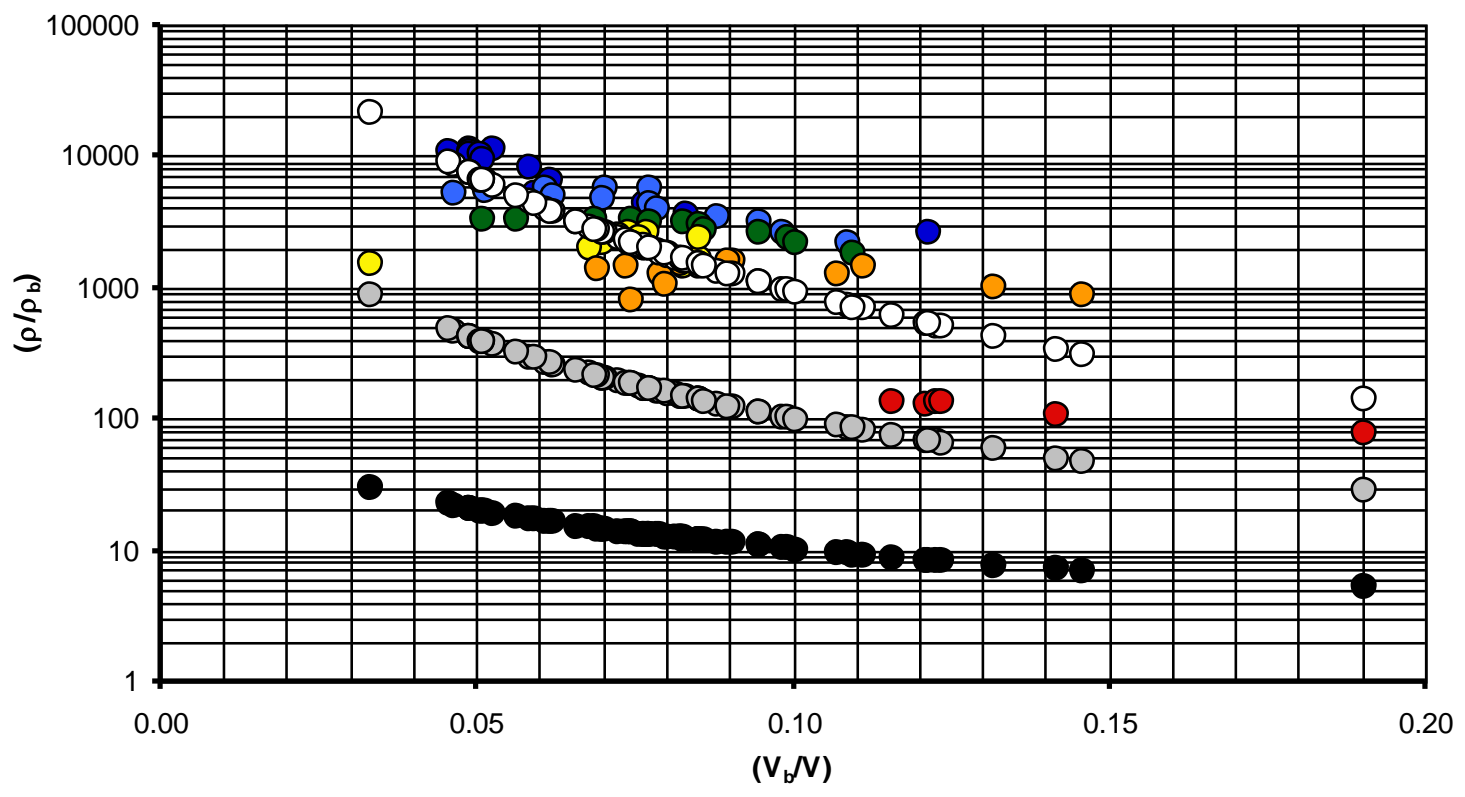

(b) Vertical resistivity data

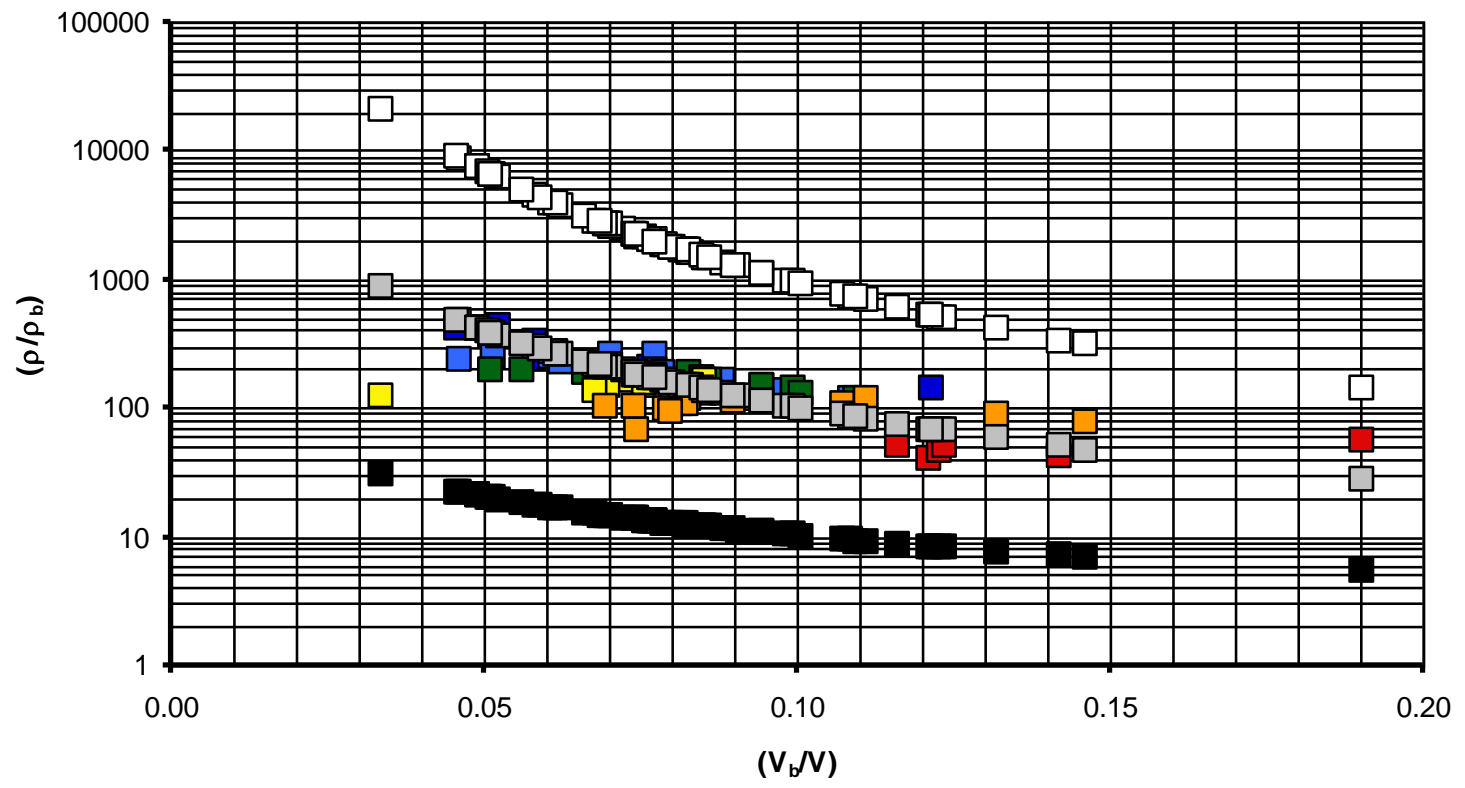

\begin{tabular}{|c|c|c|c|c|}
\hline 7-9 Apr & 口25-26 Apr & ๑ 8-9 May & $\square$ 20-21 May & $\square$ 28-29 May \\
\hline$\square$ 16-17 Jun & D $m=1$ & $\square m=2$ & $\square m=3$ & \\
\hline
\end{tabular}

Figure 6.4: Plots of formation factor $\left(\rho / \rho_{b}\right)$ vs. brine volume fraction $\left(V_{b} / V\right)$ for Arctic data showing power law trends for different $m$ values. (a) Horizontal resistivity data. (b) Vertical resistivity data. 
Formation factor $\left(\rho / \rho_{b}\right)$ vs. brine volume fraction $\left(V_{b} / V\right)$ plots showing fit of power laws to the Antarctic data

(a) Horizontal resistivity data

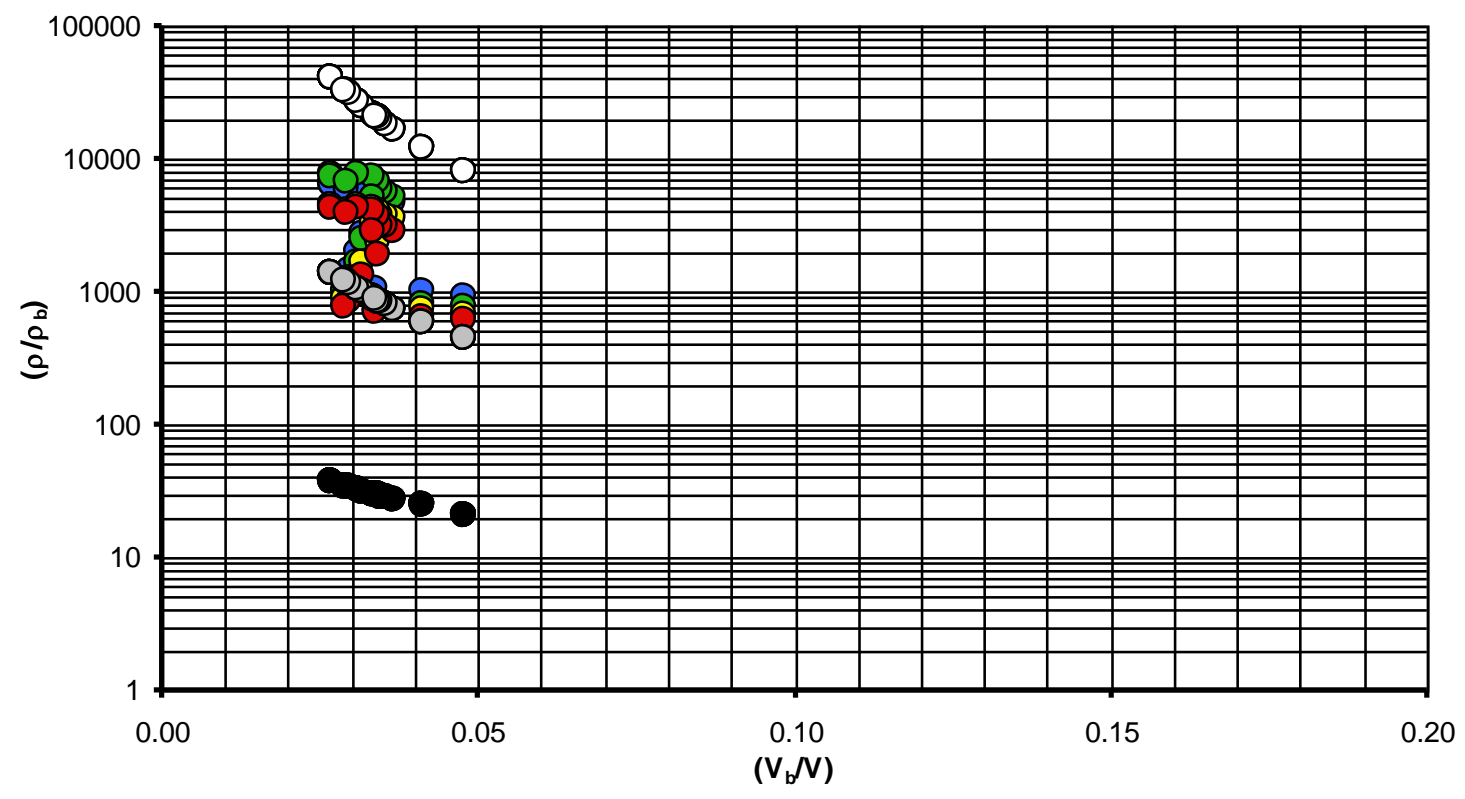

(b) Vertical resistivity data

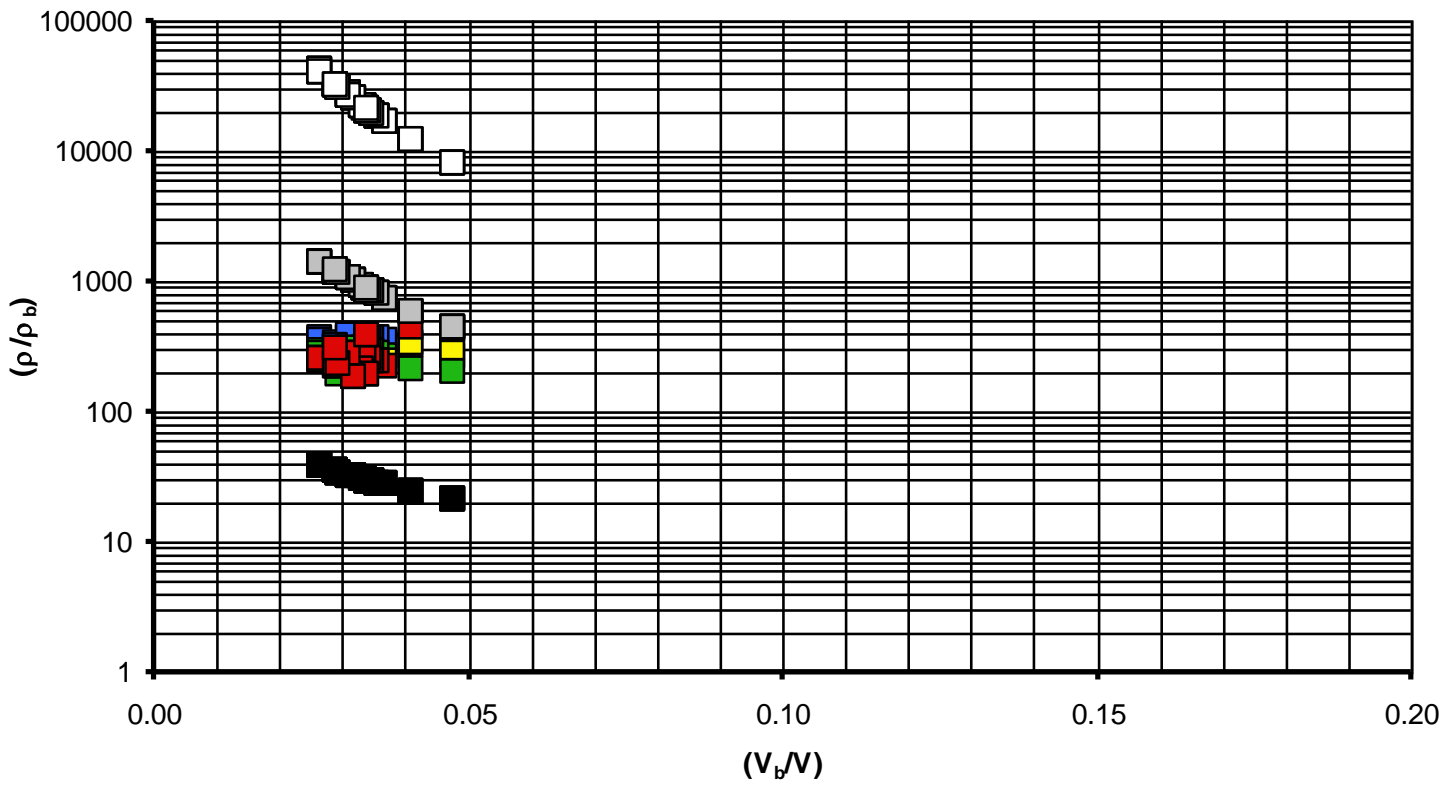

\begin{tabular}{|c|c|c|}
\hline$\square 11-12$ Nov & $\square 15-16$ Nov & $\square 19-N o v$ \\
\hline $\mathrm{m}=1$ & $\square m=2$ & $\square m=3$ \\
\hline
\end{tabular}

Figure 6.5: Plots of formation factor $\left(\rho / \rho_{b}\right)$ vs. brine volume fraction $\left(V_{b} / V\right)$ for Antarctic data, showing power law trends for different $m$ values. (a) Horizontal resistivity data. (b) Vertical resistivity data. 
Using a high value for the resistivity of the ice $(10,000,000 \Omega \mathrm{m}$ - the DC conductivity of pure ice noted by Mathews and Clark (1963)) and setting the brine resistivities and brine volume fractions to those calculated from the measured data, expression (6.3) returns conductivities that can be compared to the measured data. Figure 6.4 and Figure 6.5 above show the fit of the power law mixing models to the FF vs. $V_{b} / V$ data for the vertical and horizontal resistivity measurements in both the Arctic and Antarctic.

The best fit to the horizontal Arctic data (Figure 6.4a) occurs when $m=3$ i.e. the Looyenga model. On the other hand, the best fit to the vertical Arctic data (Figure 6.4b) is achieved when $m=2$ or the refractive index model is applied. However both these fits are rough and, importantly, no single model explains both the horizontal and vertical component of the bulk resistivity.

Fitting the power law mixing models to the Antarctic data is complicated by the fact that the brine structure changes with depth. Figure 6.5 shows that for the Antarctic data both the horizontal and vertical data are most closely matched when $m=2$ or the refractive index model is used. However, this model only fits the lower resistivity region of the horizontal data, i.e. the region suggested to include platelet ice. The resistivities measured in the upper regions of the ice, where the suggested structure is mostly columnar, are closer in value to those obtained when $m=3$. However, the Looyenga model does not provide a good fit to these data either, and gives higher resistivities than those measured. The match to the vertical Antarctic data is even worse with all measurements below the FF (or resistivity) suggested by the refractive index model.

The fit of the power law mixing models can of course be affected by the conductivity and volume fraction values used in the calculations. The brine volume fractions and brine resistivities are obtained from calculations involving the measured data so should be of an acceptable value. However, the conductivity of the solid ice matrix is not well constrained with various values suggested by the literature. Increasing the conductivity of the ice will decrease the resistivity of the medium. This decrease is more pronounced in the Looyenga model $(m=3)$ but all models show little change until the resistivity of the ice is lowered to $1000 \Omega \mathrm{m}$. This resistivity seems unrealistically low, considering most of the salts are rejected from the ice lattice. Thus the plots obtained above are applicable for a range of realistic ice resistivities. 


\subsection{Suspensions of spheroids}

Fricke (1924) considers the electrical conductivity of a suspension of homogeneous non-polarisable spheroids, deriving an expression for the bulk conductivity of the medium. Comparisons are made with experimental data for the conductivity of the blood of a dog and suspensions of sand in salted gelatine. The expression given by Fricke (1924), describing the conductivity of this kind of system is

$$
\frac{\beta V_{2}}{\left(1-V_{2}\right)}=\left(\frac{\sigma-\sigma_{1}}{\sigma_{2}-\sigma}\right)\left(\frac{\sigma_{2}}{\sigma_{1}}-1\right)
$$

where $\sigma$ is the conductivity of the suspension, $\sigma_{l}$ is the conductivity of the suspending medium, $\sigma_{2}$ is the conductivity of the suspended medium, $V_{2}$ is the volume concentration of the suspended medium (i.e. in the case of sea ice a brine volume fraction), and $\beta$ is given by

$$
\beta=\frac{1}{3}\left\{\frac{2}{1+\left(\frac{\sigma_{2}}{\sigma_{1}}-1\right) \frac{M}{2}}+\frac{1}{1+\left(\frac{\sigma_{2}}{\sigma_{1}}-1\right)(1-M)}\right\}\left\{\frac{\sigma_{2}}{\sigma_{1}}-1\right\}
$$

where $M$ is given, for oblate spheroids $(a<b)$, by

$$
M=\frac{\phi-\frac{1}{2} \sin 2 \phi}{\sin ^{3} \phi} \cos \phi \quad \text { where } \cos \phi=a / b
$$

and for prolate spheroids $(a>b)$ by

$$
M=\frac{1}{\sin ^{2} \phi}-\frac{\cos ^{2} \phi}{2 \sin ^{3} \phi} \log \left(\frac{1+\sin \phi}{1-\sin \phi}\right) \quad \text { where } \cos \phi=b / a
$$

In both cases $a$ is half the length of the axis considered parallel to the direction of the electric force and $b$ half the length of each of the remaining two axes.

Rearranging (6.4) above gives the following expression for the conductivity of the suspension

$$
\sigma=\sigma_{1}\left(\frac{\left(1-V_{2}\right)\left(\sigma_{2}-\sigma_{1}\right)+\beta V_{2} \sigma_{2}}{\left(1-V_{2}\right)\left(\sigma_{2}-\sigma_{1}\right)+\beta V_{2} \sigma_{1}}\right)
$$

Timco (1979) presents the results and analysis of 66 Schlumberger geoelectric soundings on first year sea ice. This includes consideration of a conductivity model for 
the sea ice. The chosen model consists of conducting spheroids in a poorly-conducting matrix. Timco (1979) notes that "Clearly such a model is very highly idealized and should not be interpreted as a rigorous representation of sea-ice structure. Nevertheless, it is felt that such an approach does provide useful information with regard to the typical characteristics of brine cells in sea ice". Following Fricke (1924) the author gives the conductivity of a system of prolate spheroids (with the electric field parallel to the long axis of the spheroid) as

$$
\sigma=\frac{\sigma_{1}\left[\sigma_{1}+\left\{A\left(1-V_{2}\right)+V_{2}\right\}\left(\sigma_{2}-\sigma_{1}\right)\right]}{\sigma_{1}+A\left(1-V_{2}\right)\left(\sigma_{2}-\sigma_{1}\right)}
$$

where $\sigma_{1}$ is the conductivity of the ice matrix, $\sigma_{2}$ is the conductivity of the brine inclusions, $V_{2}$ is the brine volume in parts per thousand (i.e. a brine volume fraction), and $A$ is given by

$$
A=\frac{-1}{(l / 2 b)^{2}-1}+\frac{(l / 2 b)}{\left\{(l / 2 b)^{2}-1\right\}^{3 / 2}} \ln \left[\frac{l}{2 b}+\left\{(l / 2 b)^{2}-1\right\}^{1 / 2}\right]
$$

where $l$ is the length of the brine cell and $b$ the average radius of the brine cells.

From the field measurements Timco (1979) obtains values of vertical resistivity equal to $76 \Omega \mathrm{m}, V_{2}=63 \%, \sigma_{l}=3 \times 10^{-5} \mathrm{~S} / \mathrm{m}, \sigma_{2}=5 \mathrm{~S} / \mathrm{m}$ and $b=0.046 \mathrm{~mm}$, these then provide an average value for $l$ of $1.7 \mathrm{~cm}$.

Tinga et al. (1973) provide a brief overview of previous studies of dielectric mixture theory and look at finding the complex dielectric constant of a multiphase mixture with confocal ellipsoidal shell inclusions. They simplify their results to consider two phase structures with ellipsoidal inclusions, giving the following expression

$$
\frac{\varepsilon-\varepsilon_{1}}{\varepsilon_{1}}=\frac{V_{2}}{V_{1}} \frac{\varepsilon_{2}-\varepsilon_{1}}{\varepsilon_{1}+n_{2}\left(\varepsilon_{2}-\varepsilon_{1}\right)-\left(\frac{n_{1} V_{2}}{V_{1}}\right)\left(\varepsilon_{2}-\varepsilon_{1}\right)}
$$

where $\varepsilon_{i}$ is the dielectric constant of medium $i, V_{2} / V_{1}$ is the volume fraction of medium 2 , the $n_{i}$ are depolarisation coefficients.

This expression can be rearranged to give an expression for the electrical permittivity of the medium. 


$$
\varepsilon=\varepsilon_{1}\left\{1+\frac{V_{2}}{V_{1}} \frac{\varepsilon_{2}-\varepsilon_{1}}{\varepsilon_{1}+n_{2}\left(\varepsilon_{2}-\varepsilon_{1}\right)-\left(\frac{n_{1} V_{2}}{V_{1}}\right)\left(\varepsilon_{2}-\varepsilon_{1}\right)}\right\}
$$

Using Debye theory the complex permittivity is given by

$$
\varepsilon^{*}=\varepsilon_{\infty}+\frac{\varepsilon_{l}-\varepsilon_{\infty}}{1+i \omega \tau}-\frac{i \sigma}{\omega \varepsilon_{o}}
$$

where $\varepsilon_{l}$ and $\varepsilon_{\infty}$ are the low and high frequency limits of $\varepsilon^{*}, \omega$ is the frequency, $\tau$ the relaxation time, $\sigma$ the conductivity and $\varepsilon_{o}$ the permittivity of free space.

Additionally the complex conductivity is given by

$$
\sigma^{*}=i \omega \varepsilon_{o} \varepsilon^{*}=i \omega \varepsilon_{o} \varepsilon_{\infty}+i \omega \varepsilon_{o} \frac{\varepsilon_{l}-\varepsilon_{\infty}}{1+i \omega \tau}+\sigma
$$

Hence

$$
\frac{\sigma_{a} *}{\sigma_{b} *}=\frac{i \omega \varepsilon_{o} \varepsilon_{a} *}{i \omega \varepsilon_{o} \varepsilon_{b} *}=\frac{\varepsilon_{a}^{*}}{\varepsilon_{b} *}
$$

and

$$
\frac{\sigma_{a} *}{\sigma_{b} *}=\frac{i \omega \varepsilon_{o} \varepsilon_{a \infty}+i \omega \varepsilon_{o} \frac{\varepsilon_{a l}-\varepsilon_{a \infty}}{1+i \omega \tau_{a}}+\sigma_{a}}{i \omega \varepsilon_{o} \varepsilon_{b \infty}+i \omega \varepsilon_{o} \frac{\varepsilon_{b l}-\varepsilon_{b \infty}}{1+i \omega \tau_{b}}+\sigma_{b}}
$$

For DC where $\omega=0$ these give

$$
\frac{\varepsilon_{a}^{*}}{\varepsilon_{b} *}=\frac{\sigma_{a}}{\sigma_{b}} \Rightarrow \varepsilon_{a}^{*}=\frac{\sigma_{a}}{\sigma_{b}} \varepsilon_{b} *
$$

Using this, the expression for the dielectric constant (or permittivity) above can be rewritten to give the conductivity as

$$
\sigma=\sigma_{1}\left\{1+\frac{V_{2}}{V_{1}} \frac{\sigma_{2}-\sigma_{1}}{\sigma_{1}+n_{2}\left(\sigma_{2}-\sigma_{1}\right)-\left(\frac{n_{1} V_{2}}{V_{1}}\right)\left(\sigma_{2}-\sigma_{1}\right)}\right\}
$$

Vant et al. (1978) carried out measurements of the dielectric constant of sea ice samples and compared these with previously described dielectric models. They suggest that the expression put forward by Tinga et al. (1973), for a two phase structure with ellipsoidal 
inclusions, may have particular promise. The authors state the in the case of ellipsoidal inclusion $n_{1}$ and $n_{2}$ are equal and given by

$$
n=\left(\frac{b}{a}\right)^{2}\left(\frac{1}{4 e^{3}}\right)\left(\frac{2 e}{(b / a)^{2}}+\ln \frac{1-e}{1+e}\right)
$$

when the electric field is applied along the short axes $(2 b)$ of the ellipsoid and by

$$
n=\left(\frac{b}{a}\right)^{2}\left(\frac{1}{2 e^{3}}\right)\left(-2 e+\ln \frac{1+e}{1-e}\right)
$$

when the electric field is applied along the long axis $(2 a)$ of the ellipsoid $e$ is the ellipsoid eccentricity defined by

$$
e=\left[1-(b / a)^{2}\right]^{1 / 2}
$$

Thus the conductivity of the medium is given by the following expression, obtained from (6.7) with $n_{1}=n_{2}=n$

$$
\sigma=\sigma_{1}\left\{1+\frac{V_{2}}{V_{1}} \frac{\sigma_{2}-\sigma_{1}}{\sigma_{1}+n\left(\sigma_{2}-\sigma_{1}\right)\left(1-\left(\frac{V_{2}}{V_{1}}\right)\right)}\right\}
$$

The expressions suggested by Fricke (1924) consider both prolate and oblate spheroids with the electric field applied parallel to the long axis of the prolate spheroids and the short axis of the oblate spheroids. Timco (1979), however, only considers the case of the electric field being parallel to the long axis of prolate spheroids. Tinga et al. (1973) and Vant et al. (1978) allow for consideration of electric fields applied parallel and perpendicular to the long axis of prolate spheroids.

Considering first year sea ice, where the brine inclusions are preferentially elongated in the vertical direction and there is little or no horizontal anisotropy, the prolate spheroid models seem to be a better approximation to the structure. The horizontal resistivity is thus obtained when the electric field is along the short axis of the spheroid inclusions and, the vertical resistivity when the electric field is parallel to the long axis. The case of the oblate spheroid (Fricke (1924)) does not represent the suggested brine structure of first year sea ice and, therefore, is not considered further here. It should be noted that it is not necessary to set values for the length of the axes as, given the conductivities of 
the spheroid and background media, it is the axes ratio that determines the resistivity structure.

The expressions obtained from Tinga et al. (1973) and Vant et al. (1978) are shown on FF vs. $V_{b} / V$ plots in Figure 6.6 (Arctic data) and Figure 6.7 (Antarctic data) below, along with the measured horizontal and vertical resistivity data. Conductivities are obtain from expression (6.10) using (6.9) to give the vertical conductivity and (6.8) to give the horizontal conductivity. Values for the conductivity of the spheroid inclusions, which represent the brine component, are obtained from calculations involving the temperature of the ice during measurements. The conductivity of the background medium, which represents the ice matrix, is set to $0.0000001 \mathrm{Sm}^{-1}(10,000,000 \Omega \mathrm{m})$, the DC conductivity of pure ice as noted by Mathews and Clark (1963). However, the conductivity of the solid ice matrix is not well constrained with a range of values found within the literature. Thus the plots display results for differing axes ratios and the effect of changing the conductivity of the background medium or ice matrix.

The values obtained from Tinga et al. (1973) and Vant et al. (1978) for the vertical resistivity show essentially the same results as would be obtained from Timco's expression, derived from Fricke (1924). Timco (1979) used a conductivity of $3 \times 10^{-5}$ $\mathrm{S} / \mathrm{m}$ for the ice and derived lengths of $b=0.046 \mathrm{~mm}$ and $l=2 a=1.7 \mathrm{~cm}$. The results of using these values, which give a ratio of $b / a=0.0054$, in the expressions of Tinga et al. (1973) and Vant et al. (1978) have also been displayed on the vertical resistivity plots below. 
Formation factor $\left(\rho / \rho_{b}\right)$ vs. brine volume fraction $\left(V_{b} / V\right)$ plots showing Tinga et al. (1973) and Vant et al. (1978) expressions for the conductivity of a suspension of prolate spheroids

(a) Horizontal resistivity data (Arctic data)

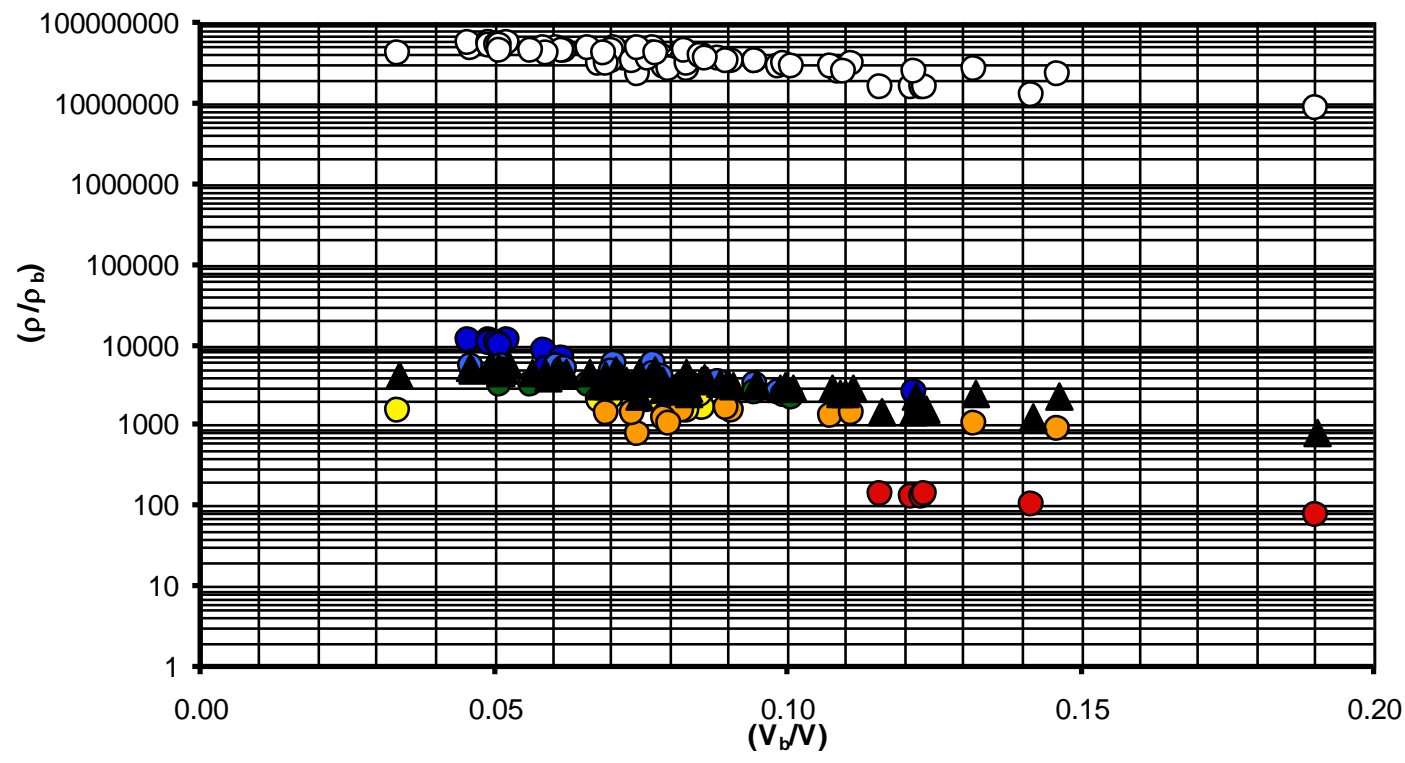

- 7-9 Apr

O 20-21 May

O conductivity of ice $=0.0000001 \mathrm{~S} / \mathrm{m}$
0 25-26 Apr
O 28-29 May
0 8-9 May
0 16-17 Jun

(b) Vertical resistivity data (Arctic data)

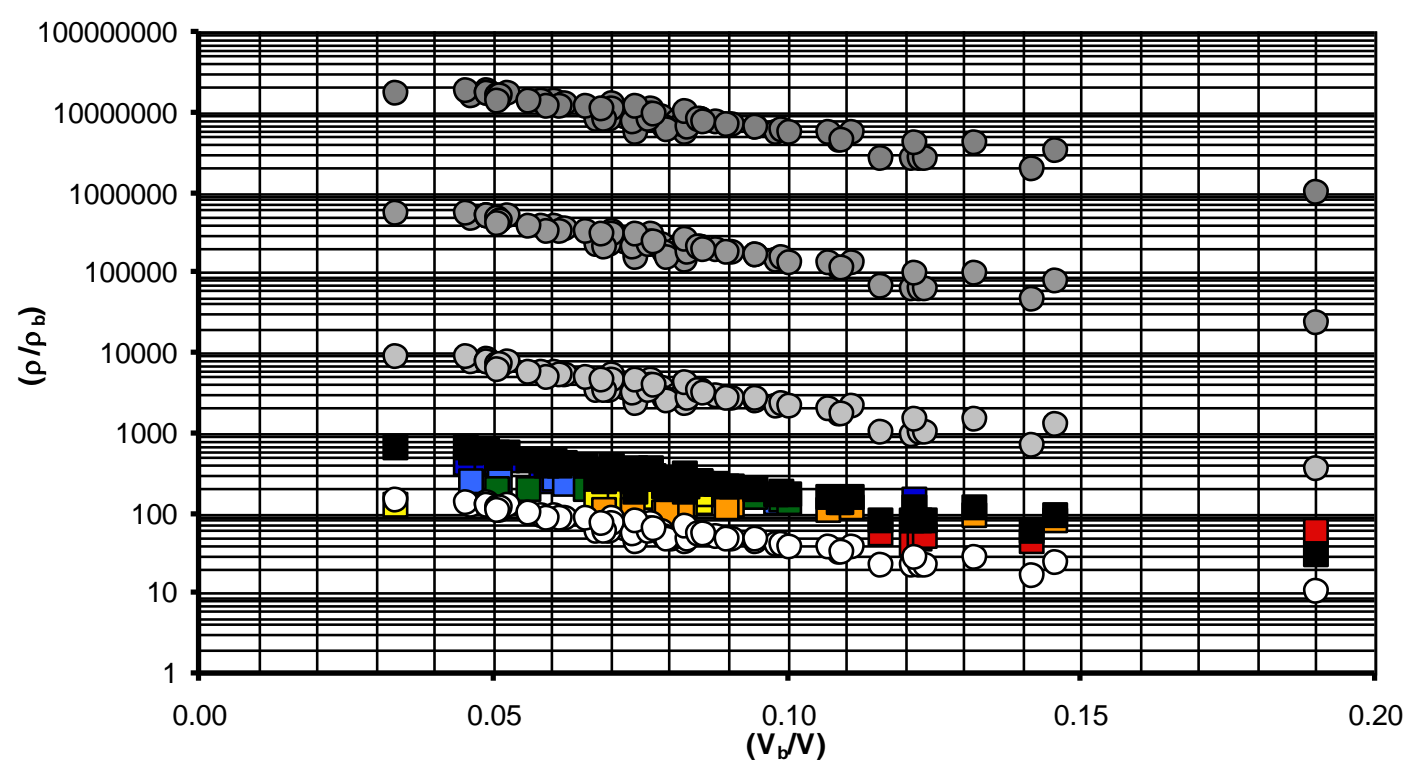

$\begin{array}{llllll}\square 7-9 \text { Apr } & \square 25-26 \text { Apr } & \square 8-9 \text { May } & \square 20-21 \text { May } & \square 28-29 \text { May } & \square 16-17 \text { Jun } \\ \text { O b/a=0.1 } & \text { ob/a=0.01 } & \text { Ob/a=0.001 } & \text { Ob/a=0.0001 } & \text { ロTimco } 1979\end{array}$

Figure 6.6: Plots of formation factor $\left(\rho / \rho_{b}\right)$ vs. brine volume fraction $\left(V_{b} / V\right)$ for Arctic data, showing Tinga et al. (1973) and Vant et al. (1978) expressions for the conductivity of a suspension of prolate spheroids. (a) Horizontal resistivity data. (b) Vertical resistivity data. 
Formation factor $\left(\rho / \rho_{b}\right)$ vs. brine volume fraction $\left(V_{b} / V\right)$ plots

showing Tinga et al. (1973) and Vant et al. (1978) expressions for the conductivity of a suspension of prolate spheroids

(a) Horizontal resistivity data (Antarctic data)

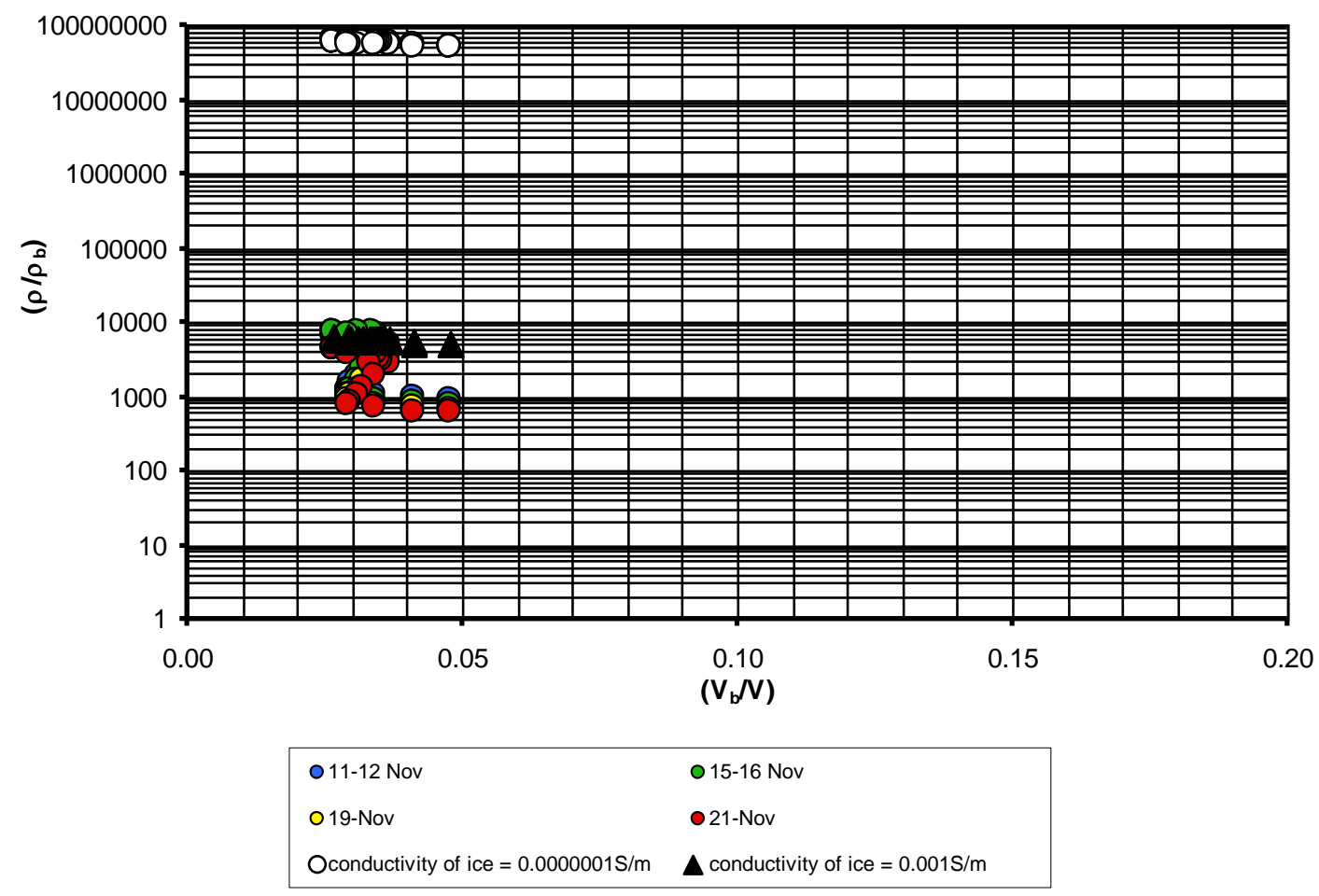

(b) Vertical resistivity data (Antarctic data)

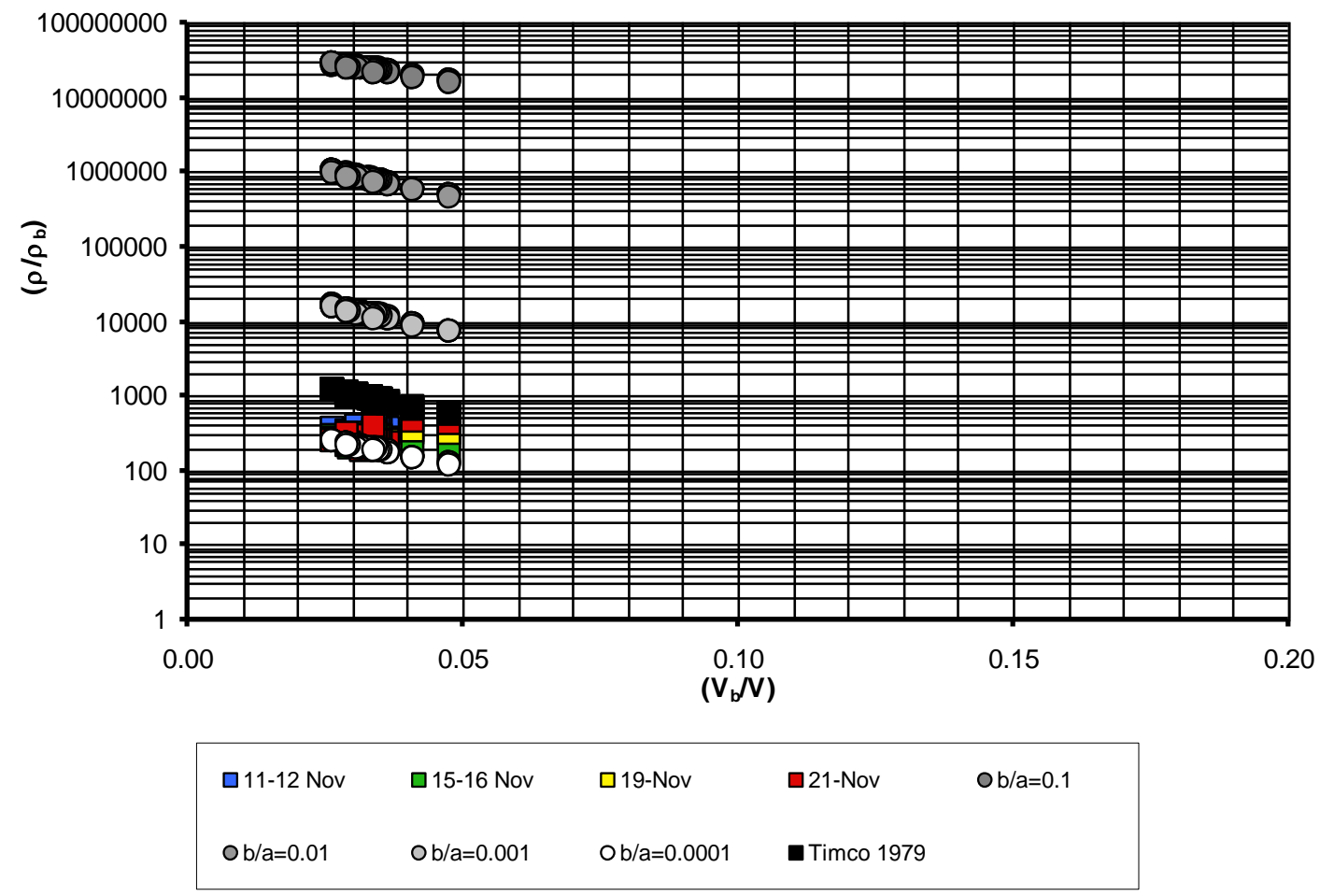

Figure 6.7: Plots of formation factor $\left(\rho / \rho_{\mathrm{b}}\right)$ vs. brine volume fraction $\left(\mathrm{V}_{\mathrm{b}} / \mathrm{V}\right)$ for Antarctic data, showing Tinga et al. (1973) and Vant et al. (1978) expressions for the conductivity of a suspension of prolate spheroids. (a) Horizontal resistivity data. (b) Vertical resistivity data. 
From Figure 6.6a and Figure 6.7a, showing the horizontal resistivity for the Arctic and Antarctic data respectively, it can be seen that by considering brine inclusions in the form of prolate spheroids and a low conductivity for the ice matrix the expressions suggested by Tinga et al. (1973) and Vant et al. (1978) do not match the resistivity data measured in the field. All axes ratios $(b / a)$ return comparable conductivities and thus only one curve is visible on the plots. The only way to obtain similar values to the measurements is to significantly increase the conductivity of the ice $\left(\sim 0.001 \mathrm{Sm}^{-1}\right)$. Such a high conductivity seems unrealistic as it is generally accepted that most of the salts are rejected from the ice lattice as the seawater freezes. Furthermore even if such a value for the ice conductivity were used the shape of the theoretical formation factor curve is significantly flatter than the observed trends, especially the Antarctic data.

Figure $6.6 \mathrm{~b}$ and Figure 6.7b, displaying the vertical Arctic and Antarctic data respectively, shows that the suggested model provides a match to the measured data if the ratio of the axes $(b / a)$ is between 0.001 and 0.0001 , when the ice conductivity is $0.0000001 \mathrm{Sm}^{-1}$. With a higher conductivity value for the ice matrix the resistivity of the medium would of course decrease. In this case the ratio $b / a$ would need to be higher, i.e. the length of the short axis $(b)$ becomes closer to the length of the long axis $(a)$, for the vertical resistivity values to be similar to that obtained by measurement.

Using the values of ice conductivity and the b/a ratio stated by Timco (1979) the expressions of Tinga et al. (1973) and Vant et al. (1978) provide values similar to those obtained from measurements of the vertical resistivity of Arctic sea ice (see Figure 6.6b). For the Antarctic data the values used by Timco give higher FF values than observed in the measured data (see Figure 6.7b).

From these results it is seen that considering a suspension of homogeneous prolate spheroids does not produce a model that closely matches both the vertical and horizontal resistivity structure of first year sea ice. Additionally, as we know nothing of the separation of the spheroids it is difficult to determine much information on the structure, for instance is there any connection between brine inclusions or are they isolated. These types of models only produce an average picture of the brine structure. 


\subsection{Cuboid models}

It is possible to build 3D structures of cuboid inclusions of brine within a matrix of ice, calculate the resistivity as the brine volume fraction changes and compare with the measured data. In this way a simplistic picture of the microstructure of the measured sea ice cover can be developed, giving some insight into changes in the structure with depth and time.

For example consider a structure comprising of isolated cubes of a material with a conductivity of $\sigma_{2}$ within a matrix of a second material with a conductivity of $\sigma_{1}$, as shown in Figure 6.8 for a unit cube of the material.

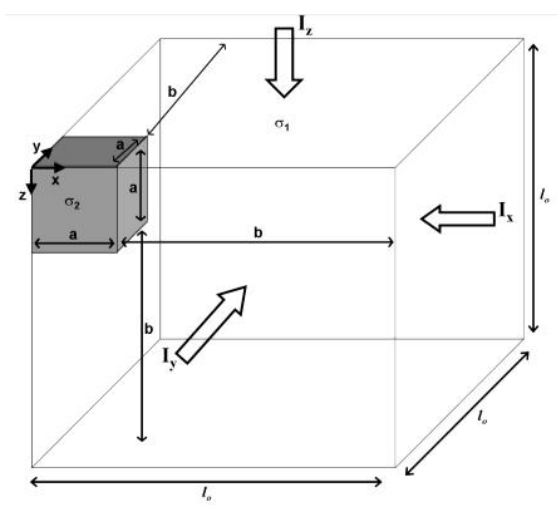

Figure 6.8: Unit cube of a two phase structure, consisting of cubes of a material with conductivity $\sigma_{2}$, within a matrix of material with conductivity $\sigma_{1}$. The distance between the cubes is given by $b$ while the dimensions of the cubes are given by $a$. The direction of the $x, y$ and $z$ axes have been labelled.

For this structure the conductivity measured in the three different directions $(x, y$ and $z)$ will be the same, due to the isotropy of the structure. A bulk conductivity $(\sigma)$ can be found by equating the current expressed in the form of Ohm's Law $(\underline{\mathrm{J}}=\sigma \underline{\mathrm{E}} \Rightarrow I=A \sigma E)$ to the current expressed as the sum of currents through each possible path i.e. current can find a path through just the ice or it can travel through a mixture of brine and ice. In the case of current travelling through both ice and brine the conductivity $\sigma_{e}$ is the effective conductivity of a section which contains both materials. Such a conductivity can be found using the relationship between conductivity and resistance $(\sigma=l /(A R))$, where the resistance $R$ is obtained by adding the resistances of the two separate materials.

From these calculations the bulk conductivity $(\sigma)$ for this cubic structure is given by 


$$
\sigma=\left(1-\frac{a^{2}}{(a+b)^{2}}\right) \sigma_{1}+\frac{a^{2}}{(a+b)} \frac{\sigma_{1} \sigma_{2}}{b \sigma_{2}+a \sigma_{1}}
$$

The volume fraction of the material with a conductivity of $\sigma_{2}$ is given by

$$
\frac{V_{2}}{V}=\frac{a^{3}}{(a+b)^{3}}
$$

Consider the structure shown in Figure 6.8, where the isolated cubes represent brine inclusions and the background matrix solid ice. The side of the unit cube or $a+b$ is set to be constant, this confines the structure so that if the horizontal dimensions of the cubes $(a)$ increase the spacing between the cubes $(b)$ must decrease. Note that the actual values of $a$ and $b$ depend only on the size set for the unit cube. Any multiple of the derived values will give the same formation factor and brine volume so long as the ratio $a / b$ remains the same. The formation factor $\left(\rho / \rho_{b}\right)$ of cubic models with different ice conductivities are calculated over low to $100 \%$ brine volume fraction using equations (6.11) and (6.12) and the brine conductivities calculated from measured temperature data and the expression given by Stogryn (1971). These formation factors (FF) are compared to those calculated from the measured data. Figure 6.9 and Figure 6.10 show plots of FF vs. brine volume fraction for the horizontal and vertical resistivities of cubic models and the Arctic and Antarctic data. 
(a) Horizontal resistivity (Arctic data)

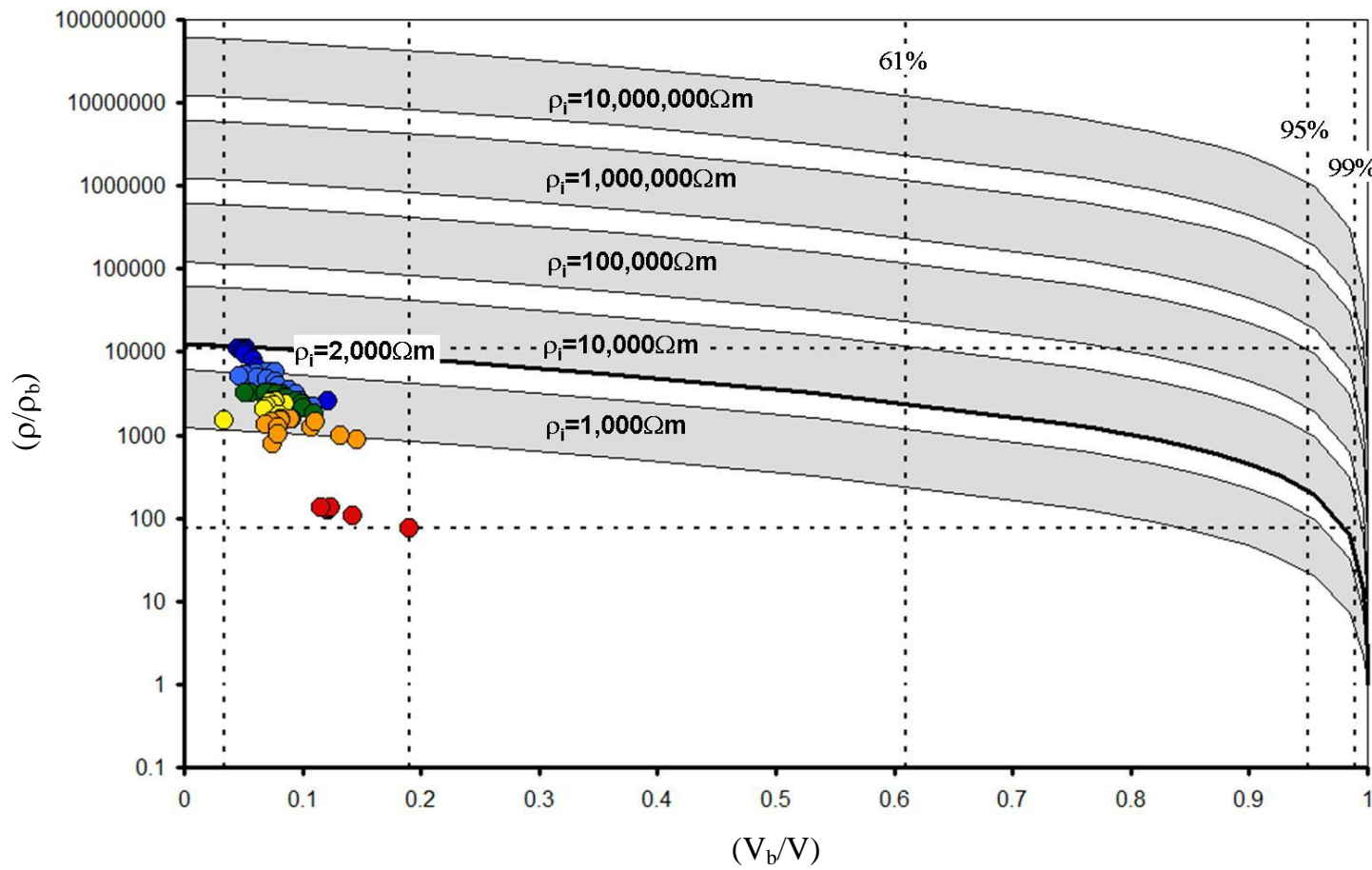

(b) Vertical resistivity (Arctic data)

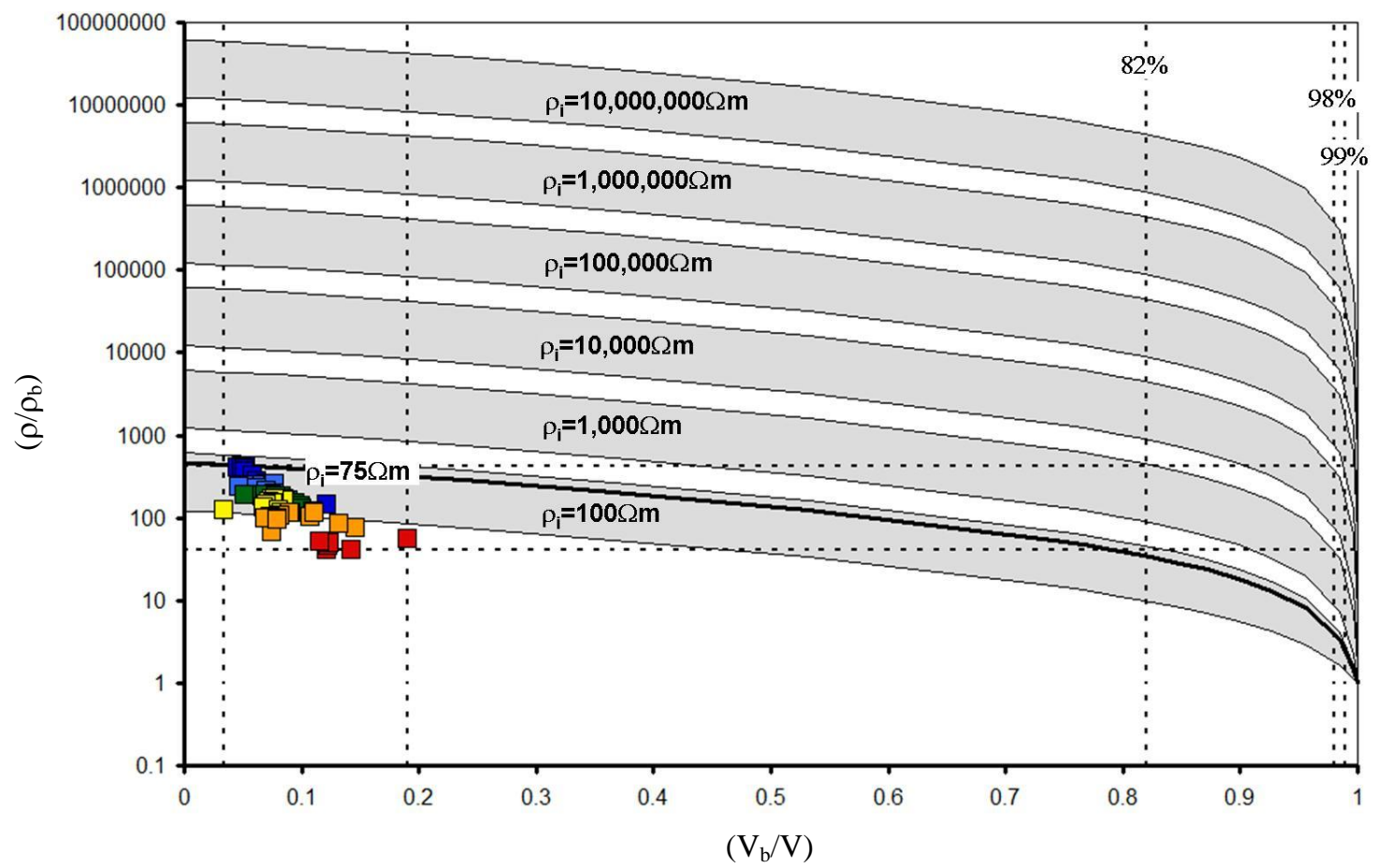

\begin{tabular}{|lll|}
\hline$\square 7-9$ Apr & $\square 25-26$ Apr & $\square 8-9$ May \\
$\square 20-21$ May & $\square 28-29$ May & $\square 16-17$ Jun \\
\hline
\end{tabular}

Figure 6.9: Plots of formation factor $\left(\rho / \rho_{b}\right)$ vs. brine volume fraction $\left(V_{b} / V\right)$ of both the proposed cubic structures (grey shading) and the measured Arctic data (coloured points), for (a) the horizontal resistivity and (b) the vertical resistivity. 
(a) Horizontal resistivity (Antarctic data)

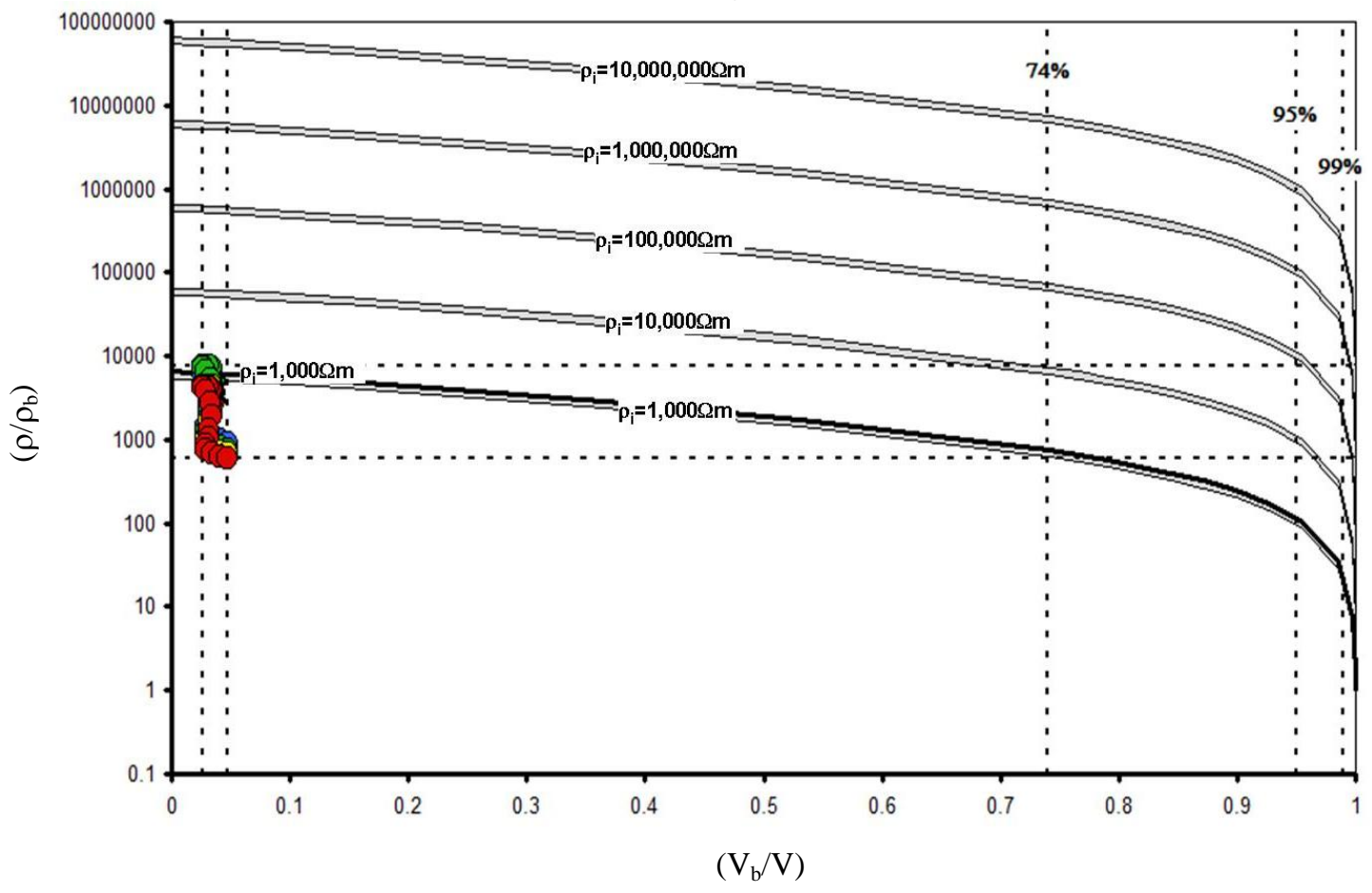

(b) Vertical resistivity (Antarctic data)

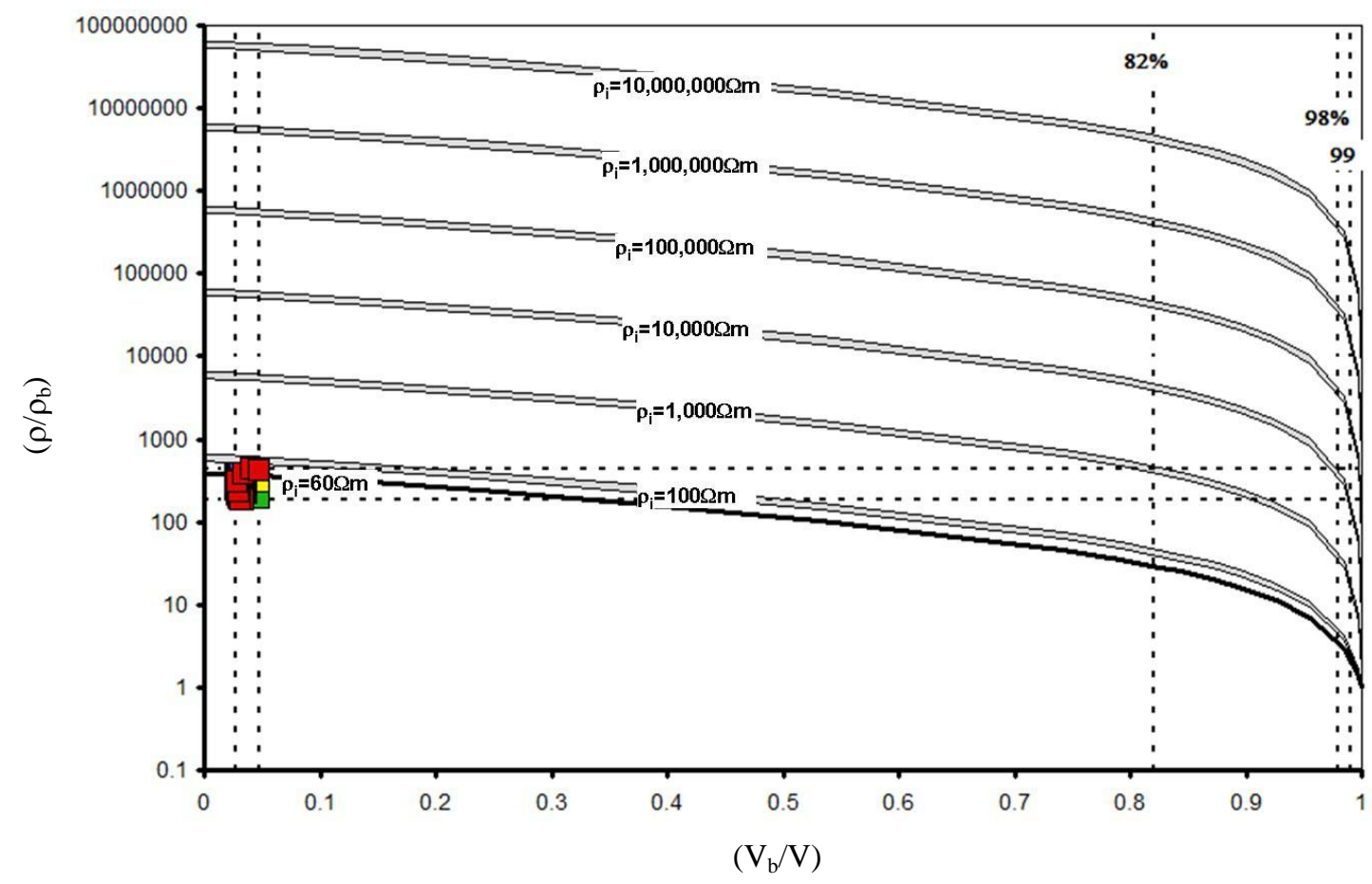

$\square 11-12$ Nov $\square 15-16$ Nov $\square 19-N o v \quad \square 21-N o v$

Figure 6.10: Plots of formation factor $\left(\rho / \rho_{b}\right)$ vs. brine volume fraction $\left(V_{b} / V\right)$ of both the proposed cubic structures (grey shading) and the measured Antarctic data (coloured points), for (a) the horizontal resistivity and (b) the vertical resistivity. 
Initially the conductivity of the ice is set as $\sigma_{l}=0.0000001 \mathrm{Sm}^{-1}(10,000,000 \Omega \mathrm{m})$, the DC conductivity of pure ice as noted by Mathews and Clark (1963). As mentioned above, the conductivity of the solid ice matrix is not well constrained, for example Timco (1979) give $0.00003 \mathrm{Sm}^{-1}(\sim 33,333 \Omega \mathrm{m})$ for the conductivity of a matrix of ice containing trapped impurities. Thus a range of $\sigma_{l}$ values is considered. The shaded areas in Figure 6.9 and Figure 6.10 indicate the range of formation factors obtained for each of the ice conductivities by considering the maximum and minimum calculated brine conductivities.

From the plots of horizontal FF (Figure 6.9a and Figure 6.10a) it can be seen that for ice resistivities of the order of $10,000,000 \Omega \mathrm{m}$ to $1,000,000 \Omega \mathrm{m}$ the FFs do not give values within the range of the required FFs until a brine volume fraction of greater than $99 \%$ is reached. With $\rho_{l}$ of the order of $100,000 \Omega \mathrm{m}$ the brine volume fraction is $95 \%$ before the FFs are with in the calculated range. For the resistivity of ice set at $10,000 \Omega \mathrm{m}$ the FFs are not within the calculated range until the brine volume fraction is greater than $61 \%$ and $74 \%$ for the Arctic and Antarctic data respectively. It is not until the resistivity of the solid ice matrix is of the order of $1,000 \Omega \mathrm{m}$ that a reasonable match to the calculated FFs and brine volume fractions are obtained. In fact the resistivity of the ice must be less than $2000 \Omega \mathrm{m}$ for the Arctic data and less than $1000 \Omega \mathrm{m}$ for the Antarctic data, as shown by the solid black curves in Figure 6.9a and Figure 6.10a. This resistivity seems unrealistically low for the solid ice matrix, especially when one considers that most of the salts are rejected from the ice lattice to brine inclusions as the seawater freezes.

As can be seen in Figure $6.9 \mathrm{~b}$ and Figure $6.10 \mathrm{~b}$ the resistivity of the ice must be even lower, at less than $75 \Omega \mathrm{m}$ for the Arctic data and less than $60 \Omega \mathrm{m}$ for the Antarctic data, for the vertical FF data to be matched. Hence, the plots in Figure 6.9 and Figure 6.10 show that for the cubic structure, shown in Figure 6.8, and what are considered reasonable estimates of the resistivity of the ice, the brine volume fraction must be greater than $95 \%$ in order to achieve the measured vertical and horizontal resistivities. This indicates the need for significant connectivity in both the vertical and horizontal directions in order to obtain the 'low' resistivities measured in the field. 
Hence in order to match the vertical resistivity we consider a structure comprising of vertical cubic columns of a material with a conductivity of $\sigma_{2}$ within a matrix of a second material with a conductivity of $\sigma_{l}$, a unit cube of this material is shown in Figure 6.11 below.

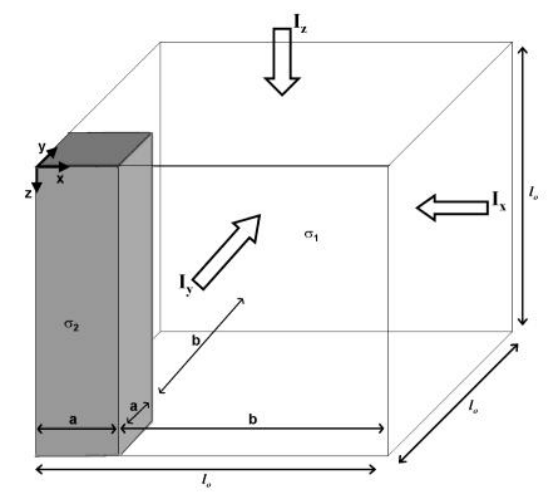

Figure 6.11: Unit cube of a two phase structure, consisting of vertical cubic columns of a material with a conductivity $\sigma_{2}$, within a matrix of material with conductivity of $\sigma_{1}$. The distance between the columns is given by $b$ while the horizontal dimensions of the columns is given by $a$. The direction of the $x, y$ and $z$ axes have been labelled.

For this structure the conductivity measured in the two horizontal directions ( $x$ and $y$ ) will be the same, due to isotropy of the horizontal structure, while that in the vertical direction ( $z$ ) will be different from these. Hence $\sigma_{x}=\sigma_{y}=\sigma_{H}$ and $\sigma_{z}=\sigma_{V}$. Using the same considerations as with the bulk conductivity of the cubic structure previously, the horizontal $\left(\sigma_{H}\right)$ and vertical $\left(\sigma_{V}\right)$ conductivities can be found. Thus it can be seen that the horizontal conductivity $\left(\sigma_{H}\right)$ for this column structure is given by

$$
\sigma_{H}=\frac{b}{(a+b)} \sigma_{1}+\frac{a \sigma_{1} \sigma_{2}}{b \sigma_{2}+a \sigma_{1}}
$$

while the vertical conductivity $\left(\sigma_{V}\right)$ is

$$
\sigma_{V}=\sigma_{1}+\frac{a^{2}}{(a+b)^{2}}\left(\sigma_{2}-\sigma_{1}\right)
$$

The volume fraction of the material with a conductivity of $\sigma_{2}$ is given by the following expression

$$
\frac{V_{2}}{V}=\frac{a^{2}(a+b)}{(a+b)^{3}}=\frac{a^{2}}{(a+b)^{2}}
$$

Take the structure shown in Figure 6.11 as having the vertical cubic columns representing brine ( $\sigma_{2}$ obtained from the brine resistivities calculated from the measured 
data) and the background matrix representing ice $\left(\sigma_{l}=0.0000001 \mathrm{Sm}^{-1}(10,000,000 \Omega \mathrm{m})\right.$, the DC conductivity of pure ice as noted by Mathews and Clark (1963)). If a sensible initial value for $a$ is chosen (suggested dimensions of brine inclusions can be obtained from literature - see section 1.2) and $a+b$ is set to be constant (this confines the structure so that if the horizontal dimensions of the columns $(a)$ increases the spacing between the columns $(b)$ must decrease) a value for $b$ can be deduced so that the vertical formation factors $\left(\rho_{v} / \rho_{b}\right)$, obtained via equation (6.14) and the value of $\sigma_{2}$, match the vertical formation factors (FF) calculated from the measured data. Using the derived values of $a$ and $b$ the brine volume fraction of the proposed ice structure can be found using expression (6.15). It should be noted that the sizes of $a$ and $b$ can be any multiple of the derived values so long as $a / b$ remains the same.

Figure 6.12a and Figure 6.13a show plots of vertical FF vs. brine volume fraction containing the Arctic and Antarctic data respectively. From these graphs it can be seen that the FF (or resistivity) is the same as that obtained from the measured data, however, the brine volume fraction is much lower. This indicates the need for additional brine structure in the model.

For the suggested dimensions ( $a$ and $b$ ) of the proposed column structure the horizontal FF can be obtained using expression (6.13) and the value of $\sigma_{2}$. From the plots of horizontal FF vs. brine volume fraction, as shown in Figure 6.12b (Arctic data) and Figure 6.13b (Antarctic data), it can be seen that not only is the brine volume fraction too low, but also, as expected, the FF is too high as there is no horizontal connectivity in the suggested model. 
(a) Vertical resistivity (Arctic data)

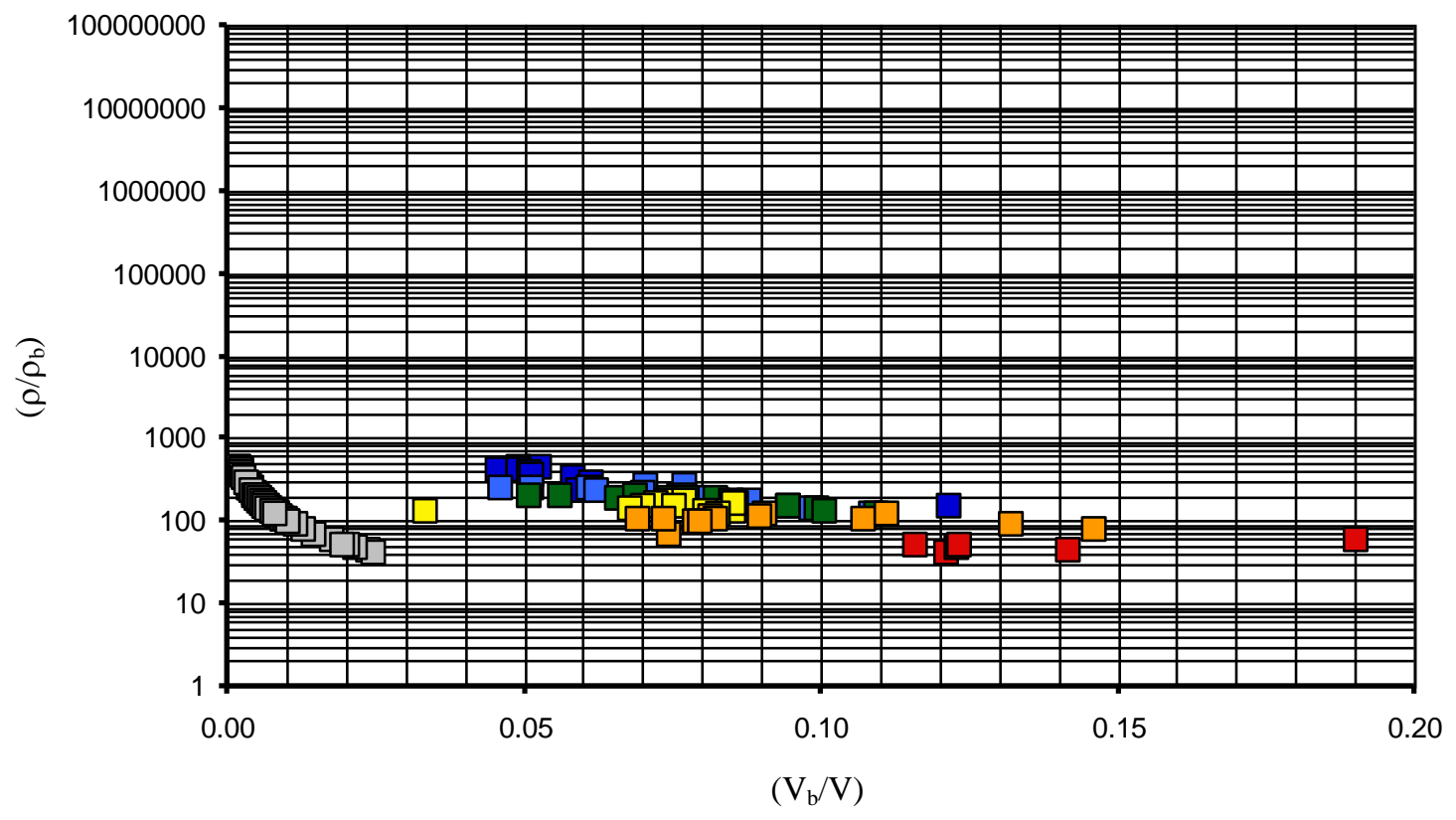

(b) Horizontal resistivity (Arctic data)

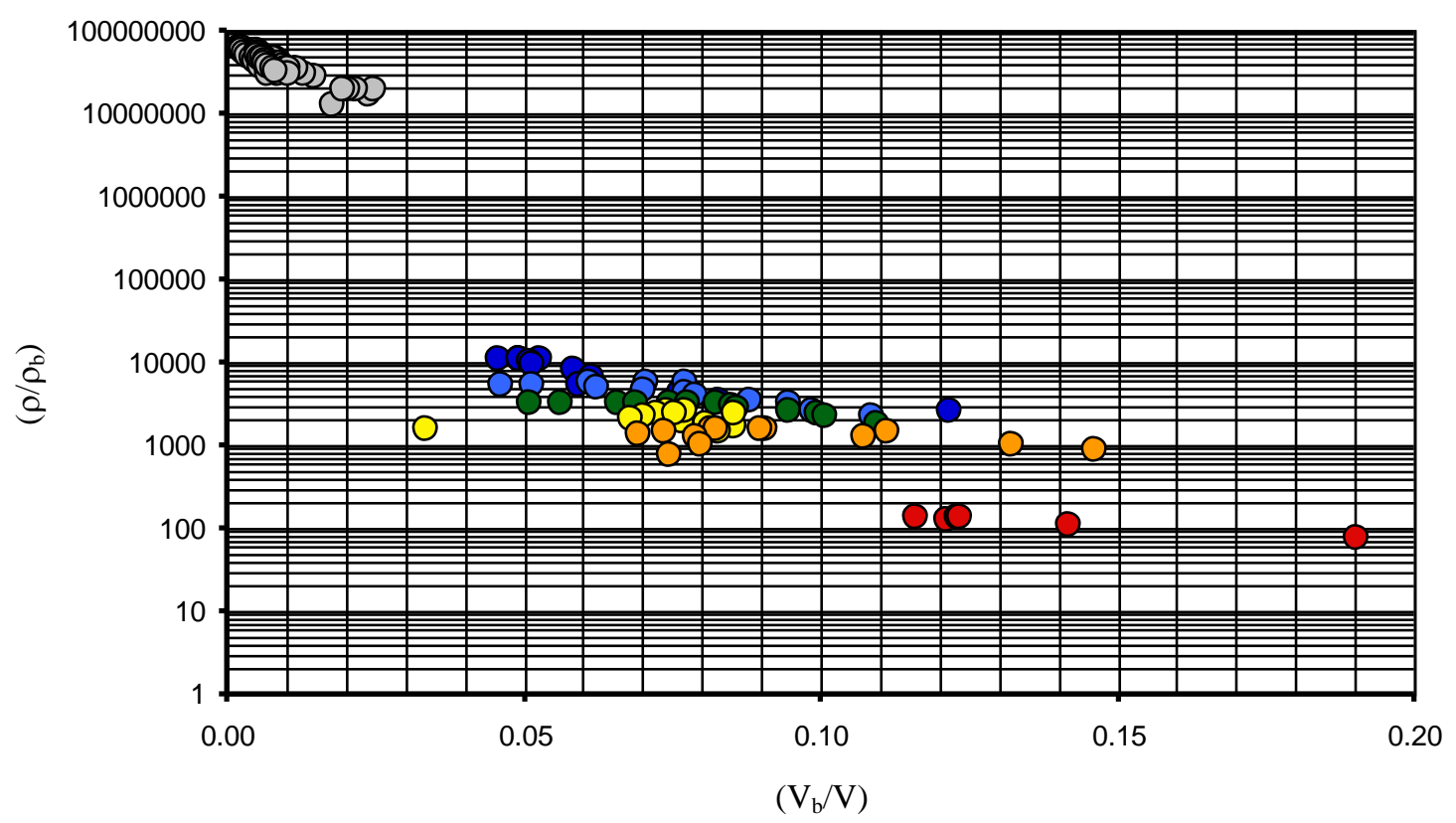

\begin{tabular}{|c|c|c|}
\hline 7-9 Apr & $\square 25-26$ Apr & $\square$ 8-9 May \\
\hline$\square 20-21$ May & $\square$ 28-29 May & $\square 16-17$ Jun \\
\hline$\square$ column stru & & \\
\hline
\end{tabular}

Figure 6.12: Plots of formation factor $\left(\rho / \rho_{b}\right)$ vs. brine volume fraction $\left(V_{b} / V\right)$ of both the proposed column structure (light grey points) and the measured Arctic data (coloured points), for (a) the vertical resistivity and (b) the horizontal resistivity. 
(a) Vertical resistivity (Antarctic data)

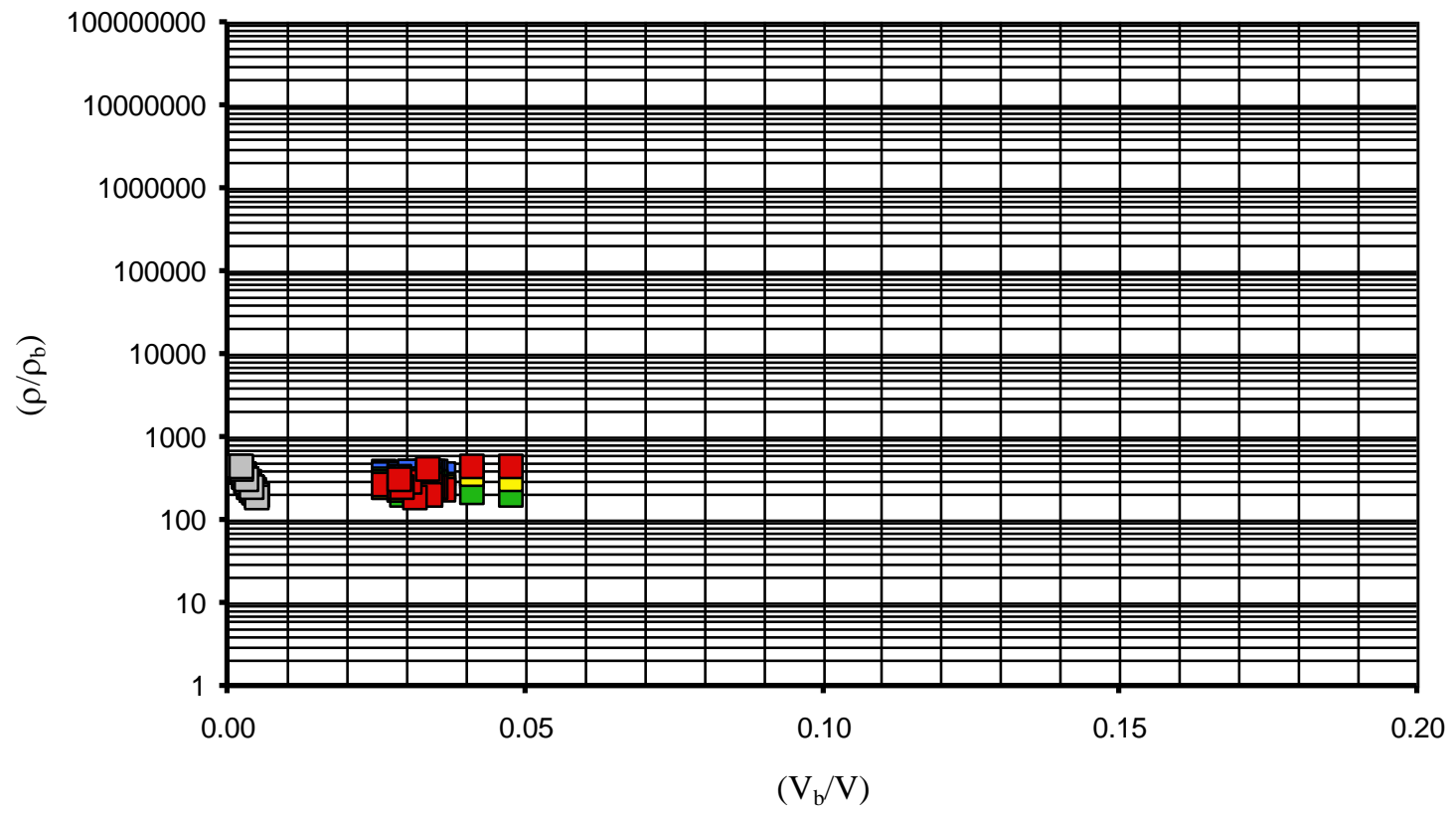

(b) Horizontal resistivity (Antarctic data)

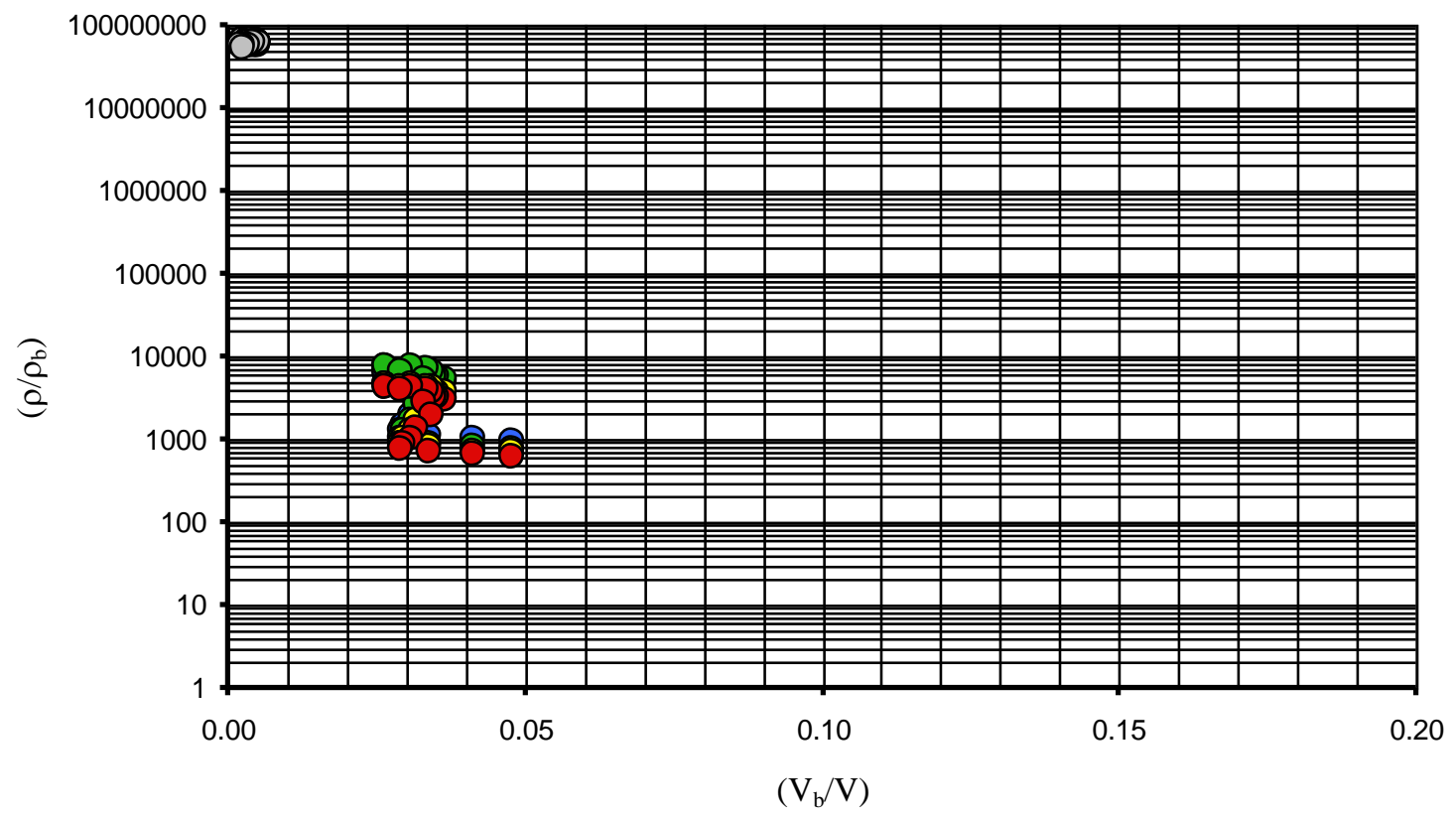

11-12 Nov $\square$ 15-16 Nov $\square$ 19-Nov $\square 21$-Nov $\square$ column structure

Figure 6.13: Plots of formation factor $\left(\rho / \rho_{b}\right)$ vs. brine volume fraction $\left(V_{b} / V\right)$ of both the proposed column structure (light grey points) and the measured Antarctic data (coloured points), for (a) the vertical resistivity and (b) the horizontal resistivity. 
To match the horizontal resistivity we consider a structure comprising of horizontal cubic tubes of a material with a conductivity of $\sigma_{2}$ within a matrix of a second material with a conductivity of $\sigma_{1}$. As we have assumed horizontal isotropy for the sea ice it is necessary to have the same structure in the $x$ and $y$-directions of our model, i.e. tubes in both these directions. A unit cube of this material is shown in Figure 6.14 below.

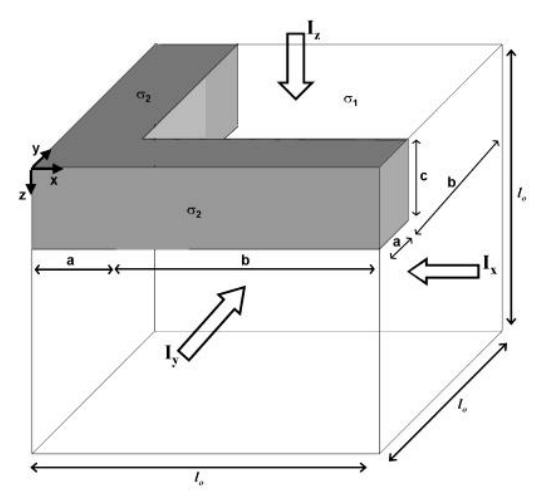

Figure 6.14: Unit cube of a two phase structure, consisting of horizontal cubic tubes of a material with conductivity $\sigma_{2}$, within a matrix of material with conductivity of $\sigma_{1}$. The height and width of the tubes is given by $c$ and $a$ respectively while the distance between parallel tubes is given by $b$. The direction of the $x, y$ and $z$ axes have been labelled.

For this tube structure it can be shown that the horizontal conductivity $\left(\sigma_{H}\right)$ is given by

$$
\sigma_{H}=\frac{(a+b-c)}{(a+b)} \sigma_{1}+\frac{a c}{(a+b)^{2}} \sigma_{2}+\frac{(b c)}{(a+b)} \frac{\sigma_{1} \sigma_{2}}{\left(b \sigma_{2}+a \sigma_{1}\right)}
$$

the vertical conductivity $\left(\sigma_{V}\right)$ is

$$
\sigma_{V}=\frac{b^{2}}{(a+b)^{2}} \sigma_{1}+\frac{\left[(a+b)^{2}-b^{2}\right]}{(a+b)} \frac{\sigma_{1} \sigma_{2}}{\left((a+b-c) \sigma_{2}+c \sigma_{1}\right)}
$$

and the volume fraction of the material with a conductivity of $\sigma_{2}$ is

$$
\frac{V_{2}}{V}=\frac{c\left[(a+b)^{2}-b^{2}\right]}{(a+b)^{3}}
$$

Using the same values for $a$ and $b$ as for the column structure, setting the value for $\sigma_{2}$ by using the brine resistivities calculated from the measured data and setting $\sigma_{l}$ to the DC conductivity of pure ice values of $c$ can be found, using equation (6.16), to closely match the magnitude of the horizontal FF data. Figure 6.15a (Arctic data) and Figure 6.16a (Antarctic data) are plots of horizontal FF vs. brine volume fraction, and they show that the FF (or resistivity) is the same as that obtained from the measured data. 
However, the brine volume fraction is much lower. As with the similar results observed for the column structure and the vertical FF data above this suggests that further brine structure is required.

For the suggested tube structure the vertical FF can be obtained from expression (6.17). From the plots of vertical FF vs. brine volume fraction, as shown in Figure $6.15 b$ (Arctic data) and Figure 6.16b (Antarctic data), it can be seen that for this model, as the suggested tube structure does not have enough vertical connectivity, the vertical FF results are much higher than the observed values. 
(a) Horizontal resistivity (Arctic data)

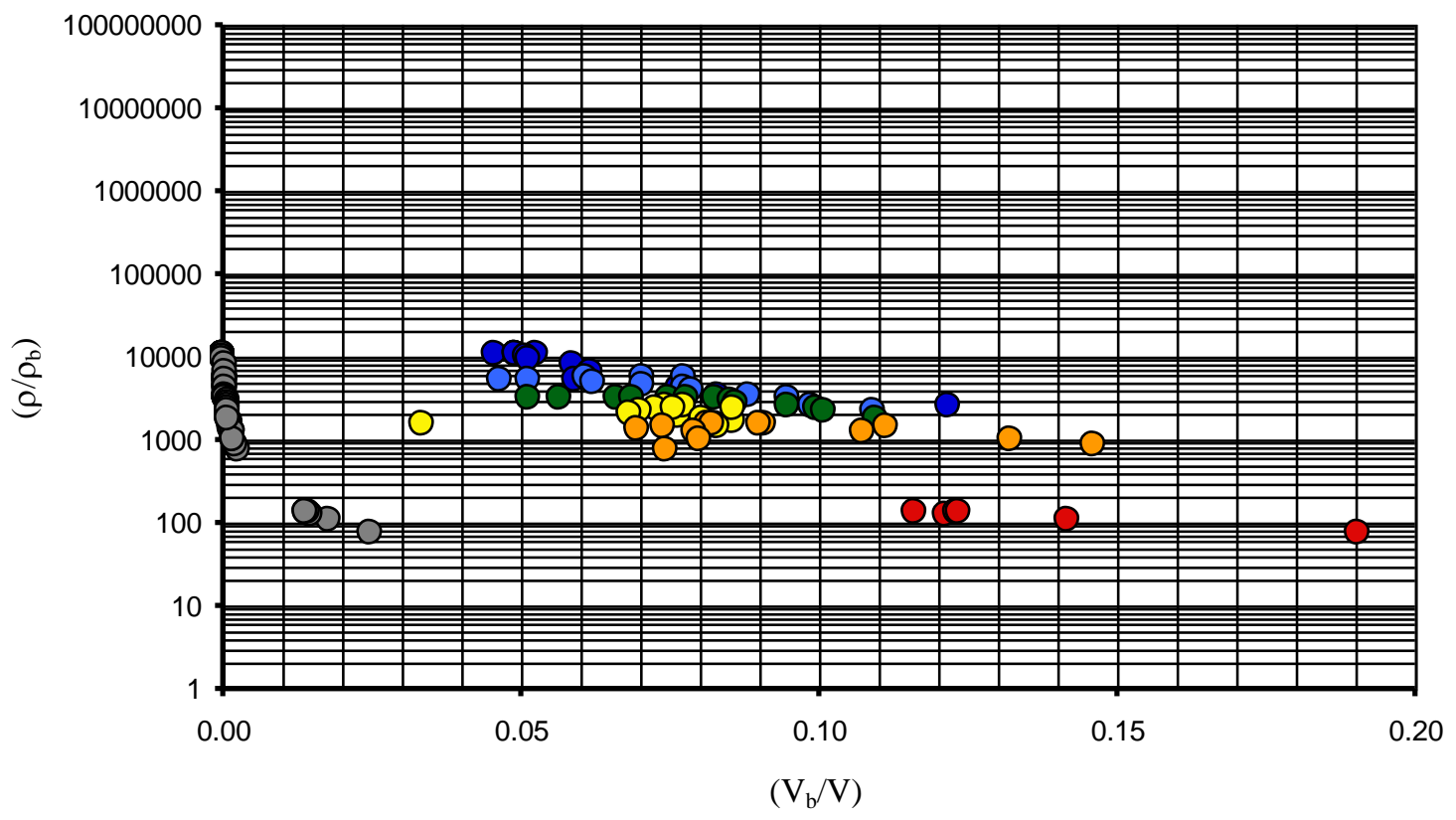

(b) Vertical resistivity (Arctic data)

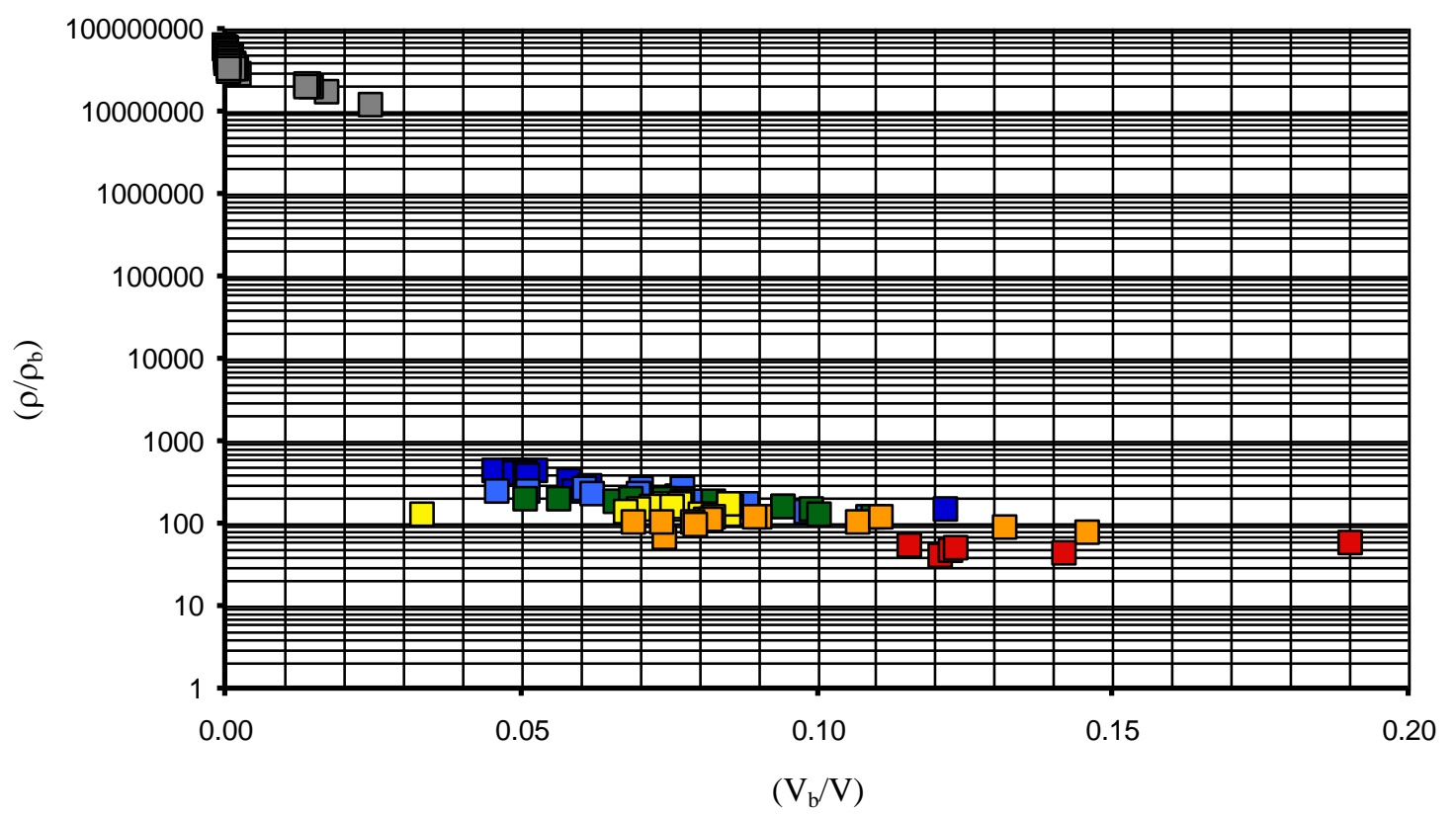

\begin{tabular}{|lll|}
\hline$\square 7-9$ Apr & $\square 25-26$ Apr & $\square 8-9$ May \\
$\square 20-21$ May & $\square 28-29$ May & $\square 16-17$ Jun \\
口tube structure & & \\
\hline
\end{tabular}

Figure 6.15: Plots of formation factor $\left(\rho / \rho_{b}\right)$ vs. brine volume fraction $\left(V_{b} / V\right)$ of both the proposed tube structure (dark grey points) and the measured Arctic data (coloured points), for (a) the horizontal resistivity and (b) the vertical resistivity. 
(a) Horizontal resistivity (Antarctic data)

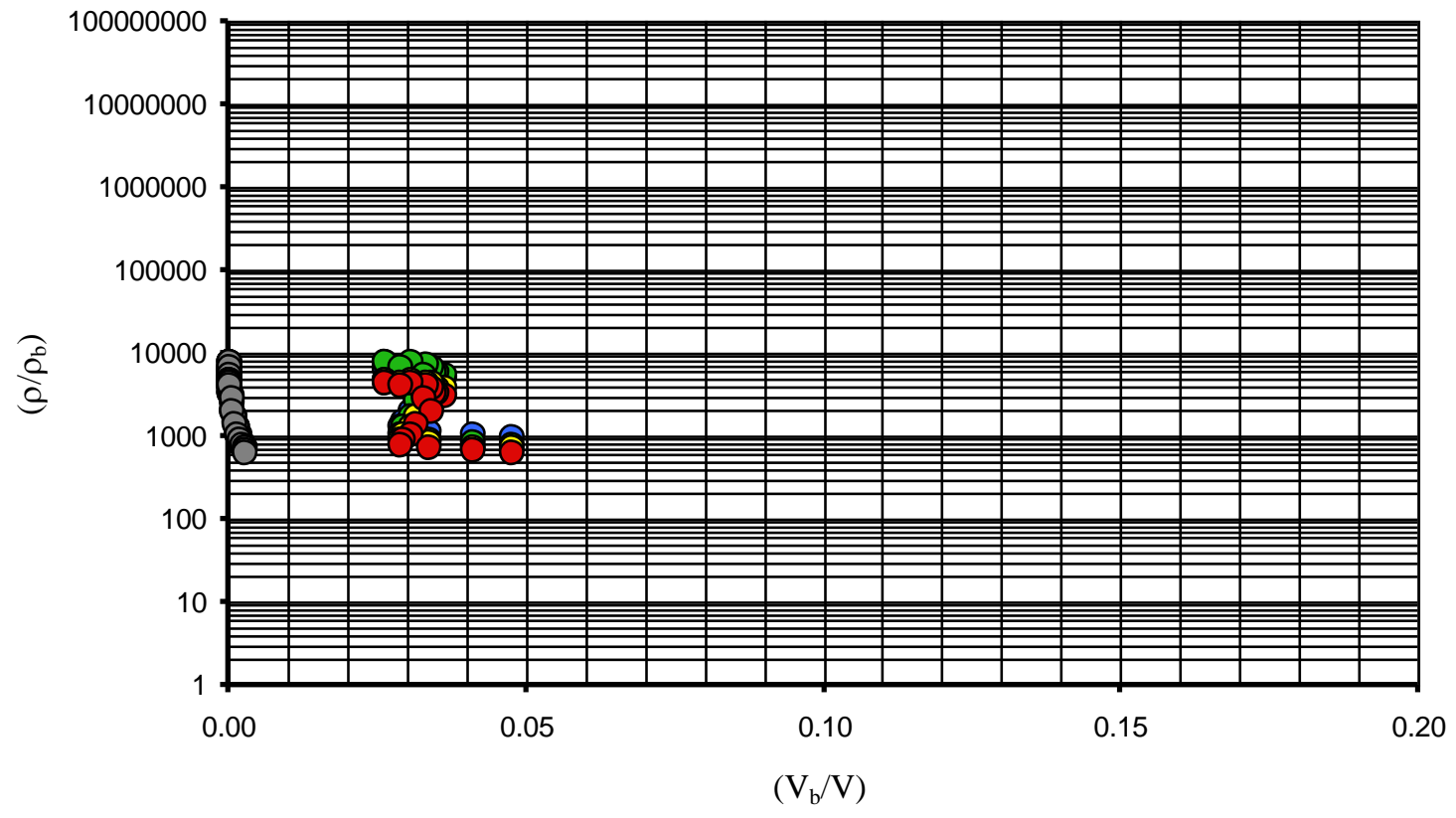

(b) Vertical resistivity (Antarctic data)

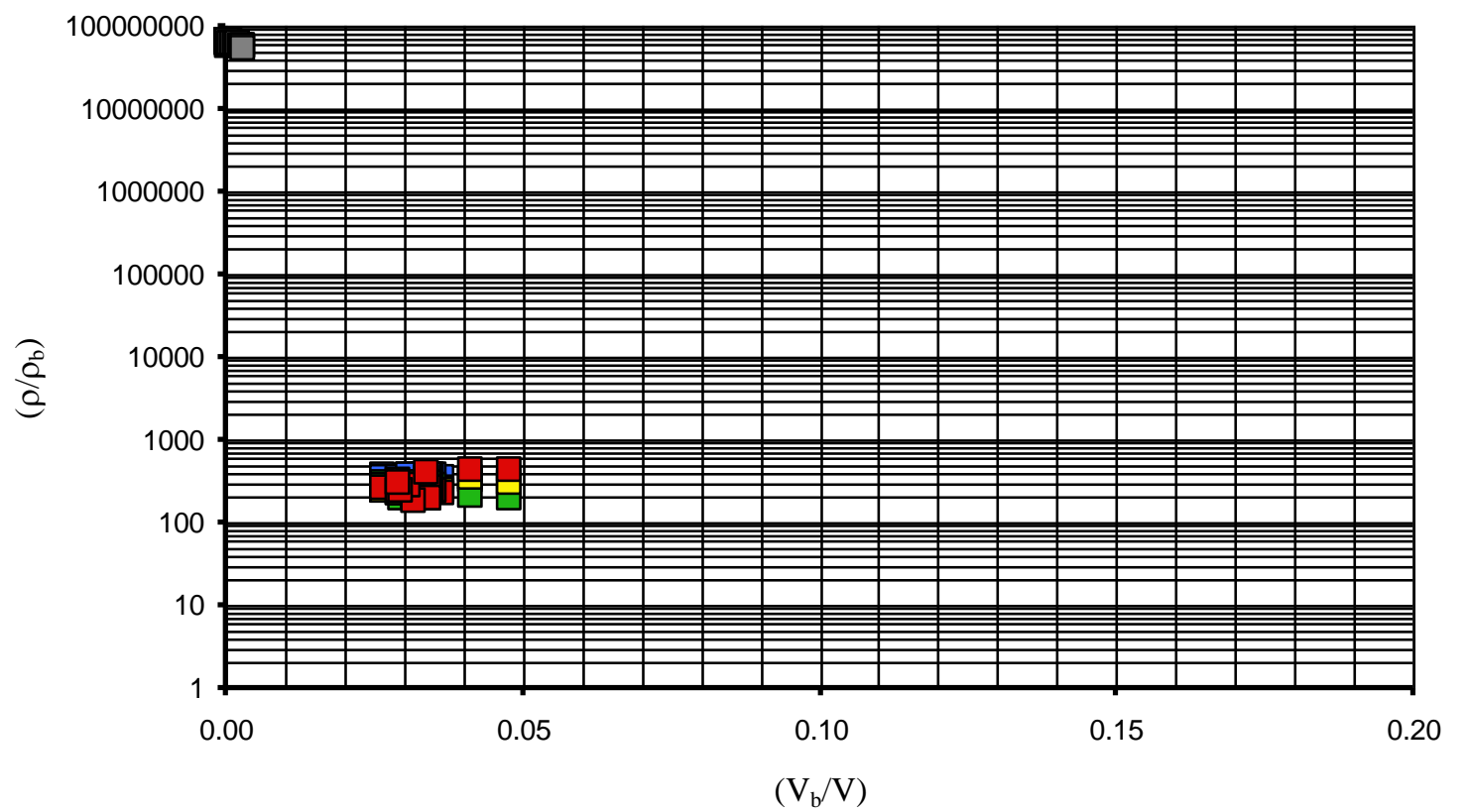

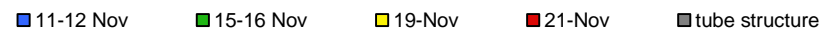

Figure 6.16: Plots of formation factor $\left(\rho / \rho_{b}\right)$ vs. brine volume fraction $\left(V_{b} / V\right)$ of both the proposed tube structure (dark grey points) and the measured Antarctic data (coloured points), for (a) the horizontal resistivity and (b) the vertical resistivity. 
To be able to match both the observed magnitudes of the vertical and horizontal resistivity simultaneously the two previous structures are combined. This creates a structure comprising of vertical cubic columns of a material with a conductivity of $\sigma_{2}$ and horizontal cubic tubes of the same material within a matrix of a second material with a conductivity of $\sigma_{1}$, a unit cube of this material is shown in Figure 6.17 below.

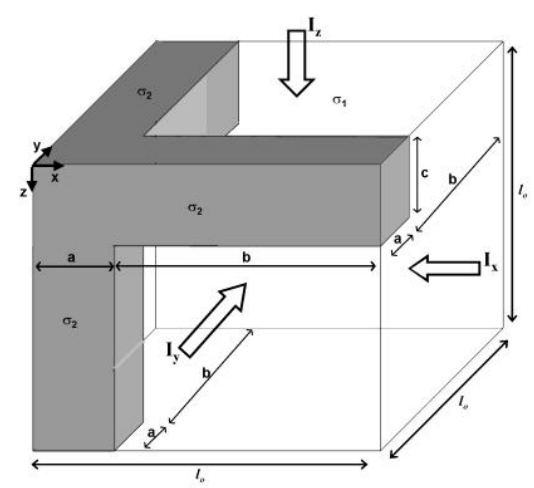

Figure 6.17: Unit cube of a two phase structure, consisting of vertical cubic columns and horizontal cubic tubes of a material with conductivity $\sigma_{2}$, within a matrix of material with conductivity of $\sigma_{1}$. The horizontal distance between the columns and parallel tubes is given by $b$, the horizontal dimensions of the columns and the width of the tubes is given by $a$, and the height of the tubes is given by $c$. The direction of the $x, y$ and $z$ axes have been labelled.

For this combined structure of tubes and columns it can be seen that the horizontal conductivity $\left(\sigma_{H}\right)$ is given by

$$
\begin{aligned}
\sigma_{H}=\frac{b(a+b-c)}{(a+b)^{2}} \sigma_{1}+\frac{a c}{(a+b)^{2}} \sigma_{2} \\
+\frac{a(a+b-c)+b c}{(a+b)} \frac{\sigma_{1} \sigma_{2}}{b \sigma_{2}+a \sigma_{1}}
\end{aligned}
$$

while the vertical conductivity $\left(\sigma_{V}\right)$ is

$$
\sigma_{V}=\frac{b^{2}}{(a+b)^{2}} \sigma_{1}+\frac{a^{2}}{(a+b)^{2}} \sigma_{2}+\frac{2 a b}{(a+b)} \frac{\sigma_{1} \sigma_{2}}{(a+b-c) \sigma_{2}+c \sigma_{1}}
$$

Furthermore, the volume fraction of the material with a conductivity of $\sigma_{2}$ is given by

$$
\frac{V_{2}}{V}=\frac{2 a b c+a^{2}(a+b)}{(a+b)^{3}}
$$

Using the same values for $a, b$ and $c$ as found in the independent tube and column structures (for both the Arctic and Antarctic data), once again setting $\sigma_{2}$ by using brine resistivities calculated from the measured data and setting $\sigma_{l}$ to the DC conductivity of 
pure ice, equation (6.19) and (6.20) can be used to find the vertical and horizontal FFs. Plots of vertical and horizontal FFs vs. brine volume fraction, displaying the Arctic data, are shown in Figure 6.18a and Figure 6.18b respectively. The Antarctic data are included in Figure 6.19a and Figure 6.19b which display the vertical and horizontal FFs vs $V_{b} / V$ respectively. From these graphs it can be seen that the FFs (or resistivities) closely match those obtained from the measured data, however, the brine volume fraction is still much lower. Thus the structure shown in Figure 6.17 gives both the required vertical and horizontal resistivities for the Arctic and Antarctic data, but some additional brine structure is still required. 
(a) Vertical resistivity (Arctic data)

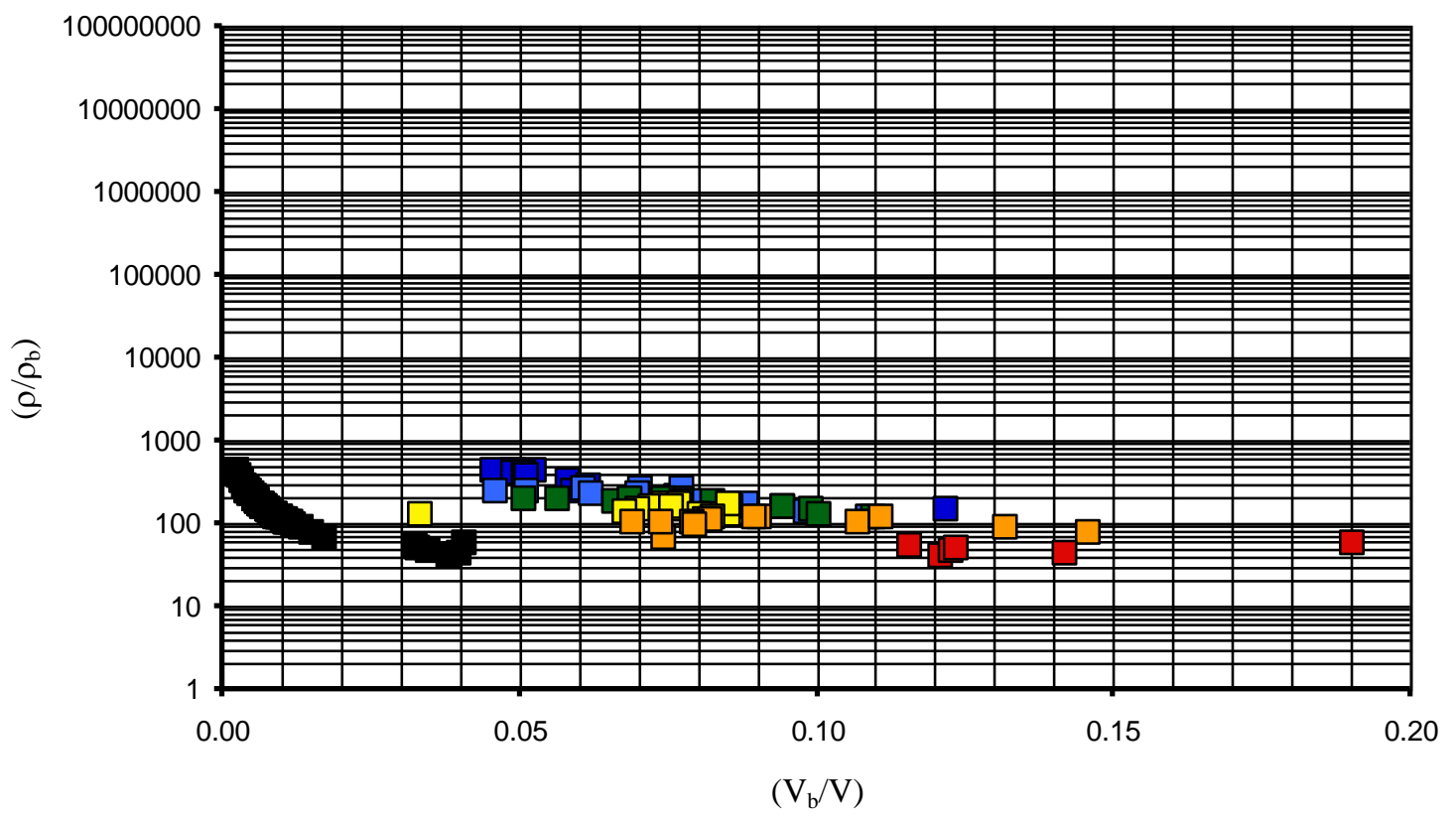

(b) Horizontal resistivity (Arctic data)

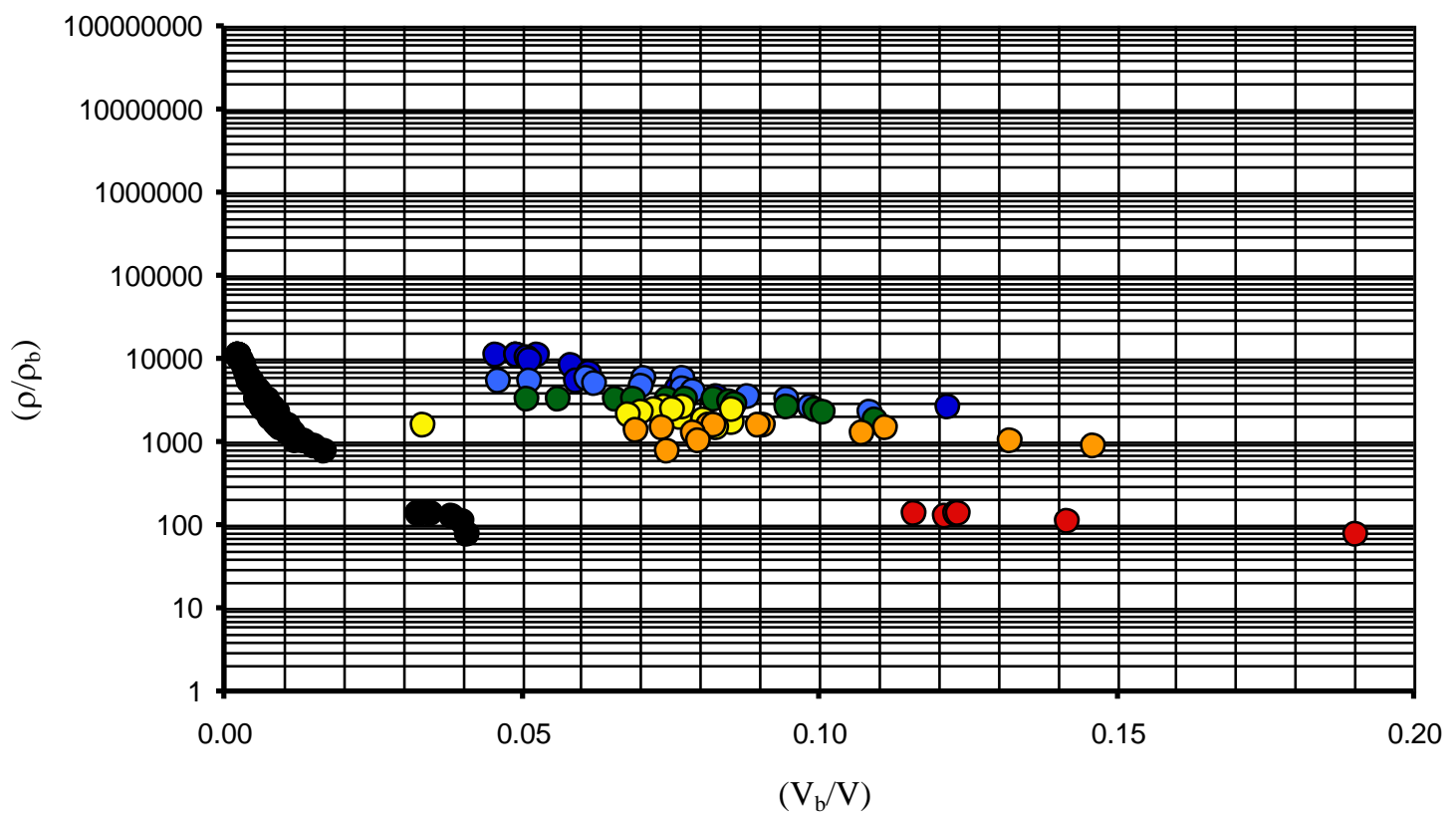

\begin{tabular}{|lll|}
\hline$\square 7-9 \mathrm{Apr}$ & $\square 25-26 \mathrm{Apr}$ & $\square 8-9$ May \\
$\square 20-21$ May & $\square 28-29$ May & $\square 16-17 \mathrm{Jun}$ \\
—column and tube structure & & \\
\hline
\end{tabular}

Figure 6.18: Plots of formation factor $\left(\rho / \rho_{b}\right)$ vs. brine volume fraction $\left(V_{b} / V\right)$ of both the proposed column and tube structure (black points) and the measured Arctic data (coloured points), for (a) the vertical resistivity and (b) the horizontal resistivity. 
(a) Vertical resistivity (Antarctic data)

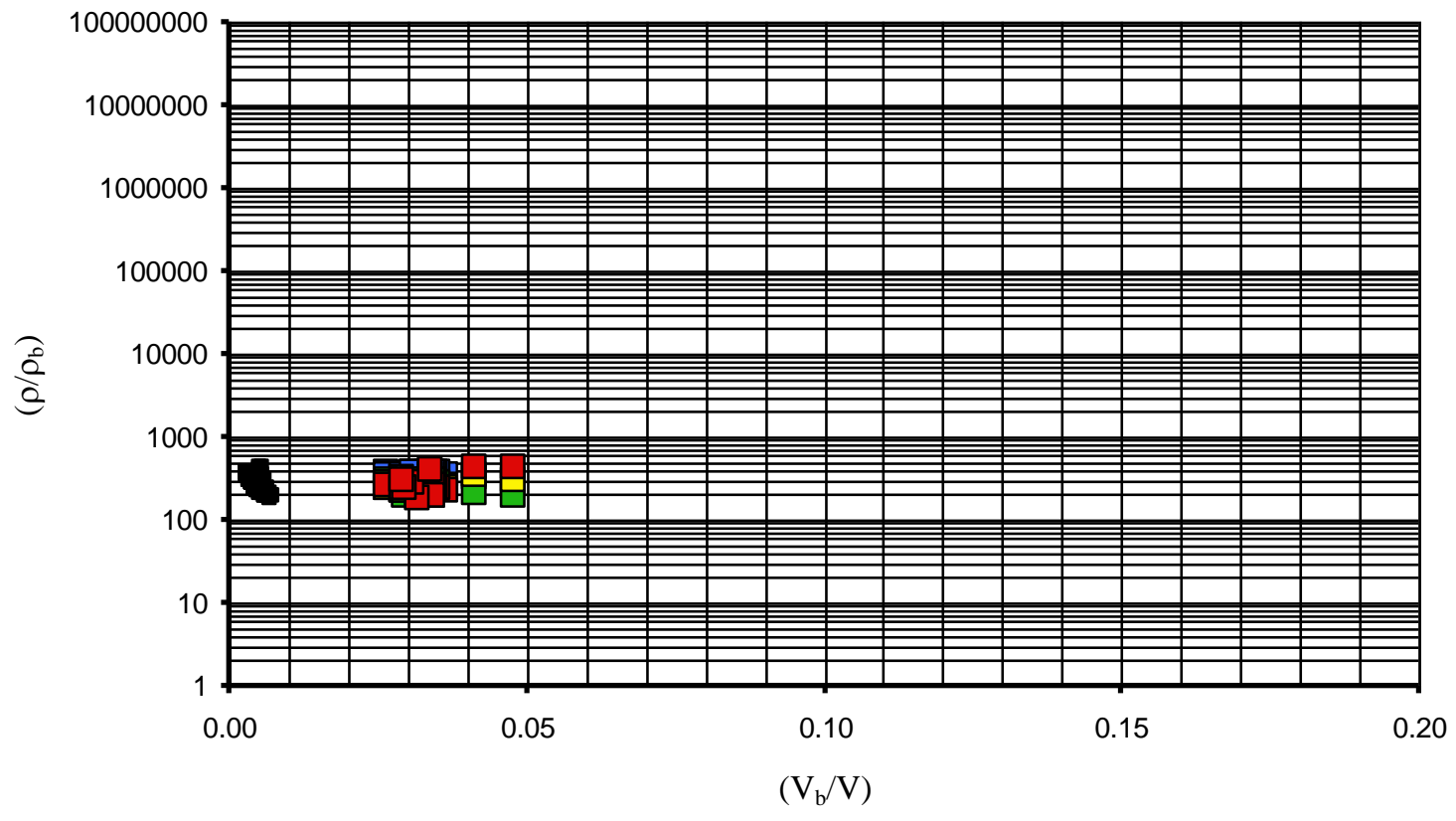

(b) Horizontal resistivity (Antarctic data)

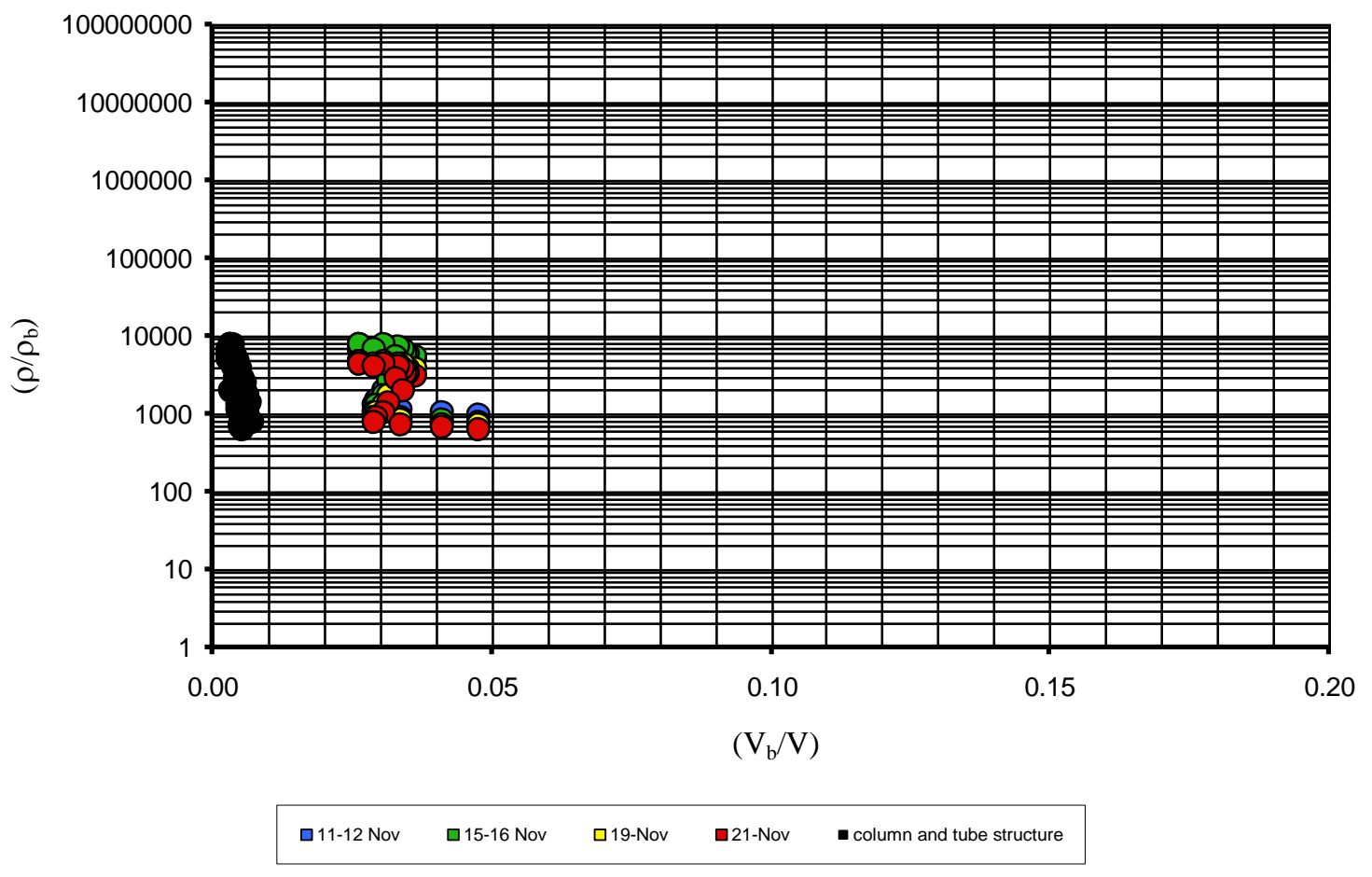

Figure 6.19: Plots of formation factor $\left(\rho / \rho_{b}\right)$ vs. brine volume fraction $\left(V_{b} / V\right)$ of both the proposed column and tube structure (black points) and the measured Antarctic data (coloured points), for (a) the vertical resistivity and (b) the horizontal resistivity. 
One way to increase the brine volume fraction without significantly affecting the vertical or horizontal resistivities is to introduce isolated cubes of brine of dimension $d$ as illustrated in Figure 6.20, below. The resistivity of this new structure (tubes, cubes and columns of brine) can be shown to be given by the following expressions

$$
\begin{aligned}
& \sigma_{H}=\frac{\left(b(a+b-c)-d^{2}\right)}{(a+b)^{2}} \sigma_{1}+\frac{a c}{(a+b)^{2}} \sigma_{2} \\
&+\frac{(b c+a(a+b-c))}{(a+b)} \frac{\sigma_{1} \sigma_{2}}{b \sigma_{2}+a \sigma_{1}} \\
&+\frac{d^{2}}{(a+b)} \frac{\sigma_{1} \sigma_{2}}{(a+b-d) \sigma_{2}+d \sigma_{1}}
\end{aligned}
$$

and

$$
\begin{gathered}
\sigma_{v}=\frac{\left(b^{2}-d^{2}\right)}{(a+b)^{2}} \sigma_{1}+\frac{a^{2}}{(a+b)^{2}} \sigma_{2}+\frac{2 a b}{(a+b)} \frac{\sigma_{1} \sigma_{2}}{(a+b-c) \sigma_{2}+c \sigma_{1}} \\
+\frac{d^{2}}{(a+b)} \frac{\sigma_{1} \sigma_{2}}{(a+b-d) \sigma_{2}+d \sigma_{1}}
\end{gathered}
$$

while the brine volume fraction is given by

$$
\frac{V_{b}}{V}=\frac{2 a b c+a^{2}(a+b)+d^{3}}{(a+b)^{3}}
$$

Note that the above expressions hold for any position of the cube of brine within the $b$ by $b$ by $a+b-c$ area of pure ice left free by the column and tube structures. In Figure 6.20 below the cube is arbitrarily shown in the far corner of this region of the solid ice matrix.

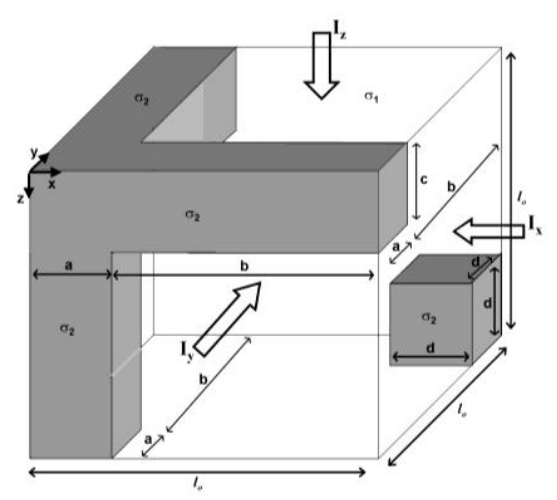

Figure 6.20: Unit cube of a two phase structure, consisting of vertical cubic columns, horizontal cubic tubes and cubes of a material with conductivity $\sigma_{2}$, within a matrix of material with conductivity of $\sigma_{1}$. The horizontal distance between the columns and parallel tubes is given by $b$, the horizontal dimensions of the columns and the width of the tubes is given by $a$, the height of the tubes is given by $c$, and the dimensions of the cubes are given by $d$. The direction of the $x, y$ and $z$ axes have been labelled. 
Knowing the difference between the brine volume fractions obtained from the measurement data and the suggested column and tube structure the required dimensions (d) of the cube can be found by equating this difference to the volume fraction of the cube giving

$$
\Delta \frac{V_{b}}{V}=\frac{d^{3}}{(a+b)^{3}} \Rightarrow d=\sqrt[3]{\left(\Delta \frac{V_{b}}{V}\right)(a+b)^{3}}
$$

Using the same values for $a, b$ and $c$ as found in the independent tube and column structures, the value of $d$ obtained from the expression above, using the brine resistivities calculated from the measured data to set $\sigma_{2}$ and setting $\sigma_{1}$ to the DC conductivity of pure ice equation (6.22) and (6.23) can be used to find the vertical and horizontal FFs for both the Arctic and Antarctic data. In Figure 6.21 and Figure 6.22 below it can be seen that a structure comprised of columns, tubes and isolated cubes of brine can be constructed so that the FF's and brine volume fractions of the structure closely match those obtained from the measured data (differences between the values obtained from the proposed structures and the values obtained from the measured data result in errors of less than $1 \%$ ). 
(a) Vertical resistivity (Arctic data)

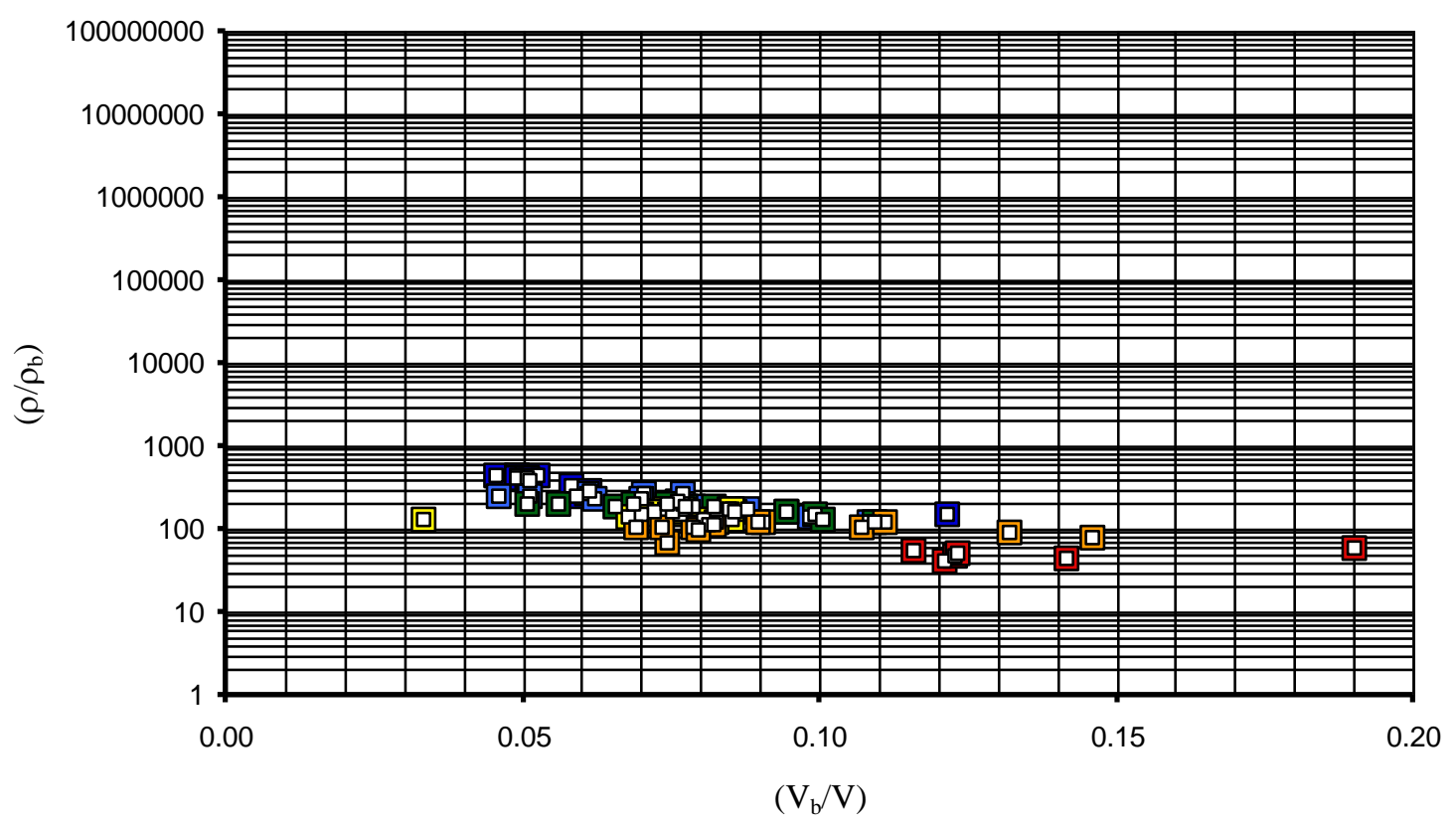

(b) Horizontal resistivity (Arctic data)
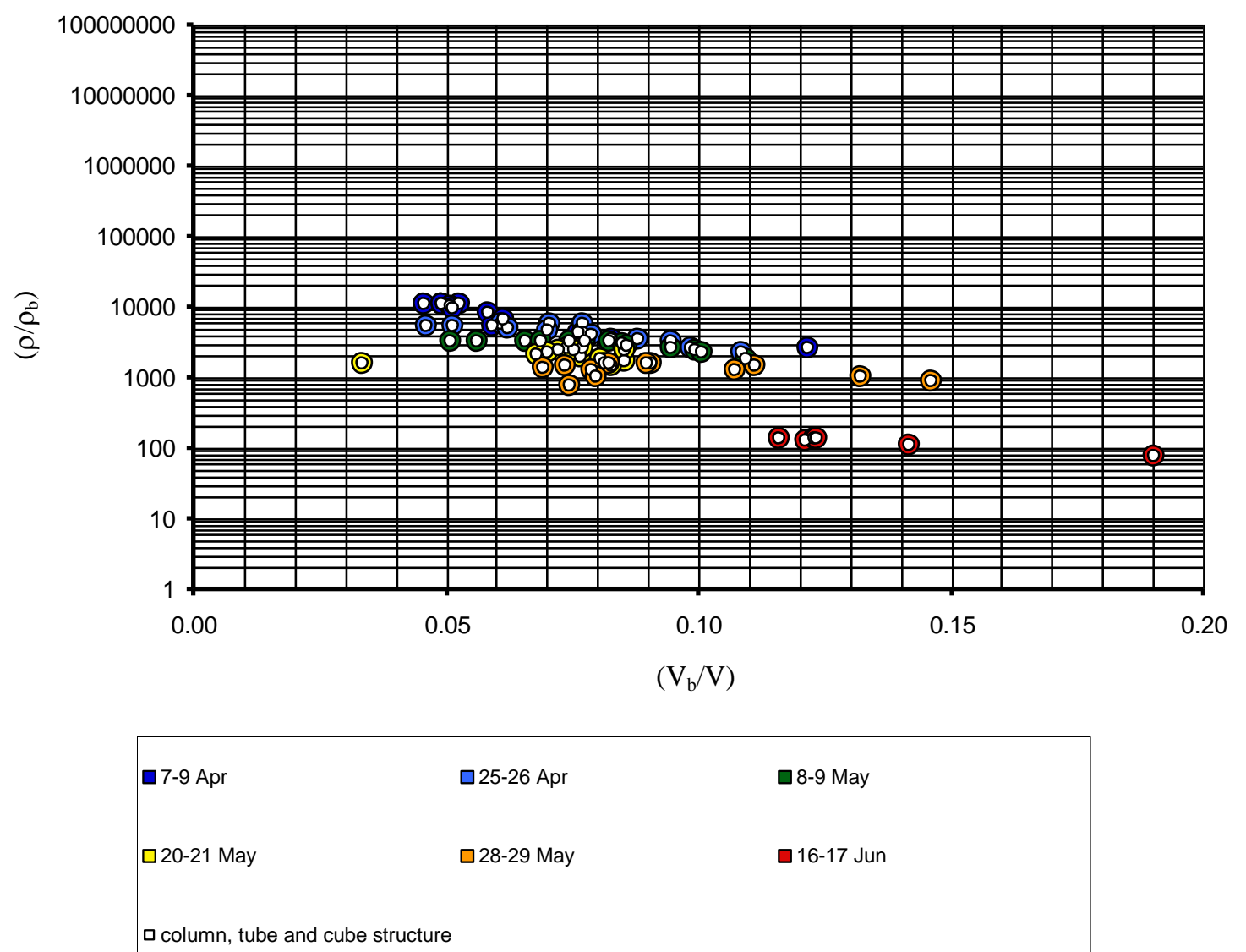

Figure 6.21: Plots of formation factor $\left(\rho / \rho_{b}\right)$ vs. brine volume fraction $\left(\mathrm{V}_{\mathrm{b}} / \mathrm{V}\right)$ of both the proposed column, tube and cube structure (white points) and the measured Arctic data (coloured points), for (a) the vertical resistivity and (b) the horizontal resistivity. 
(a) Vertical resistivity (Antarctic data)

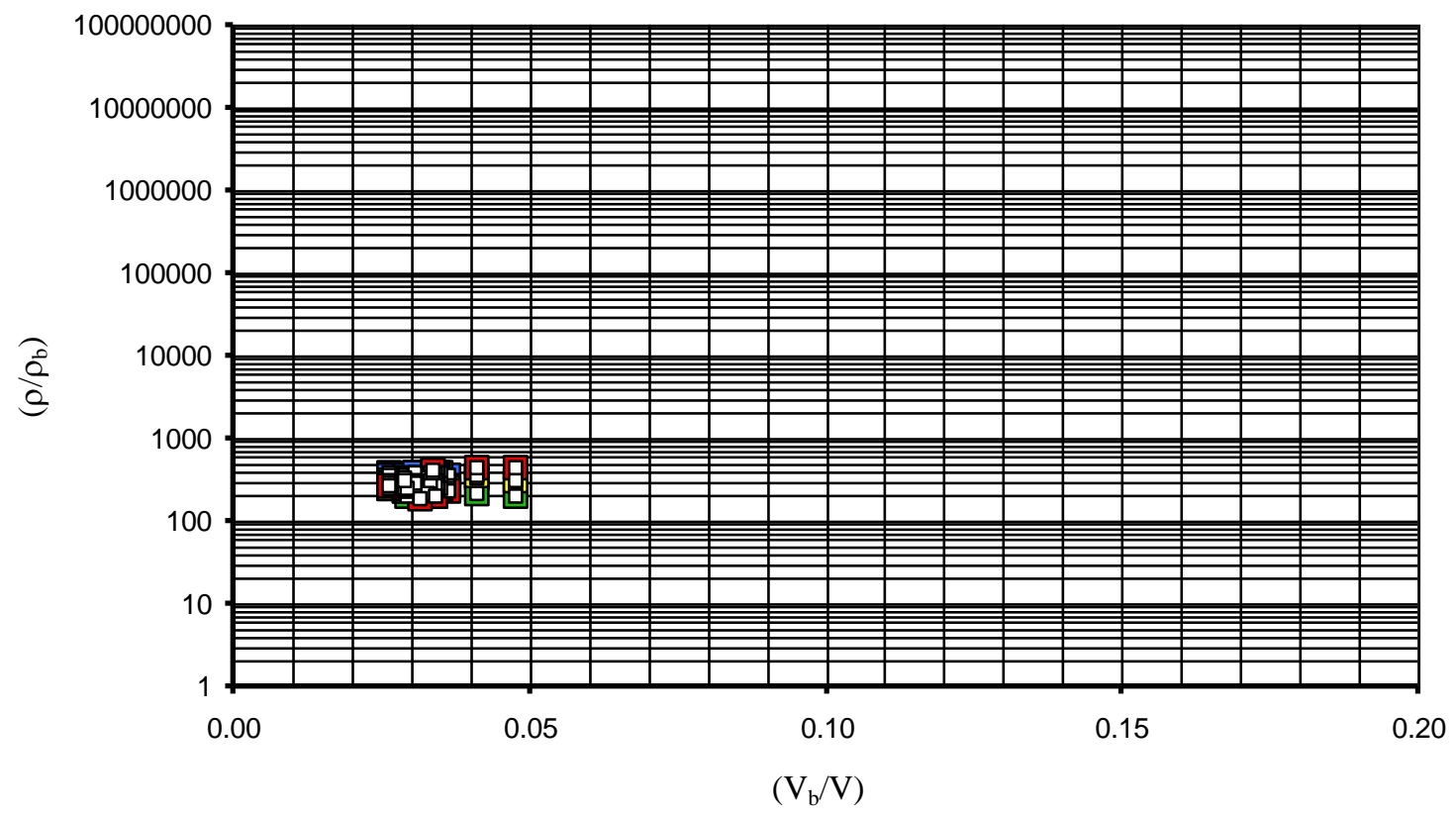

(b) Horizontal resistivity (Antarctic data)

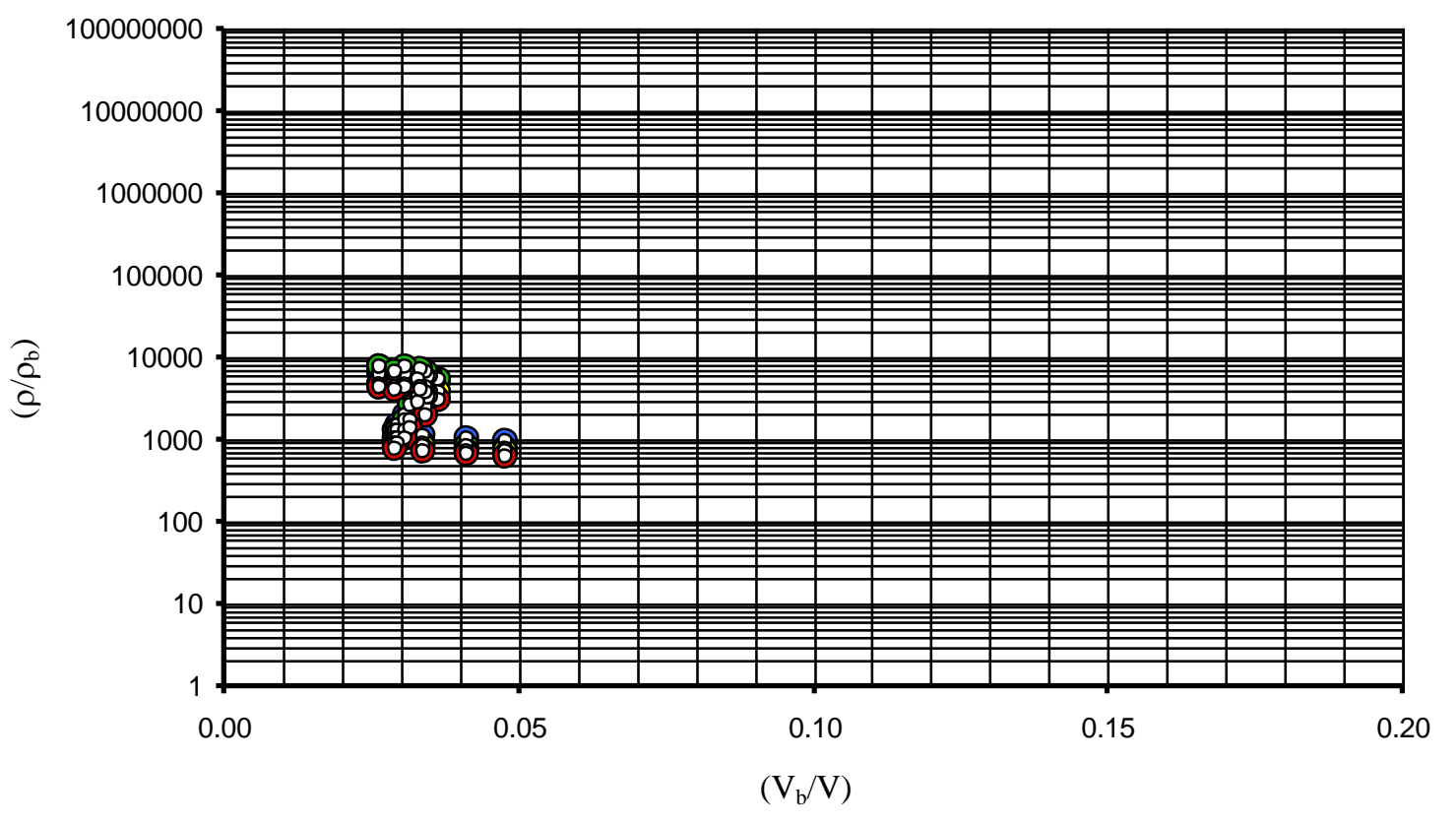

\begin{tabular}{|c|c|c|c|c|}
\hline$\square 11-12$ Nov & 口15-16 Nov & 口19-Nov & 口21-Nov & $\square$ column, tube and cube structure \\
\hline
\end{tabular}

Figure 6.22: Plots of formation factor $\left(\rho / \rho_{b}\right)$ vs. brine volume fraction $\left(V_{b} / V\right)$ of both the proposed column, tube and cube structure (white points) and the measured Antarctic data (coloured points), for (a) the vertical resistivity and (b) the horizontal resistivity. 
The data can therefore be well fit by this structure given the brine resistivities calculated from temperature data and an estimate at the conductivity of pure ice $\left(\sigma_{l}=0.0000001 \mathrm{Sm}^{-1}\right.$ as given by Mathews and Clark (1963)). However, as previously discussed the conductivity of the solid ice matrix is not well constrained. Thus it is important to consider how changing the conductivity of the ice $\left(\sigma_{l}\right)$ affects the resistivity values obtained from the model structure.

By taking average values for the conductivity of the brine and the dimensions of the structure ( $a, b, c$, and $d$ ) equations (6.22) and (6.23) can be used to find the horizontal and vertical conductivity of the structure for different values of the conductivity of the ice matrix. Figure 6.23 (Arctic data) and Figure 6.24 (Antarctic data) below, show plots of conductivity of the model structure vs. differing conductivities of the ice matrix. From these plots it can be seen that for both the Arctic and Antarctic data the column, tube and cube structure shows horizontal (blue curve) and vertical (green curve) conductivities that do not change significantly with increasing ice conductivity until that conductivity reaches $0.001 \mathrm{Sm}^{-1}$ (corresponds to a resistivity of $1000 \Omega \mathrm{m}$ ). Suggesting that the solid ice matrix has a resistivity of less than $1000 \Omega \mathrm{m}$ seems unrealistic, as most of the salts are rejected from the ice lattice, forming brine inclusions, as the seawater freezes. Thus the suggested models appear robust to changes of the conductivity of the ice, within a sensible range.

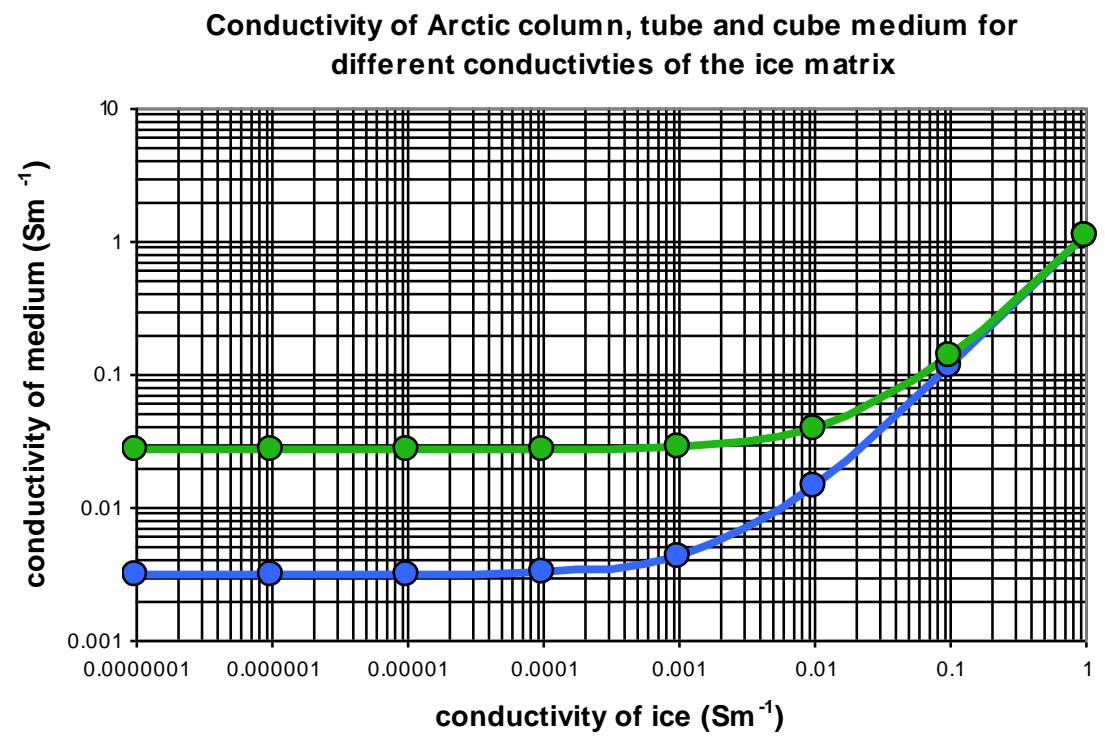

Figure 6.23: Plot of the conductivity of the proposed Arctic column, tube and cube structure for differing conductivities of the ice matrix. The blue curve shows the trend of the horizontal component of the bulk conductivity, while the green curve shows the trend of the vertical component of the bulk conductivity. 


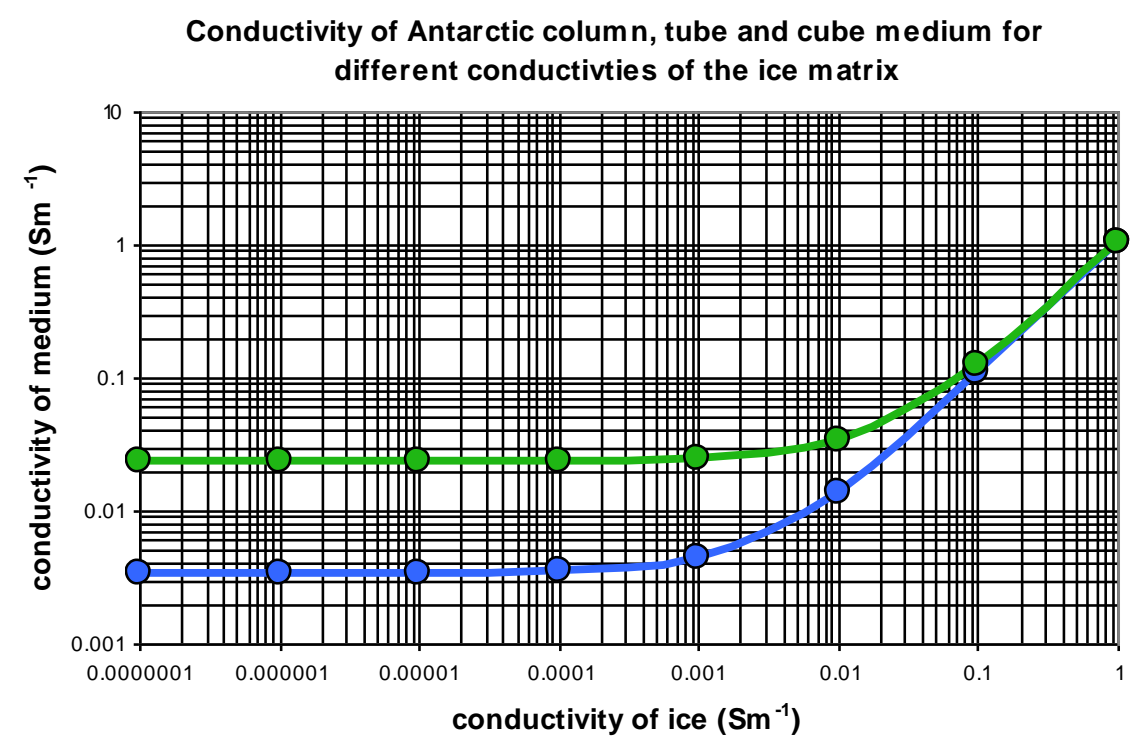

Figure 6.24: Plot of the conductivity of the proposed Antarctic column, tube and cube structure for differing conductivities of the ice matrix. The blue curve shows the trend of the horizontal component of the bulk conductivity, while the green curve shows the trend of the vertical component of the bulk conductivity.

The proposed dimensions of the columns, tubes and cubes are not fixed values, rather it is the relation between the dimensions that must remain constant. This means that if all dimensions are multiplied by an arbitrary value the conductivity of the model structure will remain the same. Despite this the structure shown in Figure 6.20 still indicates that there must be horizontal and vertical connectivity of brine to obtain the resistivities measured, and additional isolated brine pockets to give the observed brine volume fraction. The relative sizes are quite well constrained, with an increase of $1 \%$ in any one of the dimensions leading to a misfit to the data of approximately the same size, while an increase of $5 \%$ produces a misfit of $5 \%$ to $10 \%$.

One way of allowing the proposed structure to be physically realistic (in terms of dimension) is to initially set one of the dimensions to a value suggested by the literature and then allow the dimensions to evolve around this to give the required resistivities. The dimensions of the proposed structure are shown in Figure 6.25 (Arctic model) and Figure 6.26 (Antarctic model) below, where the dimensions are expressed as multiples of the initial dimension of the columns (i.e. that from the surface of the ice on the 7-9 April 2008 and the 11-12 Nov 2009). 
(a) Horizontal dimensions of columns (a)

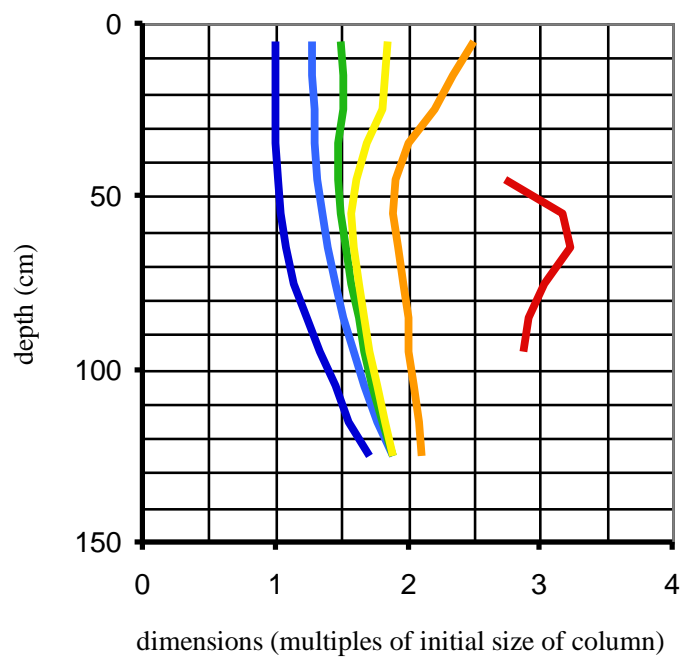

(c) Thickness of horizontal tubes (c)

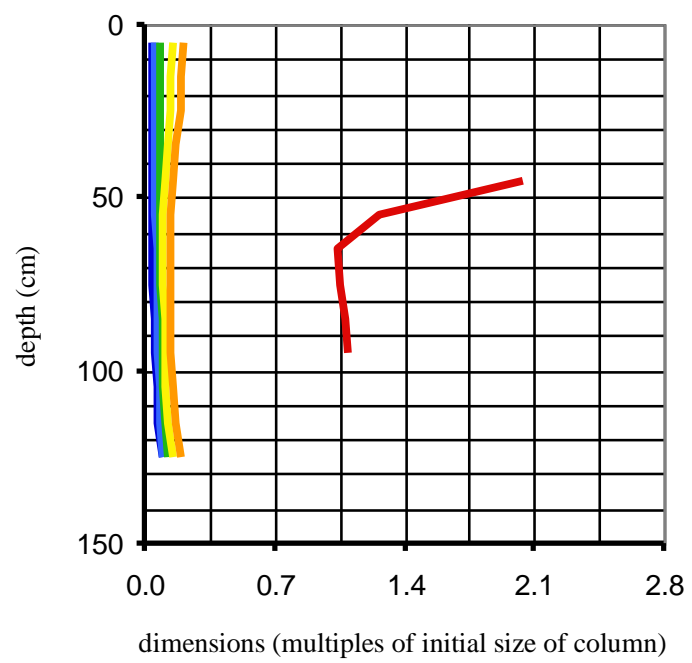

(b) Spacing between columns $(b)$

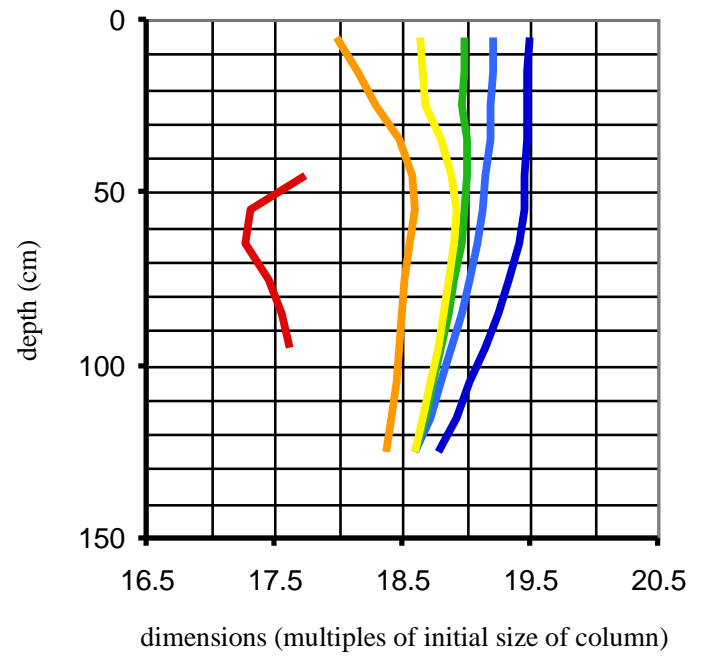

(d) Dimensions of cubes $(d)$

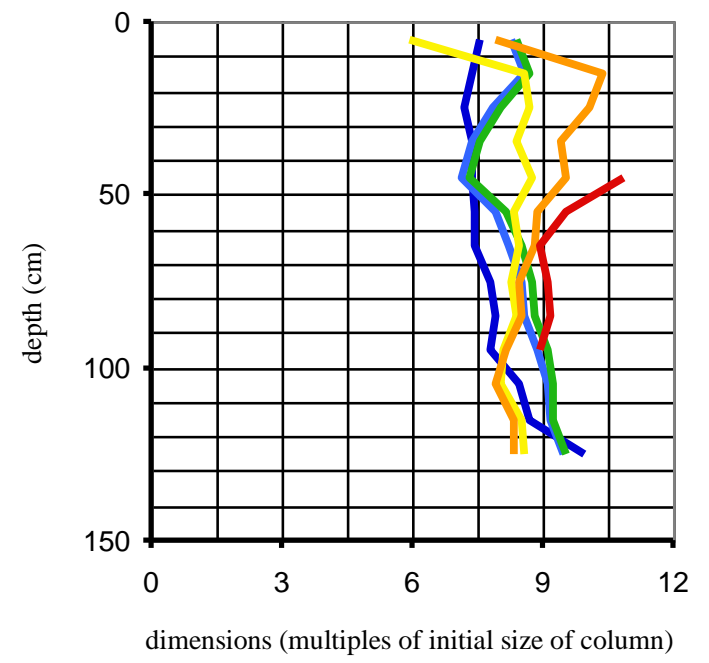

\begin{tabular}{|lll|}
\hline 7-9 Apr & 25-26 Apr & -8 -9 May \\
20-21 May & 28-29 May & $-16-17$ Jun \\
\hline
\end{tabular}

Figure 6.25: Plots of the relative sizes, and the trends with time and depth, of the dimensions of the proposed Arctic brine structure. (a) the horizontal dimensions of the columns $(a)$, (b) the spacing between the columns $(b)$, (c) the thickness of the horizontal tubes $(c)$ and (d) the dimensions of the cubes $(d)$. 
(a) Horizontal dimensions of columns (a)

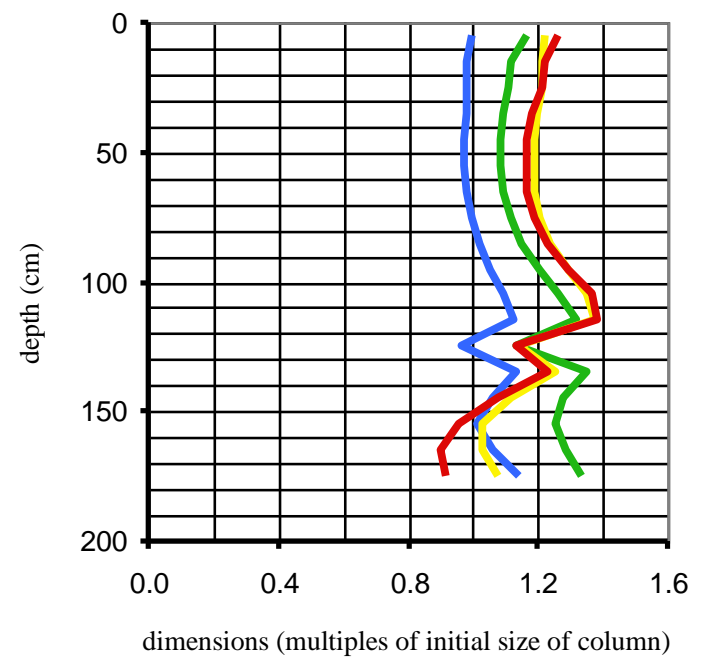

(c) Thickness of horizontal tubes (c)

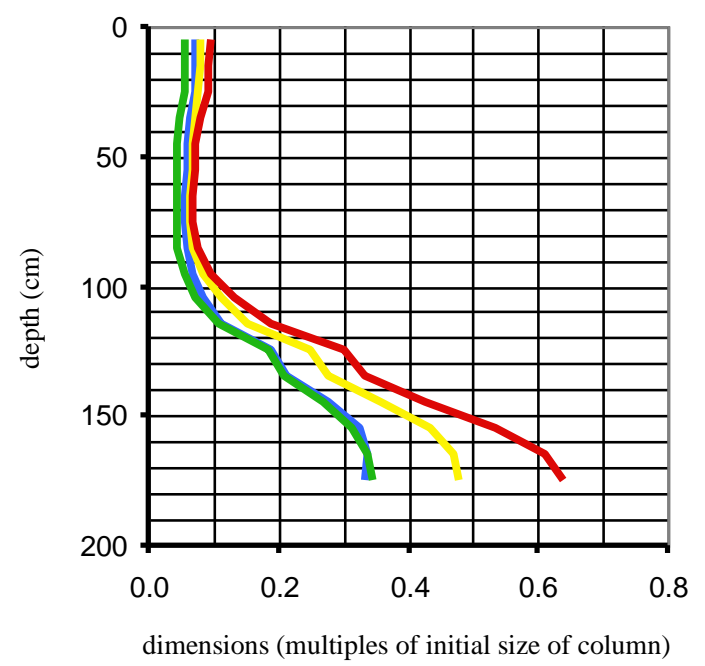

(b) Spacing between columns (b)

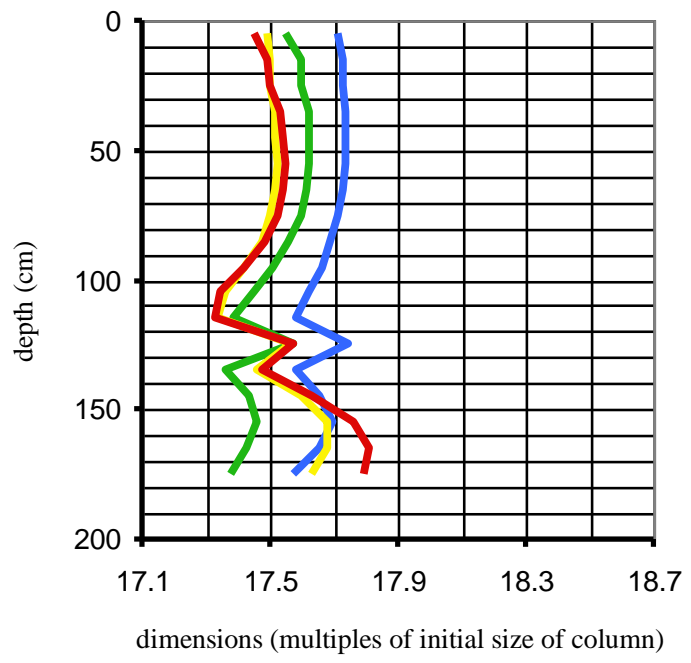

(d) Dimensions of cubes $(d)$

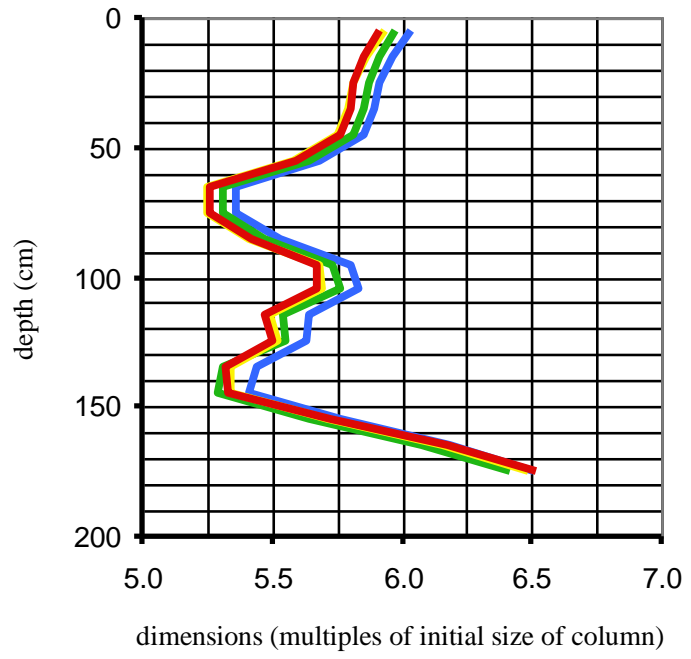

-11-12 Nov $-15-16 \mathrm{Nov}$ Nov $21 \mathrm{Nov}$

Figure 6.26: Plots of the relative sizes, and the trends with time and depth, of the dimensions of the proposed Antarctic brine structure. (a) the horizontal dimensions of the columns (a), (b) the spacing between the columns $(b)$, (c) the thickness of the horizontal tubes $(c)$ and (d) the dimensions of the cubes (d). 
For the brine structure model created for the Arctic data (Figure 6.25) the horizontal dimensions $(a)$ of the columns increase over the measurement period as the ice warms up. Additionally, for most of the measurement periods, the column dimensions are greater at the bottom and, for later measurements, also at the top of the ice, consistent with the ice being warmer in these regions. Only the model for the 16-17 June differs from this with apparently thinner columns at the surface and base of the ice. It should be noted that the 16-17 June profile is more compact than the other profiles due to surface and bottom melting which occurs on the ice cover. Additionally high surface resistivities, probably caused by flushing of brine by melt water, have been removed. Despite this it would seem that the decreasing salinity of the brine seen as the ice melts may be affecting the model brine structure obtained for this late measurement period. The separation between the columns $(b)$ is, as expected, the mirror image of the size of the columns.

The thickness of the horizontal tubes $(c)$ also displays an increase in size over the measurement period with a large jump between the 28-29 May and 16-17 June models. For the $c$ dimension of the tubes all models display a similar depth variation to that seen with the $a$ dimension of the columns, with slightly larger dimensions at the base and later also at the surface of the ice. The dimensions of the isolated cubes $(d)$ show significant overlapping of model values and no clear trends, however, there is a general increasing in dimension as temperatures increase.

For the Antarctic data the brine structure (Figure 6.26) shows no clear trend over the measurement period with the values for the models of the different dates more closely grouped. This probably reflects the small variation in the temperature profiles of the ice over the four measurement sets. Having said this there is a slight increasing trend in the dimensions of the brine inclusions as the temperature increases, this is perhaps most discernible in the thickness of the horizontal tubes $(c)$.

The horizontal dimensions of the columns $(a)$ roughly form two c-shaped plots above and below $110 \mathrm{~cm}$ to $120 \mathrm{~cm}$. This could be due to a change from columnar to included platelet ice. The blip observed in this trend at $120 \mathrm{~cm}-130 \mathrm{~cm}$ coincides with a similar anomaly seen in the vertical resistivity data for the Antarctic. As with the Arctic models the separation between the columns $(b)$ is a mirror image of the sizing of the columns. 
The thickness of the horizontal tubes $(c)$ is relatively constant until a depth of $\sim 80 \mathrm{~cm}$ where the thickness begins to increase steadily. This is consistent with a change from columnar ice with little horizontal connectivity to included platelet ice with greater horizontal spaces available between the randomly orientated ice crystals. Between these regions ice cores indicate the presence of disrupted columnar ice (Gough, A., pers. comm., 2011), where crystals have smaller vertical extent, and there is thus an opportunity for a greater number of horizontal brine connections. In our simple model these greater number of connections could be represented as wider channels.

In the Antarctic models the dimensions of the cubes $(d)$ display a double c-shaped curve similar to that observed in the horizontal dimensions of the columns of brine.

For both models, apart from the lower part of the Antarctic data, the thicknesses of the horizontal tubes $(c)$ are significantly smaller, by approximately an order of magnitude, than the initial column size. This would seem to suggest that electrical connection may occur along grain boundaries rather than through developed brine channels. It has in fact been suggested that DC conductivity in sea ice may be controlled by the brine distribution existing at the grain boundaries (Toyama et al. 2001) and the connected liquid veins at triple junctions in the ice (Wolff et al., 1997).

There is a significant increase in the thickness of the horizontal tubes of the Arctic model on the 16-17 June to dimensions more inline with the column brine inclusions observed in the vertical direction. Additionally, as mentioned above, the thickness of the tubes increases with depth in the Antarctic model reaching 0.65 times the initial column size at depths of $170 \mathrm{~cm}$ to $180 \mathrm{~cm}$. The size of the isolated cubic brine inclusions $(d)$ is fairly large compared to the other dimensions at 5.25 to 10.9 times the initial column size. However, this large dimension is probably due to our simplistic model representing all additional brine as a single pore rather than being contained in multiple smaller pores.

From the literature values obtained for the diameter of brine inclusions in sea ice range from $0.01 \mathrm{~mm}$ to $0.38 \mathrm{~mm}$ (see section 1.2 ). These values depend on the temperature and structure of the ice. Considering this range of dimensions, and comparing to the brine model structure created for the Arctic data, the thickness of the brine columns $(a)$ could be within the range of $0.01 \mathrm{~mm}$ to $1.24 \mathrm{~mm}$. The spacing between the columns of brine (b) would then have dimensions between $0.17 \mathrm{~mm}$ and $7.41 \mathrm{~mm}$. Literature values for the 
spacing between brine layers ranges from $0.2 \mathrm{~mm}$ to $0.9 \mathrm{~mm}$, while the dimensions of ice crystals are given as a few to tens of millimetres (see section 1.2). These values fall within the upper and lower regions of the range of values suggested for the spacing between the columns of brine in the model structure. Thus the model structure could represent brine layers within crystals or inclusions between crystals, depending on the initial dimension used. Assuming literature values for the thickness of the columns of brine the dimensions of the tubes $(c)$ would be within the range of $0.38 \mu \mathrm{m}$ to $0.76 \mathrm{~mm}$. Furthermore, the dimensions of the isolated cubes of brine $(d)$ would be between $0.06 \mathrm{~mm}$ and $4.14 \mathrm{~mm}$.

Similarly, the range of values for the diameter of brine inclusions in sea ice, obtained from literature and stated above, can also give an approximate range of dimensions for the model of the brine structure of the Antarctic sea ice. The thickness of the columns of brine would lie between $0.01 \mathrm{~mm}$ and $0.53 \mathrm{~mm}$, while the spacing between them would be between $0.17 \mathrm{~mm}$ and $6.76 \mathrm{~mm}$. Furthermore, the thickness of the tubes of brine would be between $0.42 \mu \mathrm{m}$ and $0.24 \mathrm{~mm}$, and the dimensions of the isolated cubes would lie between $0.05 \mathrm{~mm}$ and $2.47 \mathrm{~mm}$. These ranges are towards the lower end of the range of dimensions given for the brine components of the Arctic models, due to there being less of a change in structure in the Antarctic sea ice over the measurement period.

Studies of the brine component of sea ice (see section 1.2) also indicate an increase in the dimensions of brine inclusions as the ice warms. Furthermore, as these brine pockets expand they can merge to form even bigger inclusions. In our simple model both these observations are consistent with the general increasing of the dimensions of the brine components, noted as the temperatures increased over the measurement period. Using the median value of the brine inclusion diameters suggested in literature (see section 1.2) it can be seen how the dimensions of the models vary over the measurement period.

In the Arctic models the horizontal dimensions of the columns would increase from $0.2 \mathrm{~mm}$ to $0.5 \mathrm{~mm}$ between early April and late May. The dimensions then increase to around $0.6 \mathrm{~mm}$ in the $16-17$ June model. The variation of the thickness of the horizontal tubes would be given by 7.6 $\mu \mathrm{m}<c<42 \mu \mathrm{m}$, between 7-9 April and 28-29 May. In mid June these dimensions jump sharply to around $0.22 \mathrm{~mm}$. Finally the sides of the cube would vary from $1.2 \mathrm{~mm}$ to $2.18 \mathrm{~mm}$. 
As mentioned above, for the Antarctic data the structure of the brine components shows no clear trend over the measurement period with the values for the models closely grouped and crossed over one another. Thus it is difficult to set a range for any increase there may be in brine inclusion size with temperature.

Despite difficulties in defining the actual sizes of the microstructure, a basic two phase structure has been constructed which models both the observed resistivities, and their variation with brine volume fraction. The proposed structure of cubic columns, tubes and cubes is an idealization of a more complex microstructure, but gives an indication of the possible form of the structure of the brine inclusions, within the studied landfast first year Arctic and Antarctic sea ice. The modelled structure suggests expanding brine inclusions with increasing temperature, vertical connections between brine inclusions even when the ice is still relatively cool, and horizontal connectivity of brine along grain boundaries. 


\subsection{Percolation theory}

The simplest form of percolation theory considers a model of a collection of points distributed in space. Whether these points are connected or not is determined by a given probability, dependent upon the parameters of the model. These models undergo a transition from a state where the connectivity of the points are localised to one in which the points become connected in an infinite cluster. This transition occurs at a critical probability. Before the transition the model is dominated by one phase, while afterwards it is dominated by the other.

As discussed previously the occurrence of percolation thresholds in sea ice have been suggested by other studies of sea ice properties. For example Golden et al. (1998), (2007) and Golden (2003) have suggested that sea ice exhibits a marked transition in its fluid transport properties when $V_{b} / V$ is about $5 \%$. For a bulk salinity of $5 \%$, Golden et al. (2007) indicate that the critical porosity (or brine volume fraction) of $5 \%$ corresponds to a temperature of $-5^{\circ} \mathrm{C}$. Thus, below $-5^{\circ} \mathrm{C}$ the ice is impermeable, however, above this temperature brine can move through the ice (Golden et al. 1998; 2007 and Golden 2003). However, as flow of electric current can occur at smaller brine inclusion diameter than fluid flow this threshold may not be directly transferable to resistivity properties. From measurements of $\rho_{H}$ on Arctic sea ice, Ingham et al. (2008) observed that their FF vs. $V_{b} / V$ plot was only consistent with Archie's Law until temperatures rose above $-5^{\circ} \mathrm{C}$. At these temperatures the brine volume fraction exceeded $8 \%-10 \%$. The authors suggest that at this stage a percolation threshold is crossed, and the bulk properties of the sea ice go from being dominated by the ice phase to being dominated by the brine phase. That estimate was based, however, on three sets of much sparser data acquired using only 2 boreholes.

As mentioned at the start of this chapter, the drop in the horizontal component of resistivity observed in the Arctic between late May and mid June appears as a sharp discontinuity in the formation factor plot (Figure 6.1a). This could suggest that a percolation threshold may have been crossed, and that above this transition (i.e. at higher brine volume fractions) the physical properties of the ice become dominated by those of the brine component. There is also a significant decrease in the formation factor plot for the vertical resistivity observed in the Arctic. This is perhaps best seen on a plot 
with a linear rather than $\log$ scale, such as the vertical $F F$ vs. $V_{b} / V$ plot for the 2008 Arctic data in Figure 6.27 below.

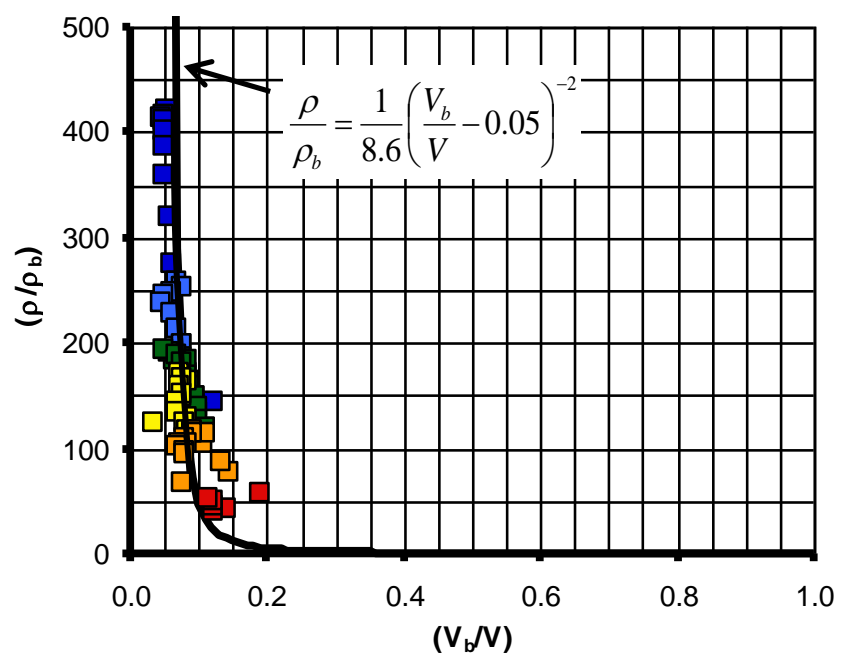

\begin{tabular}{|ll|}
\hline 7-9 Apr & $\square 25-26 \mathrm{Apr}$ \\
$\square$ 8-9 May & $\square$ 20-21 May \\
$\square$ 28-29 May & $\square$ 16-17 Jun \\
& \\
\hline Golden (pers. comm., 2009) & \\
\hline
\end{tabular}

Figure 6.27: Plot of formation factor $\left(\rho / \rho_{\mathrm{b}}\right)$ vs. brine volume fraction $\left(\mathrm{V}_{\mathrm{b}} / \mathrm{V}\right)$ for the vertical resistivity data from the 2008 Arctic measurements. Black line represents the relationship determined by Golden (pers. comm., 2009)

The shape of this plot is closely matched by a relationship determined by Golden (pers. comm., 2009) from direct measurement of the vertical resistivity of Antarctic sea ice cores. The suggested relationship between vertical FF and brine volume fraction is obtained from consideration of percolation theory and takes the form

$$
F F(\phi) \sim F F_{o}\left(\phi-\phi_{c}\right)^{-2}, \phi \rightarrow \phi_{c}^{+}
$$

where $\phi$ is the brine volume fraction, and $\phi_{c}$ the critical brine volume fraction or threshold value which is set to 0.05 , a value predicted for electrical conductivity in columnar sea ice. The scaling parameter $F F_{o}=1 / 8.6$ is obtained from the intercept of a linear best fit of the resistivity data obtained from the sea ice cores, when plotted as $\log (\phi-0.05)$ vs. $\log (F F)$.

It should be noted that most of our Arctic measurements occurred at temperatures greater than $-5^{\circ} \mathrm{C}$ (Figure 3.16) and brine volume fractions higher than $5 \%$ (Figure 3.25). This would suggest that if there is a percolation transition at the widely used threshold of a brine volume fraction of $5 \%$ most of our data are measured above the 
percolation threshold with little or no data showing the state of the ice prior to this transition. However, Ingham et al. (2008) did obtain horizontal resistivity data within this regime of lower temperature and brine volume fraction. By combining our horizontal resistivity data with that from Ingham et al. (2008) (measured in a similar locale two years earlier) a better picture of the proposed percolation transition may be obtained. A plot of the FF vs. $V_{b} / V$ of the combined data is shown in Figure 6.28 below. From this figure it can be clearly seen that there are two regions of relatively constant resistivity, at high and low brine volume fraction, connected by a region of sharply dropping resistivities between brine volume fractions of approximately $5 \%$ to $10 \%$.

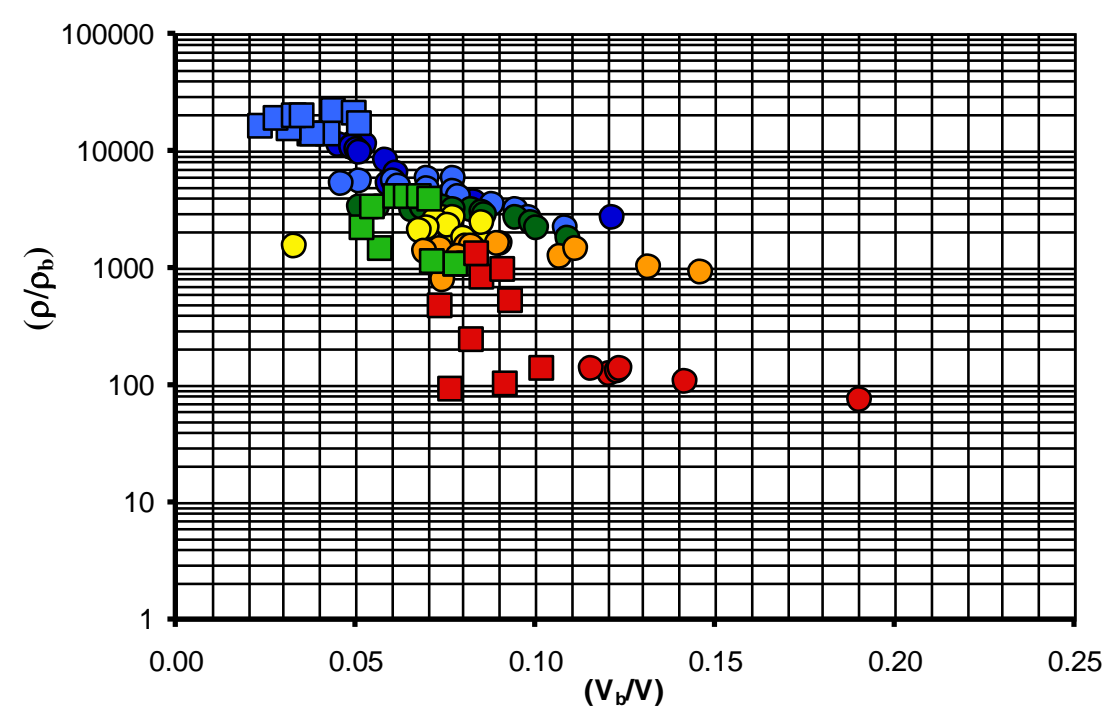

\begin{tabular}{|c|c|c|}
\hline - 7-9 Apr 2008 & O 25-26 Apr 2008 & o 8-9 May 2008 \\
\hline O 20-21 May 2008 & O 28-29 May 2008 & ○ 16-17 Jun 2008 \\
\hline 口22-25 April 2006 & $\square 11$ May 2006 & $\square 8$ Jun 2006 \\
\hline
\end{tabular}

Figure 6.28: Plot of formation factor $\left(\rho / \rho_{b}\right)$ vs. brine volume fraction $\left(V_{b} / V\right)$ for the horizontal resistivity data from the 2008 Arctic measurements (circles), combined with data from Ingham et al. (2008) (squares).

One way of modelling this change of phase or percolation transition is to consider a 3D network of resistors to represent the brine structure of the sea ice. Other authors, e.g. Kirkpatrick (1973) and Bahr (1997), have considered resistor networks as models for transport properties in two phase systems, especially those exhibiting a percolation threshold. Recently, as part of a Honours degree, a research project a Victoria University of Wellington investigated the use of resistor networks to model resistivity measurements made on sea ice (McCann, D., pers. comm., 2009), and our 3D network 
builds on this research. Initially each resistor in the network is assigned a relatively high resistance to signify the small amount of brine contained along ice grain boundaries. Resistors are chosen randomly and the resistance reduced to symbolize the creation of a brine inclusion. As more and more resistors are decreased a sudden decrease in the resistance of the network is observed, see Figure 6.29. This nicely models a percolation transition, with a change from high to low resistance representing a system whose bulk properties are dominated by one phase reaching a critical threshold point, after which the bulk properties are dominated by the other phase.

\section{Resistance of a 3D resistor network as resistors are randomly changed from high to low resistance}

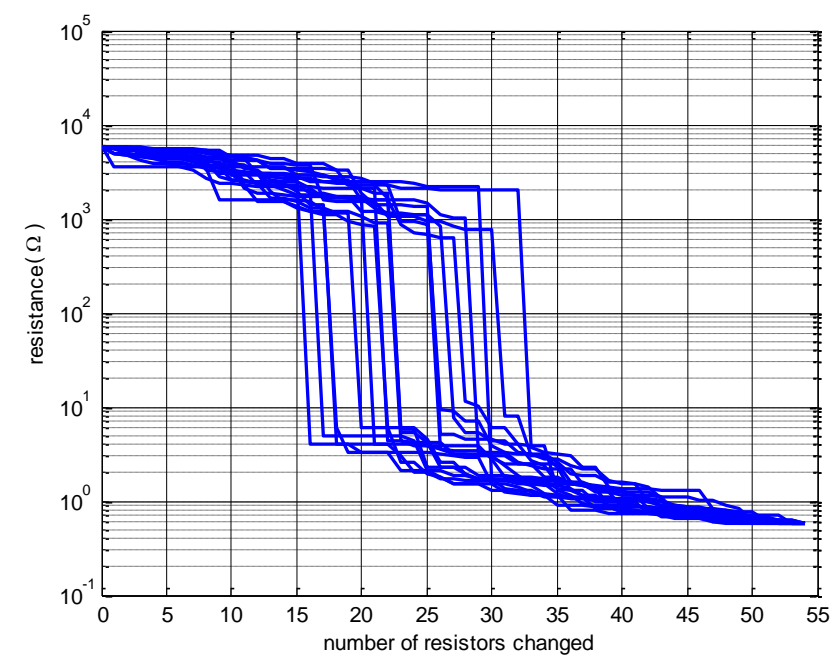

Figure 6.29: Plot of resistance vs. number of resistors changed, showing a percolation transition as more and more resistors are changed from high to low resistance.

We consider a 2 by 2 by 2 cube of resistors (Figure 6.30) where each resistor is itself a sub-array of a 2 by 2 by 2 cube of resistors, giving a total of 2916 resistors or possible brine inclusions.

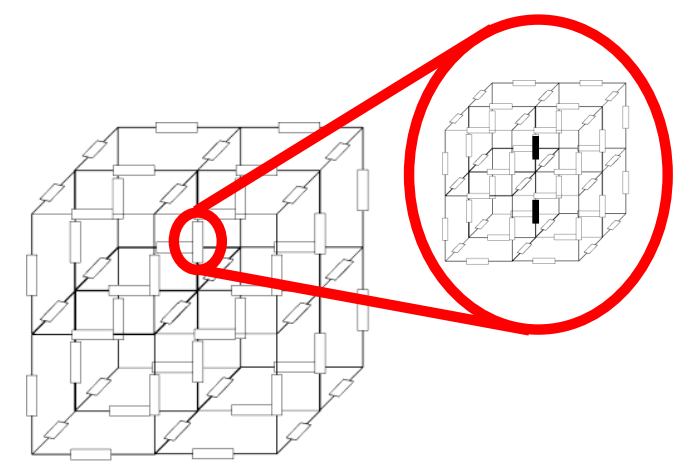

Figure 6.30: A $2 \times 2 \times 2$ resistor network where each resistor is itself represented by a $2 \times 2 \times 2$ sub-array of resistors. Black shaded resistors are present in the two centre resistors, of the outer array, and are set to a medium resistance from start. 
By using network equations to calculate both the vertical and horizontal resistance of the $3 \mathrm{D}$ resistor network, while changing the resistors from high to low resistance, it is possible to compare the response of the resistor network to that of the measured vertical and horizontal resistivity of the Arctic sea ice. However, in order to do this the results from the resistor networks need to be changed from 'resistances' and 'number of resistors changed' to 'formation factor' and 'brine volume fraction'. The simplest way of doing this is to consider the size of the brine inclusions and the volume that the $3 \mathrm{D}$ network represents. The size of the brine inclusions can be provided using values obtained from literature (see section 1.2), slightly modified to match the observed resistivities. The volume represented by the resistor network can be obtained by considering brine inclusion densities supplied by literature (see section 1.2).

Since sea ice will contain some brine inclusions (rather than simply brine contained in spaces along grain boundaries) right from formation, and these will generally be preferentially elongated vertically, the considered 3D resistor network does not initially have all resistors set to high resistance. Instead, in each of the two centre resistors of the outer array the two centre resistors of the sub arrays (black shaded resistors in Figure 6.30) are set to an intermediate value of resistance to represent this early brine inclusion element of the sea ice. The introduction of a number of vertical resistors assigned lower values means that the initial structure is anisotropic, with lower values measured in the vertical direction than in the horizontal.

The resistor network is considered to represent a volume of $1 \mathrm{~cm}^{3}$ (giving a brine inclusion density of $\sim 3$ per $\mathrm{mm}^{3}$ ), and a resistivity of $0.2658 \Omega \mathrm{m}$ is used for the brine component (average value of the brine resistivities obtained during measurements). It is found that by increasing from a brine component concentrated along grain boundaries, diameter $\sim 9.3 \mu \mathrm{m}$ and length $\sim 0.8 \mathrm{~mm}$, and some small brine inclusions of size, diameter $\sim 0.25 \mathrm{~mm}$ and length $\sim 0.8 \mathrm{~mm}$, to brine inclusions of size diameter $\sim 0.29 \mathrm{~mm}$ and length $\sim 0.8 \mathrm{~mm}$, the following plots of the response of the 3D resistor network are obtained. Figure 6.31 shows the results of the calculated changes in horizontal and vertical formation factor, of the resistor network, for 50 repeats of allowing all the resistors to randomly change from small to large brine inclusions (i.e. high to low resistance). 
(a) Horizontal resistivity

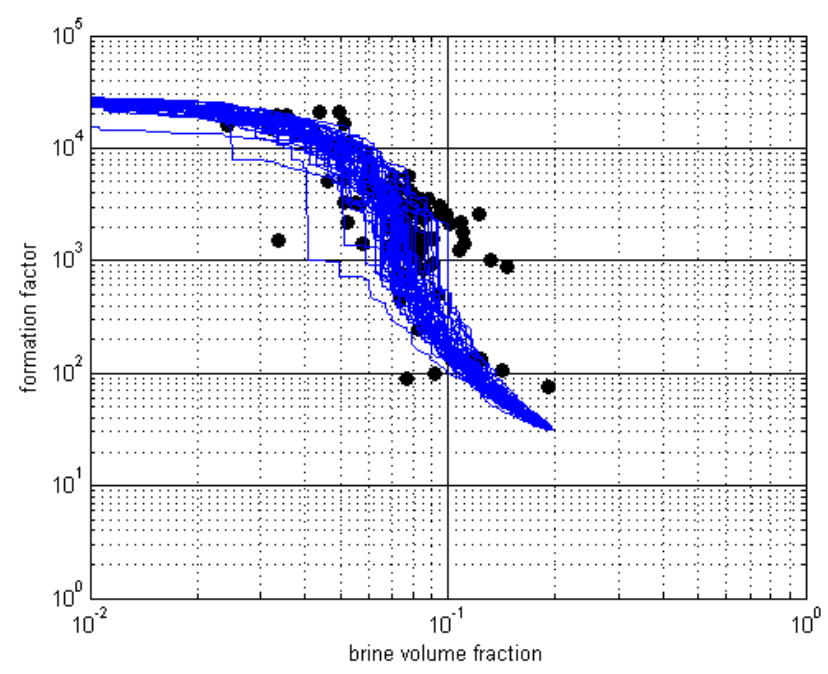

(b) Vertical resistivity

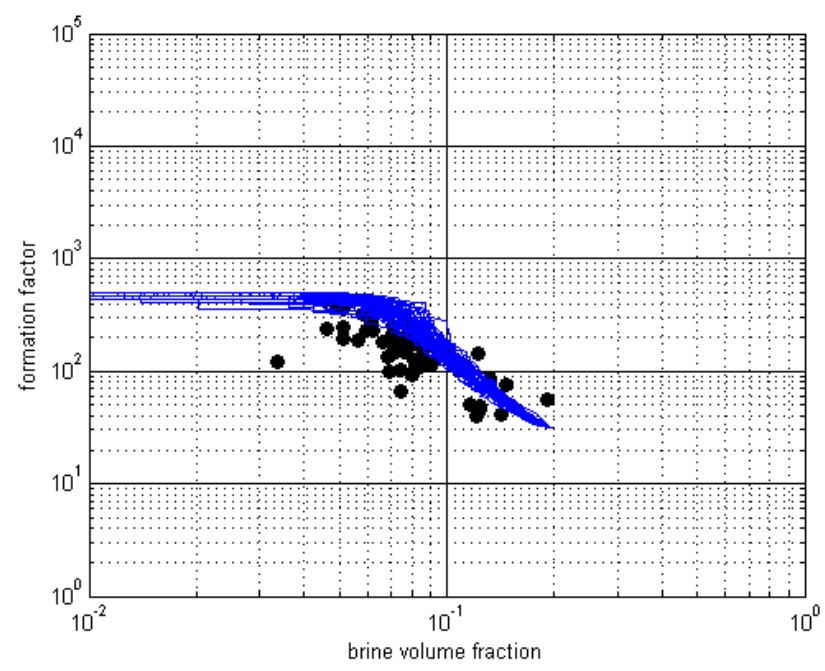

Figure 6.31: Plot of formation factor $\left(\rho / \rho_{b}\right)$ vs. brine volume fraction $\left(V_{b} / V\right)$ for resistivity of the 3D resistor network (50 repeats) and data from measurements on Arctic sea ice. (a) the horizontal resistvity, (b) the vertical resistivity.

From Figure 6.31 it can be seen that the behaviour of a 3D resistor network, as resistors are changed from high to low resistance, can closely describe the trend seen in both the horizontal and vertical resistivity structure of Arctic sea ice. Providing, the resistor network has an anisotropic resistivity structure and sensible brine inclusion sizes (determined by the resistivity of brine and the resistance assigned to the resistors). This match between the resistor network data and the measured resistivities suggests that the observed drop in the horizontal formation factor is indeed consistent with a percolation transition which occurs at a brine volume fraction of around $5 \%-10 \%$. 


\section{Chapter 7 Summary}

This thesis investigates the use of cross-borehole direct current (DC) electrical resistivity techniques as a non-destructive method of studying the anisotropic brine microstructure of first year sea ice and its evolution with changes in temperature and salinity.

Previous resistivity studies of sea ice (e.g. Thyssen et al., 1974; Timco, 1979; Buckley et al., 1986; Ingham et al., 2008) have shown that surface soundings do not differentiate between the vertical and horizontal components of the anisotropic bulk resistivity structure. Furthermore, the thickness of the anisotropic ice cover is underestimated making the interpretation of surface soundings, in terms of the variation of the measured geometric mean resistivity with depth, problematic.

Ingham et al. (2008) have demonstrated that a DC resistivity technique, which makes use of cross-borehole tomography, can be used to measure the horizontal component of the anisotropic structure of first year sea ice. This thesis expands on this technique to allow investigation of both the horizontal and vertical resistivity structure of the sea ice.

It is shown theoretically (Chapter 2) that the horizontal resistivity structure of the undisturbed ice between boreholes can be accurately recovered by using two boreholes each containing one potential and one current electrode. These electrodes must be positioned such that the vertical spacing between electrodes is significantly smaller than the horizontal spacing of the boreholes. Furthermore, theory shows (Chapter 2) that measurements aimed at determining the geometric mean resistivity require three or four electrodes each positioned at approximately the same depth in separate boreholes. Although it is shown that exact measurement of the geometric mean resistivity is not possible, reasonable estimates of the true resistivity can be retrieved from numerical modelling. These values of the horizontal and geometric mean resistivity structure then allow estimates of the vertical resistivity of sea ice, which cannot be directly measured, to be calculated.

The measurement technique is illustrated by the results of a series of measurements made in first year sea ice at sites in the Arctic and the Antarctic (see sections 3.1 and 
3.2). Six measurement sets were made at one - two weekly intervals over April - June off the coast of Barrow, Alaska in 2008. While a further four measurement sets were obtained over two weeks in November 2009 in McMurdo Sound off Ross Island, Antarctica. The obtained resistivity structure of the Arctic sea ice (see section 5.1.1) clearly shows variation over the measurement period as the temperatures increase. The observed general decreasing of resistivities indicate an increase of the connectivity of the brine inclusions especially in the horizontal direction. The results show a possible percolation transition, with a significant decrease in resistivity indicating a change from a medium dominated by the ice phase to one dominated by the brine phase. The measurements on the Antarctic sea ice (see section 5.2) return a resistivity structure that suggests a change in structure with depth. This change does not correspond with any significant changes in temperature, salinity or brine volume fraction and has been attributed to a change in ice type from columnar ice to included platelet ice.

It should be noted that there are large uncertainties in the geometric mean resistivities retrieved from the numerical modelling. Furthermore these values are obtained via consideration of synthetic datasets created for an idealised representation of sea ice consisting of two boundaries between which there is a uniform material, whereas the structure of real sea ice may include a variation of the coefficient of anisotropy and resistivity with depth. However, when considering the difference in resistivity of a medium of isolated brine inclusions and one with a connected brine network several orders of magnitude are involved. Thus resolution of this important change in structure can be obtained from the resistivity data, despite the approximations used.

The analysis of cores of Antarctic sea ice (Gough, A., pers. comm., 2011) gives depths for when platelet ice begins to appear in the ice cover. These depths are similar to those at which changes in resistivity structure, associated with a change from columnar to included platelet ice, are observed. Suggesting that the DC electrical resistivity technique used can differentiate between ice types.

Basic cubic two phase models, whose resistivity response and brine volume fraction values match the measured values of the sea ice, have been produced (Chapter 6). While such models are a highly idealized representation of the complex brine microstructure they provide insight into the typical characteristics of the brine inclusions. The models consisting of vertical columns, horizontal tubes and isolated cubes of brine show the expected increasing brine network as temperatures increase. The dimensions of the 
horizontal tubes are significantly smaller than those of the vertical columns, suggesting that while the measured vertical resistivities requires connection between vertically aligned brine inclusions the horizontal resistivity values are due to connections along grain boundaries.

In future it may be beneficial to obtain more frequent measurements, in particular to better define the significant drop in resistivity that was observed in the Arctic measurement set between late May and mid June. Additionally most of our Arctic data was recorded when the temperature of the ice was above $-5^{\circ} \mathrm{C}$ and the brine volume fractions were greater than $5 \%$. Thus for consideration of the percolation threshold the horizontal resistivity data was combined with colder and lower brine volume fraction measurements made by Ingham et al. (2008). Ideally in further fieldwork measurements would be obtained earlier in the season in colder ice to provide data on sea ice when its properties are dominated by the ice phase. Measurements earlier in the season may also allow observation of ice growth. Further investigation of why the region of initial frazil ice growth, observed at the surface of ice cores (Eicken, H., pers. comm., 2010), does not seem to be resolved in resistivity profiles of the Arctic ice may also be warranted.

Unlike the Arctic data, the short measurement period available for the Antarctic data did not allow observation of changes in the sea ice microstructure over time. Thus in future work it would be interesting to carry out a longer set of measurements so this evolution can also be studied in the Antarctic region. From our Antarctic results it seems that our measurement technique can differentiate between different ice types. However, this requires a more detailed study with greater comparisons of ice core and resistivity data and perhaps longer electrode strings, to better cover the region containing a majority of platelet ice.

In general obtaining more data over future studies would help define relationships between the measured resistivity and the temperature, salinity and brine volume fraction of the ice. This would allow these properties to be determined from our non-destructive measurement technique. To increase the ease of obtaining the resistivity measurements, future work could look at creating an automated measuring system. This would involve additional 'smart' electrodes, the design of a new datalogger system, and consideration of battery and data storage and retrieval needs. Additional work on resolving the horizontal anisotropy of sea ice and the implications of this structure should also be considered in the future. 
As mentioned above, our brine models provide a valuable insight into the microstructure of the sea ice, however, they are only a simplistic picture of the brine structure. For example these models do not differentiate between one wide tube and two thinner tubes of $1 / 2$ the cross sectional area. Thus the appearance of more brine inclusions may be observed in our models as a thickening of the brine components. The design of more complicated or sophisticated models requires further consideration.

This current research has provided results which could enhance the study of sea ice. For instance being able to better estimate and track the resistivity of sea ice could aide in the interpretation of EM surveys, used for remote sensing and ice thickness measurements. Knowing the approximate structure of the brine may allow better determination of the optical properties of the ice, as this property is affected by scattering from brine inclusions. Additionally it may be possible to estimate the strength of the ice, as this is related to brine volume values. Our models of the brine structure may also provide a means of estimating the thermal conductivity of the sea ice.

In conclusion this research has developed an in situ DC electrical resistivity crossborehole tomography technique which contributes significantly to the understanding of sea ice microstructure and its response to changes in temperature and salinity. Furthermore, the measurement method holds promise as a way of differentiating between different ice types. As the structure of sea ice affects the physical properties of the ice it is important to be able to study the sea ice structure to gain a fuller understanding of the role sea ice plays in a range of contexts. 


\section{Appendix I \\ Resistivity on a medium with horizontal anisotropy}

From section 2.1.2 equation (2.5) gives the potential in an anisotropic medium as

$$
V \frac{C}{\left(x^{2} \rho_{x}+y^{2} \rho_{y}+z^{2} \rho_{z}\right)^{1 / 2}}
$$

where $C$ is a constant, $x, y$ and $z$ are the distances between where the current is injected and the potential is measured, along the respective axes, and $\rho_{i}$ is the resistivity in the $i$ direction

Thus using equation (I a) the current densities given in equations (2.4) in section 2.1.2 become

$$
\begin{aligned}
& J_{x}=\frac{x C}{\left(x^{2} \rho_{x}+y^{2} \rho_{y}+z^{2} \rho_{z}\right)^{3 / 2}} \\
& J_{y}=\frac{y C}{\left(x^{2} \rho_{x}+y^{2} \rho_{y}+z^{2} \rho_{z}\right)^{3 / 2}} \\
& J_{z}=\frac{z C}{\left(x^{2} \rho_{x}+y^{2} \rho_{y}+z^{2} \rho_{z}\right)^{3 / 2}}
\end{aligned}
$$

with the total current density given by

$$
J=\left(J_{x}{ }^{2}+J_{y}{ }^{2}+J_{z}{ }^{2}\right)^{1 / 2}=\frac{\left(x^{2}+y^{2}+z^{2}\right)^{1 / 2} C}{\left(x^{2} \rho_{x}+y^{2} \rho_{y}+z^{2} \rho_{z}\right)^{3 / 2}}
$$

Changing equation ( $\mathrm{I} \mathrm{b}$ ) to be in spherical coordinates gives

$$
J=\frac{C}{r^{2}\left[\sin ^{2} \theta\left(\rho_{x}+\sin ^{2} \phi\left\{\rho_{y}-\rho_{x}\right\}-\rho_{z}\right)+\rho_{z}\right]^{3 / 2}}
$$

Where $C$ is a constant, $\rho_{i}$ is the resistivity in the $i$ direction, $r$ is the distance between where the current is injected and the potential measured, $\theta$ is the angle from the vertical to the vector $r$, and $\phi$ is the horizontal angle from the $\mathrm{x}$ coordinate axis to the horizontal projection of the vector $r$.

For an electrode buried in an anisotropic medium, such as sea ice, the current must pass through a sphere of radius $r$ centred on the electrode. In this case Bhattacharya and Patra (1968) show that the current at the electrode is given by 


$$
I=\int_{s} J \cdot d s=\int_{0}^{2 \pi} \int_{0}^{\pi} J r^{2} \sin \theta d \theta d \phi
$$

Inserting equation (I c) into the expression above and solving and simplifying gives $I=\frac{4 \pi C}{\sqrt{\rho_{x} \rho_{y} \rho_{z}}}$ which rearranges to $C=\frac{I \sqrt{\rho_{x} \rho_{y} \rho_{z}}}{4 \pi}$

Substituting $\mathrm{C}$ into equation (I a) gives

$$
V=\frac{I \sqrt{\rho_{x} \rho_{y} \rho_{z}}}{4 \pi \sqrt{x^{2} \rho_{x}+y^{2} \rho_{y}+z^{2} \rho_{z}}}=\frac{I \sqrt{\rho_{x} \rho_{y}}}{4 \pi \sqrt{x^{2}\left(\frac{1}{\lambda_{x}}\right)^{2}+y^{2}\left(\frac{1}{\lambda_{y}}\right)^{2}+z^{2}}} \text { (I d) }
$$

where $x, y$ and $z$ are the distances, between where the current is injected and the potential measured, as measured along the relative axis, $\rho_{i}$ is the resistivity in the $i$ direction, and $\lambda_{x}$ and $\lambda_{y}$ are given by $\lambda_{x}=\sqrt{\rho_{z} / \rho_{x}}, \lambda_{y}=\sqrt{\rho_{z} / \rho_{y}}$.

If measurements had been made on an isotopic medium then the potential would be given by $V=\frac{I \rho}{4 \pi \sqrt{x^{2}+y^{2}+z^{2}}}$. The conditions under which expression (I d) reduces to the isotropic expression, when $\rho$ is considered to be $\rho_{x}, \rho_{y}$ or $\rho_{z}$ indicates whether the relevant resistivity can be determined. For example, in order to measure $\rho_{x}$ requires that

$$
z=\sqrt{\frac{x^{2}\left(1-\frac{\rho_{x}}{\rho_{y} \lambda_{x}{ }^{2}}\right)+y^{2}\left(1-\frac{\rho_{x}}{\rho_{y} \lambda_{y}{ }^{2}}\right)}{\frac{\rho_{x}}{\rho_{y}}-1}}
$$

Similar expressions are found for $\rho_{y}$ or $\rho_{z}$. As these expressions can not be solved with a real value of $\lambda_{i}$, it seems that the individual components of the bulk resistivity of a medium with vertical and horizontal anisotropy can not be measured.

For instance, using expression ( $\mathrm{I} d$ ) consider the potential measured in two perpendicular directions eg along the $\mathrm{x}$-axis and the $\mathrm{y}$-axis. In these two situations the expression for the potential would become $V_{x}=\frac{I \sqrt{\rho_{x} \rho_{y}}}{4 \pi \sqrt{x^{2}\left(\frac{1}{\lambda_{x}}\right)^{2}+z^{2}}}$ and 
$V_{y}=\frac{I \sqrt{\rho_{x} \rho_{y}}}{4 \pi \sqrt{y^{2}\left(\frac{1}{\lambda_{y}}\right)^{2}+z^{2}}}$. Thus, for cross-borehole measurements in a medium with anisotropic horizontal resistivity (i.e. $\rho_{x} \neq \rho_{y}$ ) the potential in two perpendicular directions are each dependent on both $\rho_{x}$ and $\rho_{y}$. Thus cross-borehole measurements do not give information on the horizontal anisotropy of a medium

However, consider current from an electrode placed at the surface of an anisotropic medium, such as sea ice. In this case the current must pass through a hemisphere of radius $r$ centred on the electrode, and the total current flowing out through this surface (and thus the current at the electrode) is given by

$$
I=\int_{s} J \cdot d s=\int_{0}^{2 \pi} \int_{\pi / 2}^{\pi} J r^{2} \sin \theta d \theta d \phi
$$

Inserting equation (I c) into the expression above and solving and simplifying gives $I=\frac{2 \pi C}{\sqrt{\rho_{x} \rho_{y} \rho_{z}}}$ which rearranges to $C=\frac{I \sqrt{\rho_{x} \rho_{y} \rho_{z}}}{2 \pi}$

Substituting $\mathrm{C}$ into equation ( $\mathrm{I}$ a) gives

$$
V=\frac{I \sqrt{\rho_{x} \rho_{y} \rho_{z}}}{2 \pi \sqrt{x^{2} \rho_{x}+y^{2} \rho_{y}}}
$$

where $x, y$ and $z$ are the distances, between where the current is injected and the potential measured, as measured along the relative axis ( $z$ being zero as the electrodes are at the surface), $\rho_{i}$ is the resistivity in the $i$ direction, and $\lambda_{x}$ and $\lambda_{y}$ are given by $\lambda_{x}=\sqrt{\rho_{z} / \rho_{x}}, \lambda_{y}=\sqrt{\rho_{z} / \rho_{y}}$.

Once again consider the potential measured in two perpendicular directions e.g. along the $\mathrm{x}$-axis and the $\mathrm{y}$-axis. In these two situations the expression for the potential would become $V_{x}=\frac{I \sqrt{\rho_{y} \rho_{z}}}{2 \pi x}$ and. $V_{y}=\frac{I \sqrt{\rho_{x} \rho_{z}}}{2 \pi y}$ Thus, for surface measurements on a medium with anisotropic horizontal resistivity (i.e. $\rho_{x} \neq \rho_{y}$ ) the potential in two perpendicular directions are each dependent on different components of the horizontal resistivity. Measurements along the $\mathrm{x}$-axis will provided information on $\rho_{y}$ and those along the y-axis will provide information on $\rho_{x}$. Thus surface resistivity soundings can 
be used to determine if there is any horizontal anisotropy in the medium on which measurements are being made, by comparing two perpendicular sets of measurements. Surface soundings running approximately parallel and perpendicular to the coast at Barrow, Alaska indicate little horizontal anisotropy in the sea ice. 


\title{
Appendix II \\ Example of format of files used to read the data into the 3D inversion program RES3DINV
}

\author{
(see section 4.2.1)
}

Barrow sea-ice
$07-09 / 04 / 08$
horizontal resistivity
inversion
11
11
0.1
0.1
13

Number of boreholes 4

Borehole 1

18

0.00 .00 .1

0.00 .00 .2

0.00 .00 .3

0.00 .00 .4

0.00 .00 .5

0.00 .00 .6

0.00 .00 .7

0.00 .00 .8

0.00 .00 .9

0.00 .01 .0

0.00 .01 .1

0.00 .01 .2

0.00 .01 .3

0.00 .01 .4

0.00 .01 .5

0.00 .01 .6

0.00 .01 .7

0.00 .01 .8

Borehole 2

18

1.00 .00 .1

1.00 .00 .2

1.00 .00 .3

1.00 .00 .4

1.00 .00 .5

1.00 .00 .6

1.00 .00 .7

1.00 .00 .8

1.00 .00 .9

1.00 .01 .0

1.00 .01 .1
I

I

I

ITitle

I surface grid size in $\mathrm{x}$ direction

I surface grid size in y direction

Ix unit electrode spacing

¡y unit electrode spacing

I array number - 13 for borehole survey with resistance data

IHeader

'number of boreholes

Header for $1^{\text {st }}$ borehole

, number of electrodes in borehole 1

$\mathrm{Ix}, \mathrm{y}$ and $\mathrm{z}$ location of $1^{\text {st }}$ electrode in borehole

$\mathbf{I}_{\mathrm{X}}, \mathrm{y}$ and $\mathrm{z}$ location of $2^{\text {nd }}$ electrode in borehole

$\mathrm{I}, \mathrm{y}$ and $\mathrm{z}$ location of $3^{\text {rd }}$ electrode in borehole

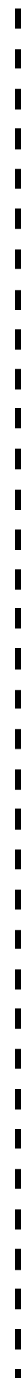


1.00 .01 .2

1.00 .01 .3

1.00 .01 .4

1.00 .01 .5

1.00 .01 .6

1.00 .01 .7

1.00 .01 .8

Borehole 3

18

1.01 .00 .1

1.01 .00 .2

1.01 .00 .3

1.01 .00 .4

1.01 .00 .5

1.01 .00 .6

1.01 .00 .7

1.01 .00 .8

1.01 .00 .9

1.01 .01 .0

1.01 .01 .1

1.01 .01 .2

1.01 .01 .3

1.01 .01 .4

1.01 .01 .5

1.01 .01 .6

1.01 .01 .7

1.01 .01 .8

Borehole 4

18

0.01 .00 .1

0.01 .00 .2

0.01 .00 .3

0.01 .00 .4

0.01 .00 .5

0.01 .00 .6

0.01 .00 .7

0.01 .00 .8

0.01 .00 .9

0.01 .01 .0

0.01 .01 .1

0.01 .01 .2

0.01 .01 .3

0.01 .01 .4

0.01 .01 .5

0.01 .01 .6

0.01 .01 .7

0.01 .01 .8

Header for $3^{\text {rd }}$ borehole

Inumber of electrodes in borehole 3

the following section gives the $\mathrm{x}, \mathrm{y}$ and $\mathrm{z}$ location of the electrodes in the borehole

IHeader for $4^{\text {th }}$ borehole

Inumber of electrodes in borehole 4

the following section gives the $\mathrm{x}, \mathrm{y}$ and $\mathrm{z}$ location of the electrodes in the borehole

2418

I number of data points

$\begin{array}{lllllllllllll}4 & 0 & 0 & 0.1 & 0 & 1 & 0.1 & 0 & 0 & 0.2 & 0 & 0.2 & 160.38\end{array}$, for each datum point, the first

$\begin{array}{lllllllllllll}4 & 0 & 0 & 0.1 & 0 & 1 & 0.1 & 0 & 0 & 0.3 & 0 & 0.3 & 159.62 \\ \text {,parameter is the number of }\end{array}$ 


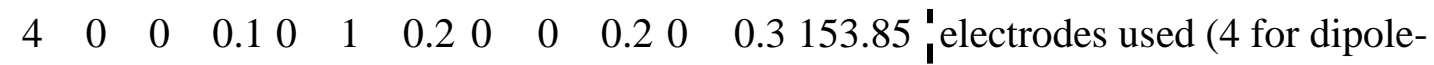

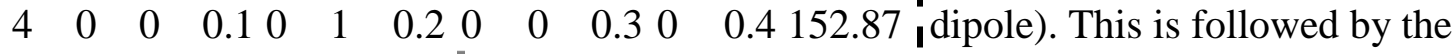
$\mathrm{I} x, \mathrm{y}$ and $\mathrm{z}$ location of the current Ielectrode $\mathrm{C} 1$, the current Ielectrode $\mathrm{C} 2$, the potential Electrode P1 and the potential $\begin{array}{lllllllllllllll}4 & 1 & 1 & 1.8 & 0 & 1 & 1.8 & 1 & 1 & 1.6 & 0 & 1.6 & 0.279 & \text { jelectrode P2. The last value is }\end{array}$ $\begin{array}{llllllllllllll}4 & 1 & 1 & 1.8 & 0 & 1 & 1.8 & 1 & 1 & 1.7 & 0 & 1.7 & 0.545 & \text { I the measured resistance }\end{array}$

$\begin{array}{ll}\text { FIXED REGIONS } & \text { 'Header to indicate model has fixed regions } \\ 1 & \text { Inumber of regions to be fixed } \\ \mathrm{R} & \text { Ix, y, z coordinates of top-left-back corner } \\ 0.0,0.0,1.3 & \mathbf{I}, \mathrm{y}, \mathrm{z} \text { coordinates of bottom-right-front corner } \\ 1.0,1.0,1.8 & \text { 'resistivity of fixed region } \\ 0.4 & \text { Iamping factor weight for fixed region } \\ 2 & \text { the end of the data file is indicated by a few zero's } \\ 0 & \text { I } \\ 0 & \end{array}$





\section{Appendix III \\ Publications and conference presentations relating to this research}

Below is a summary of the publications and conference presentations that this research has produced, at time of thesis submission.

\section{Publications}

Jones, K., Ingham, M., Pringle, D., Eicken, H., 2011. Cross-borehole resistivity tomography of Arctic and Antarctic sea ice, Annals. Glac., 52, 161-168.

Jones, K. A., Ingham, M., Pringle, D. J., Eicken, H., 2010. Temporal variations in sea ice resistivity: Resolving anisotropic microstructure through cross-borehole DC resistivity tomography, J. Geophys. Res., 115, C11023. (doi:10.1029/2009JC006049)

\section{Conference presentations}

Ingham, M., Jones, K., 2011. The temporal evolution of sea ice microstructure, IUGG Earth on the edge: science for a sustainable planet, Melbourne, Australia, 28 June -7 July, 2011.

Jones, K., Ingham, M., Pringle, D., Eicken, H., 2010. Resolving sea-ice microstructure using cross-borehole resistivity tomograph, International Symposium on Sea Ice in the Physical and Biogeochemical System, Troms $\varnothing$, Norway, 31 May - 4 June, 2010.

Jones, K., Ingham, M., Pringle, D., Eicken, H., 2010. Using In Situ Cross-Borehole Resistivity Tomography to Resolve the Microstructure of Arctic and Antarctic Sea Ice, International Polar Year - Oslo Science Conference, Oslo, Norway, 8-12 June, 2010.

Jones, K., Ingham, M., Pringle, D., Eicken, H., 2010. Cross borehole electrical resistivity tomography of Arctic and Antarctic sea ice, New Zealand Sea Ice Symposium, University of Otago, Dunedin, New Zealand, 18-19 February 2010.

Ingham, M., Jones, K., Pringle, D. J., Eicken, H., 2009. Resolution of sea ice microstructure using cross borehole resistivity tomography. Fall Meeting, American Geophysical Union, San Francisco, 14-18 December 2009.

Jones, K., Ingham, M., Pringle, D., Eicken, H., 2009. Resolving sea ice microstructure using cross borehole resistivity tomography, NZIP 2009 Conference, University of Canterbury, Christchurch, New Zealand, 6-8 July 2009. 



\section{References}

Addison, J. R., 1969. Electrical properties of saline ice, J. Appl. Phys., 40, 3105-3114.

Archie, G. E., 1942. The electrical resistivity $\log$ as an aid in determining some reservoir characteristics, Trans. Am. Inst. Min. Metall. Pet. Eng., 146, 54-62.

Bahr, K., 1997. Electrical anisotropy and conductivity distribution functions of fractal random networks and of the crust: the scale effect of connectivity, Geophys. J. Int., 130, 649-660.

Bhattacharya, P. K., Patra, H. P., 1968. Direct current geoelectric sounding: Principles and interpretation, Elsevier Publishing, Amsterdam.

Birchak, J. R., Gardner, L. G., Hipp, J. W., Victor, J. M., 1974. High dielectric constant microwave probes for sensing soil moisture, Proceedings of the IEEE, 62(1), 93-98.

Buckley, R. G., Staines, M. P., Robinson, W. H., 1986. In situ measurements of the resistivity of Antarctic sea ice, Cold Reg. Sci. Technol., 12, 285-290.

Callaghan, P. T., Dykstra, R., Eccles, C. D., Haskell, T. G., Seymour, J. D., 1999. A nuclear magnetic resonance study of Antarctic sea ice brine diffusivity, Cold Reg. Sci. Technol., 29, 153-171.

Chelidze, T. L., Gueguen, Y., 1999. Electrical spectroscopy of porous rocks: a review I. Theoretical models, Geophys. J. Int., 137, 1-15.

Cherkaeva, C., Golden, K., 1998. Inverse bounds for microstructural parameters of composite media derived from complex permittivity measurements, Waves in Random and Complex Media, 8, 437-450.

Comiso, J. C., 2003. Large scale characteristics and variability of the global sea ice cover, In: Thomas, D. N., Dieckmann, G. S. (Eds), Sea Ice: An introduction to its Physics, Chemistry, Biology and Geology, Blackwell Science Ltd, Oxford, pp112-142.

Cox, G. F. N., and Weeks, W. F., 1983. Equations for determining the gas and brine volumes in sea-ice samples, J. Glaciol., 29, 306-316.

deGroot-Hedlin, C., Constable, S., 1990. Occam's inversion to generate smooth, twodimensional models from magnetotelluric data, Geophysics, 55, 1613-1624.

Dempsey, D. E., Langhorne, P. J., Robinson, N. J., Williams, M. J. M., Haskell, T. G., Frew, R. D., 2010. Observation and modelling of platelet ice fabric in McMurdo Sound, Antarctica, J. Geophys. Res., 115, C01007.

Doi: $10.1029 / 2008 J C 005264$.

Dieckmann, G. S., Hellmer, H. H., 2003. The importance of sea ice: An overview, In: Thomas, D. N., Dieckmann, G. S. (Eds), Sea Ice: An introduction to its Physics, Chemistry, Biology and Geology, Blackwell Science Ltd, Oxford, pp1-21. 
Druckenmiller, M. L., Eicken, H., Johnson, M. A., Pringle, D. J., Williams, C. C., 2009. Towards an integrated coastal sea-ice observatory: System components and a case study at Barrow, Alaska, Cold Reg. Sci. Technol., 56, 61-72.

Eicken, H., 1992. Salinity Profiles of Antarctic Sea Ice: Field Data and Model Results, J. Geophys. Res., 97(C10), 15545-15557.

Eicken, H., 2003. From the microscopic, to the macroscopic, to the regional scale: Growth, microstructure and properties of sea ice, In: Thomas, D. N., Dieckmann, G. S. (Eds), Sea Ice: An introduction to its Physics, Chemistry, Biology and Geology, Blackwell Science Ltd, Oxford, pp22-81.

Eicken, H., Bock, C., Wittig, R., Miller, H., Poertner, H. -O., 2000. Magnetic resonance imaging of sea ice pore fluids: methods and thermal evolution of pore microstructure, Cold Reg. Sci. Technol., 31, 207-225.

Freitag, J., Eicken, H., 2003. Melt water circulation and permeability of Arctic summer sea ice derived from hydrological field experiments, J. Glaciol., 49, 349-358.

Doi: 10.3189/172756503781830601

Fricke, H., 1924. A mathematical treatment of the electric conductivity and capacity of disperse systems I: The electric conductivity of a suspension of homogeneous spheroids, Phys. Rev., 4, 575-587.

Fritsen, C. H., Lytle, V. I., Ackley, S. F., Sullivan, C. W., 1994. Autumn bloom of Antarctic pack-ice algae, Science, 266, 782-784.

Golden, K.M., 2003. Critical behavior of transport in sea ice, Phys. B Condens. Matter, 338, 274-283.

Doi: $10.1126 /$ science.282.5397.2238

Golden, K. M., Ackley, S. F., Lytle, V. I., 1998. The percolation phase transition in sea ice, Science, 282, 2238-2241.

Golden, K. M., Eicken, H., Heaton, A. L., Miner, J., Pringle, D., J., Zhu, J., 2007. Thermal evolution of permeability and microstructure in sea ice, Geophysical Research Letters, 34, L16501.

Doi:10.1029/2007GL030447

Gränicher, H., Jaccard, C., Scherrer, P., Steinemann, A., 1957. Dielectric relaxation and the electrical conductivity of ice crystals, Discuss. Faraday Soc., 23, 50 - 62.

Doi: 10.1039/DF9572300050

Grenfell, T. C., Perovich, D. K., 2004. Seasonal and spatial evolution of albedo in a snow-ice-land-ocean environment, J. Geophys. Res., 109, C01001.

Doi:10.1029/2003JC001866

Grimm, R. E., Stillman, D. E., Dec, S. F., Bullock, M. A., 2008. Low-Frequency Electrical Properties of Polycrystalline Saline Ice and Salt Hydrates, J. Phys. Chem. B, 112, 15382-15390. 
Haas, C., 2003. Dynamics versus thermodynamics: The sea ice thickness distribution, In: Thomas, D. N., Dieckmann, G. S. (Eds), Sea Ice: An introduction to its Physics, Chemistry, Biology and Geology, Blackwell Science Ltd, Oxford, pp82-111.

Haas, C., Gerlandz, S., Eicken, H., Miller, H., 1997. Comparison of sea-ice thickness measurements under summer and winter conditions in the Arctic using a small electromagnetic induction device, Geopyhsics, 62, 749-757.

hdl:10013/epic.29409

Hallikainen, M., Winebrenner, D. P., 1992. The physical basis for sea ice remote sensing, In: Carsey, F. D. (Ed), Microwave Remote Sensing of Sea Ice, Geophysical Monograph, vol. 68, American Geophysical Union, Washington.

Hashin, Z., Shtrikman, S., 1962. A variational approach to the theory of the effective magnetic permeability of multiphase materials, J. Appl. Phys., 33, 3125-3131.

Hobbs, P.V., 1974. Ice physics, Oxford University press, Oxford.

Ingham, M., Pringle, D., Eicken, H., 2008. Cross-borehole resistivity tomography of sea ice, Cold Reg. Sci. Technol., 52, 263-277.

Doi:10.1016/j.coldregions.2007.05.002

Jaccard, C., 1964. Thermodynamics of irreversible processes applied to ice. Physik der Kondensierten Materie, 3, 99-118.

Jones, K. A., Ingham, M., Pringle, D. J., Eicken, H., 2010. Temporal variations in sea ice resistivity: resolving anisotropic microstructure through cross-borehole dc resistivity tomography, J. Geophys. Res., 115, C11023.

Doi:10.1029/2009JC006049

Kawamura, T., 1988. Observations of the internal structure of sea ice by x ray computed tomography, J. Geophys. Res., 93, 2343-2350.

Keller, G. V., Frischknecht, F. C., 1966. International series of monographs in electromagnetic waves Volume 10: Electrical methods in geophysical prospecting, Pergamon Press, Oxford.

Kirkpatrick, S., 1973. Percolation and conduction, Reviews of Modern Physics, 45, 574588.

Kovacs, A., Morey, R., 1978. Radar anisotropy of sea ice due to preferred azimuthal orientation of the horizontal c axes of ice crystals, J. Geophys. Res., 83(C12), 60376046.

Krembs, C., Gradinger, R., Spindler, M., 2000. Implications of brine channel geometry and surface area for the interaction of sympagic organisms in Arctic Sea ice, J. Exp. Mar. Biol. Ecol., 243, 55-80.

Leonard, G. H., Purdie, C. R., Langhorne, P. J., Haskell, T. G., Williams, M. J. M., Frew, R. D., 2006. Observations of platelet ice growth and oceanographic conditions 
during the winter of 2003 in McMurdo Sound Antarctica, J. Geophys. Res., 111, C04012.

Doi: $10.1029 / 2005 J C 002952$

Lepparanta, M., Manninen, T., 1988. The brine and gas content of sea ice with attention to low salinities and high temperatures, Finnish Institute of Marine Research Internal Report, 2, 1-14.

Light, B., Maykut, G. A., Grenfell, T. C., 2003. Effects of temperature on the microstructure of first-year Arctic sea ice, J. Geophys. Res., 108, C2 3051.

Doi: 10.1029/2001JC000887

Lofgren, G., Weeks, W. F., 1969. Effect of growth parameters on substructure spacing in $\mathrm{NaCl}$ ice crystals, J. Glaciol., 8, 153-164.

Loke, M. H., 2004. Tutorial: 2-D and 3-D electrical imaging surveys

Sourced from - http://www.geoelectrical.com/

Loke, M. H., Barker, R. D., 1996a. Rapid least-squares inversion of apparent resistivity pseudosections by a quasi-Newton method, Geophysical prospecting, 44, 131-152.

Loke, M. H., Barker, R. D., 1996b. Practical techniques for 3D resistivity surveys and data inversion, Geophysical prospecting, 44, 499-523.

Looyenga, H., 1965. Dielectric constant of heterogeneous mixtures, Physica, 31, 401406.

Lytle, R. J., Dines, K. A., 1980. Iterative ray tracing between boreholes for underground image reconstruction, IEE transactions on geoscience and remote sensing, GE-18, 234240.

Malmgren, F., 1927. On the properties of sea-ice, Norweg. North Pol. Exped. 'Maud' 1918-1925, Vol. 1, No. 5, 1-67.

Mathews, F. S., Clarke, F. C., 1963. The electrical, structural and topographical characteristics of Arctic sea ice. Volume 1, DECO ELECTRONICS CAMBRIDGE MA Defense Technical Information Center.

Sourced from - http://handle.dtic.mil/100.2/AD408261

McGuinness, M. J., Williams, M. J. M., Langhorne, P. J., Purdie, C., Crook, J., 2009. Frazil deposition under growing sea ice, J. Geophys. Res., 114, C07014.

Doi: 10.1029/2007JC004414.

Mercier, O. R., Hunter, M. W., Callaghan, P. T., 2005. Brine diffusion in first-year sea ice measured by Earth's field PGSE-NMR, Cold Reg. Sci. Technol., 42, 96-105.

Morey, R. M., Kovacs, A., Cox, G. F. N., 1984. Electromagnetic properties of sea ice, Cold Reg. Sci. Technol., 9, 53-75.

Nakawo, M., Sinha, N. K., 1984. A note on brine layer spacing of first-year sea ice, Atmosphere-Ocean, 22, 193-206. 
Notz, D., Worster, M. G., 2008. In situ measurements of the evolution of young sea ice, J. Geophys. Res., 113, C03001.

Doi:10.1029/2007JC004333

Perovich, D. K., 1996. The Optical Properties of Sea Ice, CRREL Monograph, 96-1, $1-25$.

Perovich, D. K., Gow, A. J., 1991. A statistical description of the microstructure of young sea ice, J. Geophys. Res., 96, 16,943-16,953.

Perovich, D. K., Gow, A. J., 1996. A quantitative description of sea ice inclusions, $J$. Geophys. Res., 101, 18,327-18,343.

Petrenko, V. F., 1993. Electrical properties of ice, CRREL Report 93-20, CRREL: Hanover, NH.

Pounder, E. R., 1965. The physics of ice, Pergamon Press, Oxford.

Pringle, D. J., Eicken, H., Trodahl, H. J., Backstrom, L. G. E., 2007. Thermal conductivity of landfast Antarctic and Arctic sea ice, J. Geophys. Res., 112, C04017.

Doi:10.1029/2006JC003641

Pringle, D., Dubuis, G., Eicken, H., 2009a. Impedance measurements of the complex dielectric permittivity of sea ice at $50 \mathrm{MHz}$ : pore microstructure and potential for salinity monitoring, J. Glaciol., 55, 81-94.

Pringle, D. J., Miner, J. E., Eicken, H., Golden, K., 2009b. Pore space percolation in sea ice single crystals, J. Geophys. Res., 114, C12017.

Doi:10.1029/2008JC005145

Sasaki, Y., 1989. Two-dimensional joint inversion of magnetotelluric and dipole-dipole resistivity data, Geophysics, 45, 254-262.

Sasaki, Y., 1992. Resolution of resistivity tomography inferred from numerical simulation, Geophysical Prospecting, 40, 453-463.

Shokr, M. E., Sinha, N. K., 1994. Arctic sea ice microstructure observations relevant to microwave scattering, Arctic, 47, 265-279.

Sihvola, A. H., Kong, J. A., 1988. Effective permittivity of dielectric mixtures, IEEE Trans. on Geoscience and Remote Sensing, 26, 420-429.

Smith, I. J., Langhorne, P. J., Trodahl, H. J., Haskell, T. G., Cole, D. M., 1998. Platelet ice - the McMurdo Sound debate, in Ice in Surface Waters, Proceedings of International Association for Hydraulic Research 14th International Symposium on Ice, Potsdam, New York, July 27-31, 1998, 829-834.

Stogryn, A., 1971. Equations for calculating the dielectric constant of saline water, IEEE Trans. Microwave Theory and Techniques, 19(8), 733-736. 
Stogryn, A., Desargant, G. J., 1985. The dielectric properties of brine in sea ice at microwave frequencies, IEEE Trans. on Antennas and Propagation, AP-33, 523-532.

Telford, W. M., Geldart, L. P., Sheriff, R. E., Keys, D. A., 1977. Applied Geophysics, Cambridge University Press, New York.

Thyssen, F., Kohnen, H., Cowan, M. V., Timco, G. W., 1974. DC Resistivity measurements on sea ice near Pond Inlet, N.W.T (Baffin Island), POLARFORSCHUNG, 44, 117-126.

Timco, G. W., 1979. An analysis of the in-situ resistivity of sea ice in terms of its microstructure, J. Glaciol., 22, 461-471.

Timco, G. W., Frederking, R. M. W., 1996. A review of sea ice density, Cold Reg. Sci. Technol., 24, 1-6.

Timco, G. W., Johnston, M. E., 2002. Sea ice strength during the melt season, Ice in the environment: Proceedings of the $16^{\text {th }}$ IAHR International Symposium on Ice, Dunedin, New Zealand, 2 - 6 December, 2002.

Tinga, W. R., Voss, W. A. G., Blossey, D. F., 1973. Generalised approach to multiphase dielectric mixture theory, J. Appl. Phys., 44, 3897-3092.

Toyama, Y., Nishio, F., Hasegawa, J., 2001. Characteristics of DC conductivity measurement in sea ice, Seppyo, 63, 253-264.

Trodahl, H. J., McGuinness, M. J., Langhorne, P. J., Collins, K., Pantoja, A. E., Smith, I. J., Haskell, T. G., 2000. Heat transport in McMurdo Sound first-year fast ice, J. Geophys. Res., 105, 11347-11358.

Vant, M. R., Ramseier, R. O., Makios, V., 1978. The complex-dielectric constant of sea ice at frequencies in the range 0.1-40 GHz, J. Appl. Phys., 49, 1264-1280.

Weeks, W. F., Ackley, S. F., 1986. The growth structure and properties of sea ice, In: Untersteiner, N. (Ed.), The geophysics of sea ice, Plenum Press, New York, pp9-164.

Wolff, E. W., Miners, W. D., Moore, J. C., Paren, J. G., 1997. Factors Controlling the Electrical Conductivity of Ice from the Polar Regions-A Summary, J. Phys. Chem. B, 101, 6090-6094. 